Supporting Information

\title{
Controlled Cyclopolymerization of 1,5-Hexadiynes to Give Narrow Band Gap Conjugated Polyacetylenes Containing Highly Strained Cyclobutenes
}

\author{
Cheol Kang, Kijung Jung, Sojeong Ahn, and Tae-Lim Choi* \\ Department of Chemistry, Seoul National University, Seoul, 08826, Republic of Korea \\ * e-mail: tlc@snu.ac.kr
}




\section{Table of Contents}

1. Supporting Figures

$\mathrm{S} 3-\mathrm{S} 15$

2. General Experimental. S16

3. Experimental Procedures for Preparation of Monomers $\mathrm{S} 17-\mathrm{S} 20$

4. General Procedure for Polymerization. S20

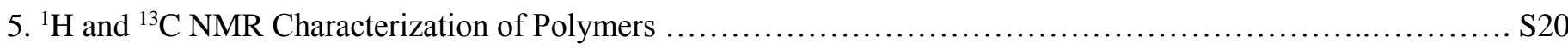

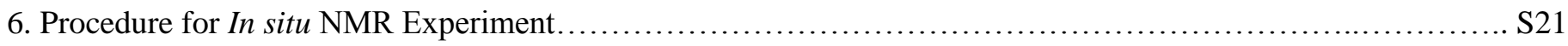

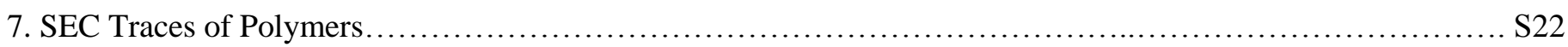

8. ${ }^{1} \mathrm{H}$ and ${ }^{13} \mathrm{C}$ NMR Spectra of New Compounds ................................................ S23 - S41

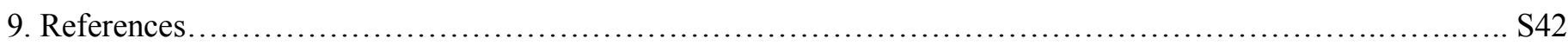

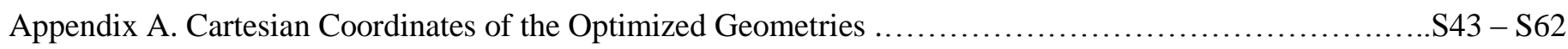

Appendix B. Vibrational Frequencies $\left(\mathrm{in}_{\mathrm{cm}}{ }^{-1}\right.$ ) of the Optimized Structures ...............................S63 - S82 


\section{Supporting Figures}

Table S1. Polymerization of simple 1,5-hexadiyne and tetra-substituted benzyl-containing monomers with different stereochemistry

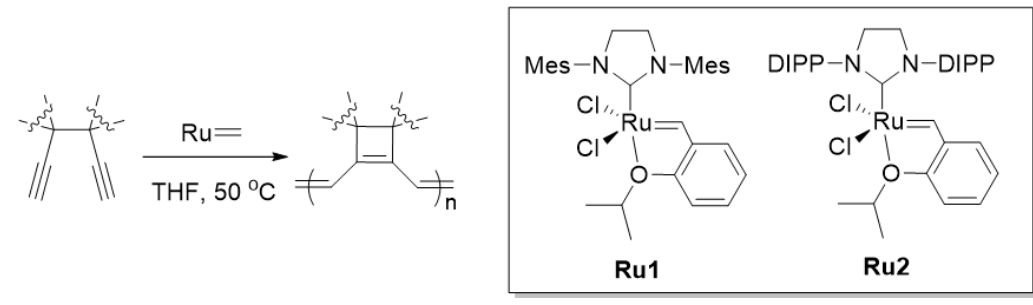

\begin{tabular}{|c|c|c|c|c|c|c|}
\hline monomer & cat & $\mathbf{M} / \mathbf{I}$ & conc & time & $\operatorname{conv}^{a}$ & yield $^{b}$ \\
\hline 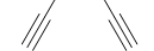 & Ru1 & 20 & $0.5 \mathrm{M}$ & 3 & $10 \%$ & - \\
\hline $\mathrm{BnO} \quad \mathrm{OBn}$ & Ru1 & 20 & $0.5 \mathrm{M}$ & 24 & $0 \%$ & - \\
\hline$\left.\right|^{\mathrm{HO}}$ & Ru2 & 30 & $0.5 \mathrm{M}$ & 3 & $54 \%$ & - \\
\hline & Ru2 & 30 & $1 \mathrm{M}$ & 3 & $69 \%$ & $35 \%$ \\
\hline$\frac{\mathrm{HO}}{\|_{\|}^{\mathrm{OBn}}}$ & Ru2 & 30 & $0.5 \mathrm{M}$ & 3 & $0 \%$ & - \\
\hline
\end{tabular}

${ }^{a}$ Calculated from crude ${ }^{1} \mathrm{H}$ NMR. ${ }^{b}$ Isolated yield. 
${\sqrt{\left.\right|^{-O T I P S}}}^{\mathrm{C}_{11} \mathrm{H}_{23}}$

M8

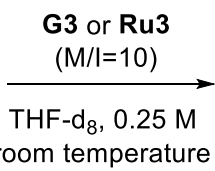

room temperature

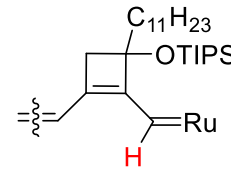

In situ NMR observation

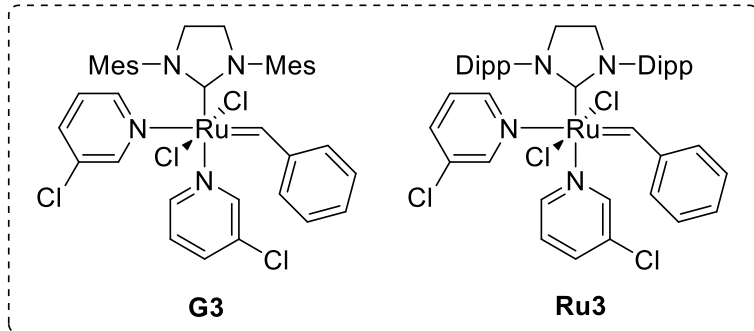

(a) $\mathbf{G} 3$

(b) Ru3

G3
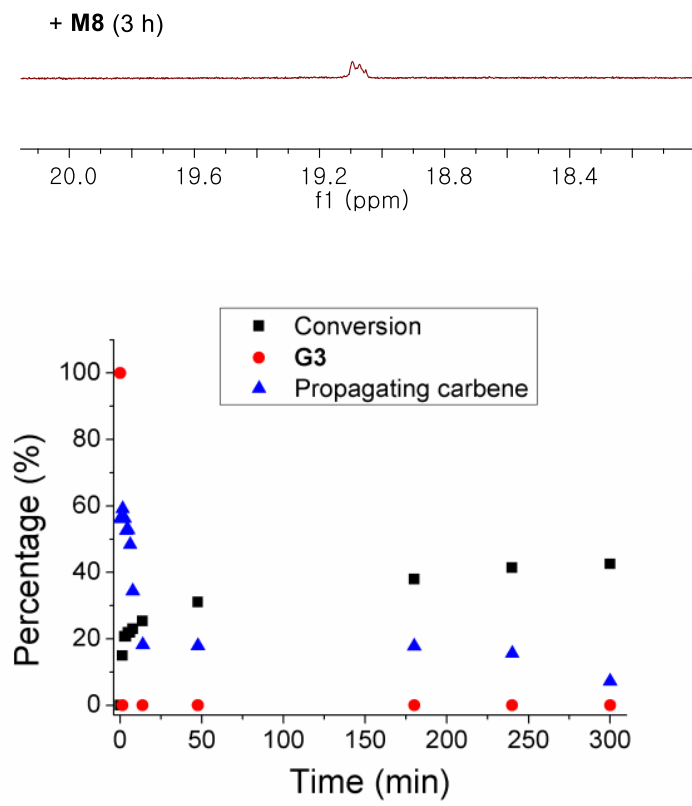

Ru3

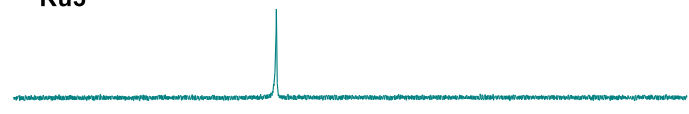

$+M 8(18 h)$

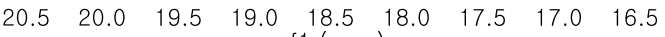

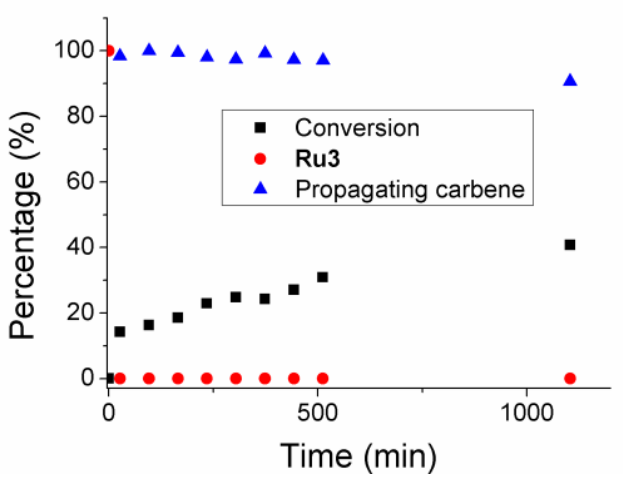

Figure S1. Carbene, conversion, and propagating carbene changes for the polymerization of M8 (M/I=10) using (a) G3 or (b) Ru3. The reaction was conducted in THF- $d_{8}\left(0.25 \mathrm{M}\right.$ for monomer) at room temperature and monitored by in situ ${ }^{1} \mathrm{H}$ NMR analysis. The propagating carbene from $\mathbf{G 3}$ decomposed severely during polymerization, while the propagating carbene from Ru3 maintained high throughout the reaction. 
$\sqrt{\mathrm{C}_{11} \mathrm{H}_{23}}$

M8
Ru2 or Ru3 $(\mathrm{M} / \mathrm{l}=10)$

THF- $\mathrm{d}_{8}, 0.25 \mathrm{M}$

$50{ }^{\circ} \mathrm{C}$

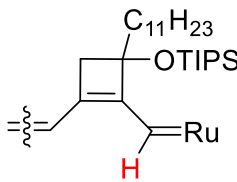

In situ NMR observation

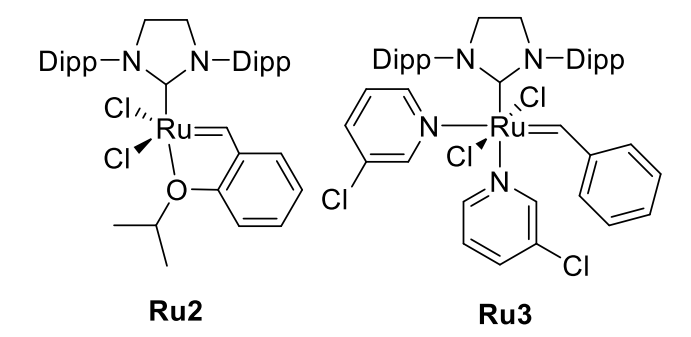

(a) Ru2

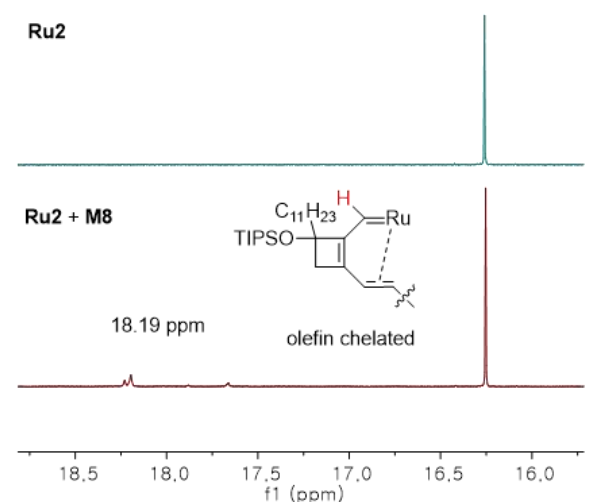

(b) Ru3

Ru3

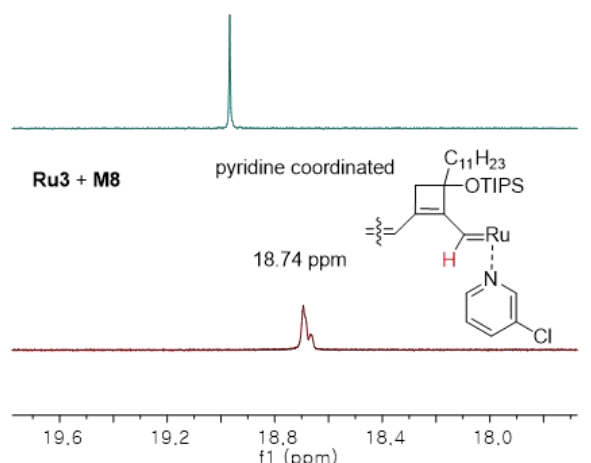

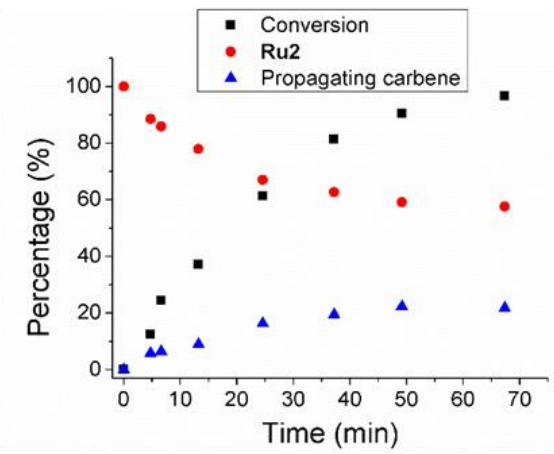

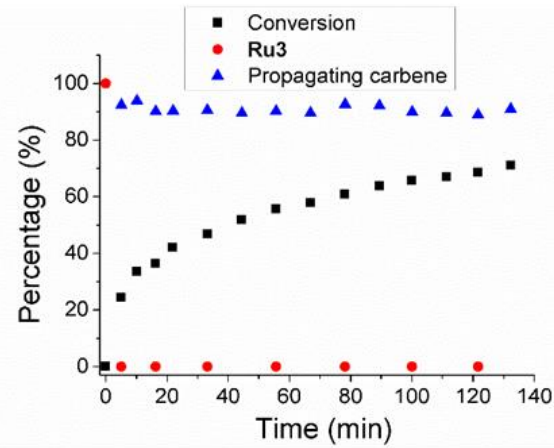

\begin{tabular}{cccc}
\hline & $\boldsymbol{k}_{\mathrm{i}}\left(\min ^{-1} \mathrm{M}^{-1}\right)$ & $\boldsymbol{k}_{\mathrm{p}}\left(\min ^{-1} \mathrm{M}^{-1}\right)$ & $\boldsymbol{k}_{\mathrm{i}} / \boldsymbol{k}_{\mathrm{p}}$ \\
\hline (a) & 0.056 & 2.04 & 0.027 \\
(b) & $>1.18$ & 0.27 & $>4.4$ \\
\hline
\end{tabular}

Figure S2. Carbene, conversion, and propagating carbene changes for the polymerization of M8 (M/I = 10) using (a) Ru2 or (b) Ru3. The reaction was conducted in THF- $d_{8}\left(0.25 \mathrm{M}\right.$ for monomer) at $50^{\circ} \mathrm{C}$ and monitored by in situ ${ }^{1} \mathrm{H}$ NMR analysis. The propagating carbene from $\mathbf{R u} 2$ was shown at $18.19 \mathrm{ppm}$ which is putatively assigned as olefin chelated complex, while that from Ru3 (pyridine bound complex) was shown at more downfield region of $18.74 \mathrm{ppm}$. All of the $k_{\mathrm{i}}$ and $k_{\mathrm{p}}$ values were calculated based on the equations; $-\mathrm{d}[\mathrm{I}] / \mathrm{dt}=k_{\mathrm{i}}[\mathrm{M}][\mathrm{I}]$ and $-\mathrm{d}[\mathrm{M}] / \mathrm{dt}=k_{\mathrm{p}}[\mathrm{M}][\mathrm{I}]$. The exact $k_{\mathrm{i}}$ from Ru3 could not be obtained due to too fast initiation. 
Detailed Characterization of the Polymer Structures $\left({ }^{1} \mathrm{H},{ }^{13} \mathrm{C}\right.$, HSQC, and HMBC NMR analyses)
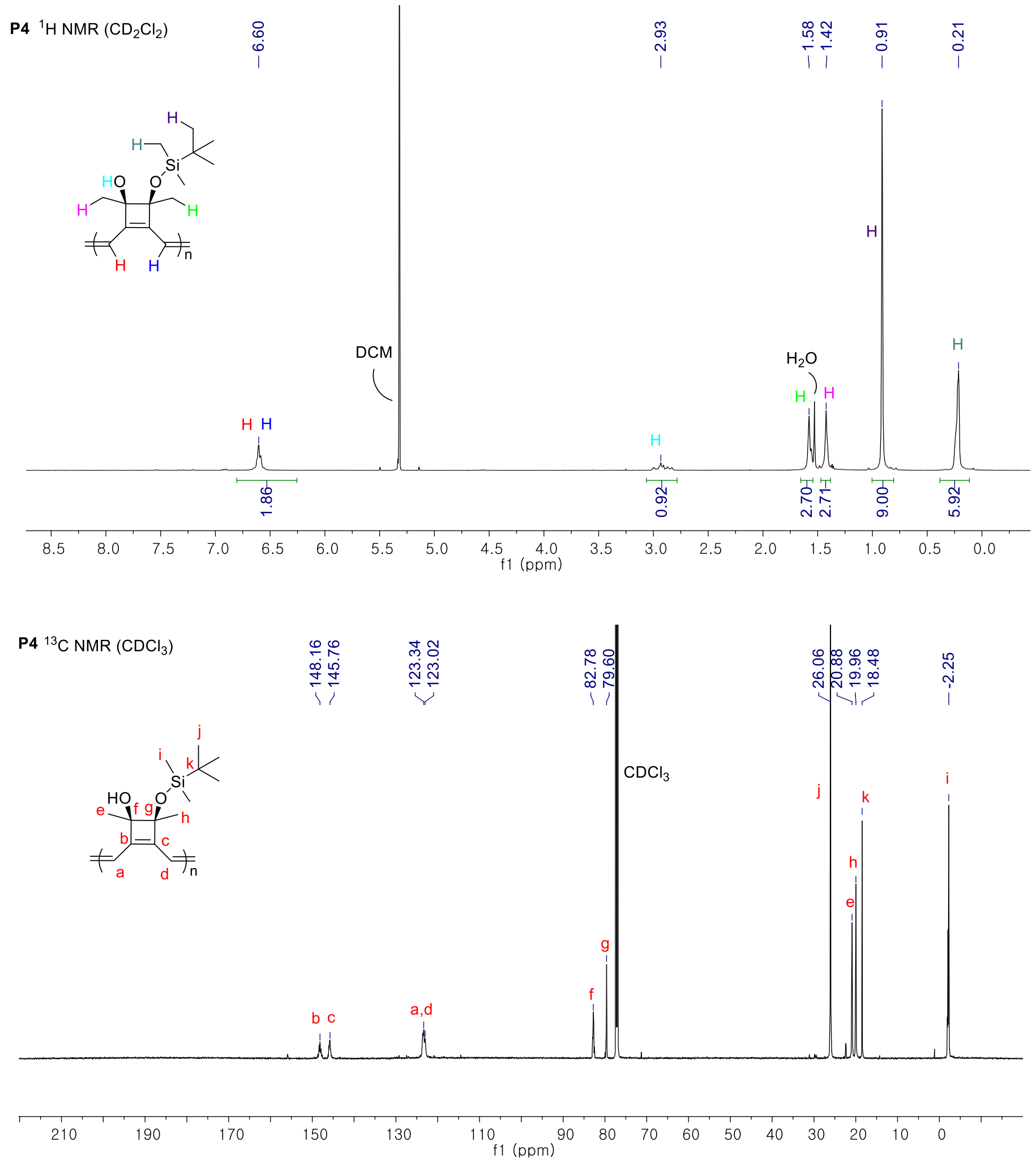

Figure S3. ${ }^{1} \mathrm{H}$ (up) and ${ }^{13} \mathrm{C}$ (down) NMR spectra of $\mathbf{P 4}$. 

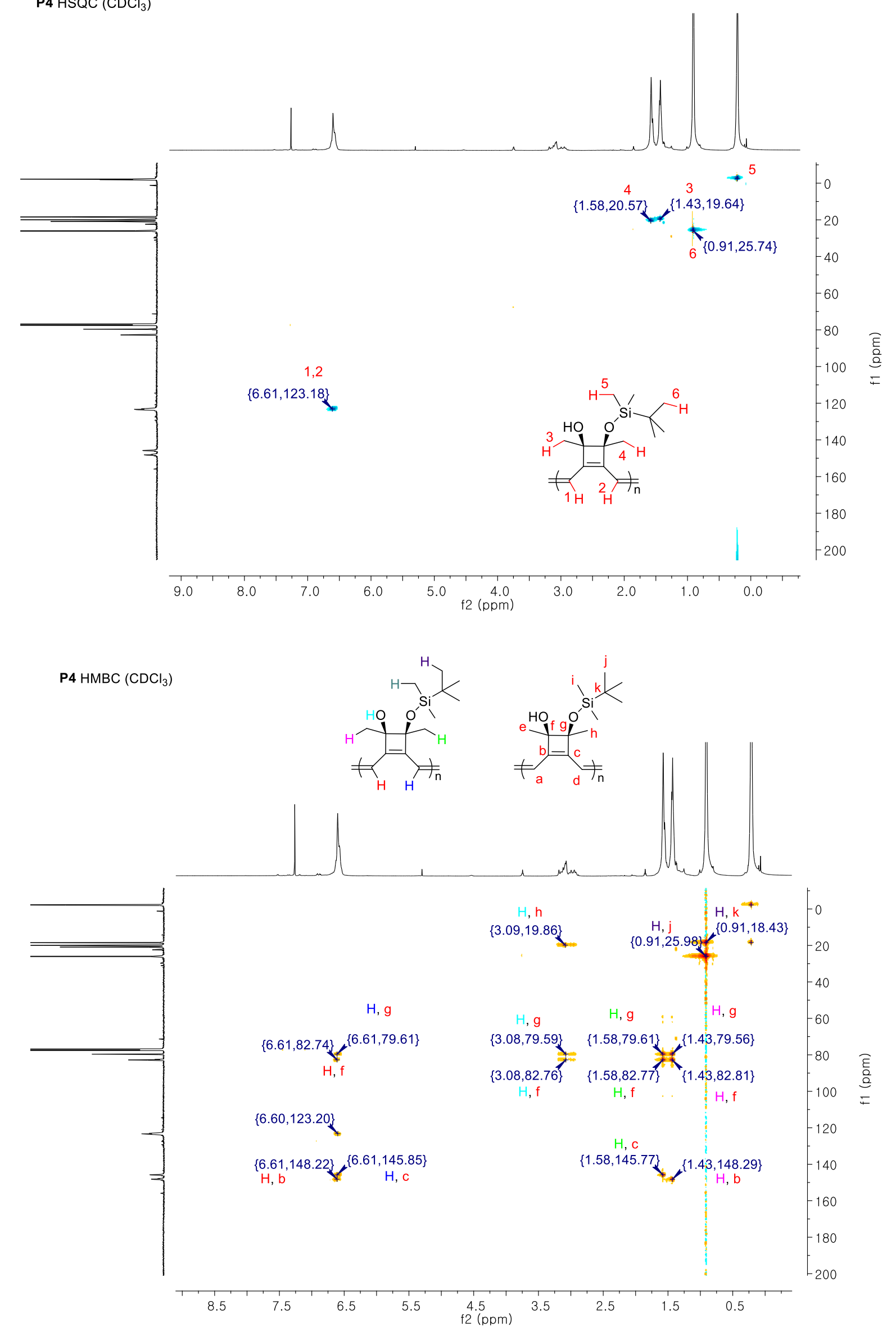

Figure S4. ${ }^{1} \mathrm{H}-{ }^{13} \mathrm{C}$ HSQC (Heteronuclear Single Quantum Coherence) NMR (up) and ${ }^{1} \mathrm{H}-{ }^{13} \mathrm{C}$ HMBC (Heteronuclear Multiple Bond Correlation) NMR (down) spectra of $\mathbf{P 4}$. 
P6 ${ }^{1} \mathrm{H}$ NMR $\left(\mathrm{CD}_{2} \mathrm{Cl}_{2}\right) \quad$ f.<smiles>C=CC1=C(C=CC)[C@@]2(C)O[C@@H](c3ccccc3)O[C@@]12C</smiles>

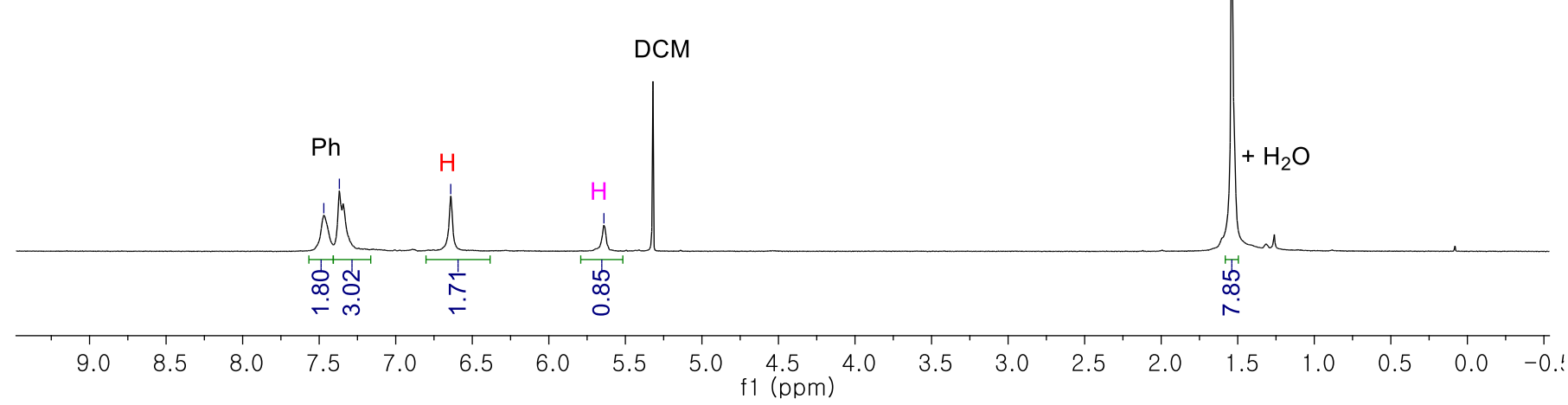

P6 ${ }^{13} \mathrm{C}$ NMR $\left(\mathrm{CD}_{2} \mathrm{Cl}_{2}\right)$

เิ่

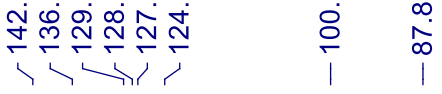
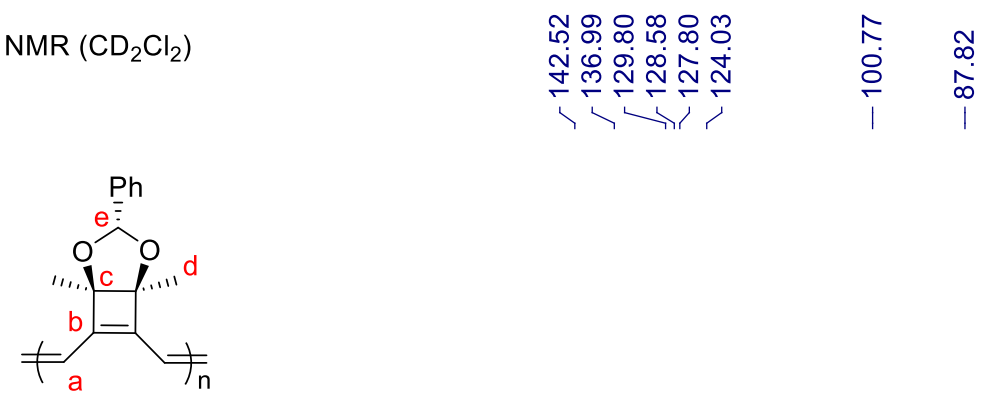

ஸั่
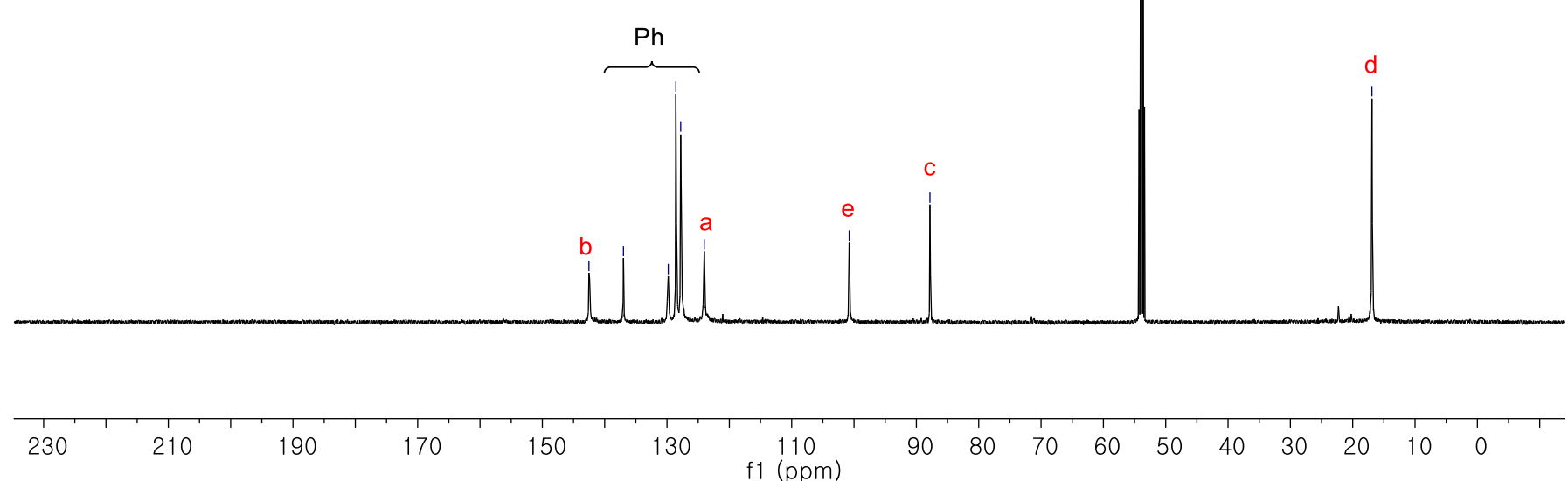

Figure S5. ${ }^{1} \mathrm{H}$ (up) and ${ }^{13} \mathrm{C}$ (down) NMR spectra of P6. 
P6 HSQC $\left(\mathrm{CD}_{2} \mathrm{Cl}_{2}\right)$

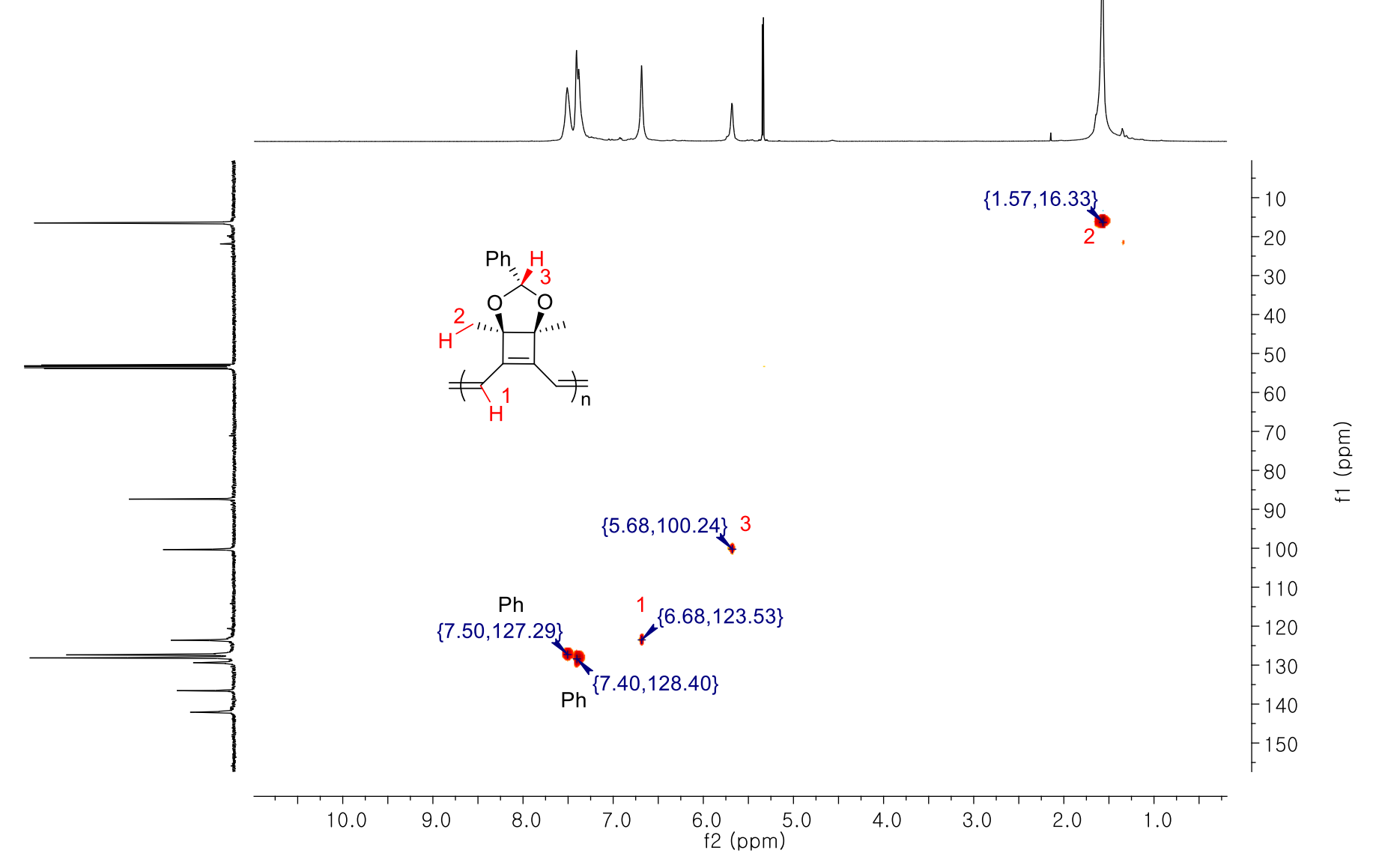

Figure S6. ${ }^{1} \mathrm{H}-{ }^{13} \mathrm{C}$ HSQC NMR spectrum of $\mathbf{P 6}$. 
P8 ${ }^{1} \mathrm{H}$ NMR $\left(\mathrm{CD}_{2} \mathrm{Cl}_{2}\right)$
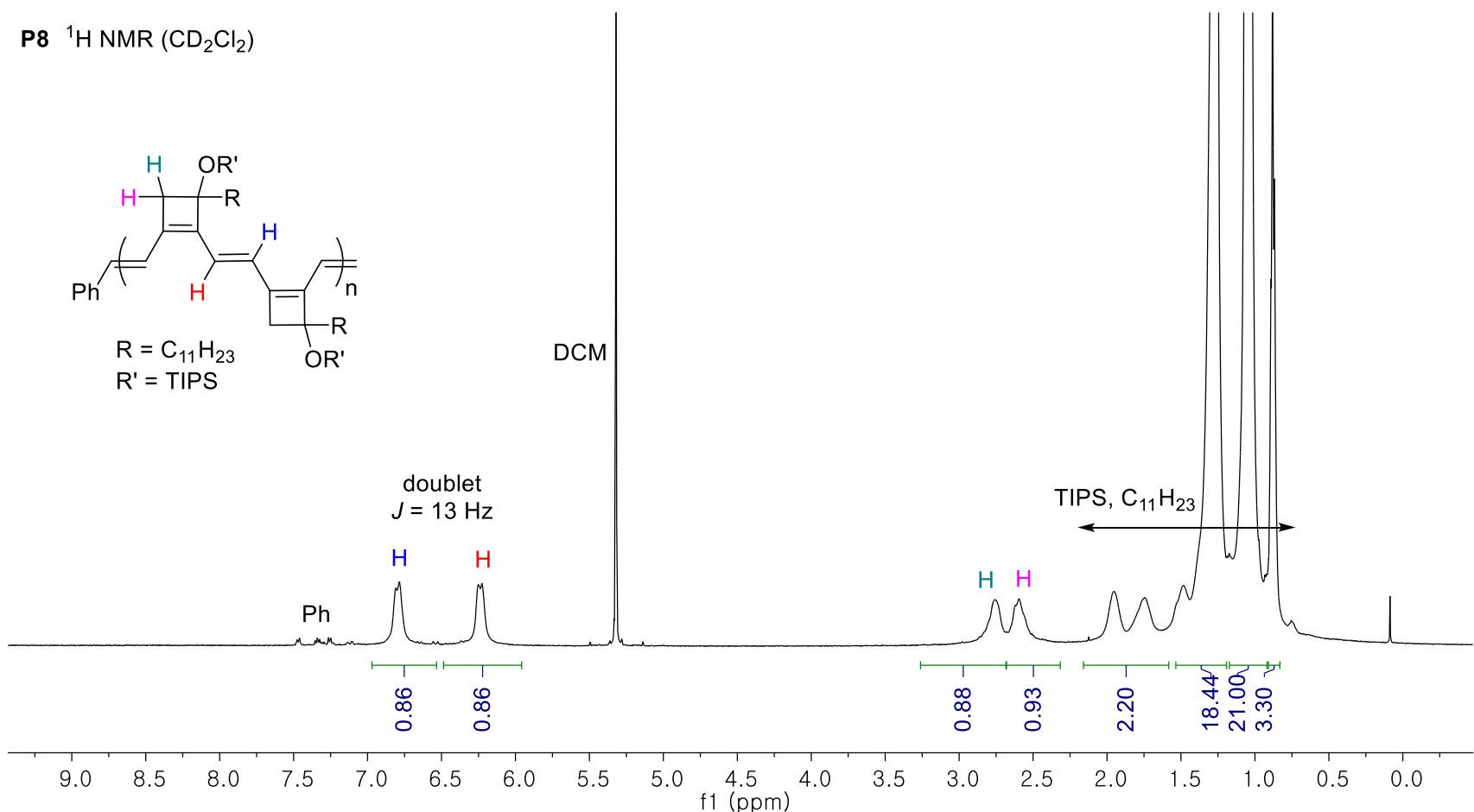

P8 ${ }^{13} \mathrm{C}$ NMR $\left(\mathrm{CD}_{2} \mathrm{Cl}_{2}\right)$

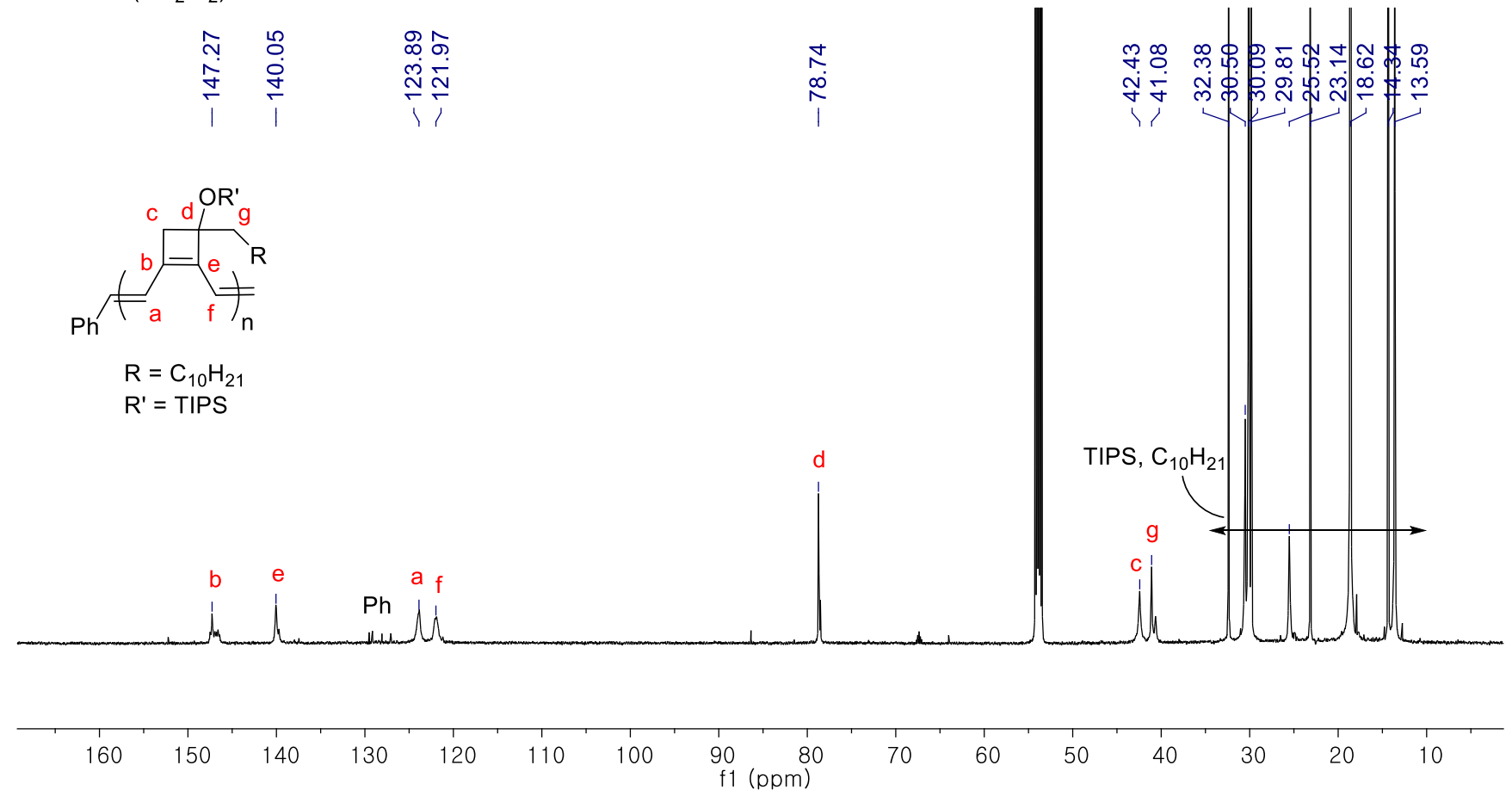

Figure S7. ${ }^{1} \mathrm{H}$ (up) and ${ }^{13} \mathrm{C}$ (down) NMR spectra of $\mathbf{P 8}$. 
P8 HSQC $\left(\mathrm{CD}_{2} \mathrm{Cl}_{2}\right)$

$\mathrm{R}=\mathrm{C}_{10} \mathrm{H}_{21}$

$\mathrm{R}^{\prime}=$ TIPS
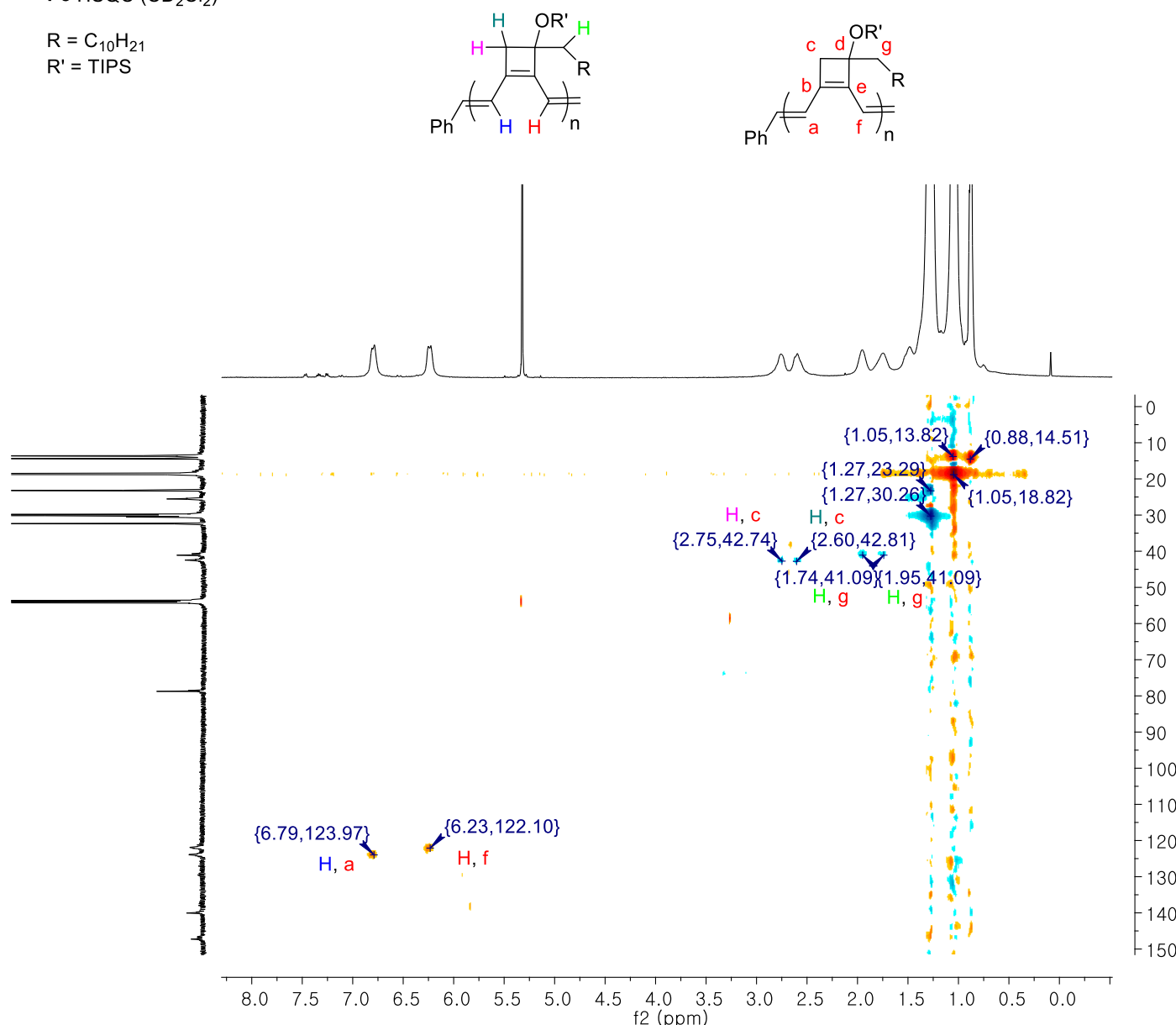

P8 $\mathrm{HMBC}\left(\mathrm{CD}_{2} \mathrm{Cl}_{2}\right)$

$\mathrm{R}=\mathrm{C}_{10} \mathrm{H}_{21}$
$\mathrm{R}^{\prime}=$ TIPS
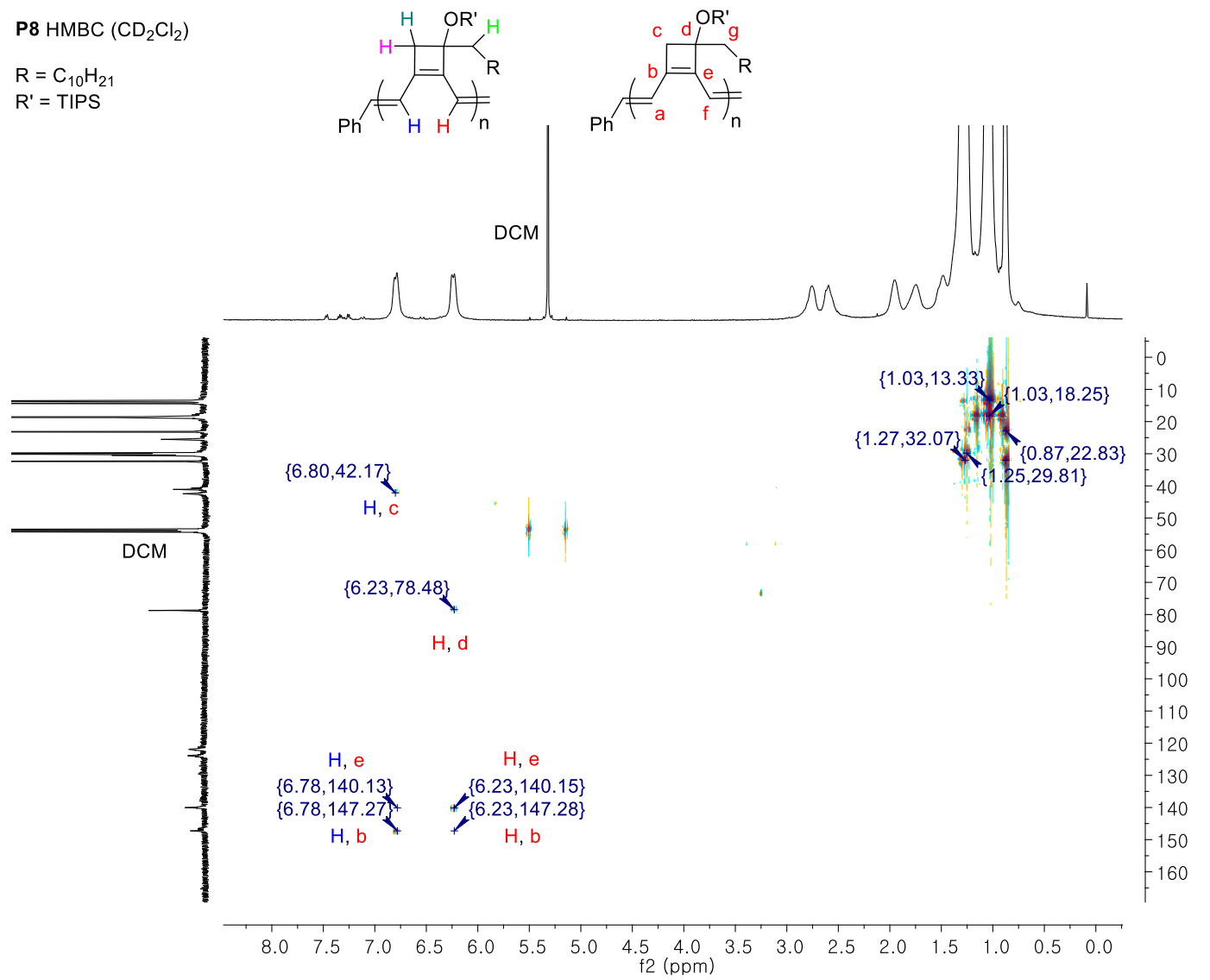

Figure S8. ${ }^{1} \mathrm{H}-{ }^{13} \mathrm{C}$ HSQC NMR (up) and ${ }^{1} \mathrm{H}-{ }^{13} \mathrm{C}$ HMBC NMR (down) spectra of $\mathbf{P 8}$. 


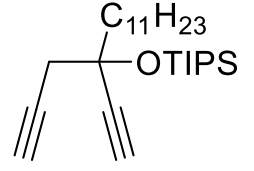

M8

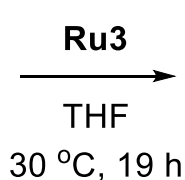

$30{ }^{\circ} \mathrm{C}, 19 \mathrm{~h}$

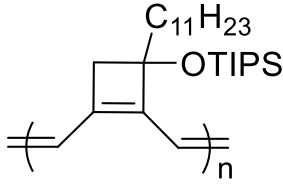

P8 conv. $=56 \%$

$M_{\mathrm{n}}=6.8 \mathrm{kDa}$

$\oplus=1.37$

Figure S9. Polymerization of M8 using Ru3 at $30{ }^{\circ} \mathrm{C}$. The conversion was as low as $56 \%$ even after $19 \mathrm{~h}$, giving an $M_{\mathrm{n}}$ of $6.8 \mathrm{kDa}$ and a relatively broad dispersity of 1.37 .
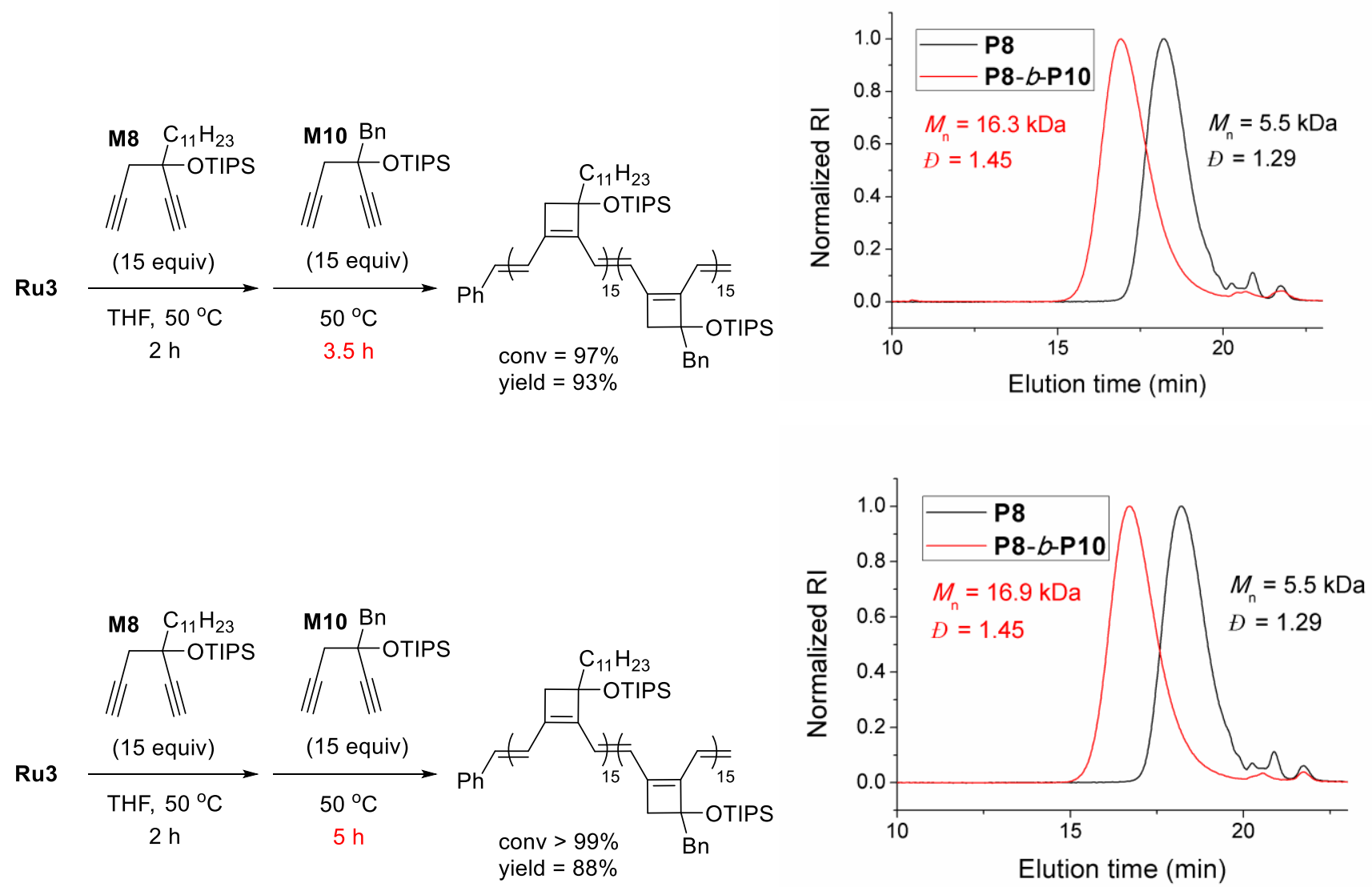

Figure S10. Block copolymerization by sequential addition of M8 and M10. The longer reaction time for the second block propagation ( 3.5 vs $5 \mathrm{~h}$ ) did not broaden the dispersity of the resulting polymer, implying that the chain transfer reaction did not occur under the reaction condition. 
Table S2. Optical and Physical Properties of P3 - P10

\begin{tabular}{|c|c|c|c|c|c|c|}
\hline \multirow{2}{*}{} & \multicolumn{2}{|c|}{ Solution } & \multicolumn{2}{c|}{ Film } & \multirow{2}{*}{$E_{\text {HOMO }}(\mathrm{eV})$} & $T_{\mathrm{d}}\left({ }^{\circ} \mathrm{C}\right)$ \\
\cline { 2 - 5 } & $\lambda_{\text {abs }}(\mathrm{nm})$ & $E_{\mathrm{g}}{ }^{\mathrm{opt}}(\mathrm{eV})$ & $\lambda_{\text {abs }}(\mathrm{nm})$ & $E_{\mathrm{g}}{ }^{\mathrm{opt}}(\mathrm{eV})$ & & \\
\hline $\mathbf{P 3}$ & 552,595 & 1.96 & 544 & 1.94 & -5.12 & 177 \\
\hline $\mathbf{P 4}$ & 550,592 & 1.97 & 538 & 1.92 & -5.17 & 175 \\
\hline $\mathbf{P 6}$ & 556,600 & 1.95 & 551 & 1.90 & -5.23 & 284 \\
\hline $\mathbf{P 8}$ & 582 & 1.81 & 569 & 1.77 & -5.26 & 328 \\
\hline P9 & 597,646 & 1.80 & 602,652 & 1.74 & -5.34 & 348 \\
\hline P10 & 603,652 & 1.77 & 598 & 1.75 & -5.15 & 334 \\
\hline
\end{tabular}
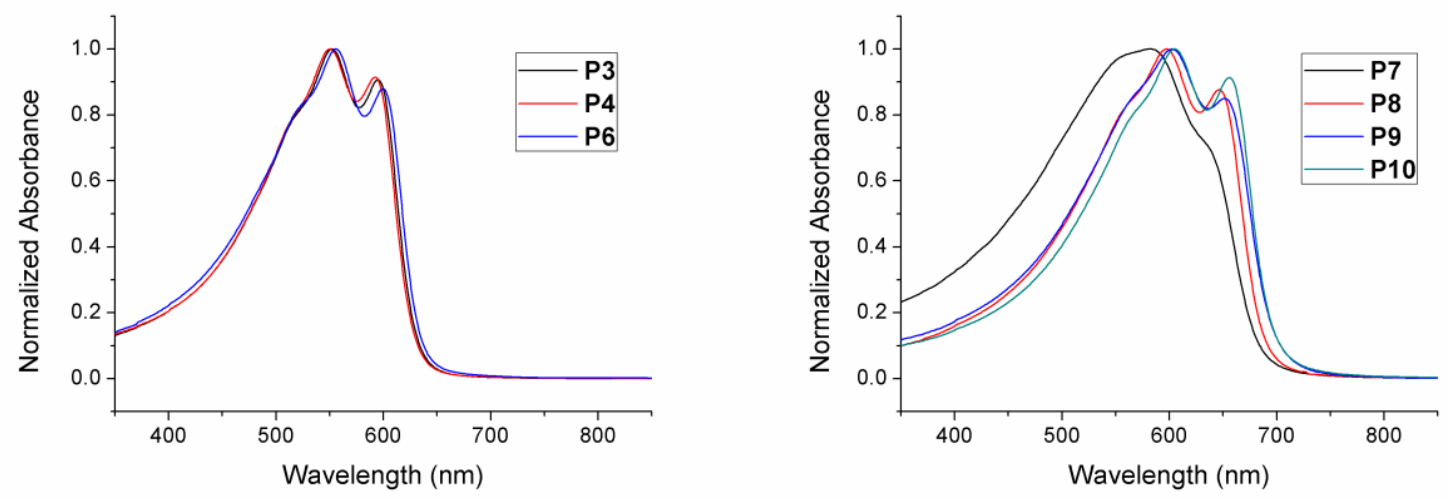

Figure S11. UV-Vis spectra of P3 - P10 in THF solution (ca. $0.01 \mathrm{~g} / \mathrm{L}$ ).
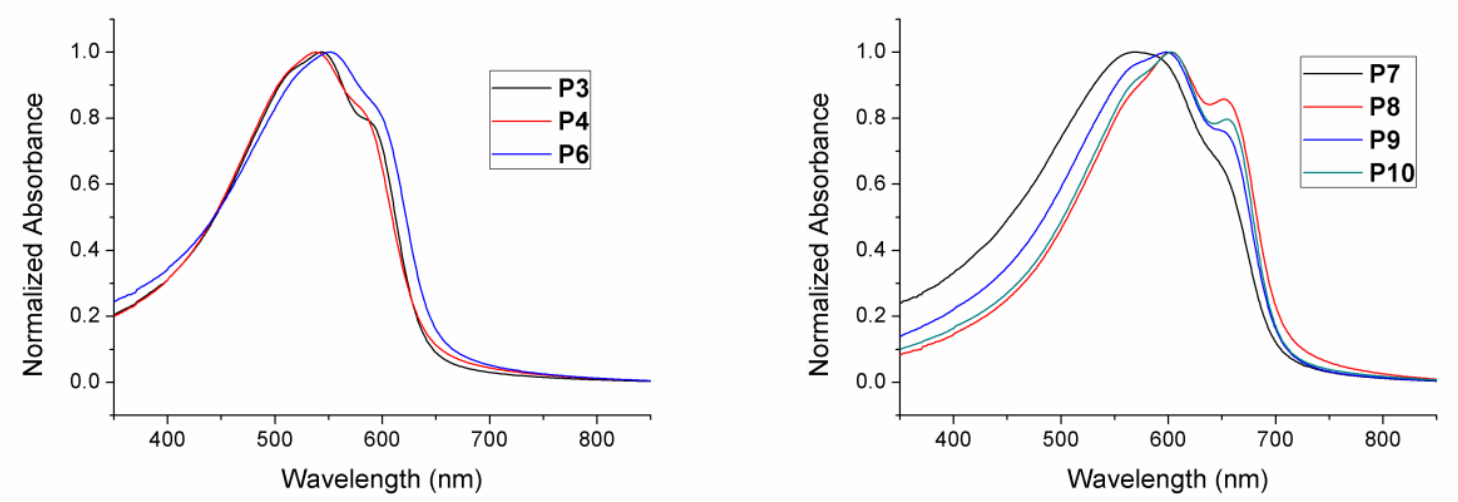

Figure S12. UV-Vis spectra of P3 - P10 in film state. 

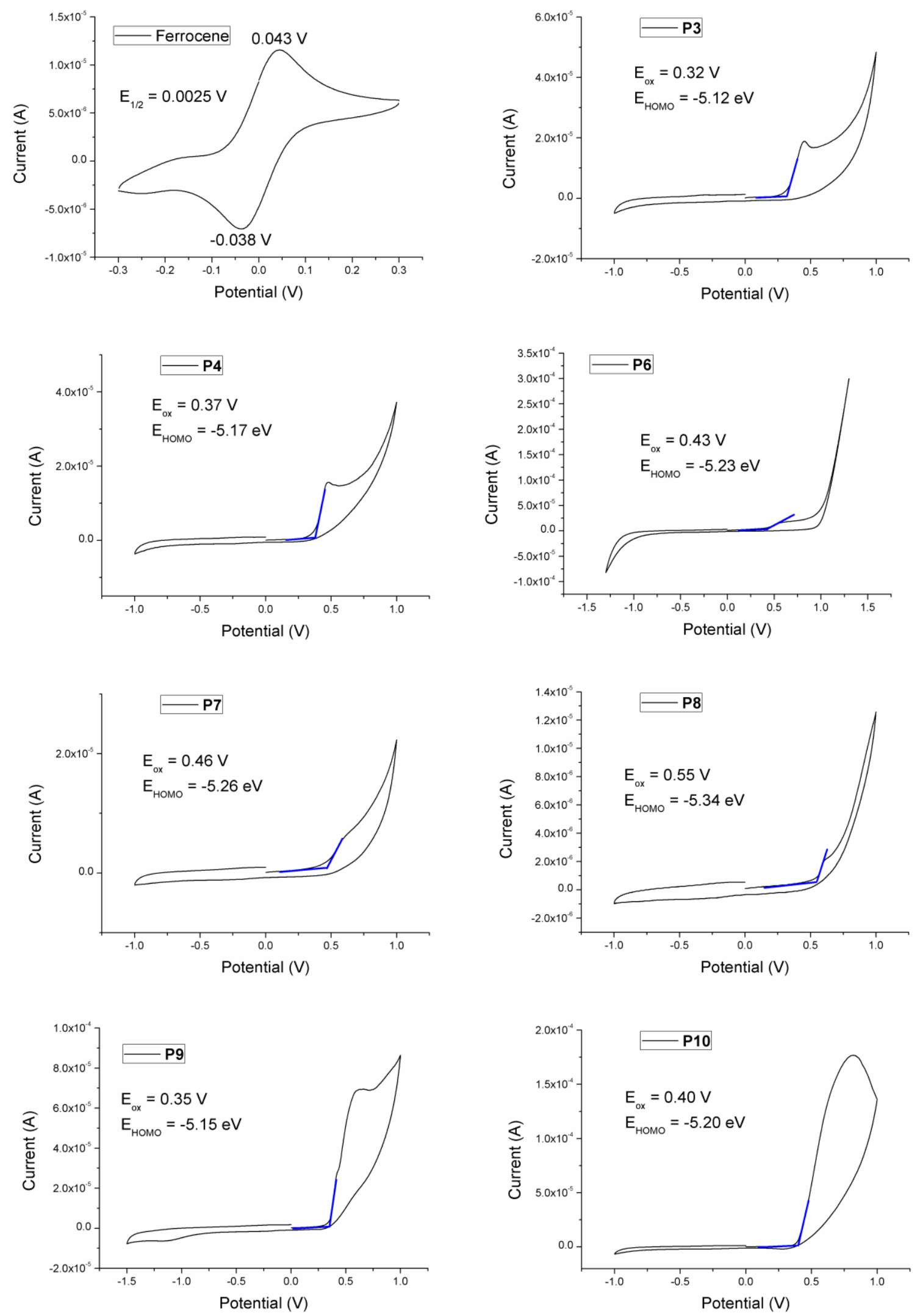

Figure S13. Cyclic voltammograms of ferrocene (measured in DMSO) and P3 - P10. Polymer samples were prepared as film deposited on a working electrode, using a reference electrode of $\mathrm{Ag} / \mathrm{Ag}^{+}\left(0.1 \mathrm{M} \mathrm{AgNO}_{3}\right.$ in acetonitrile) with a platinum wire counter electrode. All the polymers were measured using $\mathrm{NBu}_{4} \mathrm{PF}_{6}$ electrolyte in DMSO solution $(0.1 \mathrm{M})$. The absolute energy level was obtained using ferrocene/ferrocenium as an internal standard. The oxidation potential of ferrocene was regarded as $-4.8 \mathrm{eV}$. 

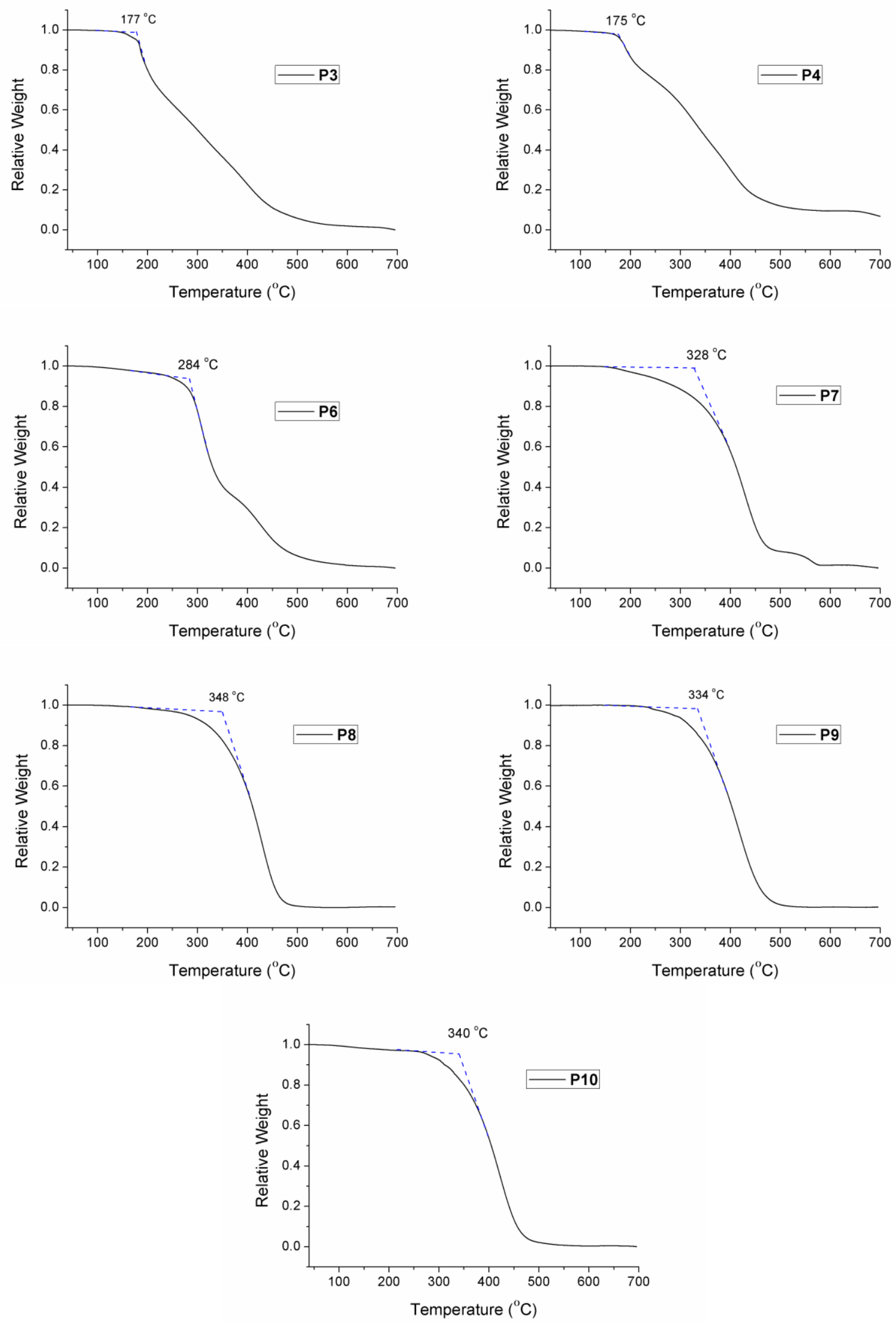

Figure S14. TGA curves of $\mathbf{P 3}$ - P10. The samples were equilibrated at $30^{\circ} \mathrm{C}$, then heated at $10{ }^{\circ} \mathrm{C} / \mathrm{min}$ to $700{ }^{\circ} \mathrm{C}$. The onset points were reported as the thermal decomposition temperatures $\left(T_{\mathrm{d}}\right)$. 


\section{General Experimental}

\section{Characterization}

${ }^{1} \mathrm{H}$ NMR and ${ }^{13} \mathrm{C}$ NMR were recorded by Varian/Oxford As-500 (500 MHz for ${ }^{1} \mathrm{H}, 125 \mathrm{MHz}$ for $\left.{ }^{13} \mathrm{C}\right)$, Agilent 400-MR (400 MHz for ${ }^{1} \mathrm{H}$ and $100 \mathrm{MHz}$ for $\left.{ }^{13} \mathrm{C}\right)$, and Bruker AVANCE $600\left(600 \mathrm{MHz}\right.$ for ${ }^{1} \mathrm{H}$ and $150 \mathrm{MHz}$ for $\left.{ }^{13} \mathrm{C}\right)$. In situ NMR experiments at $50{ }^{\circ} \mathrm{C}$ were conducted by using Bruker AVANCE III 500 (500 MHz for $\left.{ }^{1} \mathrm{H}\right)$. Size exclusion chromatography (SEC) analyses were carried out with Waters system (1515 pump and 2707 autosampler) and Shodex GPC LF-804 column eluted with THF (HPLC grade, TEDIA) and filtered through a $0.2 \mu \mathrm{m}$ PTFE filter (Whatman ${ }^{\circledR}$ ) before injection. The flow rate was $1.0 \mathrm{~mL} / \mathrm{min}$ and temperature of the column was maintained at $35^{\circ} \mathrm{C}$. Wyatt OptiLab T-rEx refractive index detector was used for molecular weight measurement. High-resolution mass spectroscopy (HRMS) analyses were performed by ultra high resolution ESI Q-TOF mass spectrometer (Bruker) and FAB mass spectrometer JMS-700(JEOL). Elemental analysis was carried out by Flash2000 (Thermo Fisher Scientific). UV/Vis spectra were obtained by UV-vis Spectrometer V-650 (Jasco Inc.). Cyclic voltammetry (CV) measurements were carried out by CHI 660 Electrochemical Analyzer (CH Instruments, Insc.) Thermogravimetric analysis (TGA) was carried out under $\mathrm{N}_{2}$ gas at a scan rate of $10^{\circ} \mathrm{C} / \mathrm{min}$ with Q50 model device (TA Instruments).

\section{Materials}

All reagents which are commercially available from Sigma-Aldrich ${ }^{\circledR}$, Tokyo Chemical Industry Co. Ltd., Acros Organics, Alfa Aesar $^{\circledR}$, and Umicore (Ru2) were used without further purification. Ru3 was prepared from dichloro[1,3-bis(2,6-isopropylphenyl)2-imidazolidinylidene](benzylidene)(tricyclohexylphosphine)ruthenium(II) purchased from BOC Sciences, by following the modified procedure from the literature method (details below). ${ }^{1}$ Tetrahydrofuran for the polymerization were purified by distillation and degassed further by Ar bubbling for 10 minutes before performing reactions. THF- $d_{8}(99.50 \% \mathrm{D}, 0.75 \mathrm{~mL})$ was purchased from Deutero $\mathrm{GmbH}$ and used without further purification.

\section{Computational Method}

All the theoretical calculations were performed with the Gaussian 09 program. ${ }^{2}$ The geometries of the singlet ground states of model oligomer structures were optimized by the density functional theory (DFT) method with B3LYP hybrid function. The 6-31G(d) basis sets were employed for all atoms. Vibrational frequencies were calculated with the optimized geometries at the same level of theory optimizations. All the structures were confirmed by observing no or only one imaginary frequency. 


\section{Experimental procedures for the preparation of monomers and Ru3}

Compounds M1, ${ }^{3} 1,{ }^{3} \mathbf{2},{ }^{4}$ and $3^{5}$ were prepared by literature methods. Schlenk line techniques were used for all the synthesis using Ar gas for the inert condition.

\section{Synthesis of M2}

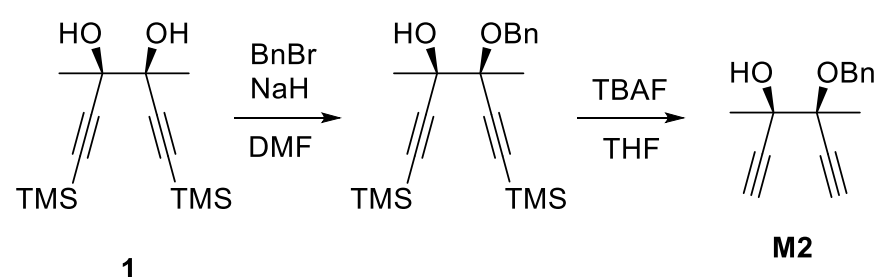

Compound 1 (139 mg, $1.01 \mathrm{mmol})$ was dissolved in DMF (3 ml), and $\mathrm{NaH}$ (60\% dispersion in mineral oil, $44 \mathrm{mg}, 1.1 \mathrm{mmol})$ was slowly added to the solution at $0{ }^{\circ} \mathrm{C}$. Then, benzyl bromide $(0.13 \mathrm{ml}, 1.1 \mathrm{mmol})$ was added and the reaction mixture was stirred at room temperature for $2.5 \mathrm{~h}$. After quenching with a saturated $\mathrm{NH}_{4} \mathrm{Cl}$ aqueous solution, the organic layer was washed with brine and extracted by ethyl acetate, dried with $\mathrm{MgSO}_{4}$, and concentrated. Purification with flash column chromatography afforded a mixture of the desired product and its TMS-deprotected derivatives. This mixture was dissolved in THF (3 ml), then tetrabutylammonium fluoride solution (1.0 M in THF, $0.77 \mathrm{ml}, 0.77 \mathrm{mmol}$ ) was added. After stirring for $40 \mathrm{~min}$, the reaction mixture was quenched with $\mathrm{NH}_{4} \mathrm{Cl}$ aqueous solution. The organic layer was washed with water and extracted by ethyl acetate, dried with $\mathrm{MgSO}_{4}$, and concentrated. The product was purified by flash column chromatography on silica gel to afford M2 as white powder (46 mg, 0.20 mmol, 20\% via two steps). ${ }^{1} \mathrm{H}$ NMR $\left(400 \mathrm{MHz}, \mathrm{CDCl}_{3}\right) \delta 7.42-7.27(\mathrm{~m}, 5 \mathrm{H}), 4.89(\mathrm{~d}, J=11.3 \mathrm{~Hz}, 1 \mathrm{H}), 4.66(\mathrm{~d}, J=11.3 \mathrm{~Hz}, 1 \mathrm{H})$, $2.85(\mathrm{~s}, 1 \mathrm{H}), 2.65(\mathrm{~s}, 1 \mathrm{H}), 2.50(\mathrm{~s}, 1 \mathrm{H}), 1.63(\mathrm{~s}, 3 \mathrm{H}), 1.60(\mathrm{~s}, 3 \mathrm{H}) .{ }^{13} \mathrm{C} \mathrm{NMR}\left(125 \mathrm{MHz}, \mathrm{CDCl}_{3}\right) \delta$ 138.69, 128.44, 127.63, 127.56, 85.76, 82.34, 79.11, 76.41, 73.61, 72.60, 67.46, 24.48, 21.18. HRMS (FAB): m/z for $\mathrm{C}_{15} \mathrm{H}_{17} \mathrm{O}_{2}[\mathrm{M}+\mathrm{H}]^{+}$, calcd: 229.1223 , found: 229.1223 .

\section{Synthesis of M3 and M4}

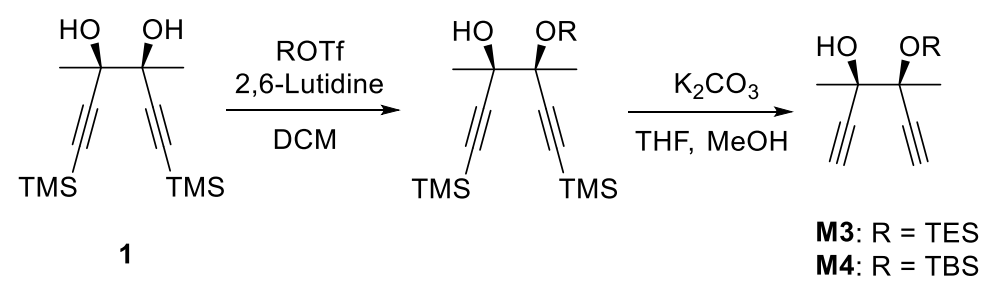

Compound 1 (720 mg, $2.5 \mathrm{mmol}$ ) was dissolved in DCM. Then, 2,6-lutidine (90 $\mu 1$, 0.77mmol) and triethylsilyl trifluoromethanesulfonate $(0.16 \mathrm{ml}, 0.77 \mathrm{mmol})$ was added sequentially at $0{ }^{\circ} \mathrm{C}$. After stirring $1 \mathrm{~h}$, the reaction was quenched by saturated $\mathrm{NH}_{4} \mathrm{Cl}$ aqueous solution. The organic layer was washed with brine and extracted by ethyl acetate, dried with $\mathrm{MgSO}_{4}$, and concentrated. This product was mixed with $\mathrm{K}_{2} \mathrm{CO}_{3}(67 \mathrm{mg})$, and dissolved in THF $(0.6 \mathrm{ml})$ and methanol $(0.6 \mathrm{ml})$. After $4 \mathrm{~h}$, the reaction was quenched by saturated $\mathrm{NH}_{4} \mathrm{Cl}$ aqueous solution. The organic layer was washed with brine and extracted by ethyl acetate, dried with $\mathrm{MgSO}_{4}$, and concentrated. The product was purified by flash column chromatography on silica gel to afford $\mathbf{M 3}$ as colorless liquid (88 mg, $0.35 \mathrm{mmol}, 45 \%$ via two steps). ${ }^{1} \mathrm{H} \mathrm{NMR}\left(500 \mathrm{MHz}, \mathrm{CDCl}_{3}\right) \delta 2.74(\mathrm{br}, 1 \mathrm{H}), 2.54(\mathrm{~s}, 1 \mathrm{H}), 2.41(\mathrm{~s}, 1 \mathrm{H})$, $1.54(\mathrm{~d}, J=1.6 \mathrm{~Hz}, 6 \mathrm{H}), 0.98(\mathrm{t}, J=7.9 \mathrm{~Hz}, 9 \mathrm{H}), 0.81-0.67(\mathrm{~m}, 6 \mathrm{H}) .{ }^{13} \mathrm{C} \mathrm{NMR}\left(125 \mathrm{MHz}, \mathrm{CDCl}_{3}\right) \delta 86.12,85.40,74.60,74.58$, 74.23, 72.13, 25.34, 24.05, 7.11, 6.04. HRMS (ESI): m/z for $\mathrm{C}_{14} \mathrm{H}_{24} \mathrm{NaO}_{2} \mathrm{Si}[\mathrm{M}+\mathrm{Na}]^{+}$, calcd: 275.1438, found: 275.1440 . 
Following the same procedure, compound 1 (2.07 g, $7.34 \mathrm{mmol})$ was reacted with tert-butyldimethylsilyl trifluoromethanesulfonate $(0.51 \mathrm{ml}, 2.20 \mathrm{mmol})$ to afford $\mathbf{M} 4$ as colorless liquid $\left(333 \mathrm{mg}, 1.32 \mathrm{mmol}, 60 \%\right.$ via two steps). ${ }^{1} \mathrm{H} \mathrm{NMR}(500 \mathrm{MHz}, \mathrm{CDCl} 3) \delta 2.71$ (br, 1H), $2.55(\mathrm{~s}, 1 \mathrm{H}), 2.41(\mathrm{~s}, 1 \mathrm{H}), 1.55(\mathrm{~s}, 3 \mathrm{H}), 1.54(\mathrm{~s}, 3 \mathrm{H}), 0.90(\mathrm{~s}, 9 \mathrm{H}), 0.24(\mathrm{~s}, 3 \mathrm{H}), 0.23(\mathrm{~s}, 3 \mathrm{H}) .{ }^{13} \mathrm{C} \mathrm{NMR}\left(125 \mathrm{MHz}, \mathrm{CDCl}_{3}\right)$ $\delta$ 86.02, 85.18, 74.90, 74.70, 74.22, 72.15, 25.82, 25.23, 24.07, 18.28, -2.97, -3.27. HRMS (ESI): m/z for $\mathrm{C}_{14} \mathrm{H}_{24} \mathrm{NaO}_{2} \mathrm{Si}[\mathrm{M}+\mathrm{Na}]^{+}$, calcd: 275.1438 , found 275.1439 .

\section{Synthesis of M5 and M6}

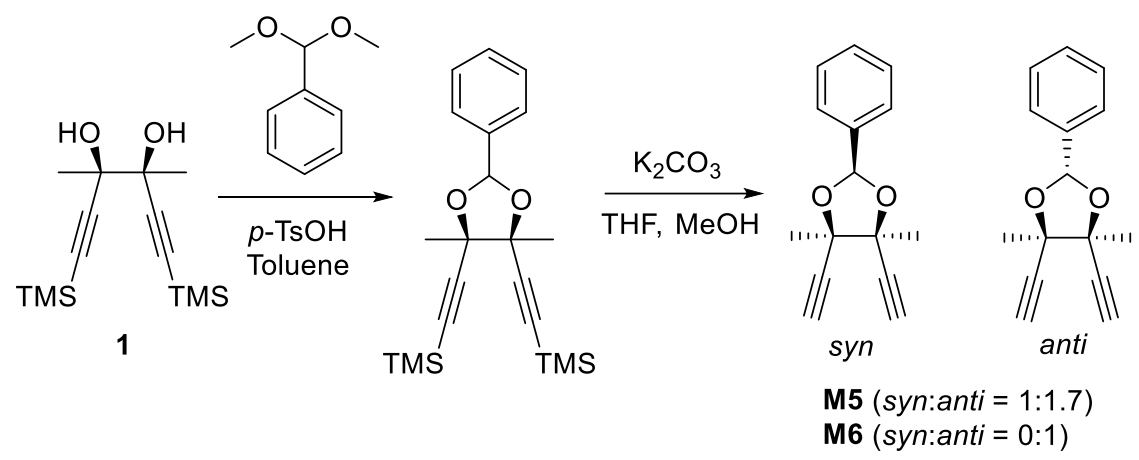

To a dried round-bottom flask containing a stirring bar, compound 1 (412 mg, $1.46 \mathrm{mmol}$ ) and $p$-toluenesulfonic acid (56 mg, 0.29 mmol) was added and purged with Ar gas. The mixture was dissolved in toluene $(1.5 \mathrm{ml})$, then benzaldehyde dimethyl acetal $(0.26$ $\mathrm{ml}, 1.75 \mathrm{mmol}$ ) was added. After stirring for $6 \mathrm{~h}$ at $70{ }^{\circ} \mathrm{C}$, the organic layer was washed with brine and extracted by ethyl acetate, dried with $\mathrm{MgSO}_{4}$, and concentrated. This crude mixture was dissolved in THF $(2 \mathrm{ml})$ and methanol $(2 \mathrm{ml})$, then $\mathrm{K}_{2} \mathrm{CO}_{3}(508 \mathrm{mg}$, $3.63 \mathrm{mmol}$ ) was added. After vigorous stirring for $3 \mathrm{~h}$, the reaction was quenched by saturated $\mathrm{NH}_{4} \mathrm{Cl}$ aqueous solution. The organic layer was washed with brine and extracted by ethyl acetate, dried with $\mathrm{MgSO}_{4}$, and concentrated. The product was purified by flash column chromatography on silica gel to afford M5 (syn:anti = 1:1.7) as colorless liquid (264 mg, 1.17 mmol, $80 \%$ via two steps). Further purification by recrystallization afforded M6 (syn:anti $=0: 1)$ as white solid $(90 \mathrm{mg}) .{ }^{1} \mathrm{H} \mathrm{NMR}(500 \mathrm{MHz}, \mathrm{CDCl} 3) \delta 7.54-$ $7.45(\mathrm{~m}, 2 \mathrm{H}), 7.42-7.35(\mathrm{~m}, 3 \mathrm{H}), 6.27(\mathrm{~s}, 1 \mathrm{H}), 2.70(\mathrm{~s}, 2 \mathrm{H}), 1.62(\mathrm{~s}, 6 \mathrm{H}) .{ }^{13} \mathrm{C} \mathrm{NMR}\left(125 \mathrm{MHz}, \mathrm{CDCl}_{3}\right) \delta 129.58,128.52,126.76$, 102.29, 82.64, 80.32, 75.51, 23.87. HRMS (ESI): $\mathrm{m} / \mathrm{z}$ for $\mathrm{C}_{15} \mathrm{H}_{14} \mathrm{NaO}_{2}[\mathrm{M}+\mathrm{Na}]^{+}$, calcd: 249.0886, found: 249.0887.

\section{Synthesis of M7-10}

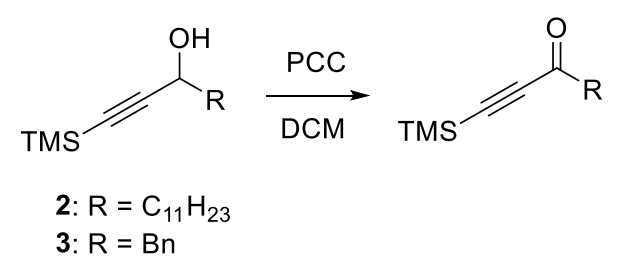

2: $\mathrm{R}=\mathrm{C}_{11} \mathrm{H}_{23}$

3: $\mathrm{R}=\mathrm{Bn}$
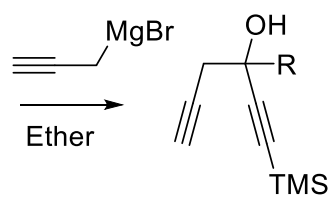

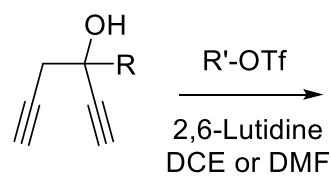

5: $\mathrm{R}=\mathrm{C}_{11} \mathrm{H}_{23}$
6: $\mathrm{R}=\mathrm{Bn}$

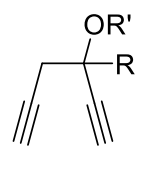

M7 $\left(\mathrm{R}=\mathrm{C}_{11} \mathrm{H}_{23}, \mathrm{R}^{\prime}=\right.$ TBS $)$
M8 $\left(\mathrm{R}=\mathrm{C}_{11} \mathrm{H}_{23}, \mathrm{R}^{\prime}=\right.$ TIPS $)$
M9 $\left(\mathrm{R}=\mathrm{Bn}, \mathrm{R}^{\prime}=\right.$ TBS $)$
M10 (R = Bn, $\mathrm{R}^{\prime}=$ TIPS $)$

Compound 2 (5.25 g, $18.6 \mathrm{mmol})$ was dissolved in DCM (62 ml), and pyridinium chlorochromate (6.01 g, $27.9 \mathrm{mmol})$ was added. After strring for $5 \mathrm{~h}$, the crude mixture was filtered with silica. Further purification with flash column chromatography afforded a mixture of the desired oxidized product and its TMS-deprotected derivative. A portion of this product (2.21 g) was dissolved in degassed diethyl ether $(30 \mathrm{ml})$, then propargyl magnesium bromide solution $(30 \mathrm{ml})^{*}$ was added at $-30{ }^{\circ} \mathrm{C}$. After checking the complete consumption of the starting materials by thin layer chromatography, the reaction was quenched by saturated $\mathrm{NH}_{4} \mathrm{Cl}$ 
aqueous solution. The organic layer was washed with brine and extracted by ethyl acetate, dried with $\mathrm{MgSO}_{4}$, and concentrated. This crude mixture was dissolved in THF $(10 \mathrm{ml})$ and methanol $(10 \mathrm{ml})$, then $\mathrm{K}_{2} \mathrm{CO}_{3}(5.9 \mathrm{~g}, 43 \mathrm{mmol})$ was added. After vigorous stirring for $2 \mathrm{~h}$, the reaction was quenched by saturated $\mathrm{NH}_{4} \mathrm{Cl}$ aqueous solution. The organic layer was washed with water and extracted by ethyl acetate, dried with $\mathrm{MgSO}_{4}$, and concentrated. The product was purified by flash column chromatography on silica gel to afford 5 as colorless liquid $\left(1.74 \mathrm{~g}, 7.0 \mathrm{mmol}\right.$, ca. $70 \%$ via three steps). ${ }^{1} \mathrm{H}$ NMR (500 MHz, $\left.\mathrm{CDCl}_{3}\right) \delta 2.62$ (ddd, $J=40.5$, 16.6, 2.6 Hz, 2H), 2.50 (s, 1H), 2.38 (br, 1H), $2.16(\mathrm{t}, J=2.6 \mathrm{~Hz}, 1 \mathrm{H}), 1.80-1.70(\mathrm{~m}, 2 \mathrm{H}), 1.55-1.49$ (m, $2 \mathrm{H}), 1.38-1.23(\mathrm{~m}$, $16 \mathrm{H}), 0.88(\mathrm{t}, J=6.9 \mathrm{~Hz}, 3 \mathrm{H}) .{ }^{13} \mathrm{C} \mathrm{NMR}\left(125 \mathrm{MHz}, \mathrm{CDCl}_{3}\right) \delta 85.52,79.40,72.82,72.06,69.70,40.97,33.33,32.07,29.79,29.77$, 29.75, 29.71, 29.65, 29.50, 24.41, 22.84, 14.28. HRMS (ESI): $\mathrm{m} / \mathrm{z}$ for $\mathrm{C}_{17} \mathrm{H}_{28} \mathrm{NaO}[\mathrm{M}+\mathrm{Na}]^{+}$, calcd: 271.2032, found: 271.2031 .

* Preparation of propargyl magnesium bromide (Grignard reagent)

To a two-neck round-bottom flask with a reflux condenser, magnesium turnings (2.2 g, 90 mmol) and mercury (II) chloride (610 $\mathrm{mg}, 2.3 \mathrm{mmol})$ was added. After drying it in vacuo using a torch, diethyl ether (30 ml) was added. Propargyl bromide solution ( $80 \%$ in toluene, $5.0 \mathrm{ml}, 45 \mathrm{mmol}$ ) was slowly added, then the reaction mixture is warmed over a water bath for $1 \mathrm{~h}$. After seeing bubbling stopped, the reagent was directly used for the Grignard reaction.

Following the same procedure, compound 3 was used to afford 6 as colorless liquid: ${ }^{1} \mathrm{H} \mathrm{NMR}\left(500 \mathrm{MHz}, \mathrm{CDCl}_{3}\right) \delta 7.39-7.27(\mathrm{~m}$, $5 \mathrm{H}), 3.11(\mathrm{q}, J=13.4 \mathrm{~Hz}, 2 \mathrm{H}), 2.64(\mathrm{~d}, J=2.6 \mathrm{~Hz}, 2 \mathrm{H}), 2.54(\mathrm{~s}, 1 \mathrm{H}), 2.42(\mathrm{~s}, 1 \mathrm{H}), 2.22(\mathrm{t}, J=2.6 \mathrm{~Hz}, 1 \mathrm{H}) .{ }^{13} \mathrm{C} \mathrm{NMR}(125 \mathrm{MHz}$, $\left.\mathrm{CDCl}_{3}\right) \delta 135.41,130.89,128.36,127.37,85.12,79.41,74.18,72.33,69.72,46.58,32.63 . \mathrm{HRMS}(\mathrm{ESI}): \mathrm{m} / \mathrm{z}$ for $\mathrm{C}_{13} \mathrm{H}_{12} \mathrm{NaO}$ $[\mathrm{M}+\mathrm{Na}]^{+}$, calcd: 207.0780, found: 207.0783 .

Compound 5 (150 mg, $0.61 \mathrm{mmol})$ was dissolved in DCE (3 ml). Then, 2,6-lutidine (0.35 ml, $3.0 \mathrm{mmol})$ and tert-butyldimethylsilyl trifluoromethanesulfonate $(0.42 \mathrm{ml}, 1.8 \mathrm{mmol})$ was added. After stirring for $5 \mathrm{~h}$ at $50{ }^{\circ} \mathrm{C}$, the reaction was quenched by saturated $\mathrm{NaHCO}_{3}$ aqueous solution. The organic layer was washed with water and extracted by dichloromethane, dried with $\mathrm{MgSO}_{4}$, and concentrated. The product was purified by flash column chromatography on silica gel to afford M7 as colorless liquid (188 mg, $0.518 \mathrm{mmol}, 85 \%) .{ }^{1} \mathrm{H}$ NMR $\left(500 \mathrm{MHz}, \mathrm{CDCl}_{3}\right) \delta 2.56(\mathrm{dd}, J=2.5,1.5 \mathrm{~Hz}, 2 \mathrm{H}), 2.50(\mathrm{~s}, 1 \mathrm{H}), 2.04(\mathrm{t}, J=2.6 \mathrm{~Hz}, 1 \mathrm{H}), 1.81-1.71$ $(\mathrm{m}, 2 \mathrm{H}), 1.51-1.39(\mathrm{~m}, 2 \mathrm{H}), 1.37-1.19(\mathrm{~m}, 17 \mathrm{H}), 0.91-0.85(\mathrm{~m}, 12 \mathrm{H}), 0.19(\mathrm{~d}, J=8.9 \mathrm{~Hz}, 6 \mathrm{H}) .{ }^{13} \mathrm{C} \mathrm{NMR}(125 \mathrm{MHz}, \mathrm{CDCl}) \delta$ $86.41,80.37,73.70,70.99,70.77,42.14,33.80,32.08,29.80,29.76,29.73,29.69,29.51,25.84,24.14,22.85,18.33,14.28,-2.84,-$ 2.95. HRMS (ESI): $\mathrm{m} / \mathrm{z}$ for $\mathrm{C}_{23} \mathrm{H}_{42} \mathrm{NaOSi}[\mathrm{M}+\mathrm{Na}]^{+}$, calcd: 385.2897 , found: 385.2899 .

Following the similar procedure with different reaction times, M8-M10 were obtained from compounds 5 and 6.

From the reaction of compound $5(550 \mathrm{mg}, 2.2 \mathrm{mmol})$ with triisopropylsilyl trifluoromethanesulfonate $(1.8 \mathrm{ml}, 6.6 \mathrm{mmol}), \mathbf{M 8} \mathrm{was}$ obtained as colorless liquid (818 mg, $2.02 \mathrm{mmol}, 91 \%) .{ }^{1} \mathrm{H} \mathrm{NMR}\left(500 \mathrm{MHz}, \mathrm{CDCl}_{3}\right) \delta 2.64(\mathrm{~d}, J=2.7 \mathrm{~Hz}, 2 \mathrm{H}), 2.47(\mathrm{~s}, 1 \mathrm{H}), 2.03$ $(\mathrm{t}, J=2.7 \mathrm{~Hz}, 1 \mathrm{H}), 1.93-1.81(\mathrm{~m}, 2 \mathrm{H}), 1.49-1.42(\mathrm{~m}, 2 \mathrm{H}), 1.40-1.21(\mathrm{~m}, 18 \mathrm{H}), 1.21-1.14(\mathrm{~m}, 3 \mathrm{H}), 1.08(\mathrm{dd}, J=7.3,2.4 \mathrm{~Hz}$, $18 \mathrm{H}), 0.88(\mathrm{t}, J=7.0 \mathrm{~Hz}, 3 \mathrm{H}) .{ }^{13} \mathrm{C} \mathrm{NMR}\left(125 \mathrm{MHz}, \mathrm{CDCl}_{3}\right) \delta 86.85,80.31,73.22,70.89,70.79,42.01,33.11,32.08,29.80,29.79$, 29.71, 29.67, 29.51, 24.09, 22.85, 18.51, 14.28, 13.18. HRMS (ESI): $\mathrm{m} / \mathrm{z}$ for $\mathrm{C}_{26} \mathrm{H}_{48} \mathrm{NaOSi}[\mathrm{M}+\mathrm{Na}]^{+}$, calcd: 427.3367, found: 427.3369 .

From the reaction of compound $6(109 \mathrm{mg}, 0.59 \mathrm{mmol})$ with tert-butyldimethylsilyl trifluoromethanesulfonate $(0.54 \mathrm{ml}, 2.4 \mathrm{mmol})$, M9 was obtained as colorless liquid (138 mg, $0.46 \mathrm{mmol}, 78 \%$ ). ${ }^{1} \mathrm{H} \mathrm{NMR}\left(500 \mathrm{MHz}, \mathrm{CDCl}_{3}\right) \delta 7.38(\mathrm{~d}, J=6.9 \mathrm{~Hz}, 2 \mathrm{H}), 7.33-7.24$ $(\mathrm{m}, 3 \mathrm{H}), 3.10(\mathrm{qd}, J=13.2,2.3 \mathrm{~Hz}, 2 \mathrm{H}), 2.58(\mathrm{~d}, J=2.9 \mathrm{~Hz}, 1 \mathrm{H}), 2.56(\mathrm{~s}, 2 \mathrm{H}), 2.16(\mathrm{q}, J=2.6 \mathrm{~Hz}, 1 \mathrm{H}), 0.88(\mathrm{~d}, J=2.8 \mathrm{~Hz}, 9 \mathrm{H})$, $0.17(\mathrm{~d}, J=2.6 \mathrm{~Hz}, 3 \mathrm{H}),-0.06(\mathrm{~d}, J=2.6 \mathrm{~Hz}, 3 \mathrm{H}) .{ }^{13} \mathrm{C} \mathrm{NMR}\left(125 \mathrm{MHz}, \mathrm{CDCl}_{3}\right) \delta 136.34,131.35,127.75,126.83,85.75,80.38$, 75.45, 71.53, 71.28, 47.76, 33.68, 25.96, 18.37, -2.80, -3.35. HRMS (ESI): m/z for $\mathrm{C}_{19} \mathrm{H}_{26} \mathrm{NaOSi}[\mathrm{M}+\mathrm{Na}]^{+}$, calcd: 321.1645, found: 321.1648 . 
From the reaction of compound $6(150 \mathrm{mg}, 0.83 \mathrm{mmol})$ with triisopropylsilyl trifluoromethanesulfonate $(0.90 \mathrm{ml}, 3.3 \mathrm{mmol}) \mathrm{in}$ DMF $\left(80{ }^{\circ} \mathrm{C}, 40 \mathrm{~h}\right), \mathbf{M 1 0}$ was obtained as colorless liquid $(143 \mathrm{mg}, 0.42 \mathrm{mmol}, 51 \%) .{ }^{1} \mathrm{H} \mathrm{NMR}\left(500 \mathrm{MHz}, \mathrm{CDCl}_{3}\right) \delta 7.41(\mathrm{~d}, J=$ $6.8 \mathrm{~Hz}, 2 \mathrm{H}), 7.32-7.22(\mathrm{~m}, 3 \mathrm{H}), 3.20(\mathrm{dd}, J=40.7,13.2 \mathrm{~Hz}, 2 \mathrm{H}), 2.57-2.52(\mathrm{~m}, 3 \mathrm{H}), 2.17(\mathrm{t}, J=2.6 \mathrm{~Hz}, 1 \mathrm{H}), 1.25-1.16(\mathrm{~m}$, $3 \mathrm{H}), 1.07$ (dd, $J=7.4,4.4 \mathrm{~Hz}, 18 \mathrm{H}) .{ }^{13} \mathrm{C} \mathrm{NMR}\left(125 \mathrm{MHz}, \mathrm{CDCl}_{3}\right) \delta$ 136.28, 131.21, 127.90, 126.89, 86.23, 80.52, 74.95, 71.77, 71.26, 47.39, 32.75, 18.53, 18.49, 13.20. HRMS (ESI): $\mathrm{m} / \mathrm{z}$ for $\mathrm{C}_{22} \mathrm{H}_{32} \mathrm{NaOSi}[\mathrm{M}+\mathrm{Na}]^{+}$, calcd: 363.2115 , found: 363.2117 .

\section{Preparation of Ru3}<smiles>[R6]N1CCN([PbH])C1[R]([R6])(Cl)Cl</smiles><smiles></smiles>

Ru3

Under argon atmosphere, 3-chloropyridine (0.4 ml, $4.2 \mathrm{mmol})$ was added to a solution of dichloro[1,3-bis(2,6-isopropylphenyl)-2imidazolidinylidene](benzylidene)(tricyclohexylphosphine)ruthenium(II) (59.4 mg, $0.0636 \mathrm{mmol})$ in toluene (0.33 ml). After stirring for $2 \mathrm{~min}$, the solution was mixed with degassed n-pentane (ca. $8 \mathrm{ml}$ ). A small amount of green precipitate formed immediately, and the vial was stored at $-24{ }^{\circ} \mathrm{C}$ for 2 days. Dark green crystals formed and were isolated by filtration, washed with pentane, and then dried in vacuo (34 mg, 61\%). ${ }^{1} \mathrm{H} \mathrm{NMR}\left(500 \mathrm{MHz}, \mathrm{CD}_{2} \mathrm{Cl}_{2}\right) \delta 19.00(\mathrm{~s}, 1 \mathrm{H}), 8.56(\mathrm{br} \mathrm{d}, J=45.3 \mathrm{~Hz}, 2 \mathrm{H}), 7.99(\mathrm{br}$ $\mathrm{d}, J=28.4 \mathrm{~Hz}, 2 \mathrm{H}), 7.76$ (br s, 1H), 7.55 (br s, 1H), $7.52-7.42$ (m, 3H), $7.41-7.13$ (m, 7H), $7.09-6.90$ (m, 3H), $4.61-3.07$ (m, 8H), $1.87-0.93(\mathrm{~m}, 28 \mathrm{H}) .{ }^{13} \mathrm{C} \mathrm{NMR}\left(125 \mathrm{MHz}, \mathrm{CD}_{2} \mathrm{Cl}_{2}\right) \delta 218.73,152.10,151.55,149.19,148.04,137.00,136.08,130.39,129.35$, 128.37, 124.73, 55.09, 29.23, 28.51, 27.49, 26.62, 23.58. Anal. Calcd. for $\mathrm{C}_{44} \mathrm{H}_{52} \mathrm{Cl}_{4} \mathrm{~N}_{4} \mathrm{Ru}$ : C, 60.07; $\mathrm{H}, 5.96 ; \mathrm{Cl}, 16.12 ; \mathrm{N}, 6.37 ; \mathrm{Ru}$, 11.49. Found: C, 60.07; H, 6.06; N, 6.39.

\section{General Procedure for Polymerization}

A 4-mL sized screw-cap vial with septum was flame dried and charged with monomer (ca. 20 mg). The vial was purged with Ar three times, and degassed anhydrous solvent was added. A mixture of initiator and additive in another 4-mL vial was dissolved in solvent under Ar atmosphere. The initiator solution was rapidly injected to the monomer solution at experimental temperature under vigorous stirring. The reaction was quenched by excess ethyl vinyl ether (ca. $50-100 \mu \mathrm{l}$ ) after desired reaction time, and resulting polymer was precipitated in methanol, remaining small amount of crude mixture (c.a. $2 \mathrm{mg}$ ). Obtained solid was filtered and dried in vacuo. Monomer conversion was calculated from the ${ }^{1} \mathrm{H}$ NMR spectrum of the remained crude mixture.

\section{5. ${ }^{1} \mathrm{H}$ and ${ }^{13} \mathrm{C}$ NMR characterization of polymers}

P3: ${ }^{1} \mathrm{H}$ NMR $\left(500 \mathrm{MHz}, \mathrm{CD}_{2} \mathrm{Cl}_{2}\right) \delta 6.83-6.27(\mathrm{~m}, 2 \mathrm{H}), 3.12-2.89(\mathrm{~m}, 1 \mathrm{H}), 1.58(\mathrm{~s}, 3 \mathrm{H}), 1.43(\mathrm{~s}, 3 \mathrm{H}), 1.06-0.93(\mathrm{~m}, 9 \mathrm{H}), 0.79$ - $0.64(\mathrm{~m}, 6 \mathrm{H}) .{ }^{13} \mathrm{C} \mathrm{NMR}\left(150 \mathrm{MHz}, \mathrm{CDCl}_{3}\right) \delta 148.45,145.75,123.57,122.94,82.71,79.18,20.91,19.91,7.20,6.72$.

P4: ${ }^{1} \mathrm{H}$ NMR $\left(500 \mathrm{MHz}, \mathrm{CD}_{2} \mathrm{Cl}_{2}\right) \delta 6.79-6.29(\mathrm{~m}, 2 \mathrm{H}), 3.06-2.79(\mathrm{~m}, 1 \mathrm{H}), 1.58(\mathrm{br}, 3 \mathrm{H}), 1.42(\mathrm{~s}, 3 \mathrm{H}), 0.91(\mathrm{~s}, 9 \mathrm{H}), 0.38-0.12$ $(\mathrm{m}, 6 \mathrm{H}) .{ }^{13} \mathrm{C} \mathrm{NMR}\left(150 \mathrm{MHz}, \mathrm{CDCl}_{3}\right) \delta 148.16,145.76,123.34,123.02,82.78,79.60,26.06,20.88,19.96,18.48,-2.25$. 
P6: ${ }^{1} \mathrm{H}$ NMR (500 MHz, $\left.\mathrm{CD}_{2} \mathrm{Cl}_{2}\right) \delta 7.47(\mathrm{br}, 2 \mathrm{H}), 7.36(\mathrm{~d}, J=12.1 \mathrm{~Hz}, 3 \mathrm{H}), 6.64(\mathrm{~s}, 2 \mathrm{H}), 5.64(\mathrm{~s}, 1 \mathrm{H}), 1.54(\mathrm{~s}, 6 \mathrm{H}) .{ }^{13} \mathrm{C} \mathrm{NMR}(125$ $\left.\mathrm{MHz}, \mathrm{CD}_{2} \mathrm{Cl}_{2}\right) \delta 142.52,136.99,129.80,128.58,127.80,124.03,100.77,87.82,16.92$.

P7: ${ }^{1} \mathrm{H}$ NMR $\left(500 \mathrm{MHz}, \mathrm{CD}_{2} \mathrm{Cl}_{2}\right) \delta 6.74(\mathrm{~d}, J=13.7 \mathrm{~Hz}, 1 \mathrm{H}), 6.25(\mathrm{~d}, J=15.5 \mathrm{~Hz}, 1 \mathrm{H}), 3.06-2.26(\mathrm{~m}, 2 \mathrm{H}), 1.96(\mathrm{br}, 1 \mathrm{H}), 1.73-$ $1.08(\mathrm{~m}, 19 \mathrm{H}), 1.08-0.61(\mathrm{~m}, 12 \mathrm{H}), 0.38--0.41(\mathrm{~m}, 6 \mathrm{H}) .{ }^{13} \mathrm{C}$ NMR $\left(125 \mathrm{MHz}, \mathrm{CD}_{2} \mathrm{Cl}_{2}\right) \delta 146.73,140.06,124.17,121.99,79.10$, $41.99,40.66,32.38,30.44,30.11,29.81,26.26,26.19,23.14,18.67,14.35,-2.51,-3.36$.

P8: ${ }^{1} \mathrm{H}$ NMR $\left(500 \mathrm{MHz}, \mathrm{CD}_{2} \mathrm{Cl}_{2}\right) \delta 6.80(\mathrm{~d}, J=12.7 \mathrm{~Hz}, 1 \mathrm{H}), 6.24(\mathrm{~d}, J=12.7 \mathrm{~Hz}, 1 \mathrm{H}), 2.76(\mathrm{~s}, 1 \mathrm{H}), 2.60(\mathrm{~s}, 1 \mathrm{H}), 1.95(\mathrm{~s}, J=84.6$ $\mathrm{Hz}, 1 \mathrm{H}), 1.75(\mathrm{~s}, 1 \mathrm{H}), 1.65-1.12(\mathrm{~m}, 18 \mathrm{H}), 1.05(\mathrm{~s}, 18 \mathrm{H}), 0.88(\mathrm{t}, J=6.1 \mathrm{~Hz}, 3 \mathrm{H}) .{ }^{13} \mathrm{C} \mathrm{NMR}\left(125 \mathrm{MHz}, \mathrm{CD}_{2} \mathrm{Cl}_{2}\right) \delta 147.26,140.04$, $123.97,121.88,78.74,42.44,41.10,40.68,32.39,30.51,30.10,29.81,25.54,23.15,18.64,14.35,13.61$.

P9: ${ }^{1} \mathrm{H}$ NMR (500 MHz, $\left.\mathrm{CD}_{2} \mathrm{Cl}_{2}\right) \delta 7.48-6.89(\mathrm{~m}, 5 \mathrm{H}), 6.54-6.11(\mathrm{~m}, 1 \mathrm{H}), 6.11-5.84(\mathrm{~m}, 1 \mathrm{H}), 3.44-2.94(\mathrm{~m}, 2 \mathrm{H}), 2.94-2.27$ $(\mathrm{m}, 2 \mathrm{H}), 1.12-0.56(\mathrm{~m}, 9 \mathrm{H}), 0.28--0.33(\mathrm{~m}, 6 \mathrm{H}) .{ }^{13} \mathrm{C} \mathrm{NMR}\left(150 \mathrm{MHz}, \mathrm{CD}_{2} \mathrm{Cl}_{2}\right) \delta 146.53,140.20,138.96,131.30,127.95,126.64$, $124.43,122.14,79.30,47.32,41.83,26.34,18.69,-2.59,-3.44$.

P10: ${ }^{1} \mathrm{H}$ NMR $\left(500 \mathrm{MHz}, \mathrm{THF}-d_{8}\right) \delta 7.60-6.76(\mathrm{~m}, 5 \mathrm{H}), 6.37(\mathrm{br}, 1 \mathrm{H}), 6.03(\mathrm{br}, 1 \mathrm{H}), 3.45-2.98(\mathrm{~m}, 2 \mathrm{H}), 2.97-2.53(\mathrm{~m}, 2 \mathrm{H})$, 1.24 (br, 21H). ${ }^{13} \mathrm{C}$ NMR $\left(125 \mathrm{MHz}, \mathrm{THF}-d_{8}\right) \delta 146.72,140.32,138.73,131.53,128.12,126.94,124.39,122.43,79.35,54.69,47.65$, $42.53,18.82,13.99$.

\section{General procedure for In situ NMR Experiment}

An NMR tube was filled with monomer $(0.125 \mathrm{mmol}, 10 \mathrm{eq})$, purged with argon, and THF- $d_{8}(300 \mu \mathrm{L})$ was added. A $4-\mathrm{mL}$ vial containing initiator $(0.0156 \mathrm{mmol}, 1.25 \mathrm{eq})$ was argon-purged, and hexamethyldisilane was added as an internal standard. The total amount of initiator was $5 / 4$ of the amount used for the reaction; after dissolving those using THF- $d_{8}(250 \mu \mathrm{L}), 1 / 5(50 \mu \mathrm{L})$ of it was diluted in another NMR tube and used for checking the ratio between initial carbene and the internal standard. Then, the remaining $200 \mu \mathrm{L}$ of initiator solution was added to the monomer solution and ${ }^{1} \mathrm{H}$ NMR was recorded over time. 

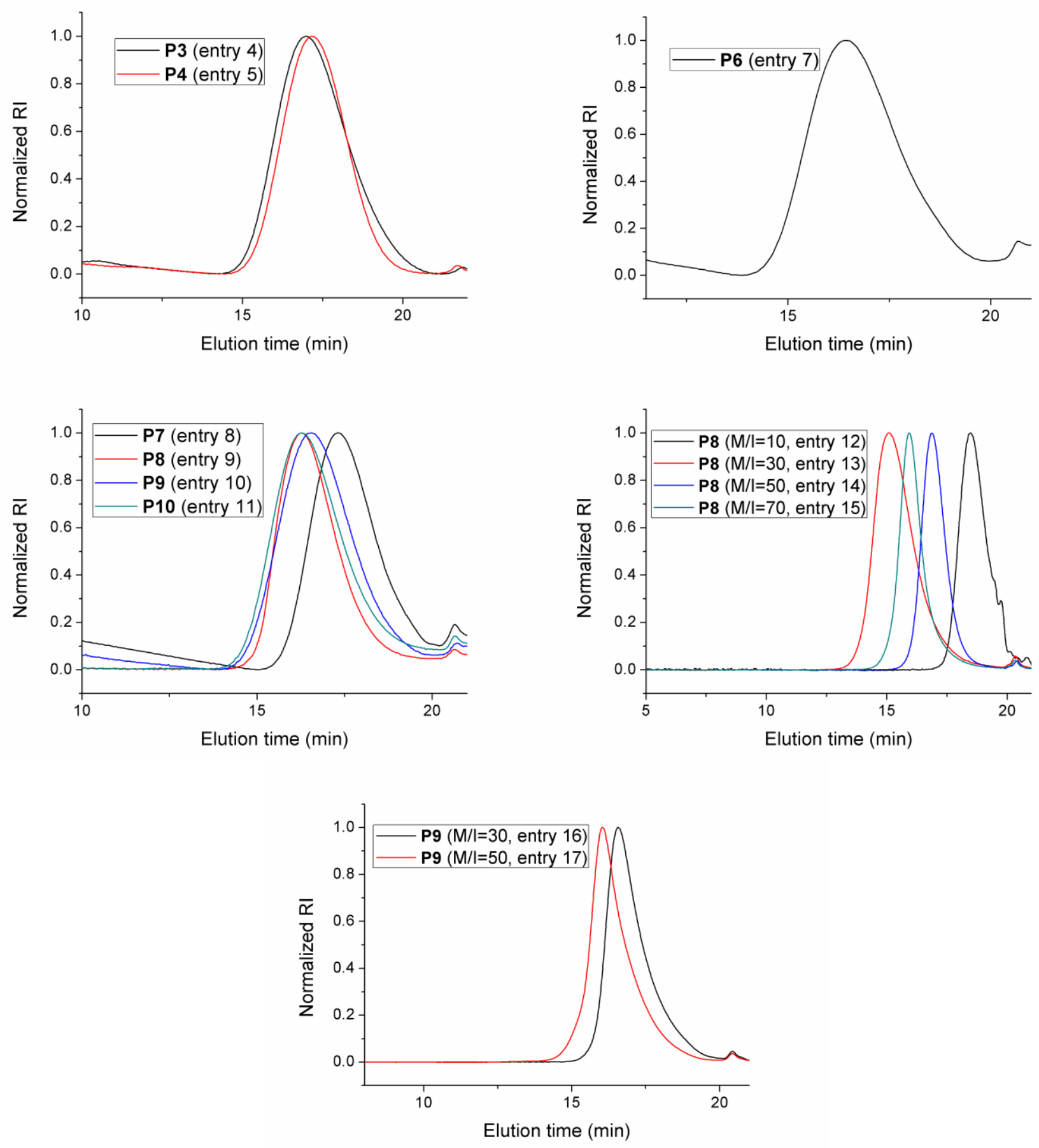

Figure S15. SEC traces of P3 - P10 from Table 1. 
8. ${ }^{1} \mathrm{H}$ and ${ }^{13} \mathrm{C}$ NMR Spectra of New Compounds

M2 ${ }^{1} \mathrm{H}$ NMR $\left(400 \mathrm{MHz}, \mathrm{CDCl}_{3}\right)$

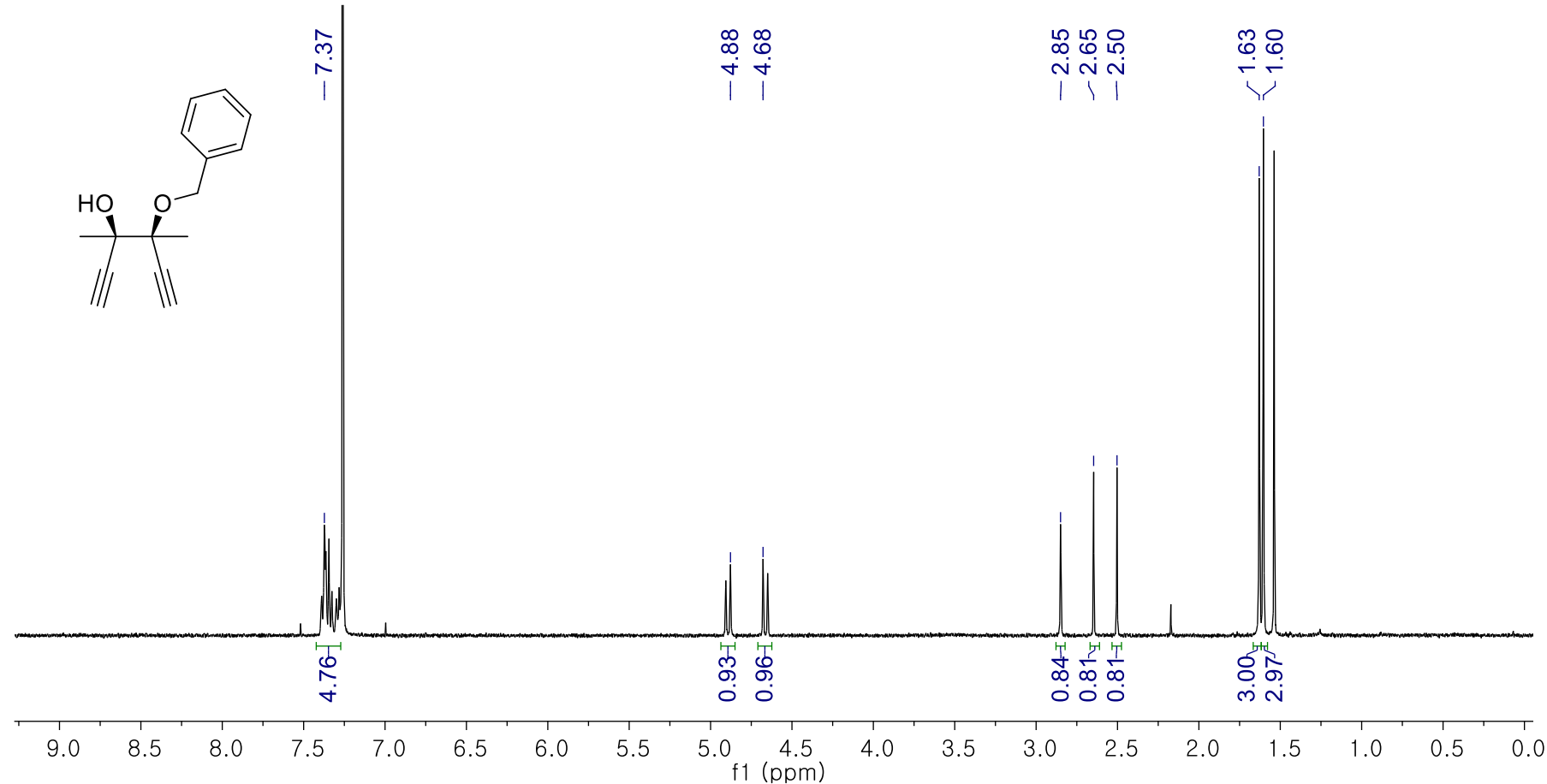

M2 ${ }^{13} \mathrm{C}$ NMR $\left(125 \mathrm{MHz}, \mathrm{CDCl}_{3}\right)$

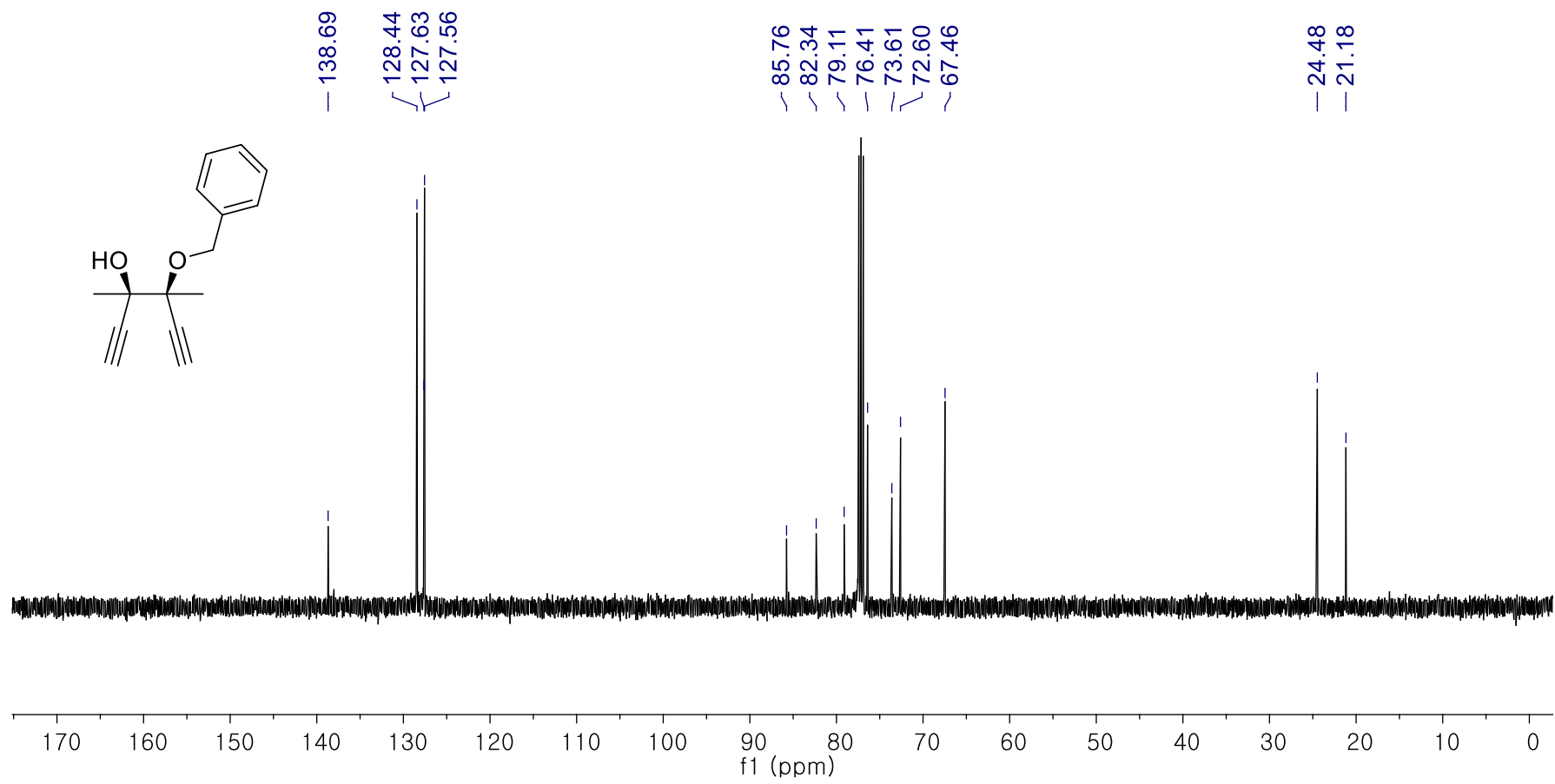




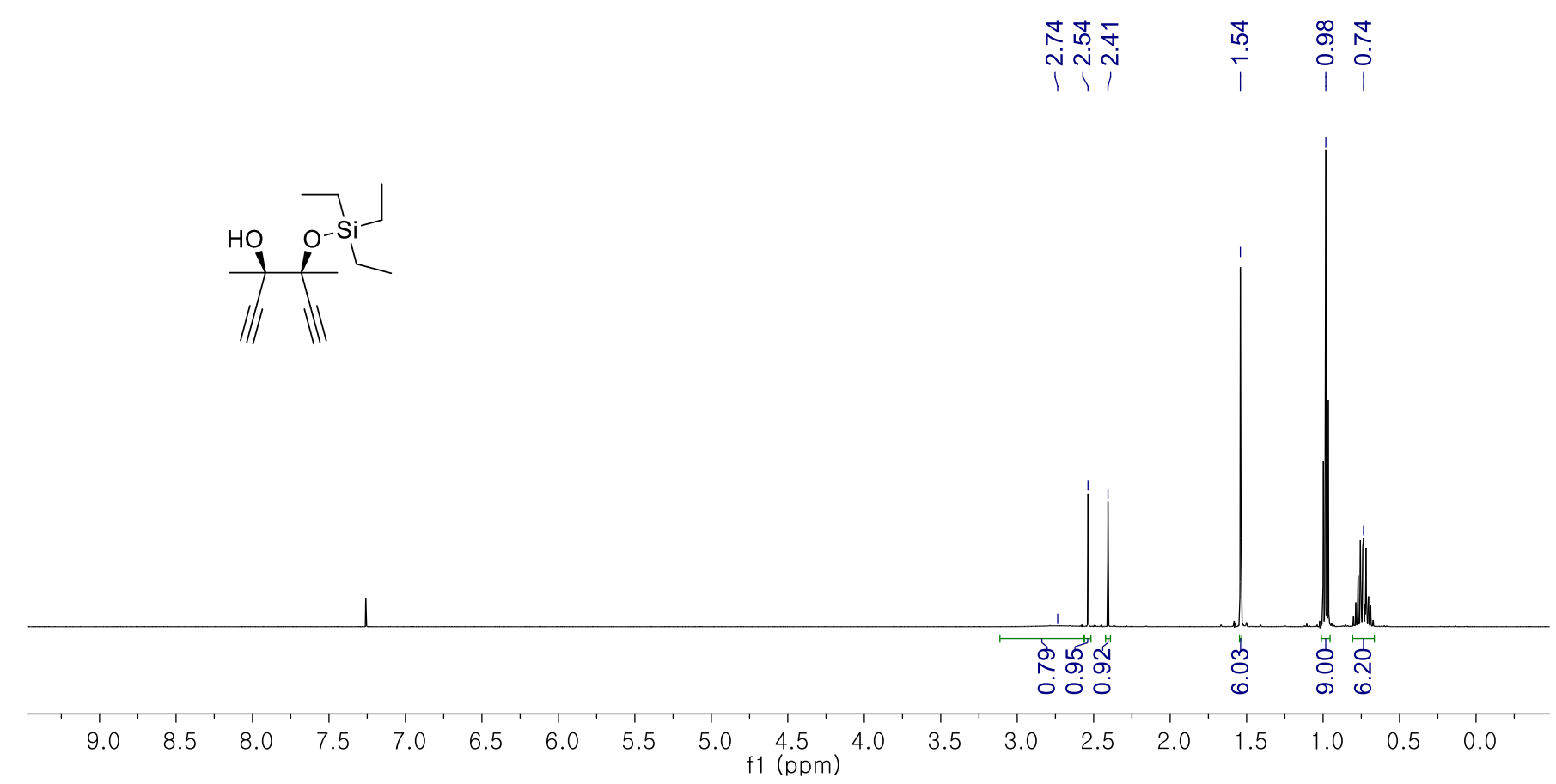

M3 ${ }^{13} \mathrm{C}$ NMR $\left(125 \mathrm{MHz}, \mathrm{CDCl}_{3}\right)$

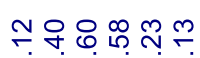

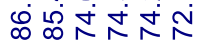

ir
芦

ผู่

동<smiles>C#CC(C)(O)C(C#C)(CC)O[Si](CC)(CC)CC</smiles>

23

210

190

170

150

130

110

9080

$70 \quad 60 \quad 50$

f1 (ppm) 
P3 ${ }^{1} \mathrm{H}$ NMR (500 MHz, $\left.\mathrm{CD}_{2} \mathrm{Cl}_{2}\right)$

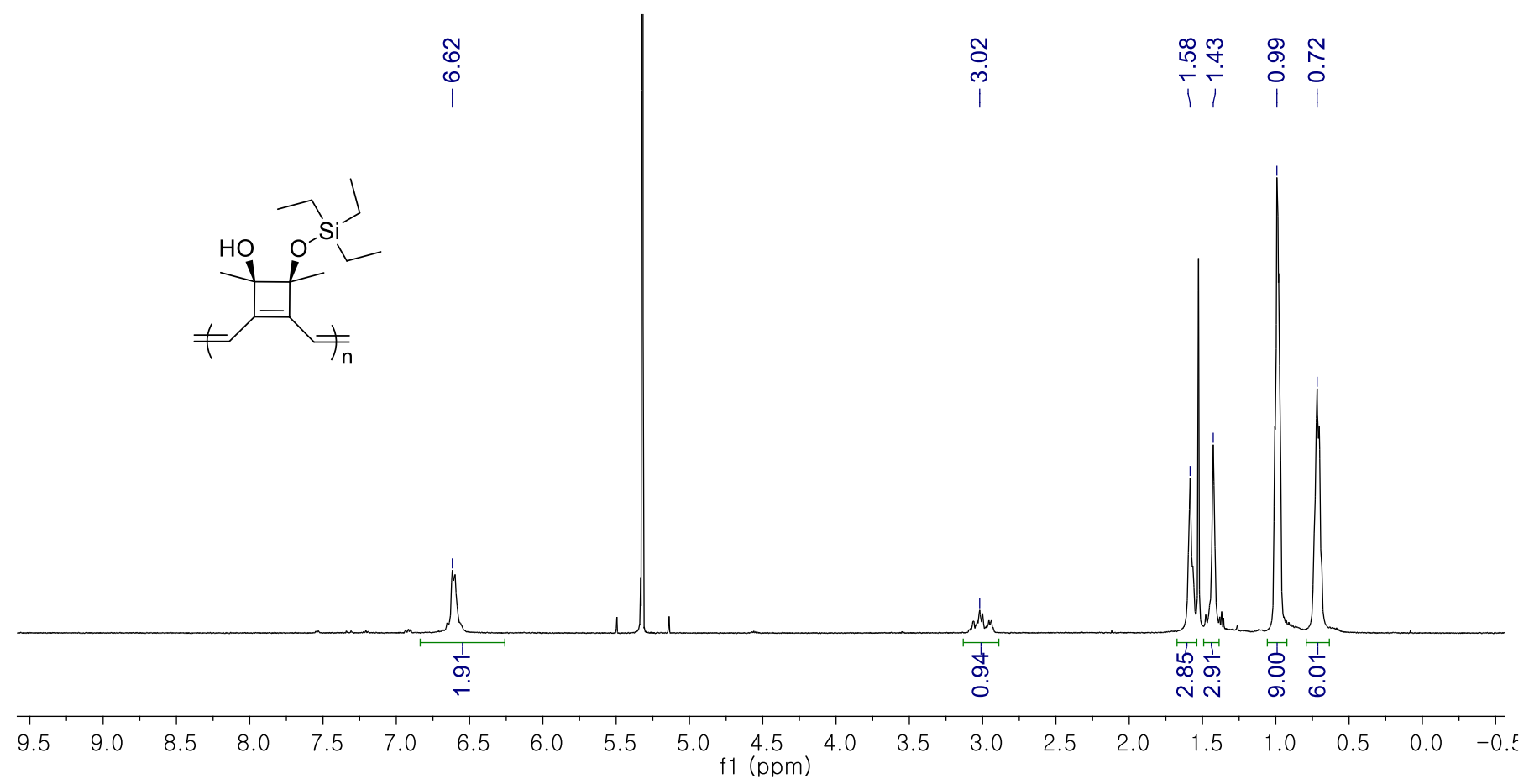

P3 ${ }^{13} \mathrm{C}$ NMR $\left(150 \mathrm{MHz}, \mathrm{CDCl}_{3}\right)$

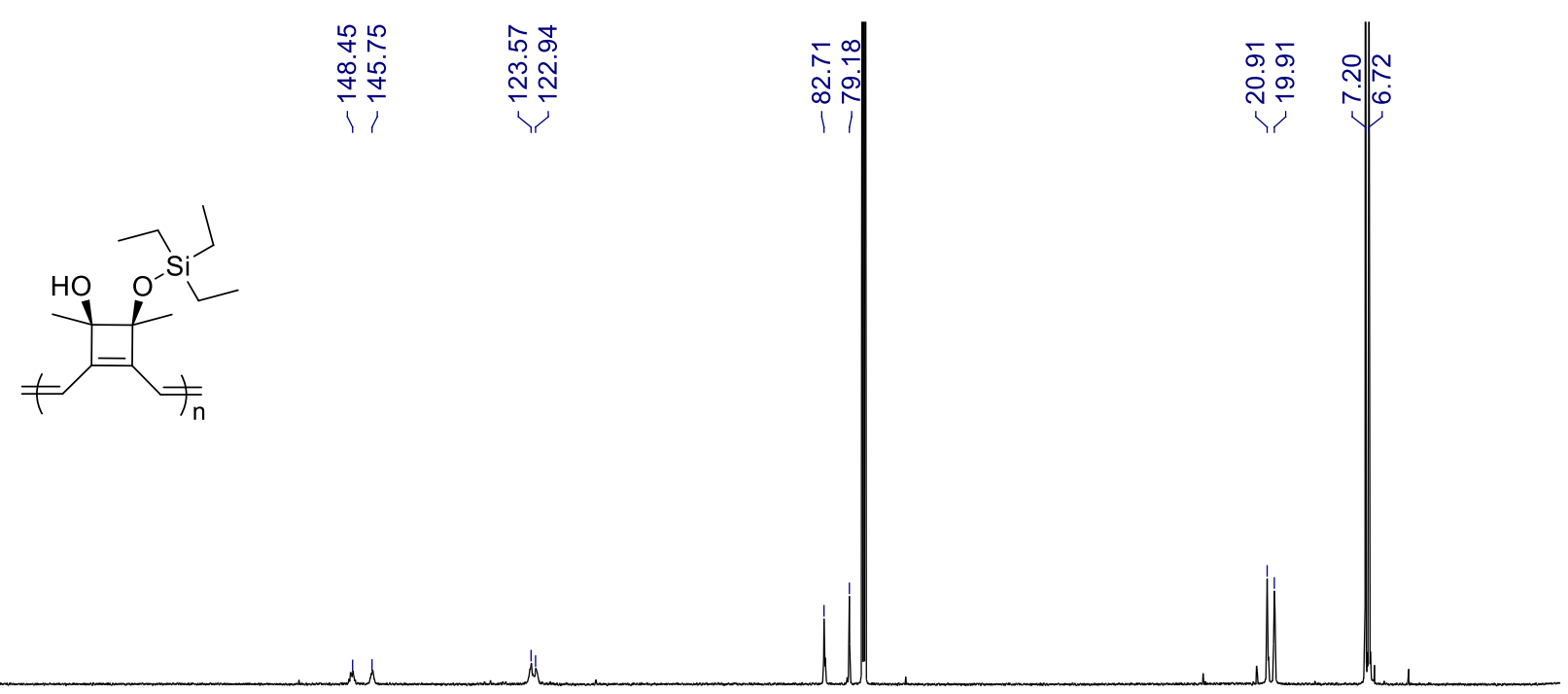

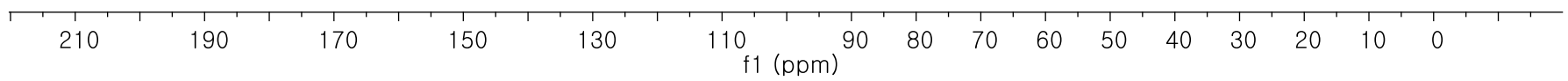




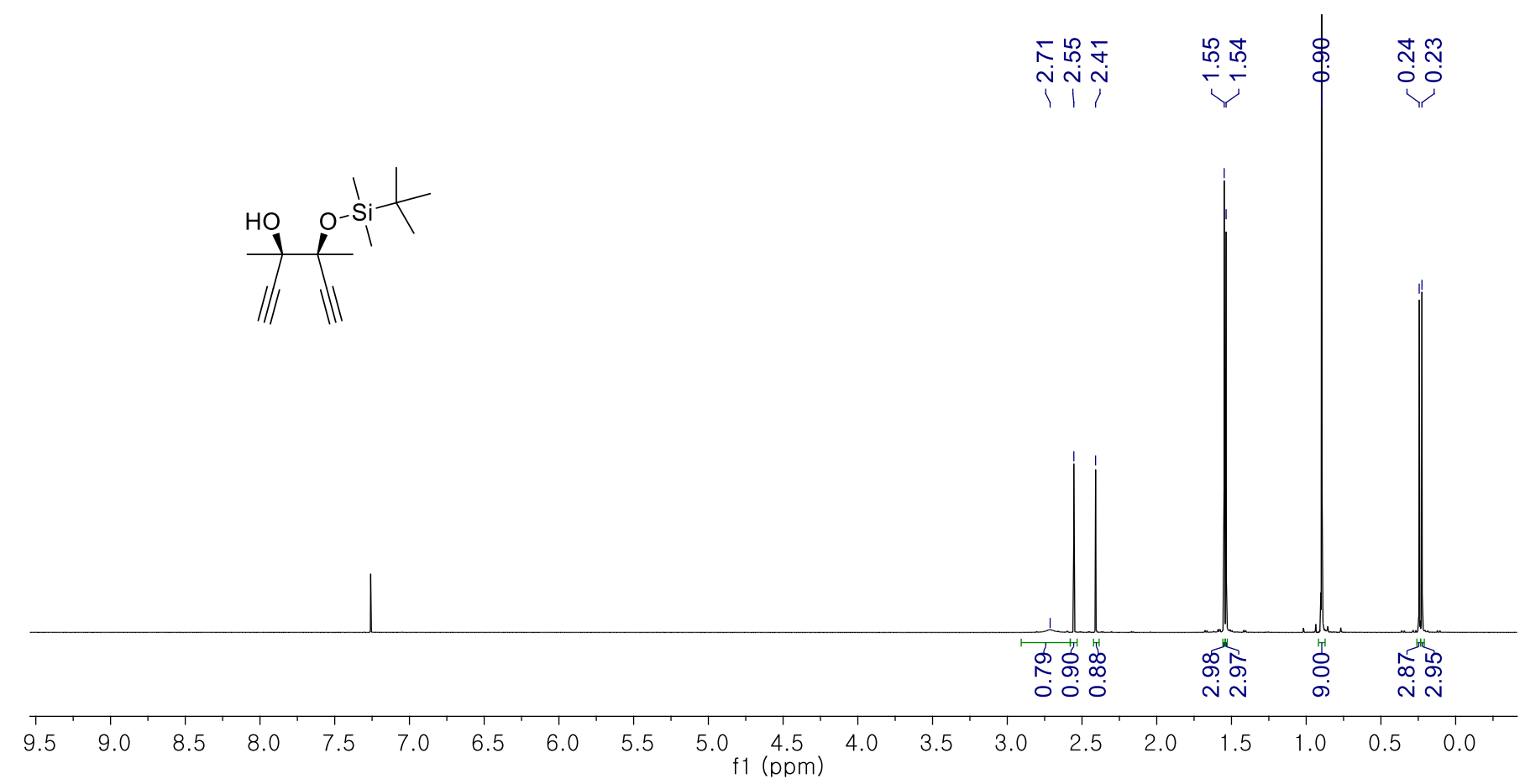

M4 ${ }^{13} \mathrm{C}$ NMR (125 MHz, $\left.\mathrm{CDCl}_{3}\right)$

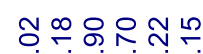

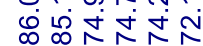

i, Vi,

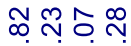

ஸู่

औi
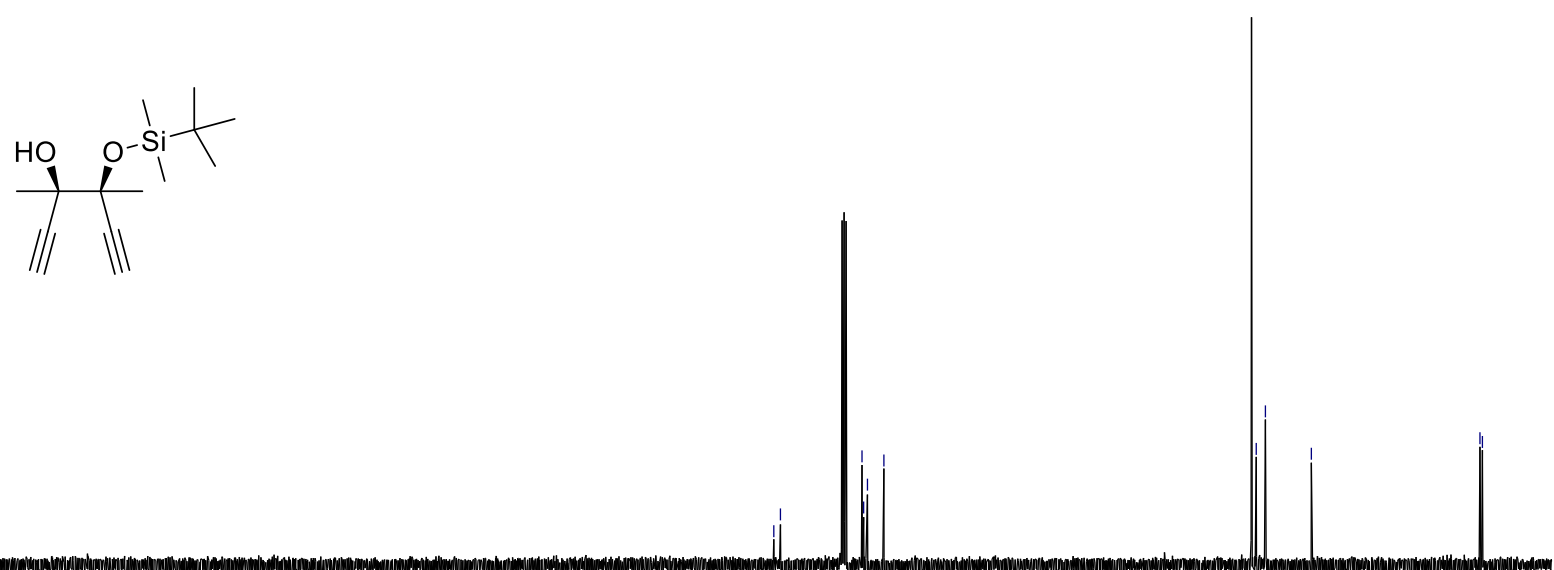

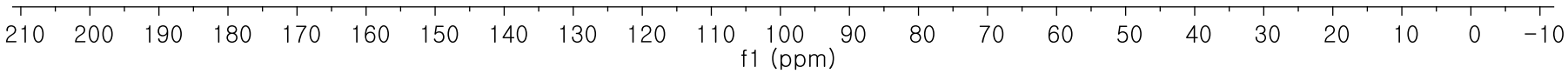


P4 ${ }^{1} \mathrm{H}$ NMR (500 MHz, $\left.\mathrm{CD}_{2} \mathrm{Cl}_{2}\right)$

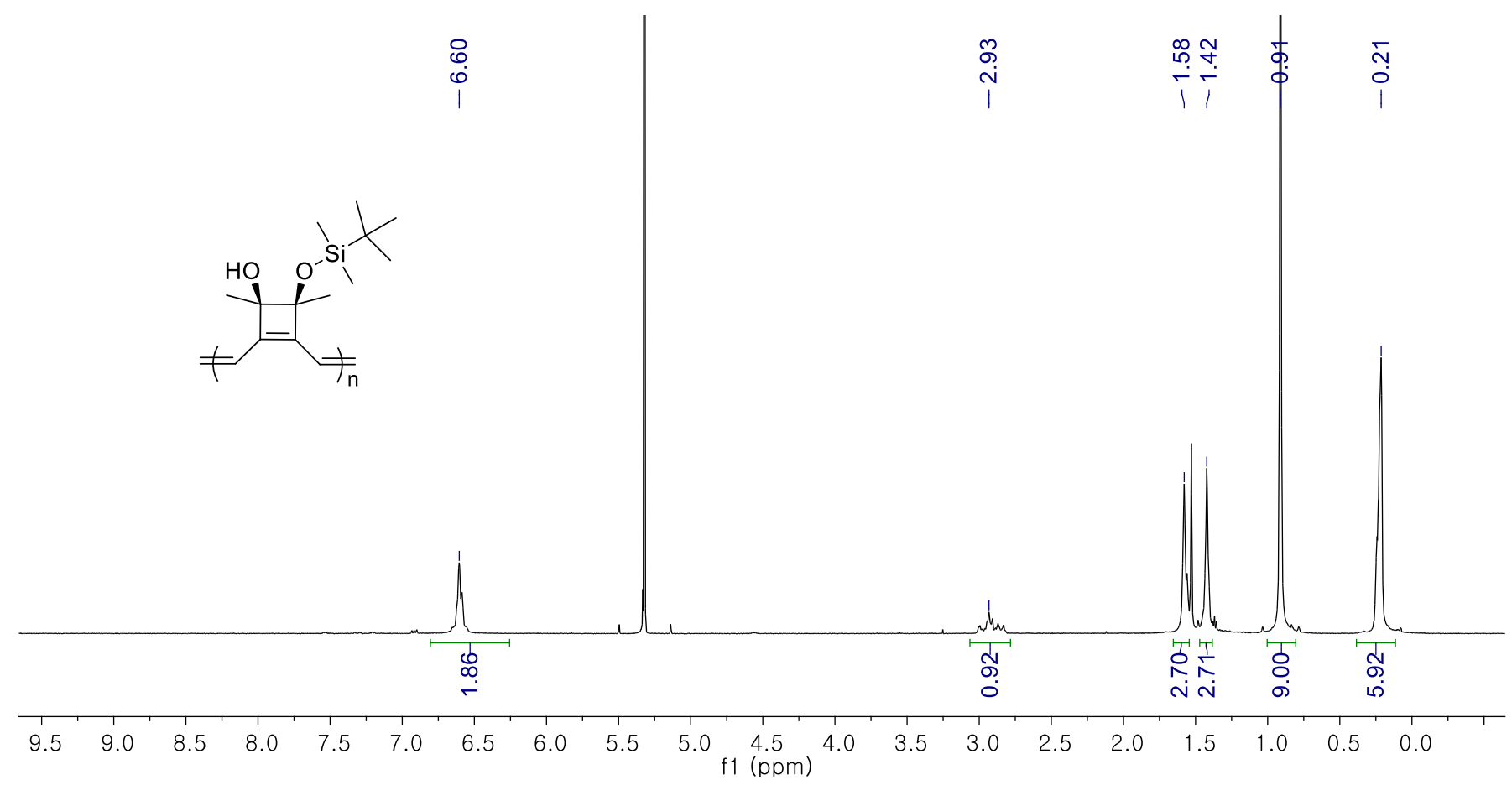

P4 ${ }^{13} \mathrm{C}$ NMR $\left(150 \mathrm{MHz}, \mathrm{CDCl}_{3}\right)$

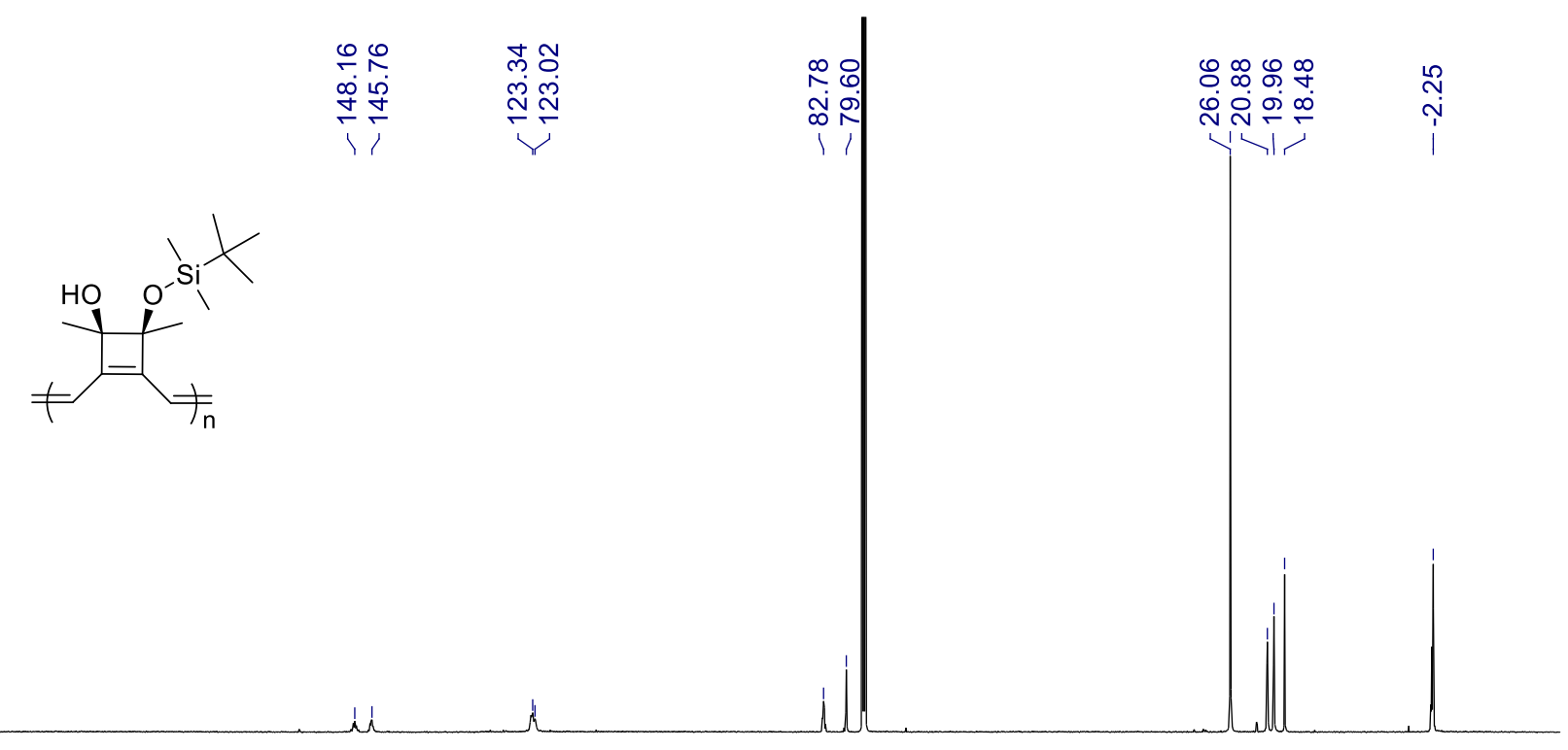

$\begin{array}{llllllllllllllllll}1 & 10 & 170 & 150 & 130 & 110 & 90 & 80 & 70 & 60 & 50 & 40 & 30 & 20 & 10 & 0 & 1\end{array}$


M5 ${ }^{1} \mathrm{H}$ NMR (500 MHz, $\mathrm{CDCl}_{3}$ )

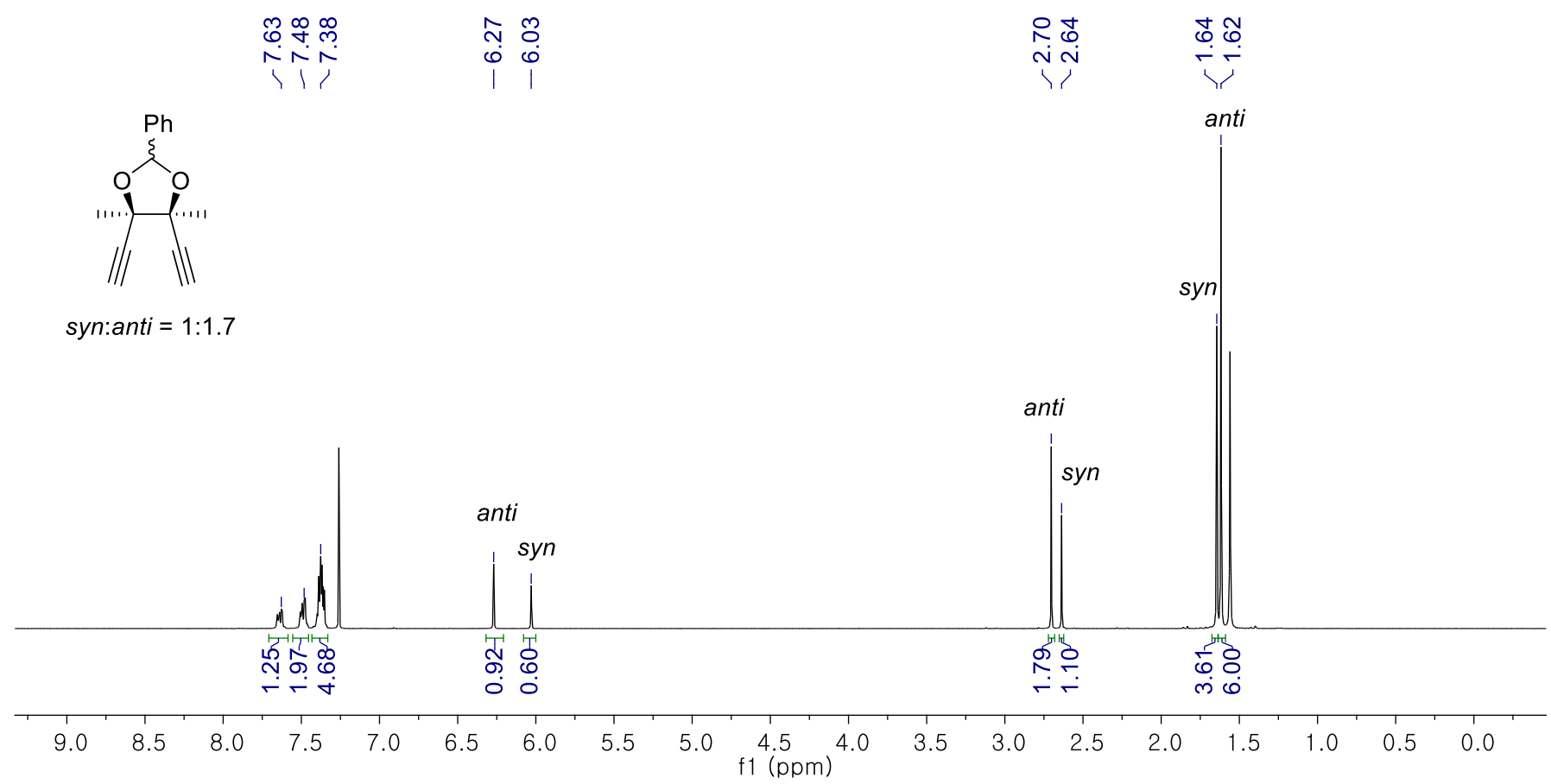

M6 ${ }^{1} \mathrm{H}$ NMR (500 MHz, $\left.\mathrm{CDCl}_{3}\right)$

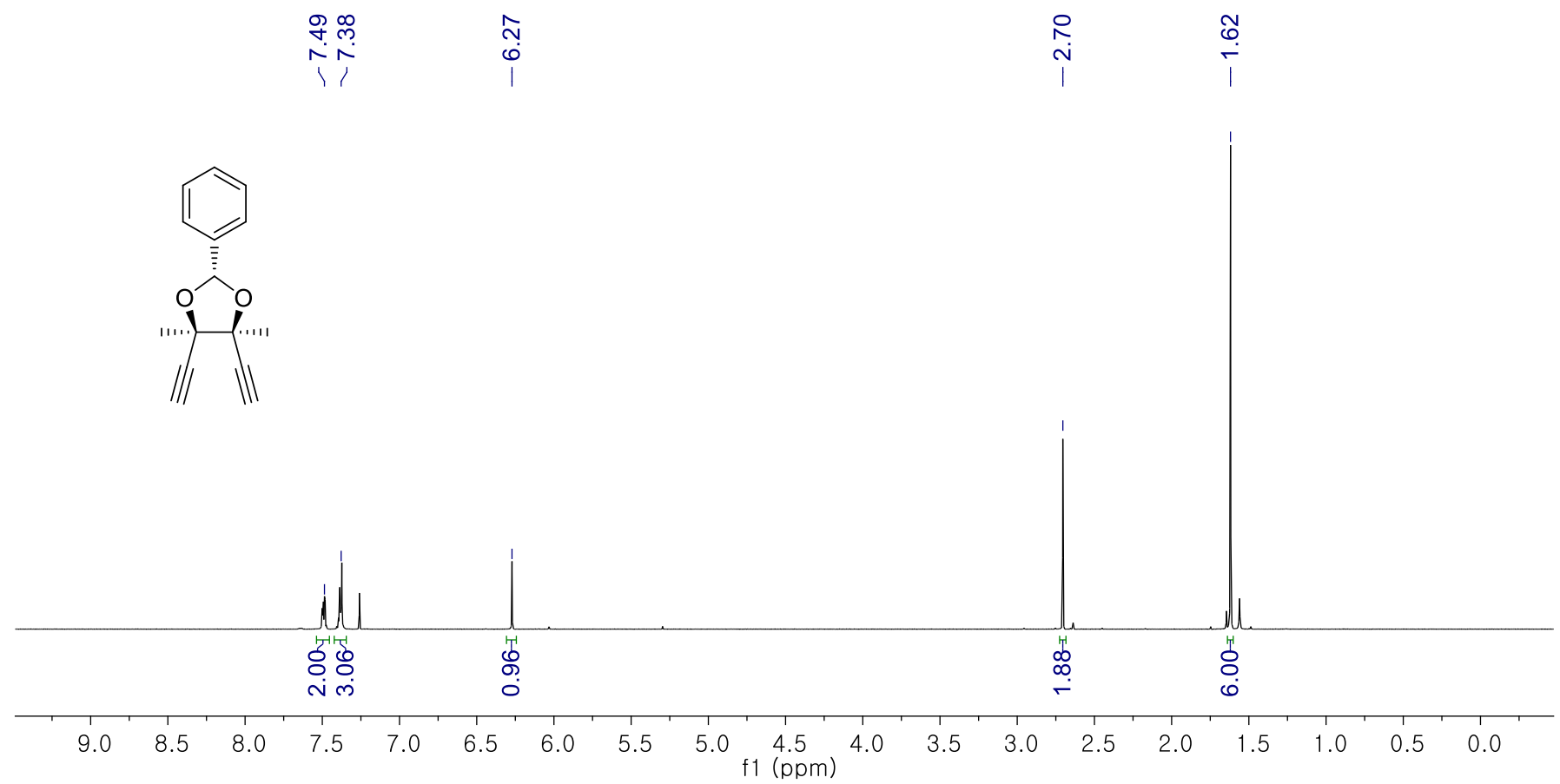


M6 ${ }^{13} \mathrm{C} \mathrm{NMR}\left(125 \mathrm{MHz}, \mathrm{CDCl}_{3}\right)$

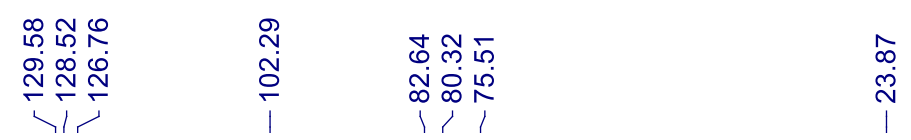<smiles>C#C[C@]1(C)O[C@@H](c2ccccc2)O[C@]1(C)C#C</smiles>

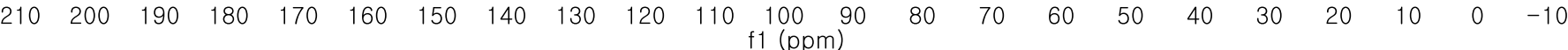


P6 ${ }^{1} \mathrm{H}$ NMR $\left(500 \mathrm{MHz}, \mathrm{CD}_{2} \mathrm{Cl}_{2}\right)$

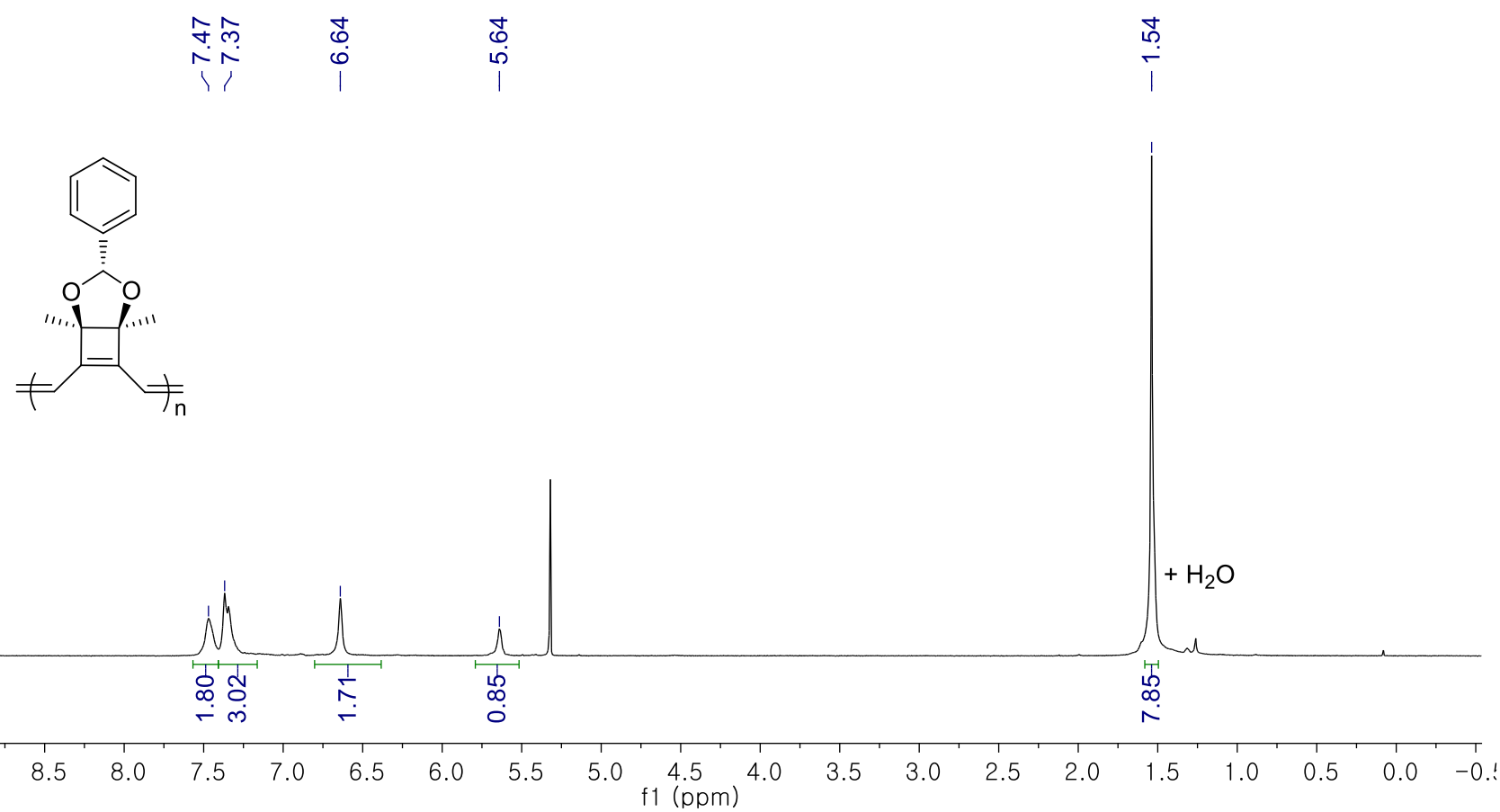

P6 ${ }^{13} \mathrm{C}$ NMR $\left(125 \mathrm{MHz}, \mathrm{CD}_{2} \mathrm{Cl}_{2}\right)$

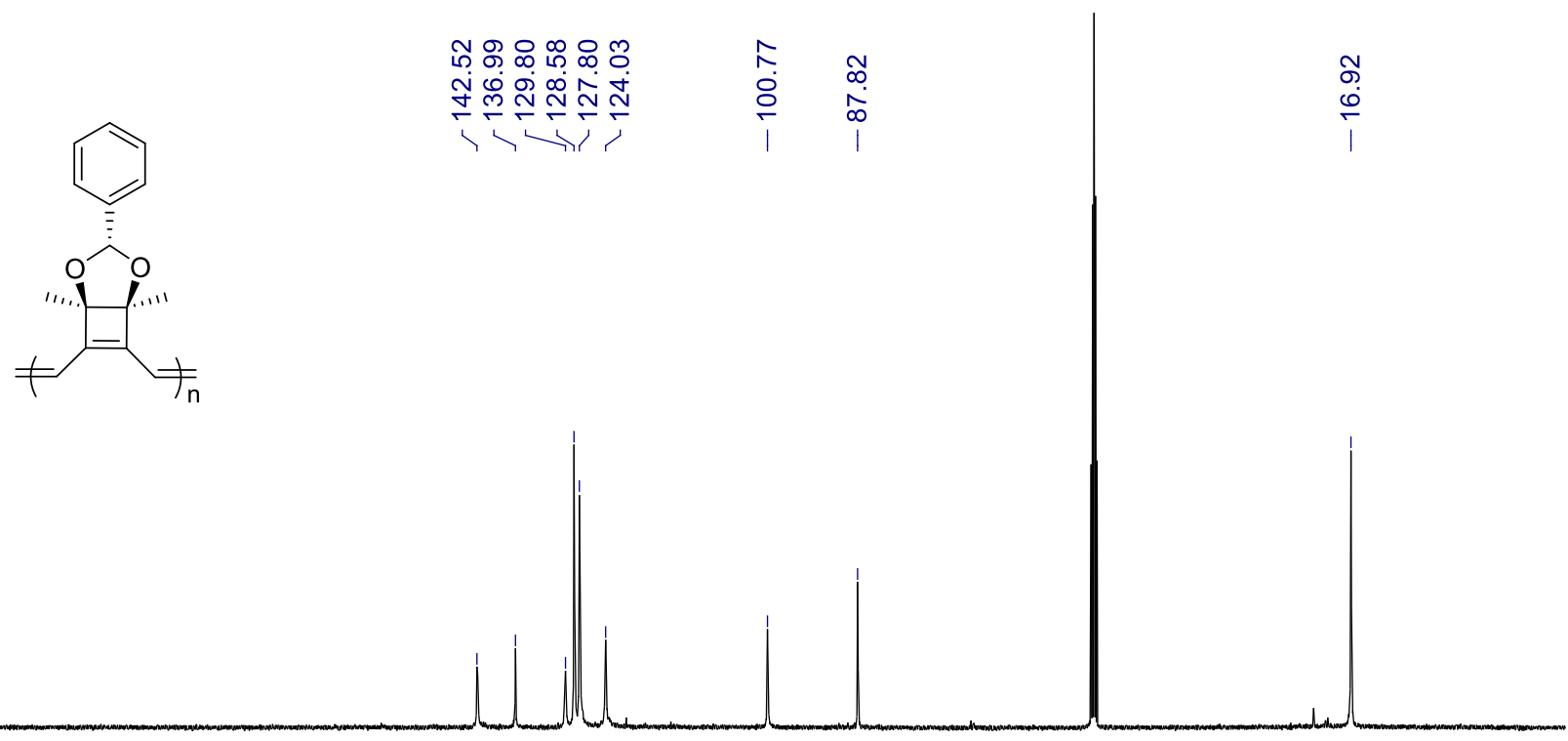

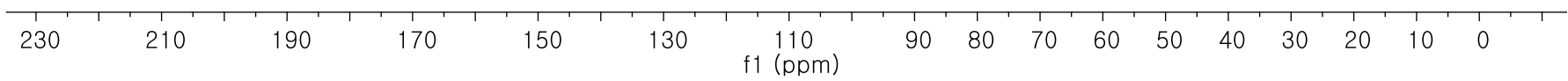




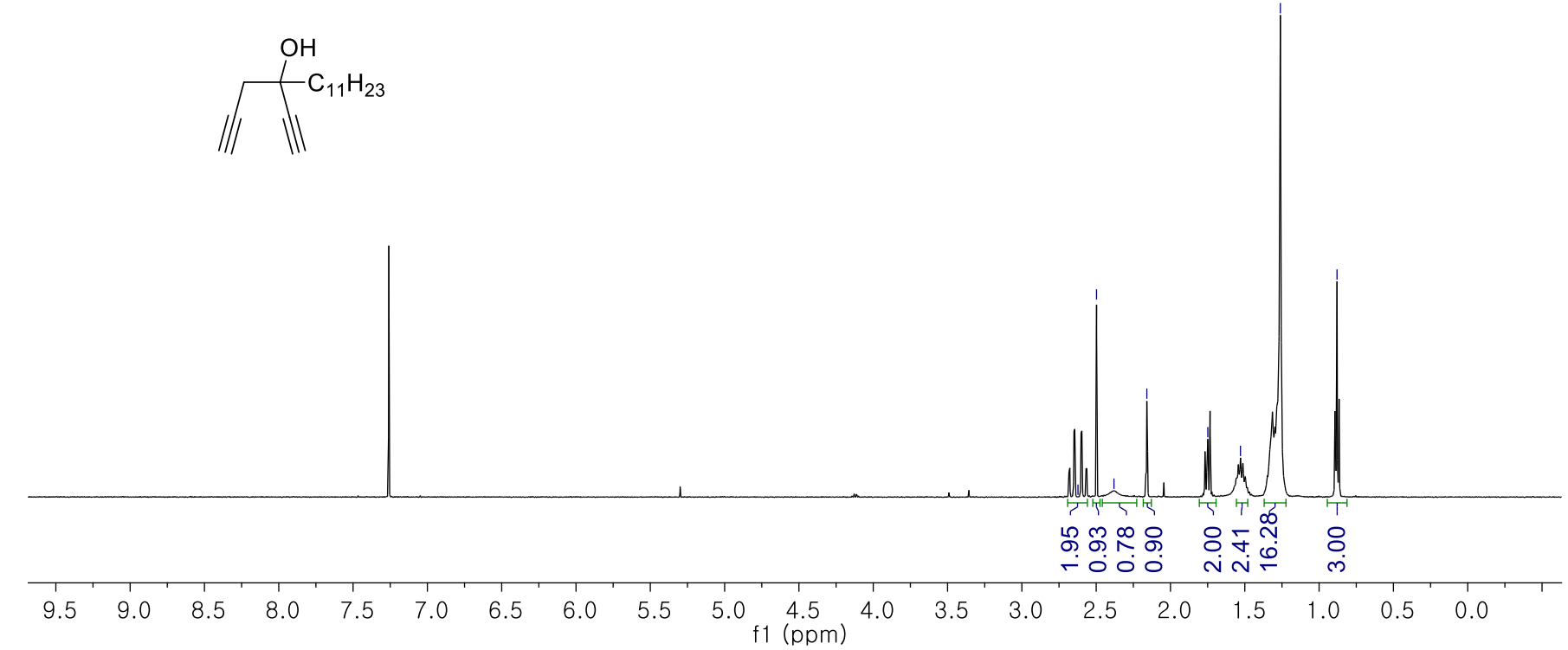

$5{ }^{13} \mathrm{C}$ NMR (125 MHz, $\left.\mathrm{CDCl}_{3}\right)$

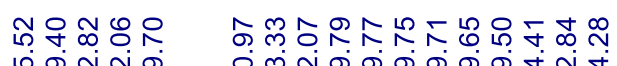

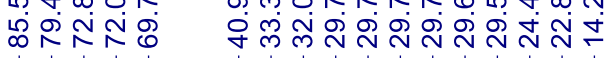
$1<11$<smiles>C#CC(O)(C#C)CC</smiles> 


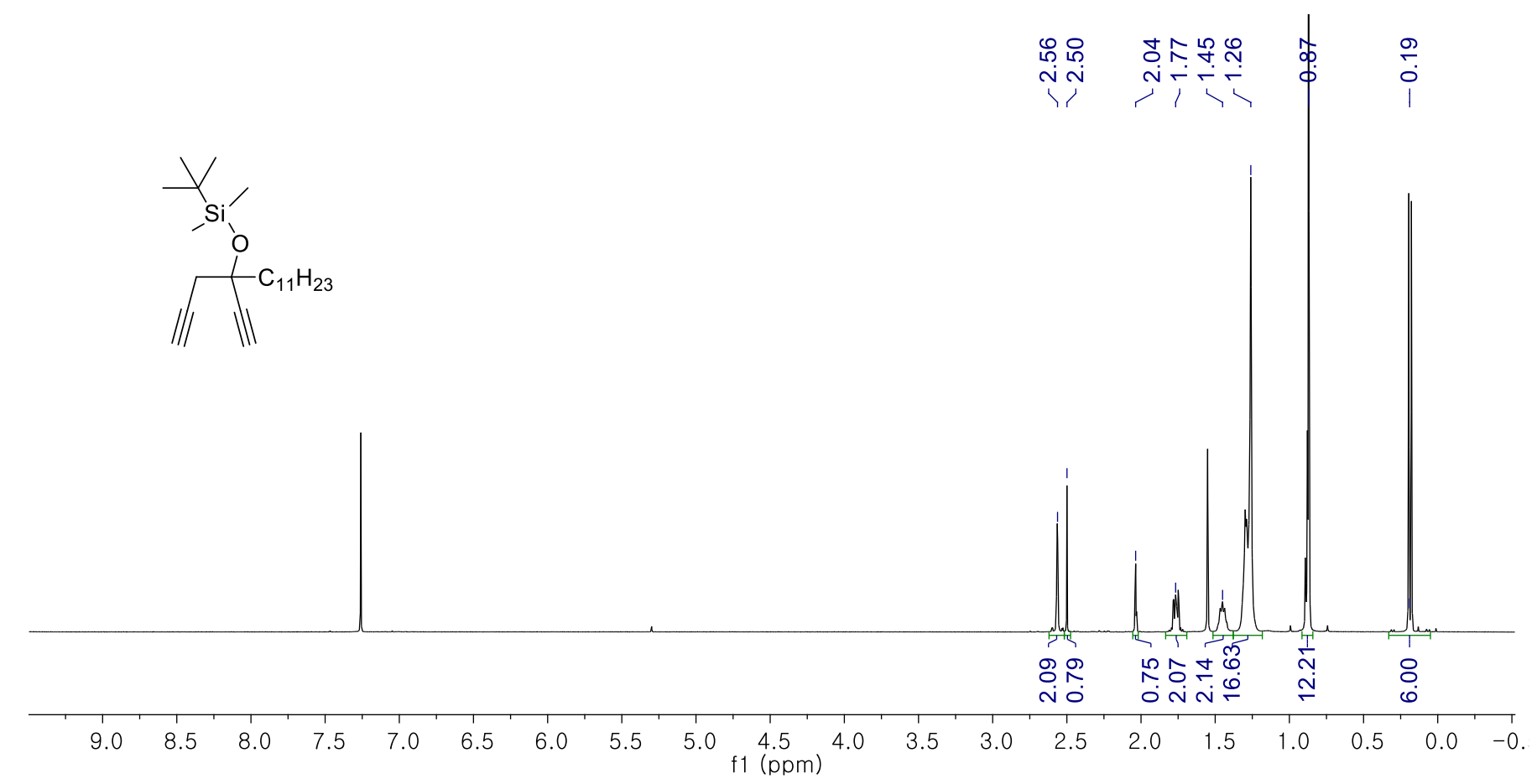

M7 ${ }^{13} \mathrm{C}$ NMR (125 MHz, $\left.\mathrm{CDCl}_{3}\right)$

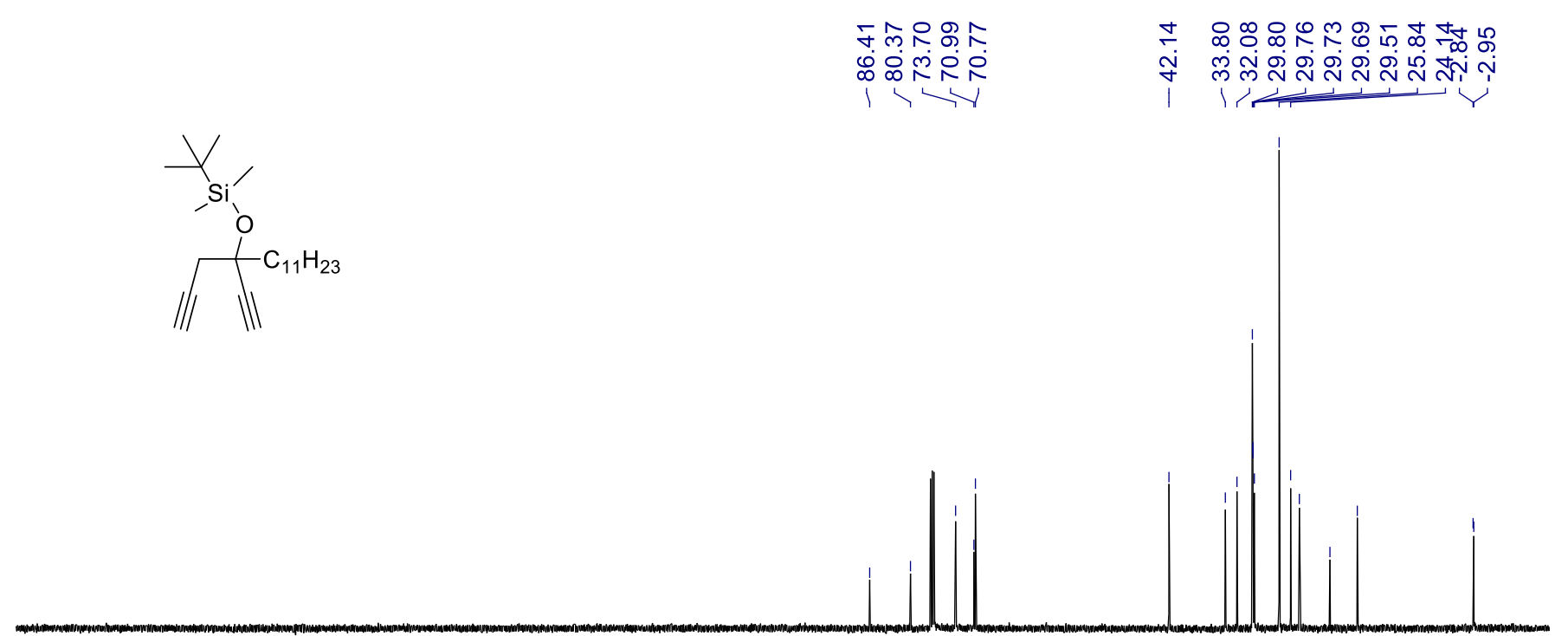

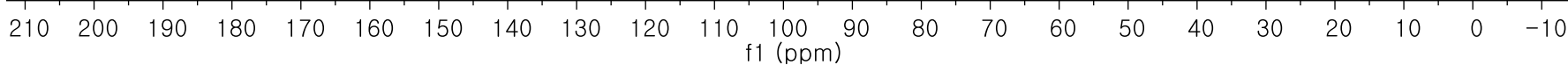


P7 ${ }^{1} \mathrm{H}$ NMR (500 MHz, $\mathrm{CD}_{2} \mathrm{Cl}_{2}$ )

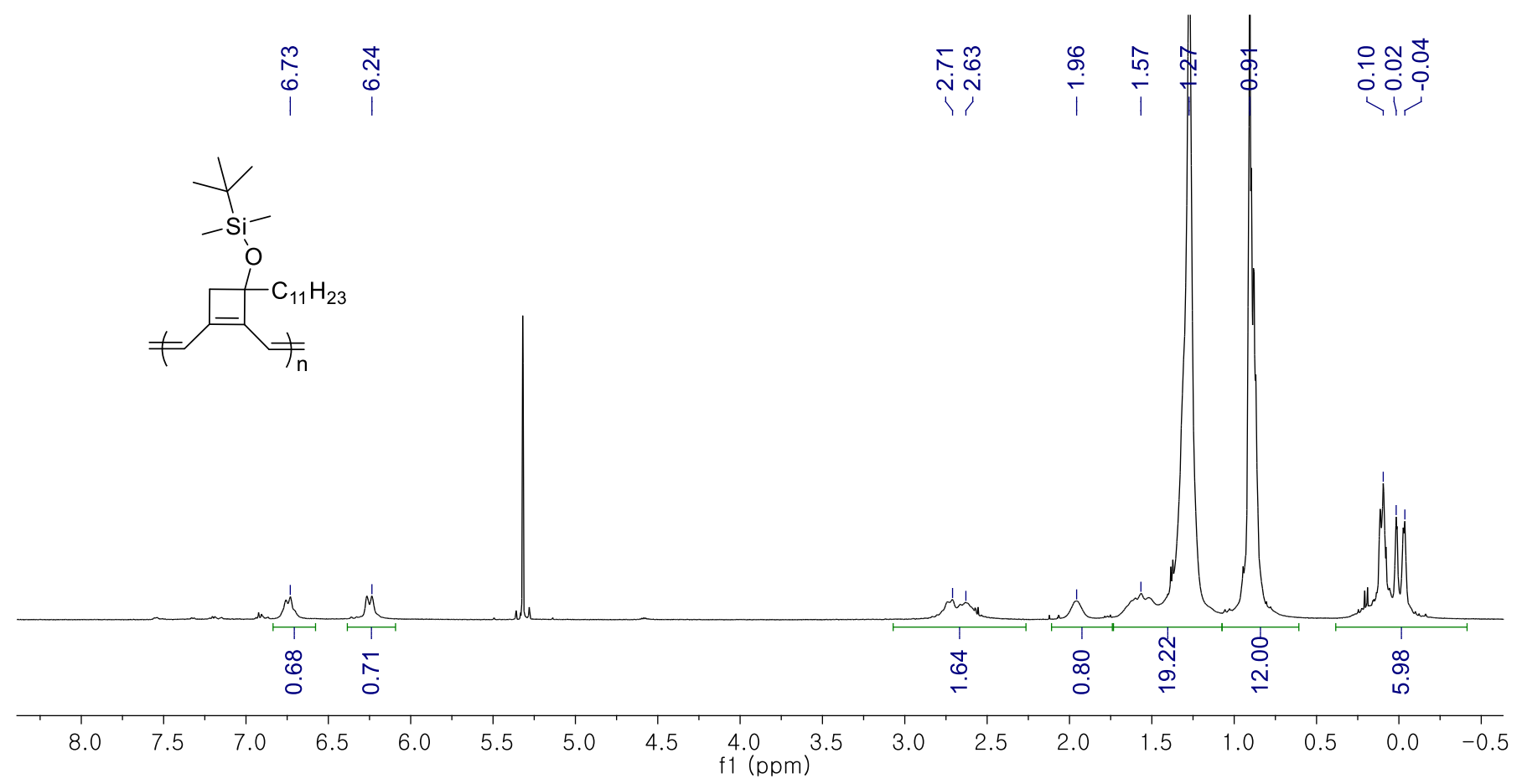

P7 ${ }^{13} \mathrm{C}$ NMR (125 MHz, $\mathrm{CD}_{2} \mathrm{Cl}_{2}$ )

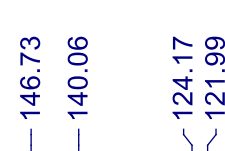

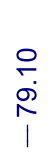

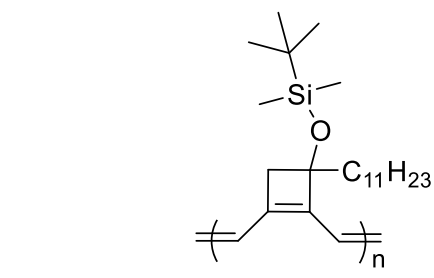

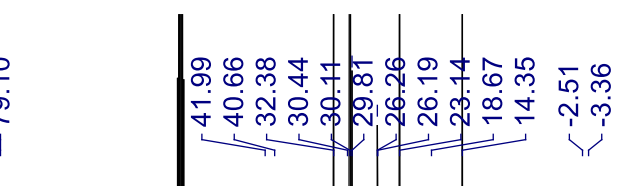

1)

230

210

190

170

150

130

110

$90 \quad 80$

60

$\begin{array}{llllll}50 & 40 & 30 & 20 & 10 & 0\end{array}$ 


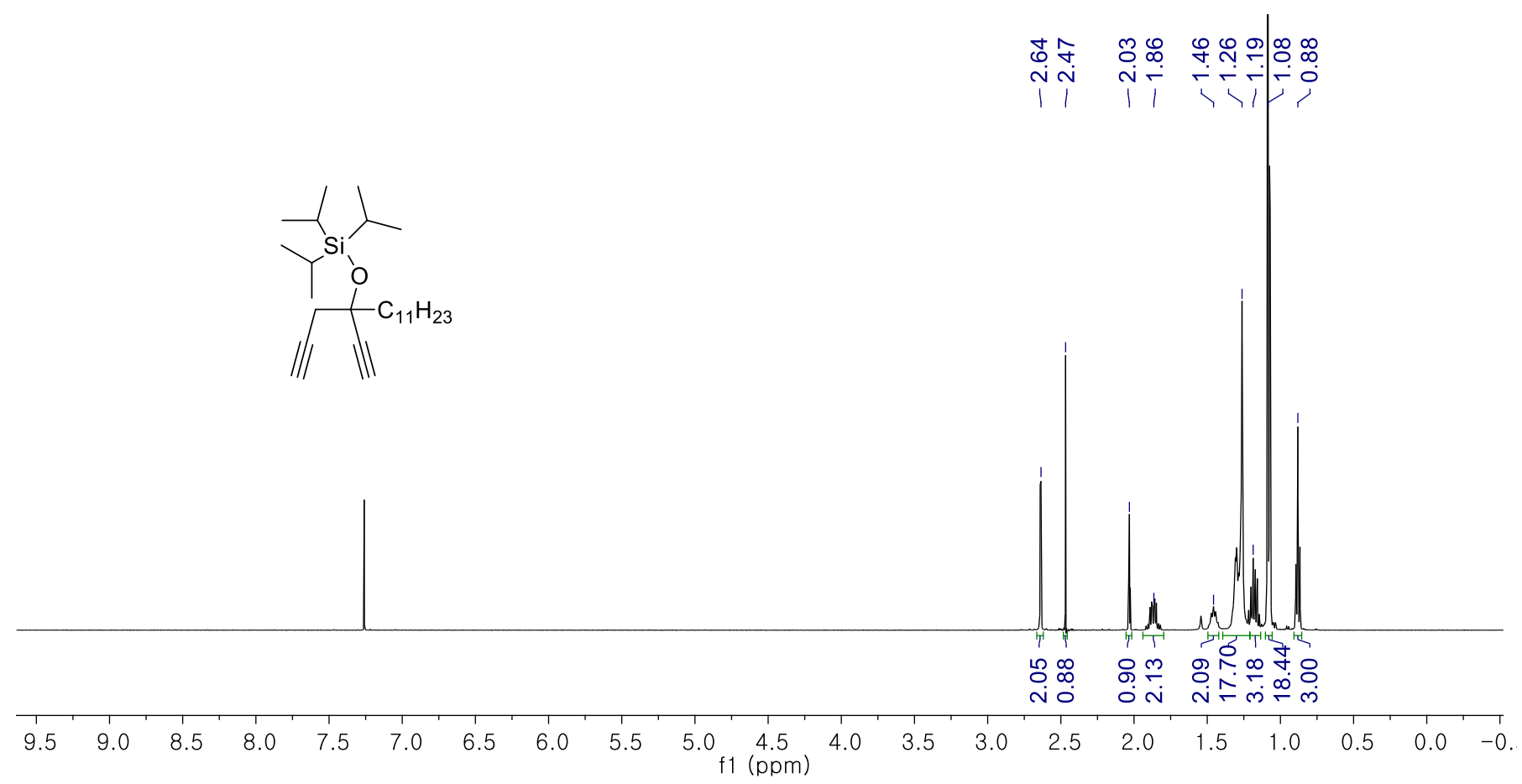

M8 ${ }^{13} \mathrm{C}$ NMR (125 MHz, $\left.\mathrm{CDCl}_{3}\right)$

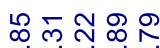

क्षंर요

$1>1$

-

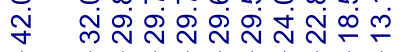

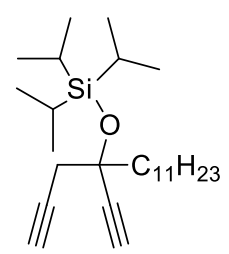

$\begin{array}{llllllllllllllllllllllllllll}210 & 200 & 190 & 180 & 170 & 160 & 150 & 140 & 130 & 120 & \begin{array}{l}110 \\ f 1\end{array} \text { (ppm) } & 100 & 90 & 70 & 60 & 50 & 40 & 30 & 20 & 10 & 0\end{array}$ 
P8 ${ }^{1} \mathrm{H}$ NMR $\left(500 \mathrm{MHz}, \mathrm{CD}_{2} \mathrm{Cl}_{2}\right)$

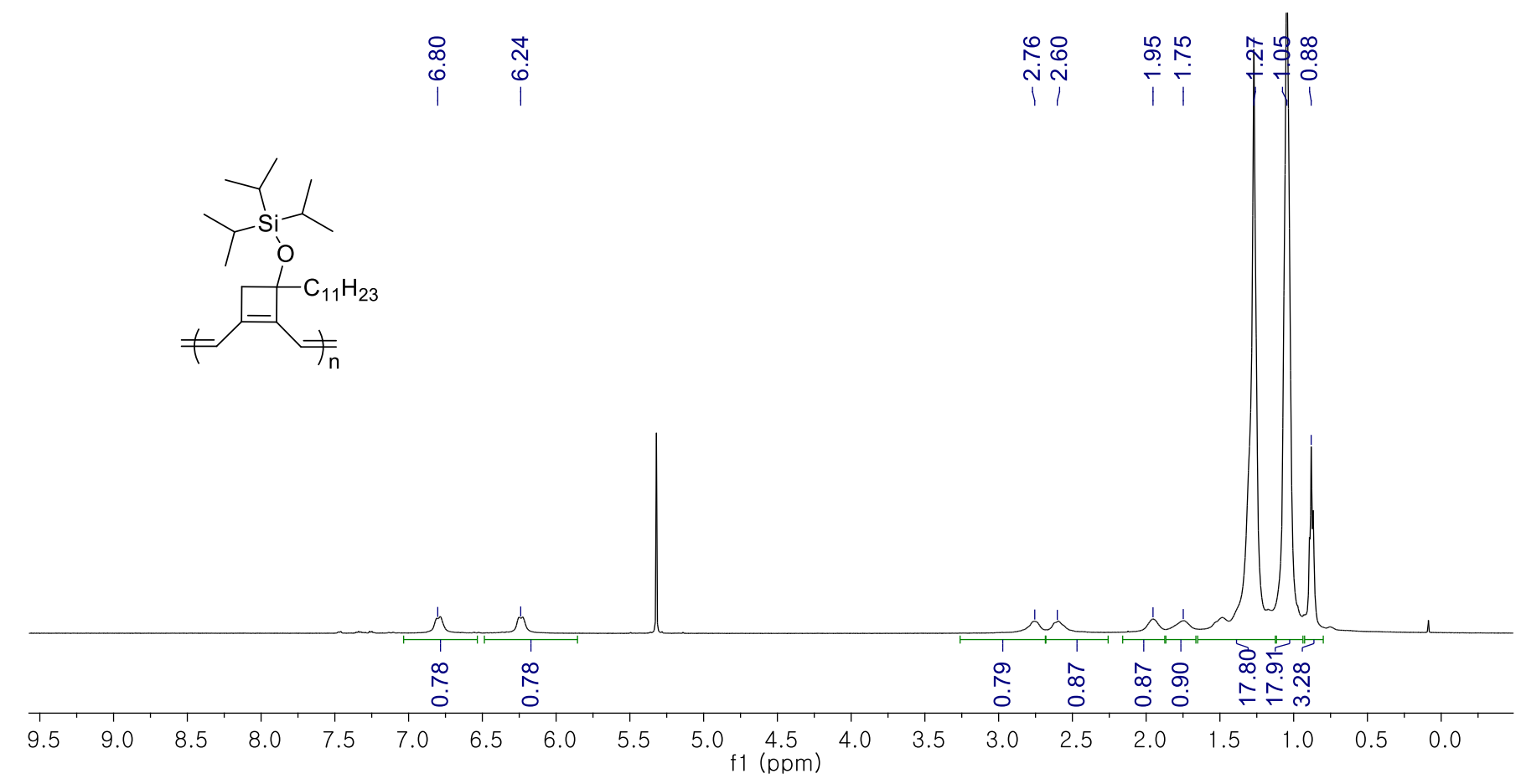

P8 ${ }^{13} \mathrm{C}$ NMR $\left(125 \mathrm{MHz}, \mathrm{CD}_{2} \mathrm{Cl}_{2}\right)$

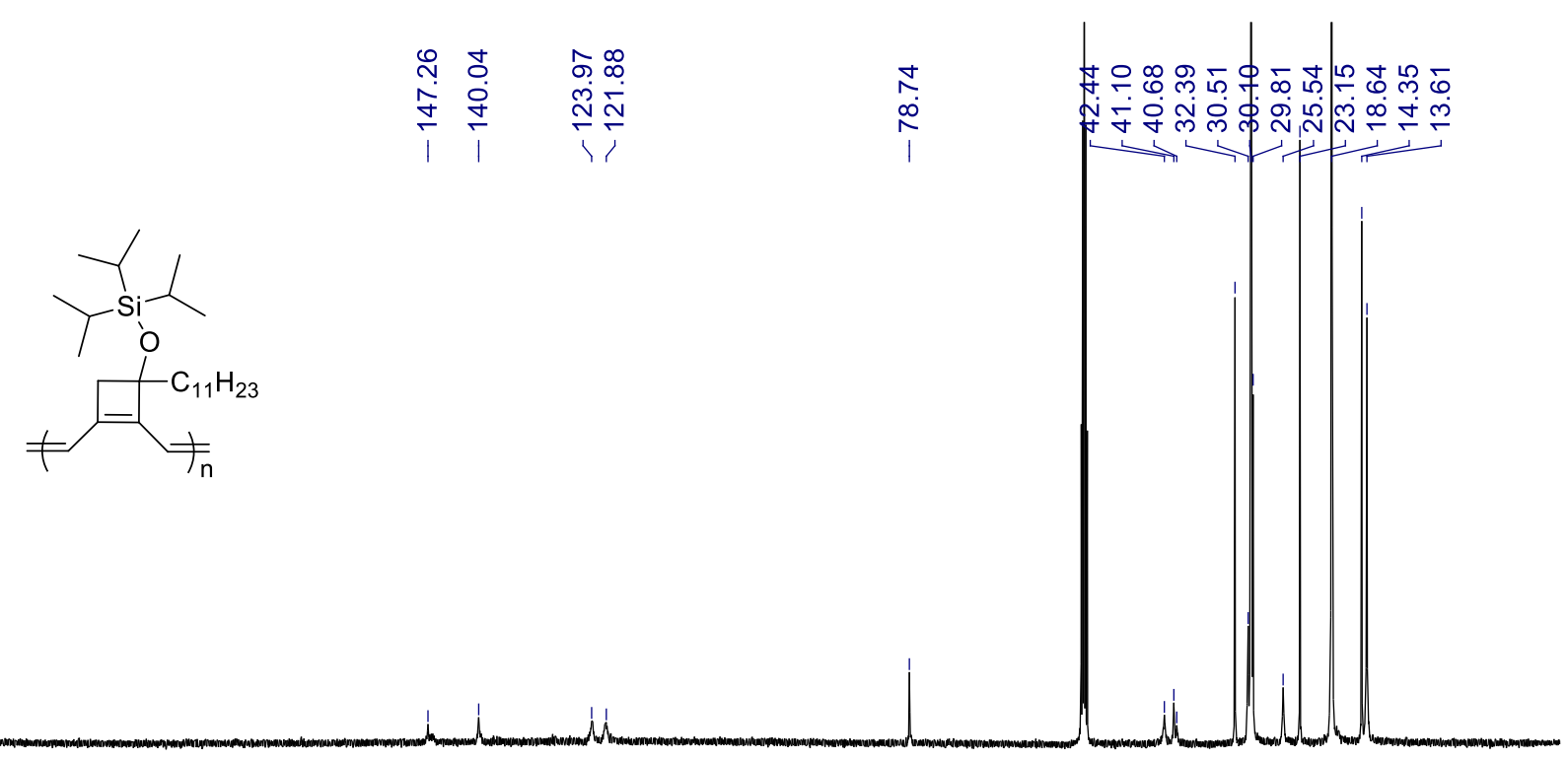

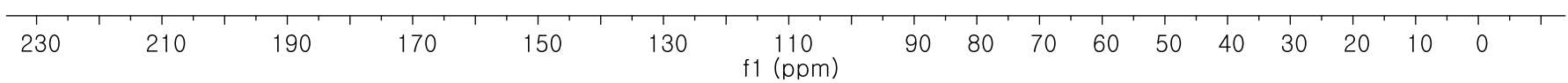


$6{ }^{1} \mathrm{H}$ NMR (500 MHz, $\left.\mathrm{CDCl}_{3}\right)$

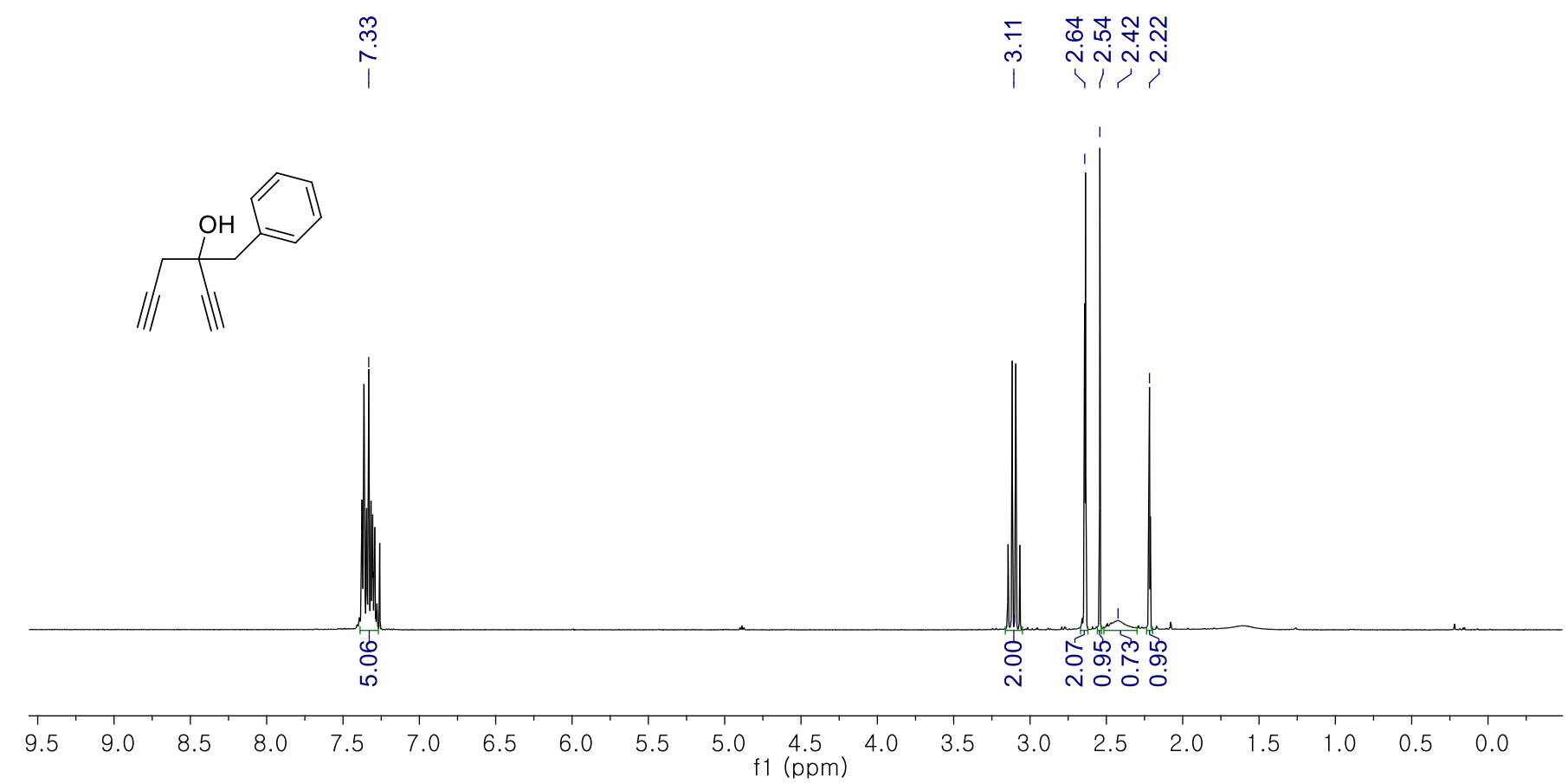

6 ${ }^{13} \mathrm{C}$ NMR (125 MHz, $\left.\mathrm{CDCl}_{3}\right)$

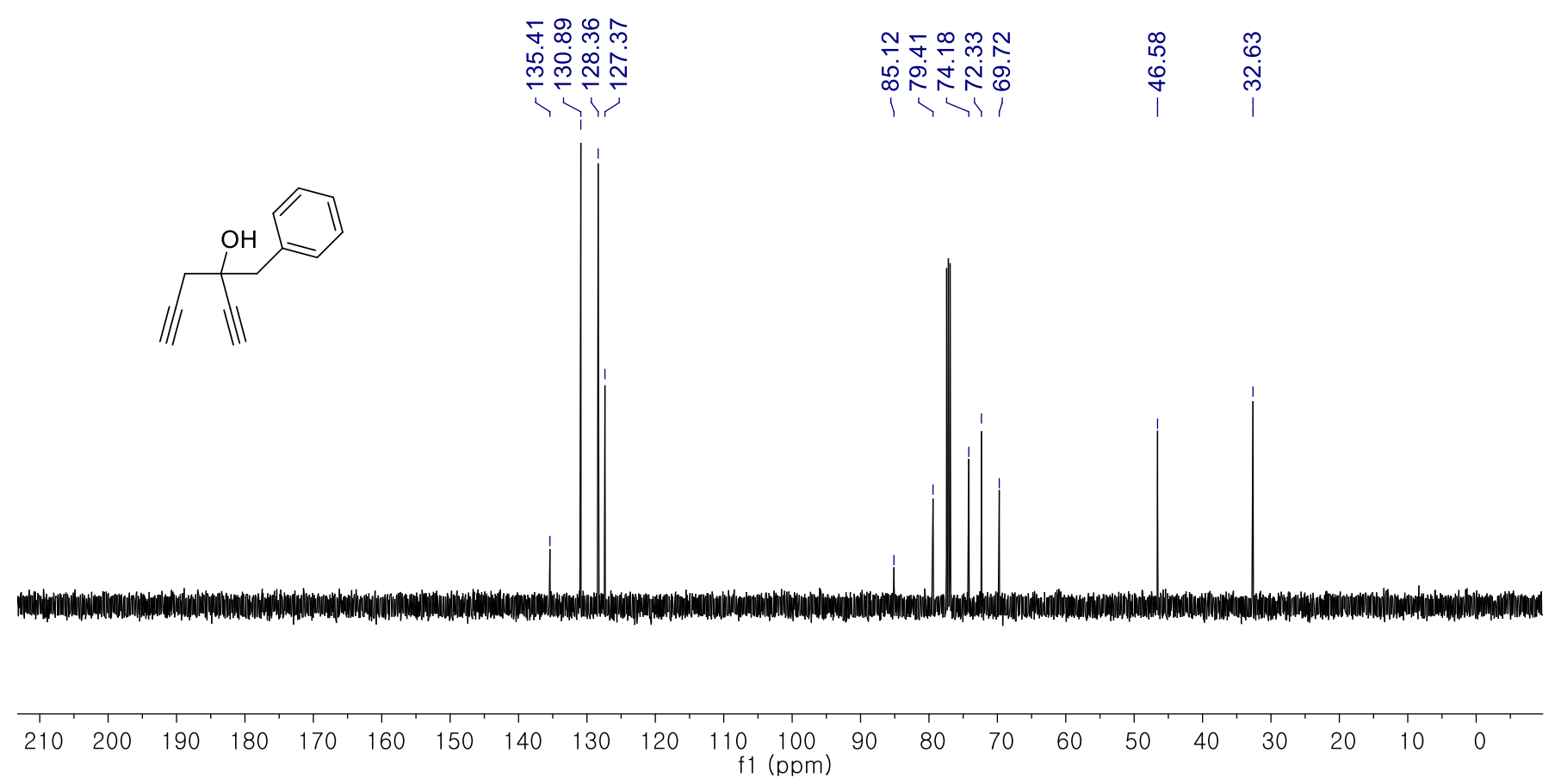




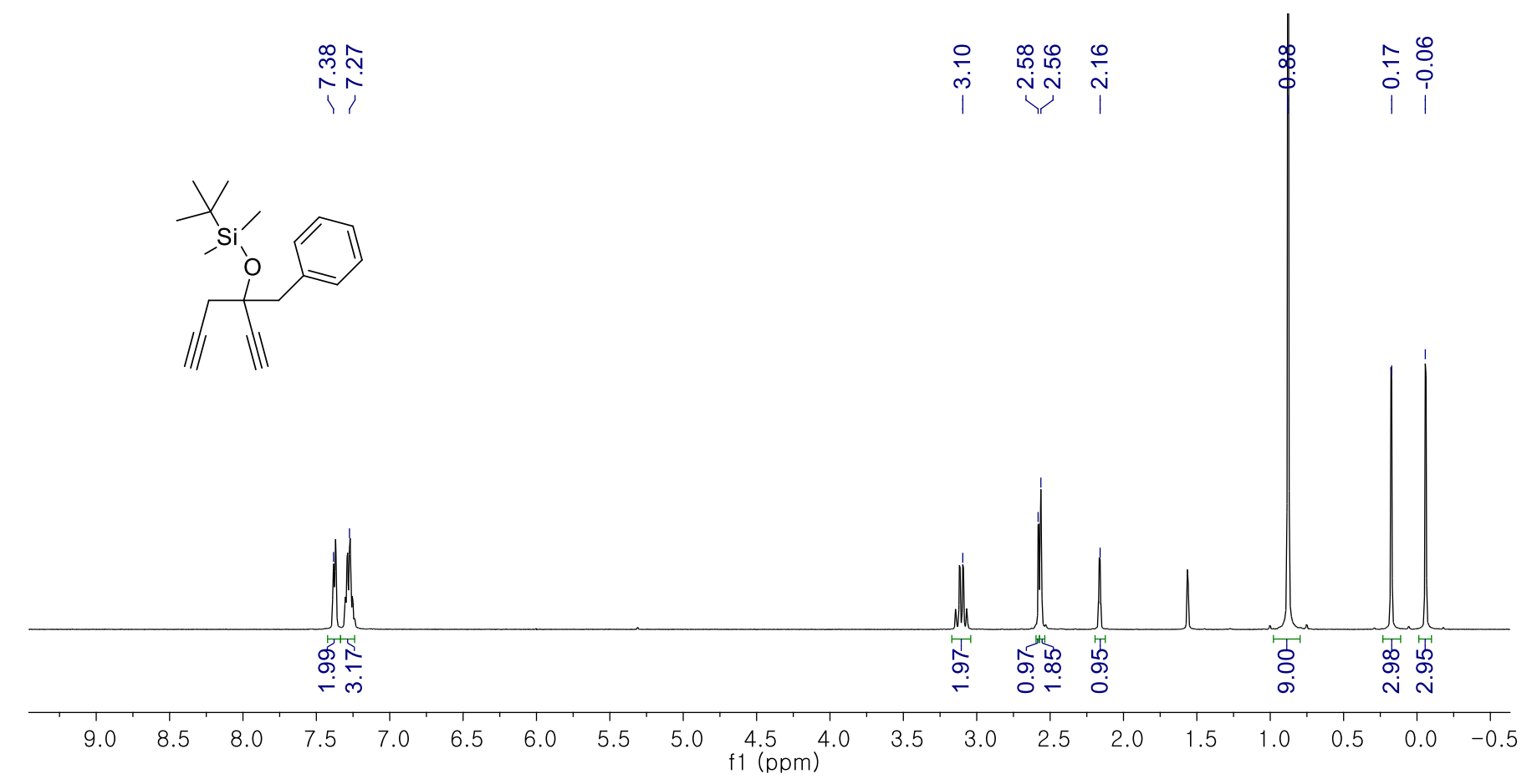

M9 ${ }^{13} \mathrm{C}$ NMR $\left(125 \mathrm{MHz}, \mathrm{CDCl}_{3}\right)$

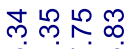

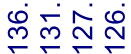

ind

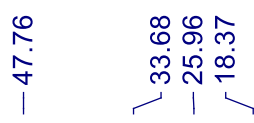

용

กิ
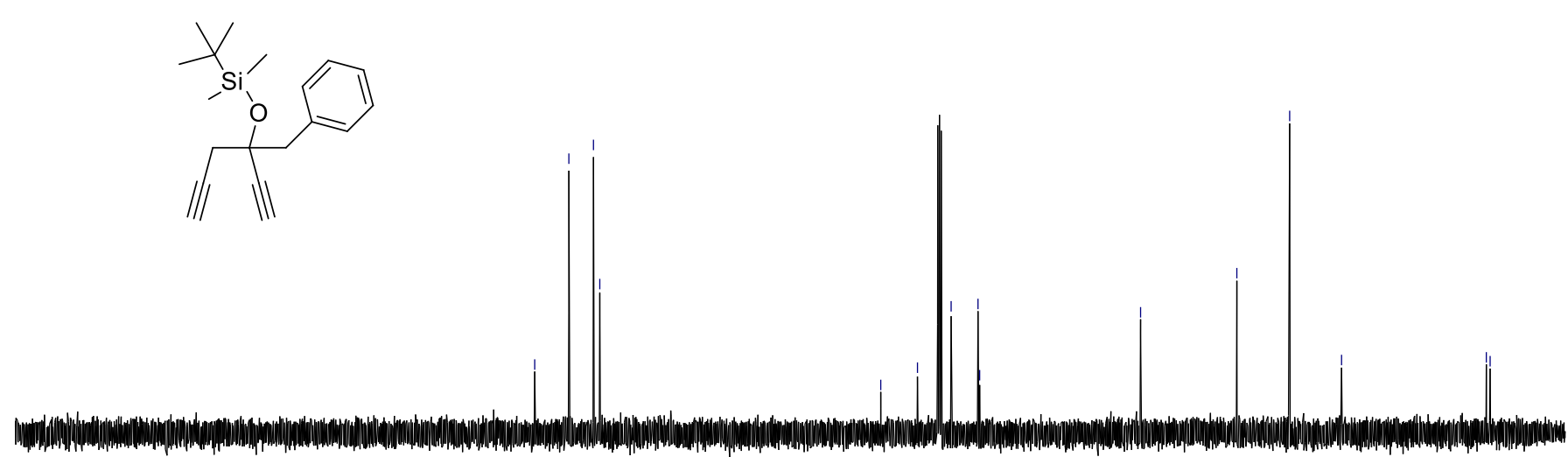

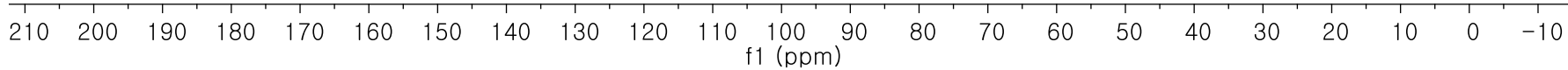


P9 ${ }^{1} \mathrm{H}$ NMR (500 MHz, $\mathrm{CD}_{2} \mathrm{Cl}_{2}$ )

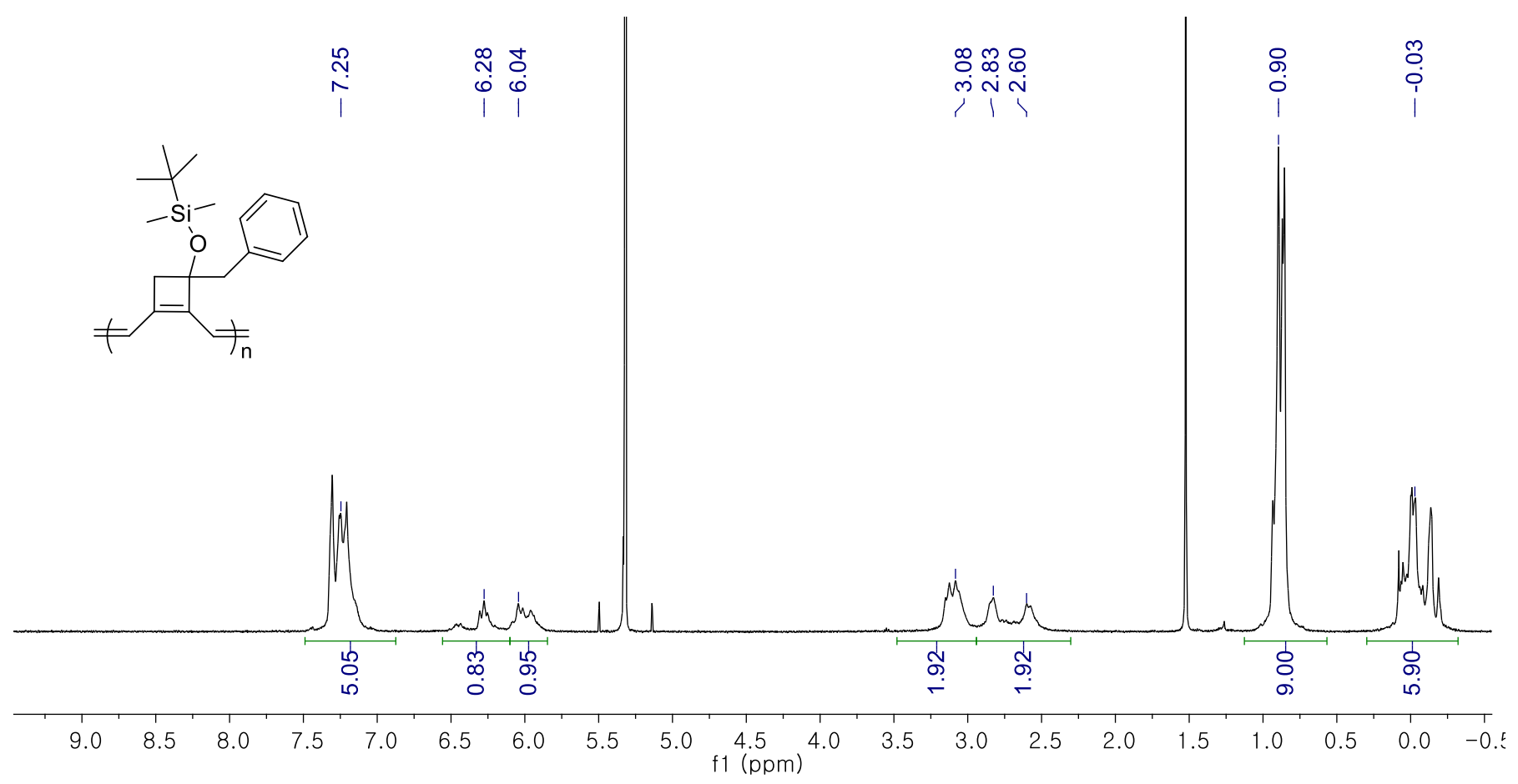

P9 ${ }^{13} \mathrm{C}$ NMR (150 MHz, $\mathrm{CD}_{2} \mathrm{Cl}_{2}$ )

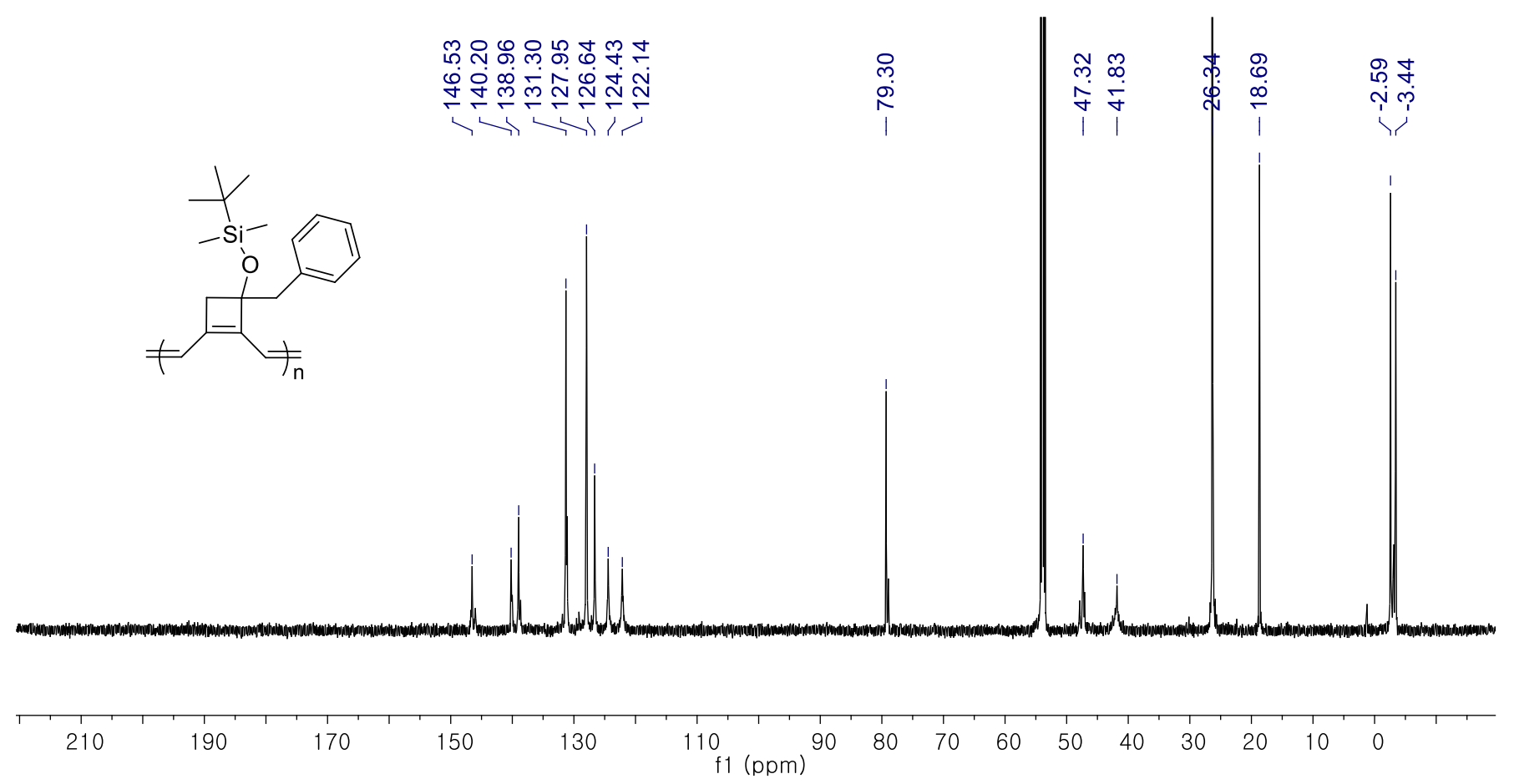




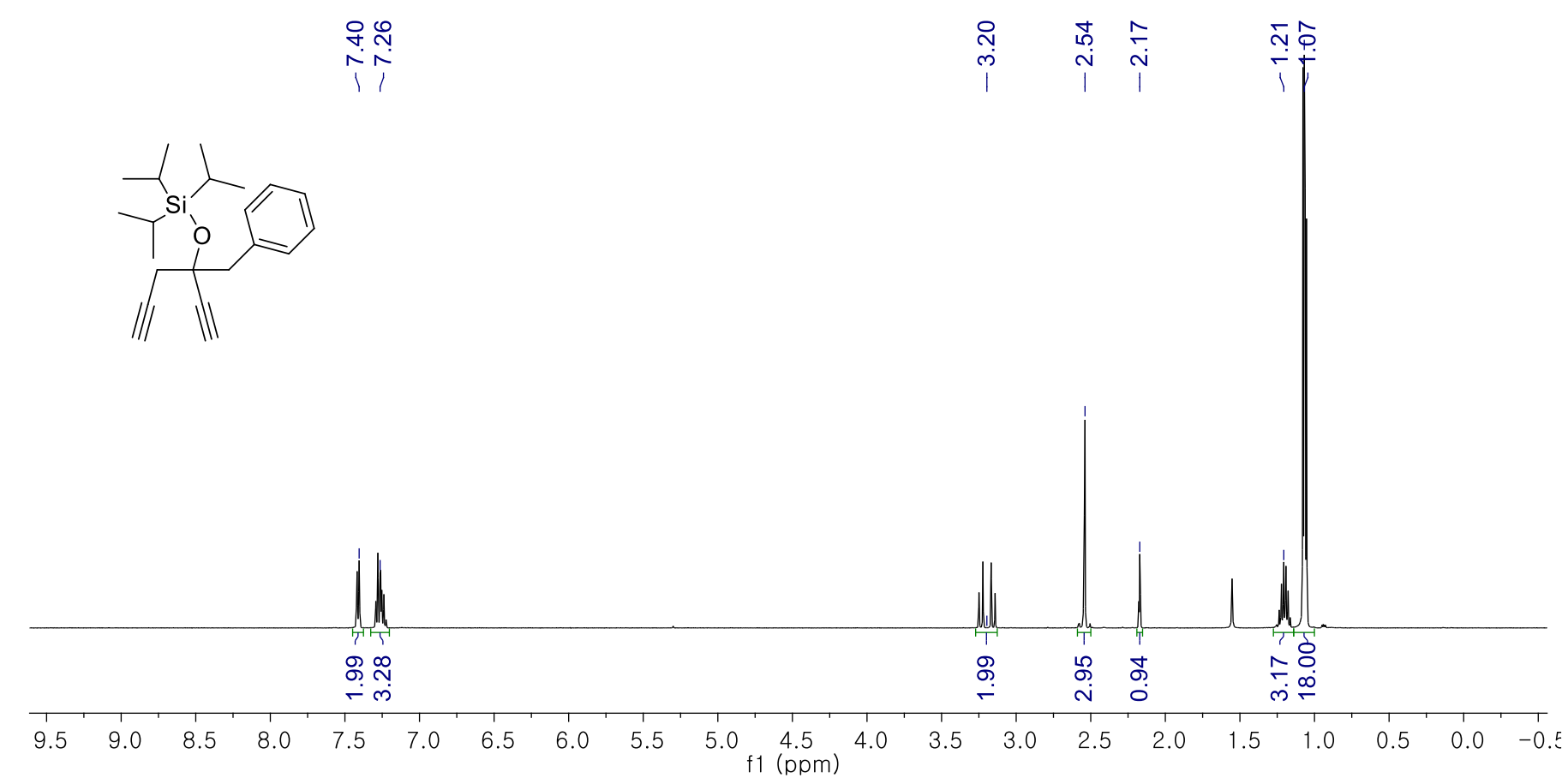

M10 ${ }^{13} \mathrm{C}$ NMR $\left(125 \mathrm{MHz}, \mathrm{CDCl}_{3}\right)$

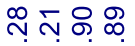

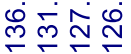

原席

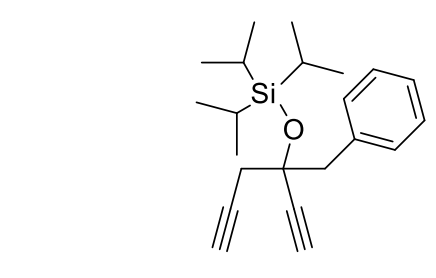

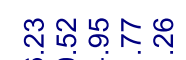

ஹळホス

$1<$ 人।

祍 $\quad$ i

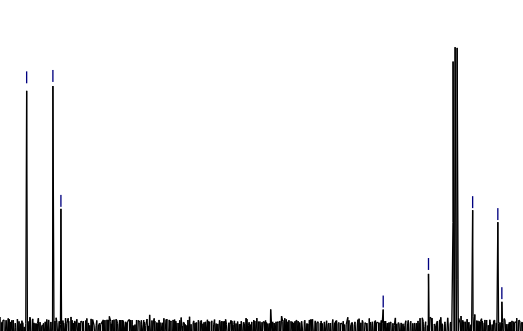

60

40

30

$\begin{array}{lll}20 & 10 & 0\end{array}$ 

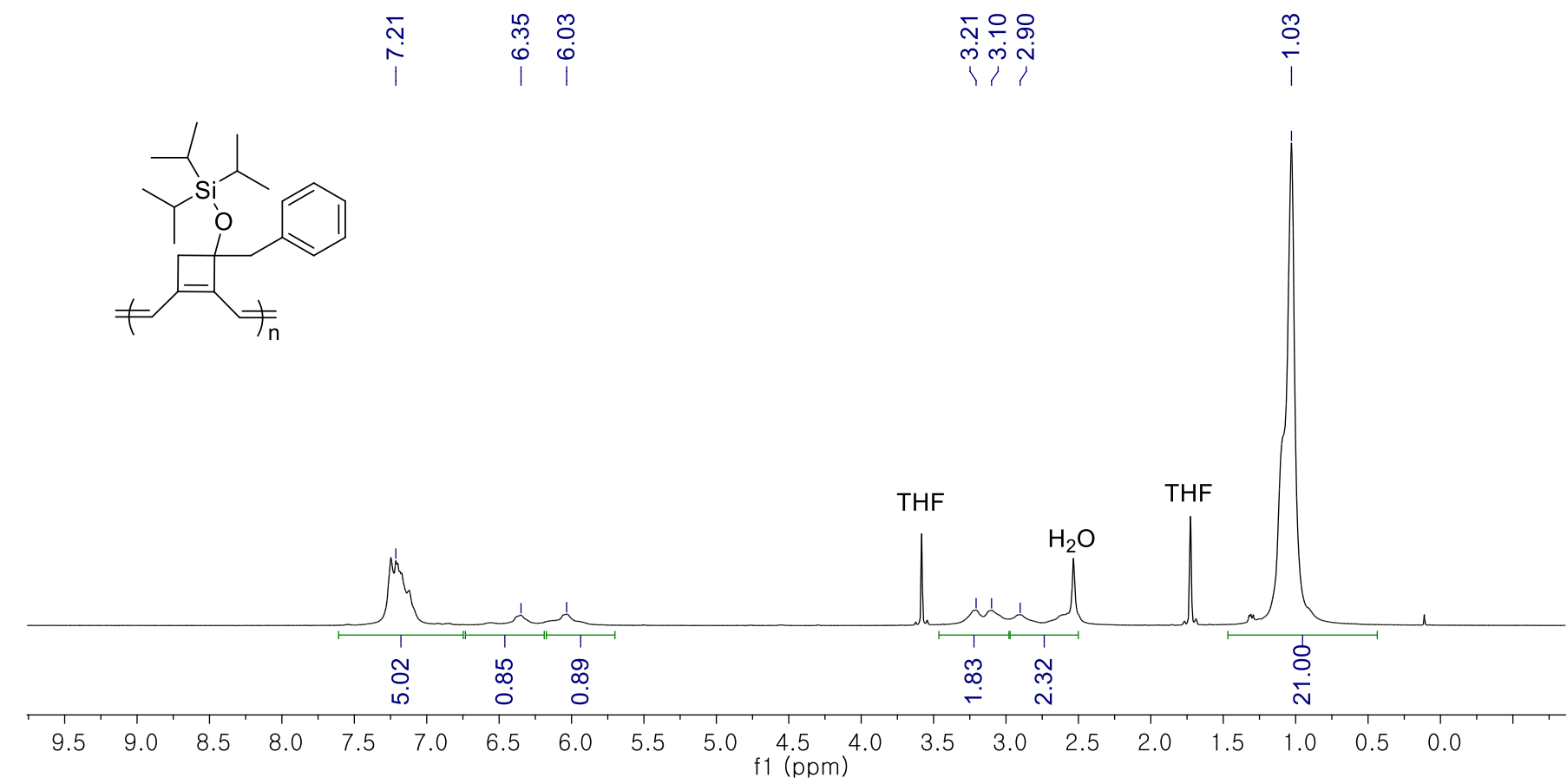

mं

P10 ${ }^{13} \mathrm{C}$ NMR (125 MHz, THF- $\left.d_{8}\right)$

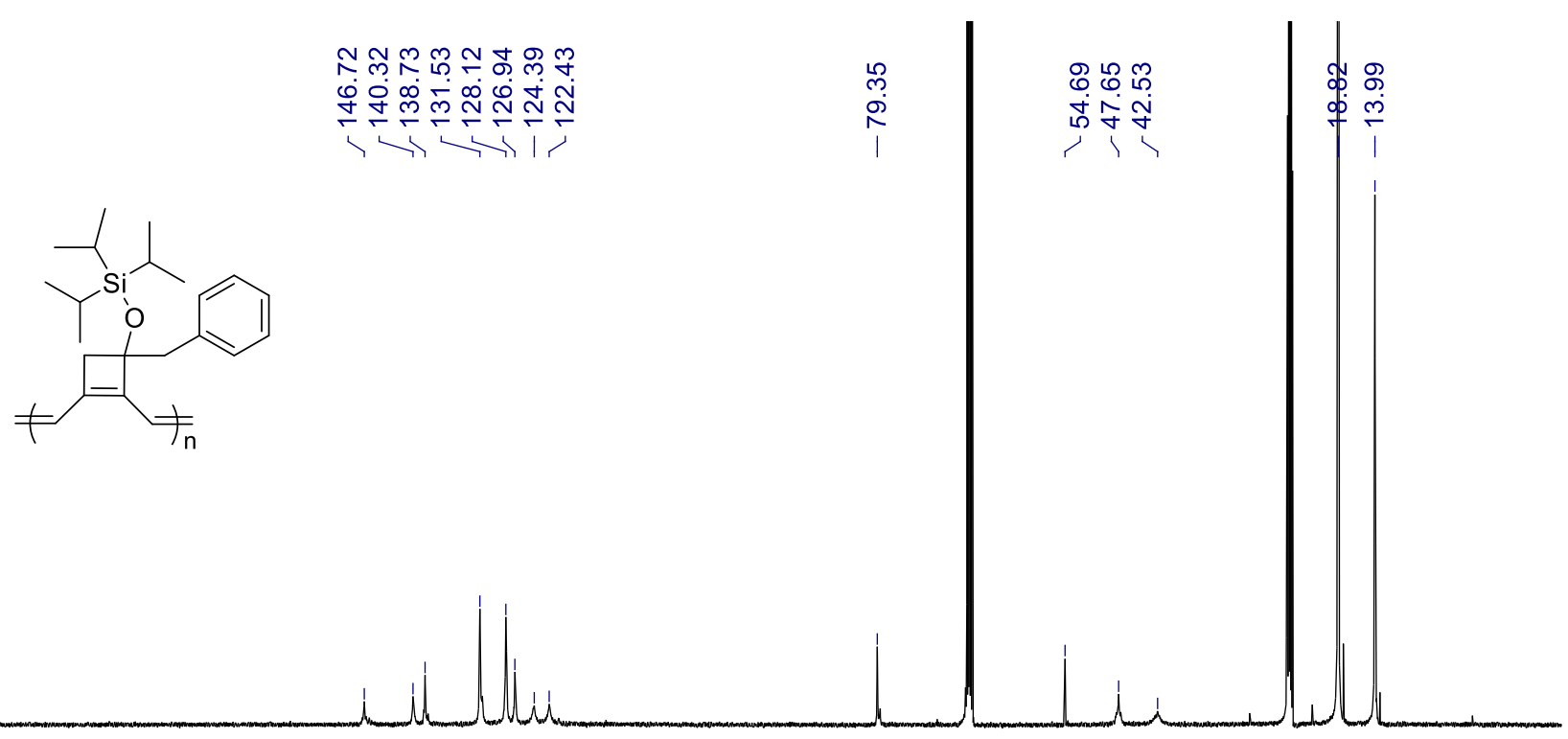

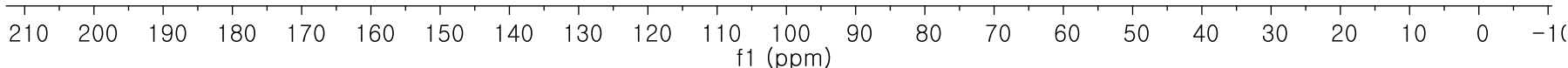


Ru3 ${ }^{1} \mathrm{H}$ NMR $\left(500 \mathrm{MHz}, \mathrm{CD}_{2} \mathrm{Cl}_{2}\right)$

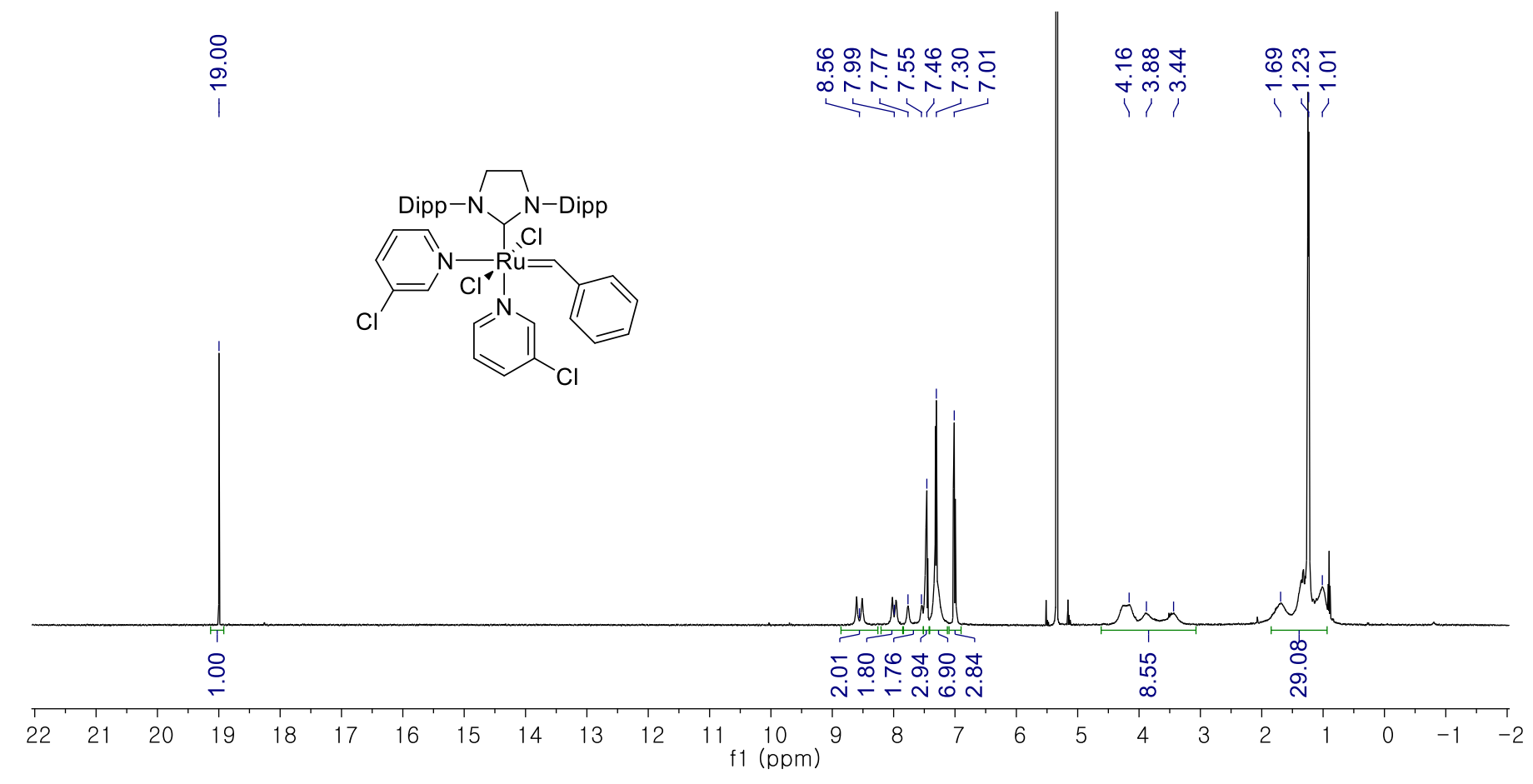

Ru3 ${ }^{13} \mathrm{C}$ NMR $\left(125 \mathrm{MHz}, \mathrm{CD}_{2} \mathrm{Cl}_{2}\right)$

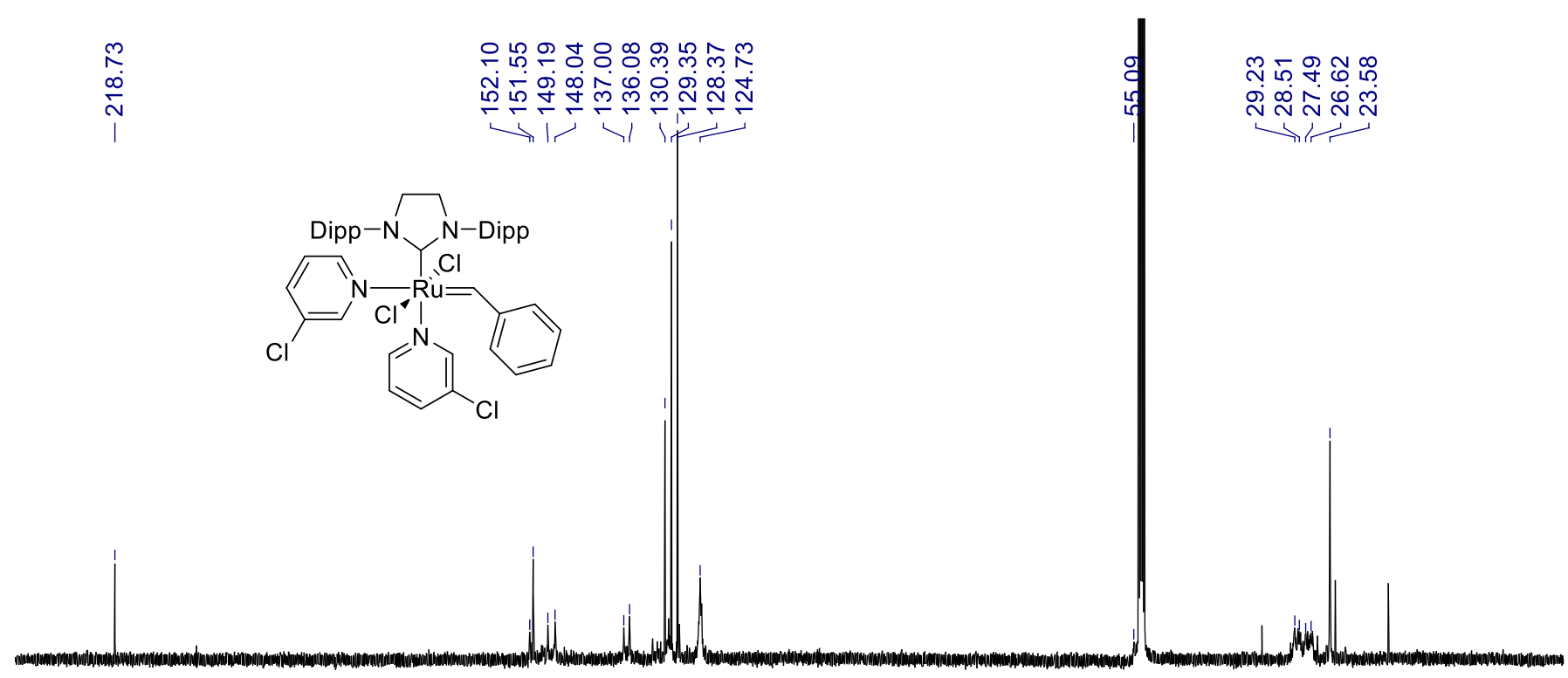

\begin{tabular}{llllllllllllllllll}
\hline 230 & 210 & 190 & 170 & 150 & 130 & $\begin{array}{c}110 \\
f 1(\mathrm{ppm})\end{array}$ & 90 & 80 & 70 & 60 & 50 & 40 & 30 & 20 & 10 & 0 & 1
\end{tabular}




\section{References}

(1) Leitao, E. M.; Piers, W. E.; Parvez, M. A thermally robust ruthenium phosphonium alkylidene catalyst - the effect of more bulky $N$-heterocyclic carbene ligands on catalyst performance in olefin metathesis reactions. Can. J. Chem. 2013, 91, 935-942.

(2) Frisch, M. J.; Trucks, G. W.; Schlegel, H. B.; Scuseria, G. E.; Robb, M. A.; Cheeseman, J. R.; Scalmani, G.; Barone, V.; Mennucci, B.; Petersson, G. A. Gaussian 09, revision A.02; Gaussian, Inc.: Wallingford, CT, 2009.

(3) Alabugin, I. V.; Timokhin, V. I.; Abrams, J. N.; Manoharan, M.; Abrams, R.; Ghiviriga, I. In search of efficient 5-endo-dig cyclization of a carbon-centered radical: 40 Years from a prediction to another success for the Baldwin rules. J. Am. Chem. Soc. 2008, 130, 10984-10995.

(4) Shiina, I.; Umezaki, Y.; Kuroda, N.; Iizumi, T.; Nagai, S.; Katoh, T. MNBA-mediated $\beta$-lactone formation: mechanistic studies and application for the asymmetric total synthesis of tetrahydrolipstatin. J. Org. Chem. 2012, 77, 4885-4901.

(5) López, F.; Castedo, L.; Mascareñas, J. L. Atom-Efficient Assembly of 1, 5-Oxygen-Bridged Medium-Sized Carbocycles by Sequential Combination of a Ru-Catalyzed Alkyne-Alkene Coupling and a Prins-Type Cyclization. J. Am. Chem. Soc. 2002, 124, 4218-4219. 


$\begin{array}{rrrr}\mathbf{A}(\mathrm{DP}=1) & & & \\ \mathrm{C} & 1.690129 & -0.984768 & -0.000259 \\ \mathrm{H} & 1.333402 & -2.014964 & 0.000065 \\ \mathrm{C} & 0.682705 & 0.049584 & -0.000022 \\ \mathrm{C} & 0.784364 & 1.56672 & -0.000076 \\ \mathrm{C} & -0.784364 & 1.56672 & 0.000076 \\ \mathrm{H} & 1.246001 & 2.011905 & -0.890099 \\ \mathrm{H} & 1.246129 & 2.011951 & 0.889816 \\ \mathrm{H} & -1.246129 & 2.011951 & -0.889816 \\ \mathrm{H} & -1.246001 & 2.011905 & 0.890099 \\ \mathrm{C} & -0.682705 & 0.049584 & 0.000022 \\ \mathrm{C} & -1.690129 & -0.984768 & 0.000259 \\ \mathrm{H} & -1.333402 & -2.014964 & -0.000065 \\ \mathrm{C} & -3.013173 & -0.75001 & -0.000033 \\ \mathrm{H} & -3.410958 & 0.261802 & -0.00034 \\ \mathrm{H} & -3.736269 & -1.55985 & -0.000391 \\ \mathrm{C} & 3.013173 & -0.75001 & 0.000033 \\ \mathrm{H} & 3.736269 & -1.55985 & 0.000391 \\ \mathrm{H} & 3.410958 & 0.261802 & 0.00034\end{array}$

A $(\mathrm{DP}=3)$

$\begin{array}{lrrr}\mathrm{C} & -0.000027 & 7.725832 & 0.230463 \\ \mathrm{H} & -0.000021 & 8.12148 & -0.782137 \\ \mathrm{C} & -0.000012 & 6.401755 & 0.468367 \\ \mathrm{H} & -0.00002 & 6.048626 & 1.499865 \\ \mathrm{C} & 0.000012 & 5.39294 & -0.55984 \\ \mathrm{C} & 0.000011 & 5.487434 & -2.076405 \\ \mathrm{C} & 0.000072 & 3.919152 & -2.072488 \\ \mathrm{H} & 0.88958 & 5.948207 & -2.523978 \\ \mathrm{H} & -0.889586 & 5.948145 & -2.523989 \\ \mathrm{H} & 0.890073 & 3.457055 & -2.51686 \\ \mathrm{H} & -0.889869 & 3.456992 & -2.516913 \\ \mathrm{C} & 0.000016 & 4.021138 & -0.553471 \\ \mathrm{C} & 0.000004 & 3.026783 & 0.474104 \\ \mathrm{H} & -0.000025 & 3.376036 & 1.506724 \\ \mathrm{C} & 0.000027 & 1.683643 & 0.239039 \\ \mathrm{H} & 0.000052 & 1.335758 & -0.79414 \\ \mathrm{C} & -0.000074 & 0.784018 & 2.780853 \\ \mathrm{C} & 0.000011 & 0.688659 & 1.262325 \\ \mathrm{C} & -0.000011 & -0.688659 & 1.262325\end{array}$

C 0.000074

H $\quad 0.889539$

$-0.784018$

2.780853

H $\quad-0.889803$

1.245139

3.22748

$\mathrm{H} \quad 0.889803$

1.244986

3.227411

$-1.244986$

3.227411

H $\quad-0.889539$

$-1.245139$

3.22748

C $\quad-0.000027$

$-1.683643$

0.239039

$\mathrm{H} \quad-0.000052$

$-1.335758$

$-0.79414$

C $\quad-0.000004$

$-3.026783$

0.474104

H 0.000025

$-3.376036$

1.506724

C $\quad-0.000016$

$-4.021138$

$-0.553471$

C $\quad-0.000072$

$-3.919152$

$-2.072488$

C $\quad-0.000011$

$-5.487434$

$-2.076405$

H 0.889869

$-3.456992$

$-2.516913$

H $\quad-0.890073$

$-3.457055$

$-2.51686$

H 0.889586

$-5.948145$

$-2.523989$

$-5.948207$

$-2.523978$

C $\quad-0.000012$

$-5.39294$

$-0.55984$

C $\quad 0.000012$

$-6.401755$

0.468367

$\mathrm{H} \quad 0.00002$

$-6.048626$

1.499865

C $\quad 0.000027$

$-7.725832$

0.230463

H $\quad 0.000047$

$-8.450419$

1.038855

$\mathrm{H} \quad 0.000021$

$-8.12148$

$-0.782137$

H $\quad-0.000047$

8.450419

1.038855

$\mathbf{A}(\mathrm{DP}=5)$

C $\quad-12.439764$

0.08603

0.000025

H $\quad-12.833443$

$-0.927336$

$-0.000011$

C $\quad-11.116013$

0.326534

0.000032

H $\quad-10.765021$

1.358768

0.000068

C $\quad-10.105241$

$-0.699374$

$-0.000008$

C $\quad-10.196436$

$-2.216072$

$-0.000086$

C $\quad-8.628182$

$-2.208986$

$-0.000037$

H $\quad-10.656247$

$-2.664607$

$-0.88969$

H $\quad-10.656292$

$-2.664686$

0.889452

$\mathrm{H} \quad-8.165178$

$-2.652438$

$-0.890009$

$\mathrm{H} \quad-8.165223$

$-2.652466$

0.889947

C $\quad-8.732976$

$-0.690081$

$-0.000026$

C $\quad-5.501376$

2.648253

0.000024

C $\quad-5.404323$

1.129958

0.000009

C $\quad-4.0249$

1.131958

0.000006 


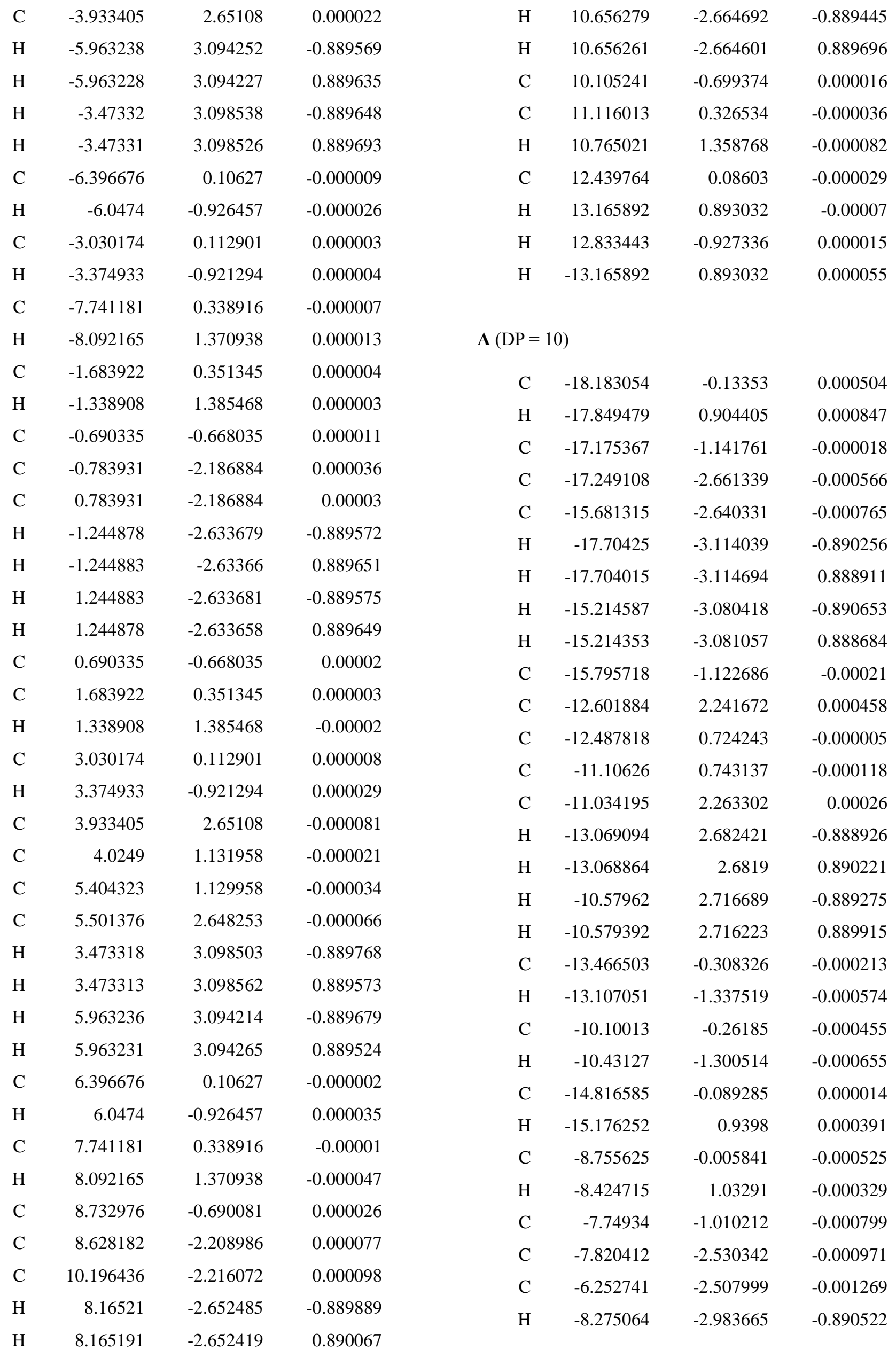




\begin{tabular}{|c|c|c|c|c|c|c|c|}
\hline $\mathrm{H}$ & -8.274758 & -2.983927 & 0.888606 & $\mathrm{C}$ & 10.10013 & 0.261853 & -0.000328 \\
\hline $\mathrm{H}$ & -5.785651 & -2.948039 & -0.891036 & $\mathrm{H}$ & 10.431271 & 1.300516 & -0.00056 \\
\hline $\mathrm{H}$ & -5.785343 & -2.948473 & 0.888117 & $\mathrm{C}$ & 11.106259 & -0.743135 & 0.000016 \\
\hline $\mathrm{C}$ & -6.367145 & -0.990433 & -0.000843 & $\mathrm{C}$ & 11.034193 & -2.2633 & 0.000459 \\
\hline $\mathrm{C}$ & -5.389815 & 0.041862 & -0.0007 & $\mathrm{C}$ & 12.601882 & -2.241671 & 0.000566 \\
\hline $\mathrm{H}$ & -5.749516 & 1.070953 & -0.000514 & $\mathrm{H}$ & 10.579572 & -2.716715 & -0.889038 \\
\hline $\mathrm{C}$ & -4.03831 & -0.176502 & -0.000802 & $\mathrm{H}$ & 10.579436 & -2.716192 & 0.890153 \\
\hline $\mathrm{H}$ & -3.678613 & -1.205597 & -0.000969 & $\mathrm{H}$ & 13.069045 & -2.68242 & -0.888843 \\
\hline $\mathrm{C}$ & -3.175562 & 2.373273 & -0.000486 & $\mathrm{H}$ & 13.068908 & -2.681899 & 0.890305 \\
\hline $\mathrm{C}$ & -3.061075 & 0.855739 & -0.000697 & $\mathrm{C}$ & 12.487818 & -0.724242 & 0.00012 \\
\hline $\mathrm{C}$ & -1.678662 & 0.87561 & -0.000671 & $\mathrm{C}$ & 13.466504 & 0.308327 & -0.000114 \\
\hline $\mathrm{C}$ & -1.607906 & 2.395811 & -0.000648 & $\mathrm{H}$ & 13.107052 & 1.337519 & -0.000477 \\
\hline $\mathrm{H}$ & -3.642954 & 2.813606 & -0.889954 & $\mathrm{C}$ & 14.816585 & 0.089284 & 0.000083 \\
\hline $\mathrm{H}$ & -3.642782 & 2.813399 & 0.889178 & $\mathrm{H}$ & 15.176251 & -0.939801 & 0.00046 \\
\hline $\mathrm{H}$ & -1.153563 & 2.849345 & -0.890241 & $\mathrm{C}$ & 15.681318 & 2.640329 & -0.00072 \\
\hline $\mathrm{H}$ & -1.153394 & 2.849282 & 0.888889 & $\mathrm{C}$ & 15.79572 & 1.122685 & -0.00017 \\
\hline $\mathrm{C}$ & -0.672416 & -0.128278 & -0.000701 & $\mathrm{C}$ & 17.175369 & 1.141758 & 0 \\
\hline $\mathrm{H}$ & -1.002902 & -1.167158 & -0.000704 & $\mathrm{C}$ & 17.24911 & 2.661336 & -0.00059 \\
\hline $\mathrm{C}$ & 0.672416 & 0.128284 & -0.000714 & $\mathrm{H}$ & 15.214553 & 3.080413 & -0.89059 \\
\hline $\mathrm{H}$ & 1.002902 & 1.167165 & -0.000726 & $\mathrm{H}$ & 15.214392 & 3.081059 & 0.888747 \\
\hline $\mathrm{C}$ & 1.678662 & -0.875604 & -0.000685 & $\mathrm{H}$ & 17.704217 & 3.114016 & -0.890309 \\
\hline $\mathrm{C}$ & 1.607906 & -2.395805 & -0.000569 & $\mathrm{H}$ & 17.704055 & 3.114709 & 0.888859 \\
\hline $\mathrm{C}$ & 3.175562 & -2.373268 & -0.000614 & $\mathrm{C}$ & 18.183054 & 0.133525 & 0.000518 \\
\hline $\mathrm{H}$ & 1.15346 & -2.849368 & -0.890092 & $\mathrm{H}$ & 17.849478 & -0.904408 & 0.000886 \\
\hline $\mathrm{H}$ & 1.153496 & -2.849246 & 0.889037 & $\mathrm{C}$ & 19.523983 & 0.386695 & 0.000584 \\
\hline $\mathrm{H}$ & 3.642851 & -2.813538 & -0.890169 & $\mathrm{H}$ & 19.859142 & 1.423991 & 0.000212 \\
\hline $\mathrm{H}$ & 3.642885 & -2.813455 & 0.888963 & $\mathrm{C}$ & 20.531577 & -0.626631 & 0.001121 \\
\hline $\mathrm{C}$ & 3.061075 & -0.855733 & -0.00067 & $\mathrm{C}$ & 20.450871 & -2.147018 & 0.001751 \\
\hline $\mathrm{C}$ & 4.03831 & 0.176507 & -0.000735 & $\mathrm{C}$ & 22.019057 & -2.129301 & 0.001923 \\
\hline $\mathrm{H}$ & 3.678614 & 1.205603 & -0.00085 & $\mathrm{H}$ & 19.995087 & -2.598148 & -0.888094 \\
\hline $\mathrm{C}$ & 6.252742 & 2.508004 & -0.001004 & $\mathrm{H}$ & 19.994879 & -2.597398 & 0.891869 \\
\hline $\mathrm{C}$ & 6.367145 & 0.990438 & -0.000768 & $\mathrm{H}$ & 22.486036 & -2.570953 & -0.887405 \\
\hline $\mathrm{C}$ & 7.749341 & 1.010217 & -0.000658 & $\mathrm{H}$ & 22.485828 & -2.570215 & 0.891726 \\
\hline $\mathrm{C}$ & 7.820413 & 2.530346 & -0.000985 & $\mathrm{C}$ & 21.903912 & -0.614225 & 0.001288 \\
\hline $\mathrm{H}$ & 5.785514 & 2.948116 & -0.89066 & $\mathrm{C}$ & 22.898215 & 0.427601 & 0.00098 \\
\hline $\mathrm{H}$ & 5.785483 & 2.948406 & 0.888493 & $\mathrm{H}$ & 22.530821 & 1.454122 & 0.000514 \\
\hline $\mathrm{H}$ & 8.27493 & 2.983613 & -0.890636 & $\mathrm{C}$ & 24.225654 & 0.208205 & 0.001227 \\
\hline $\mathrm{H}$ & 8.274896 & 2.983986 & 0.888492 & $\mathrm{H}$ & 24.938875 & 1.026644 & 0.000976 \\
\hline $\mathrm{C}$ & 5.389815 & -0.041857 & -0.00067 & $\mathrm{H}$ & 24.635378 & -0.798771 & 0.001689 \\
\hline $\mathrm{H}$ & 5.749516 & -1.070947 & -0.000534 & $\mathrm{C}$ & -22.898215 & -0.427611 & 0.001117 \\
\hline $\mathrm{C}$ & 8.755625 & 0.005844 & -0.000363 & $\mathrm{H}$ & -22.53082 & -1.454131 & 0.00067 \\
\hline $\mathrm{H}$ & 8.424714 & -1.032907 & -0.000141 & $\mathrm{C}$ & -22.019059 & 2.129292 & 0.001949 \\
\hline
\end{tabular}




$\begin{array}{rrrr}\mathrm{C} & -21.903913 & 0.614216 & 0.001369 \\ \mathrm{C} & -20.531577 & 0.626624 & 0.001155 \\ \mathrm{C} & -20.450874 & 2.147011 & 0.001771 \\ \mathrm{H} & -22.486044 & 2.570915 & -0.887391 \\ \mathrm{H} & -22.485825 & 2.570236 & 0.89174 \\ \mathrm{H} & -19.995095 & 2.598131 & -0.888082 \\ \mathrm{H} & -19.994876 & 2.597404 & 0.891881 \\ \mathrm{C} & -19.523982 & -0.386701 & 0.000608 \\ \mathrm{C} & -24.225654 & -0.208216 & 0.001392 \\ \mathrm{H} & -24.938874 & -1.026655 & 0.001182 \\ \mathrm{H} & -24.635379 & 0.798759 & 0.001836 \\ \mathrm{H} & -19.85914 & -1.423997 & 0.000264\end{array}$

\begin{tabular}{|c|c|c|c|}
\hline$C$ & 3.878992 & -1.536316 & -0.789069 \\
\hline$C$ & 5.445861 & -1.573267 & -0.764627 \\
\hline $\mathrm{H}$ & 3.424803 & -1.85846 & -1.734311 \\
\hline $\mathrm{H}$ & 3.392585 & -2.079251 & 0.030812 \\
\hline $\mathrm{H}$ & 5.913735 & -1.917347 & -1.695344 \\
\hline $\mathrm{H}$ & 5.880078 & -2.137832 & 0.069818 \\
\hline $\mathrm{C}$ & 5.386176 & -0.06407 & -0.577352 \\
\hline $\mathrm{C}$ & 6.403665 & 0.917496 & -0.431632 \\
\hline $\mathrm{H}$ & 6.088417 & 1.954188 & -0.312157 \\
\hline $\mathrm{C}$ & 7.743675 & 0.635998 & -0.429619 \\
\hline $\mathrm{H}$ & 8.053996 & -0.402409 & -0.547704 \\
\hline $\mathrm{C}$ & 8.739739 & 3.116408 & -0.094329 \\
\hline $\mathrm{C}$ & 8.770957 & 1.606172 & -0.279247 \\
\hline $\mathrm{C}$ & 10.151766 & 1.545033 & -0.239699 \\
\hline $\mathrm{C}$ & 10.305702 & 3.046967 & -0.048967 \\
\hline $\mathrm{H}$ & 8.328234 & 3.68886 & -0.934832 \\
\hline $\mathrm{H}$ & 8.267173 & 3.471265 & 0.829931 \\
\hline $\mathrm{H}$ & 10.814574 & 3.578768 & -0.862439 \\
\hline $\mathrm{H}$ & 10.753345 & 3.36069 & 0.902205 \\
\hline $\mathrm{C}$ & 11.09753 & 0.488681 & -0.335514 \\
\hline $\mathrm{H}$ & 10.70594 & -0.519021 & -0.47605 \\
\hline $\mathrm{C}$ & 12.454662 & 0.654231 & -0.261397 \\
\hline $\mathrm{H}$ & 12.849717 & 1.660384 & -0.119893 \\
\hline $\mathrm{C}$ & 13.392586 & -0.40968 & -0.354668 \\
\hline $\mathrm{C}$ & 13.218471 & -1.909155 & -0.548094 \\
\hline $\mathrm{C}$ & 14.782308 & -2.001593 & -0.489552 \\
\hline $\mathrm{H}$ & 12.774049 & -2.215546 & -1.503164 \\
\hline $\mathrm{H}$ & 12.695172 & -2.433956 & 0.260772 \\
\hline $\mathrm{H}$ & 15.257755 & -2.362683 & -1.409924 \\
\hline $\mathrm{H}$ & 15.178085 & -2.580513 & 0.354097 \\
\hline $\mathrm{C}$ & 14.771885 & -0.491161 & -0.303425 \\
\hline $\mathrm{C}$ & 15.818067 & 0.45676 & -0.138172 \\
\hline $\mathrm{H}$ & 15.532769 & 1.502731 & -0.024078 \\
\hline $\mathrm{C}$ & 18.203845 & 2.588173 & 0.23953 \\
\hline $\mathrm{C}$ & 18.199697 & 1.077615 & 0.054392 \\
\hline $\mathrm{C}$ & 19.577418 & 0.981629 & 0.114246 \\
\hline $\mathrm{C}$ & 19.766578 & 2.479121 & 0.307924 \\
\hline $\mathrm{H}$ & 17.819338 & 3.170733 & -0.606782 \\
\hline $\mathrm{H}$ & 17.726756 & 2.954797 & 1.156841 \\
\hline $\mathrm{H}$ & 20.300646 & 2.997829 & -0.497818 \\
\hline $\mathrm{H}$ & 20.208042 & 2.781432 & 1.265672 \\
\hline $\mathrm{C}$ & 17.148632 & 0.135546 & -0.11309 \\
\hline
\end{tabular}




\begin{tabular}{|c|c|c|c|c|c|c|c|}
\hline $\mathrm{H}$ & 17.431377 & -0.911217 & -0.226563 & $\mathrm{C}$ & -11.097525 & 0.488853 & -0.335212 \\
\hline $\mathrm{C}$ & 20.49958 & -0.09714 & 0.030549 & $\mathrm{H}$ & -10.705931 & -0.518848 & -0.475741 \\
\hline $\mathrm{H}$ & 20.087099 & -1.09564 & -0.115828 & $\mathrm{C}$ & -10.305691 & 3.047134 & -0.048601 \\
\hline $\mathrm{C}$ & 21.858361 & 0.039123 & 0.12205 & $\mathrm{C}$ & -10.151762 & 1.545206 & -0.239382 \\
\hline $\mathrm{H}$ & 22.272494 & 1.036833 & 0.26888 & $\mathrm{C}$ & -8.770952 & 1.60634 & -0.278937 \\
\hline $\mathrm{C}$ & 22.776953 & -1.043344 & 0.039591 & $\mathrm{C}$ & -8.739729 & 3.116574 & -0.094007 \\
\hline $\mathrm{C}$ & 22.578443 & -2.539591 & -0.155282 & $\mathrm{H}$ & -10.814595 & 3.578971 & -0.862029 \\
\hline $\mathrm{C}$ & 24.139924 & -2.65903 & -0.080516 & $\mathrm{H}$ & -10.753296 & 3.360816 & 0.902603 \\
\hline $\mathrm{H}$ & 22.13881 & -2.838644 & -1.114872 & $\mathrm{H}$ & -8.328258 & 3.689023 & -0.934528 \\
\hline $\mathrm{H}$ & 22.037758 & -3.054814 & 0.648261 & $\mathrm{H}$ & -8.267128 & 3.471437 & 0.830233 \\
\hline $\mathrm{H}$ & 24.618535 & -3.028679 & -0.995855 & $\mathrm{C}$ & -7.74368 & 0.636161 & -0.429343 \\
\hline $\mathrm{H}$ & 24.517049 & -3.24425 & 0.767329 & $\mathrm{H}$ & -8.054014 & -0.402239 & -0.547454 \\
\hline $\mathrm{C}$ & 24.15307 & -1.148708 & 0.105029 & $\mathrm{C}$ & -33.522538 & -1.911886 & 0.584058 \\
\hline $\mathrm{C}$ & 25.21315 & -0.216166 & 0.279728 & $\mathrm{C}$ & -33.505466 & -3.420199 & 0.401154 \\
\hline $\mathrm{H}$ & 24.941445 & 0.833757 & 0.390941 & $\mathrm{C}$ & -31.944759 & -3.290342 & 0.317098 \\
\hline $\mathrm{C}$ & 26.537681 & -0.554997 & 0.316244 & $\mathrm{H}$ & -33.987776 & -3.794069 & -0.51078 \\
\hline $\mathrm{H}$ & 26.808824 & -1.60509 & 0.205476 & $\mathrm{H}$ & -33.874688 & -4.006453 & 1.252035 \\
\hline $\mathrm{C}$ & 27.616846 & 1.887207 & 0.67609 & $\mathrm{H}$ & -31.508807 & -3.586616 & -0.644828 \\
\hline $\mathrm{C}$ & 27.599646 & 0.376505 & 0.492129 & $\mathrm{H}$ & -31.395986 & -3.799523 & 1.118758 \\
\hline $\mathrm{C}$ & 28.973253 & 0.266925 & 0.562586 & $\mathrm{C}$ & -32.157062 & -1.795242 & 0.511244 \\
\hline $\mathrm{C}$ & 29.1778 & 1.76196 & 0.756405 & $\mathrm{C}$ & -29.177855 & 1.761901 & 0.756143 \\
\hline $\mathrm{H}$ & 27.244765 & 2.472712 & -0.173696 & $\mathrm{C}$ & -28.973276 & 0.26686 & 0.562404 \\
\hline $\mathrm{H}$ & 27.136655 & 2.25929 & 1.589537 & $\mathrm{C}$ & -27.59967 & 0.376461 & 0.491978 \\
\hline $\mathrm{H}$ & 29.723393 & 2.274246 & -0.045795 & $\mathrm{C}$ & -27.6169 & 1.88717 & 0.675881 \\
\hline $\mathrm{H}$ & 29.615333 & 2.060538 & 1.71719 & $\mathrm{H}$ & -29.72342 & 2.274131 & -0.046112 \\
\hline $\mathrm{C}$ & 29.888027 & -0.823617 & 0.486419 & $\mathrm{H}$ & -29.615439 & 2.060526 & 1.716891 \\
\hline $\mathrm{H}$ & 29.466417 & -1.818044 & 0.338102 & $\mathrm{H}$ & -27.24479 & 2.472655 & -0.173906 \\
\hline $\mathrm{C}$ & 31.243199 & -0.699158 & 0.587006 & $\mathrm{H}$ & -27.136754 & 2.259286 & 1.589338 \\
\hline $\mathrm{H}$ & 31.666457 & 0.2944 & 0.735532 & $\mathrm{C}$ & -29.888029 & -0.823703 & 0.486281 \\
\hline $\mathrm{C}$ & 32.157075 & -1.795121 & 0.511365 & $\mathrm{H}$ & -29.466399 & -1.818131 & 0.338027 \\
\hline $\mathrm{C}$ & 31.944795 & -3.290212 & 0.317133 & $\mathrm{C}$ & -26.537683 & -0.555025 & 0.316143 \\
\hline $\mathrm{C}$ & 33.505502 & -3.420054 & 0.401221 & $\mathrm{H}$ & -26.808803 & -1.605124 & 0.205374 \\
\hline $\mathrm{H}$ & 31.50887 & -3.586445 & -0.644817 & $\mathrm{C}$ & -31.243205 & -0.699262 & 0.586837 \\
\hline $\mathrm{H}$ & 31.396011 & -3.799441 & 1.118755 & $\mathrm{H}$ & -31.666482 & 0.294299 & 0.735296 \\
\hline $\mathrm{H}$ & 33.987839 & -3.793882 & -0.510716 & $\mathrm{C}$ & -25.213156 & -0.216171 & 0.279671 \\
\hline $\mathrm{H}$ & 33.87471 & -4.006338 & 1.252088 & $\mathrm{H}$ & -24.941474 & 0.833759 & 0.390881 \\
\hline $\mathrm{C}$ & 33.522552 & -1.911748 & 0.584185 & $\mathrm{C}$ & -24.153053 & -1.148696 & 0.105021 \\
\hline $\mathrm{C}$ & 34.600969 & -0.974738 & 0.765328 & $\mathrm{C}$ & -24.139873 & -2.659024 & -0.080476 \\
\hline $\mathrm{H}$ & 34.323586 & 0.074247 & 0.8723 & $\mathrm{C}$ & -22.578392 & -2.539558 & -0.155201 \\
\hline $\mathrm{C}$ & 35.902078 & -1.314398 & 0.808473 & $\mathrm{H}$ & -24.618447 & -3.028703 & -0.995823 \\
\hline $\mathrm{H}$ & 36.680878 & -0.570869 & 0.946993 & $\mathrm{H}$ & -24.517017 & -3.244233 & 0.767369 \\
\hline $\mathrm{H}$ & 36.224195 & -2.347776 & 0.706483 & $\mathrm{H}$ & -22.138721 & -2.83863 & -1.114767 \\
\hline
\end{tabular}




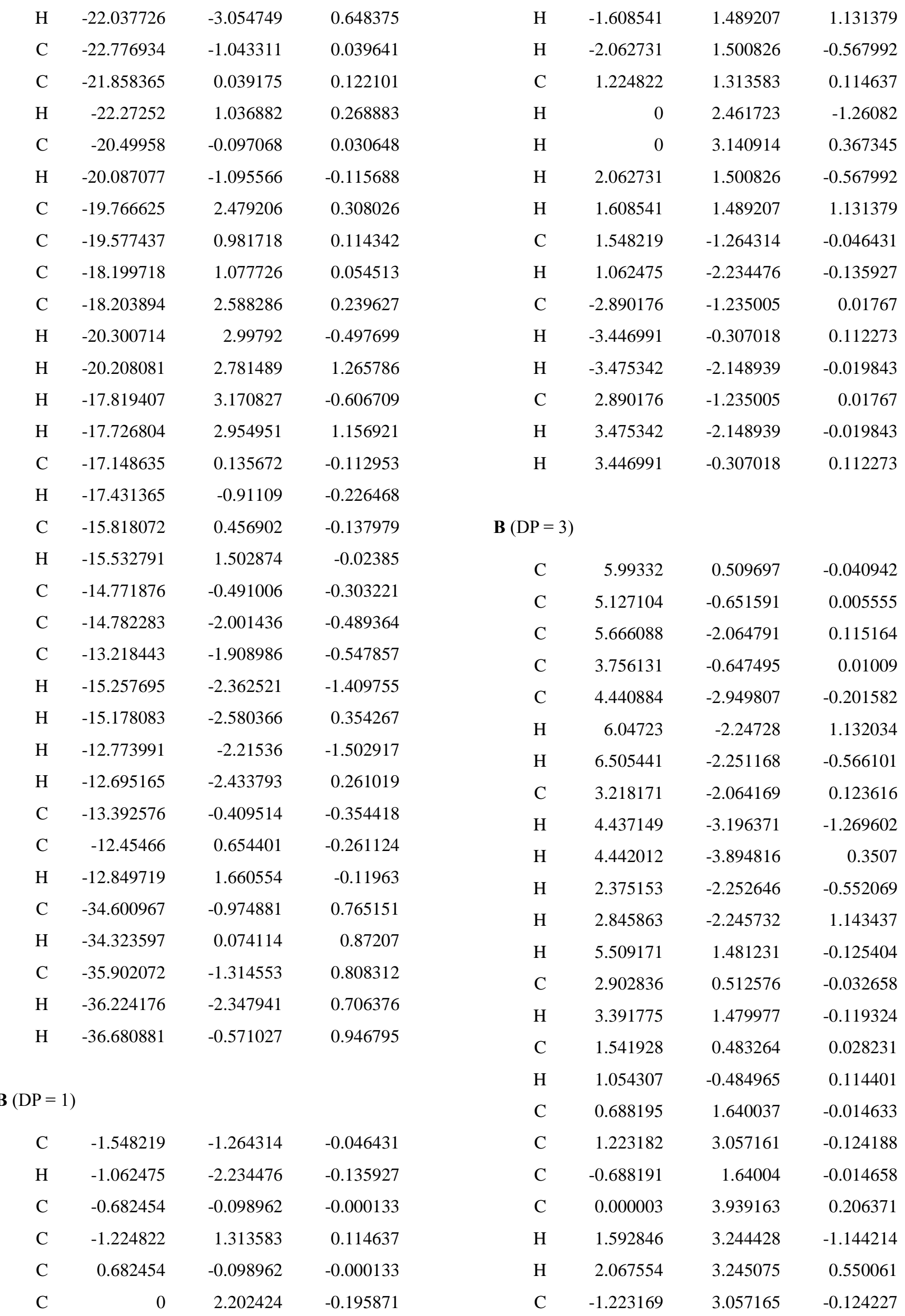




\begin{tabular}{|c|c|c|c|c|c|c|c|}
\hline $\mathrm{H}$ & -0.000014 & 4.173993 & 1.277072 & $\mathrm{H}$ & 9.038377 & -3.210469 & 1.504482 \\
\hline $\mathrm{H}$ & 0.000013 & 4.889995 & -0.335834 & $\mathrm{H}$ & 7.195912 & -2.549813 & -0.824911 \\
\hline $\mathrm{H}$ & -2.067561 & 3.245082 & 0.549996 & $\mathrm{C}$ & 7.350704 & 0.329123 & -0.001126 \\
\hline $\mathrm{H}$ & -1.592803 & 3.244434 & -1.144263 & $\mathrm{H}$ & 7.836713 & 1.298057 & -0.086447 \\
\hline $\mathrm{C}$ & -1.541929 & 0.483267 & 0.02817 & $\mathrm{C}$ & 5.990133 & 0.297052 & 0.076856 \\
\hline $\mathrm{H}$ & -1.054309 & -0.484965 & 0.114304 & $\mathrm{H}$ & 5.504689 & -0.673375 & 0.149756 \\
\hline $\mathrm{C}$ & -2.902837 & 0.512588 & -0.032713 & $\mathrm{C}$ & 5.135143 & 1.452141 & 0.05648 \\
\hline $\mathrm{H}$ & -3.391772 & 1.479991 & -0.119367 & $\mathrm{C}$ & 5.667234 & 2.871307 & -0.038559 \\
\hline $\mathrm{C}$ & -3.756135 & -0.647486 & 0.009997 & $\mathrm{C}$ & 3.757256 & 1.450262 & 0.065974 \\
\hline $\mathrm{C}$ & -5.127105 & -0.651586 & 0.005634 & $\mathrm{C}$ & 4.445709 & 3.746905 & 0.313787 \\
\hline $\mathrm{C}$ & -3.218156 & -2.064174 & 0.123274 & $\mathrm{H}$ & 6.027955 & 3.073119 & -1.059083 \\
\hline $\mathrm{C}$ & -5.666092 & -2.064799 & 0.115019 & $\mathrm{H}$ & 6.517038 & 3.051519 & 0.630983 \\
\hline $\mathrm{C}$ & -4.440901 & -2.949783 & -0.201893 & $\mathrm{C}$ & 3.221522 & 2.868936 & -0.022978 \\
\hline $\mathrm{H}$ & -2.845712 & -2.245872 & 1.143023 & $\mathrm{H}$ & 4.452352 & 3.964637 & 1.388098 \\
\hline $\mathrm{H}$ & -6.047242 & -2.24745 & 1.131854 & $\mathrm{H}$ & 4.441446 & 4.706261 & -0.213226 \\
\hline $\mathrm{H}$ & -6.505447 & -2.251058 & -0.566278 & $\mathrm{H}$ & 2.379811 & 3.048002 & 0.656901 \\
\hline $\mathrm{H}$ & -4.44199 & -3.894825 & 0.35033 & $\mathrm{H}$ & 2.848239 & 3.069412 & -1.039148 \\
\hline $\mathrm{H}$ & -4.437256 & -3.196288 & -1.269926 & $\mathrm{C}$ & 2.906016 & 0.294318 & 0.101365 \\
\hline $\mathrm{C}$ & -5.993318 & 0.509727 & -0.040504 & $\mathrm{H}$ & 3.394017 & -0.674624 & 0.176613 \\
\hline $\mathrm{H}$ & -5.509168 & 1.481282 & -0.124679 & $\mathrm{C}$ & 1.542157 & 0.323605 & 0.045465 \\
\hline $\mathrm{C}$ & -7.336899 & 0.479712 & 0.017038 & $\mathrm{C}$ & 0.689919 & -0.831006 & 0.079905 \\
\hline $\mathrm{H}$ & -7.922043 & 1.393595 & -0.020712 & $\mathrm{C}$ & 1.222831 & -2.249327 & 0.186597 \\
\hline $\mathrm{H}$ & -7.893829 & -0.448724 & 0.105987 & $\mathrm{C}$ & -0.689019 & -0.831125 & 0.070945 \\
\hline $\mathrm{H}$ & -2.375225 & -2.252543 & -0.552546 & $\mathrm{C}$ & 0.00204 & -3.128953 & -0.158086 \\
\hline $\mathrm{C}$ & 7.336892 & 0.479729 & 0.01683 & $\mathrm{H}$ & 1.583833 & -2.442165 & 1.208696 \\
\hline $\mathrm{H}$ & 7.922012 & 1.393622 & -0.021059 & $\mathrm{H}$ & 2.072456 & -2.434894 & -0.481622 \\
\hline \multirow[t]{2}{*}{$\mathrm{H}$} & 7.89383 & -0.448659 & 0.106224 & $\mathrm{C}$ & -1.222938 & -2.249501 & 0.171805 \\
\hline & & & & $\mathrm{H}$ & 0.008523 & -3.355492 & -1.230587 \\
\hline$(\mathrm{DP}=5)$ & & & & $\mathrm{H}$ & -0.001108 & -4.083962 & 0.3768 \\
\hline $\mathrm{C}$ & 11.755616 & 0.27504 & -0.510573 & $\mathrm{H}$ & -2.064577 & -2.435455 & -0.506335 \\
\hline $\mathrm{H}$ & 12.308625 & -0.659294 & -0.546136 & $\mathrm{H}$ & -1.595871 & -2.442117 & 1.189653 \\
\hline $\mathrm{C}$ & 10.425189 & 0.316466 & -0.315775 & $\mathrm{H}$ & 1.054998 & 1.292837 & -0.031457 \\
\hline $\mathrm{C}$ & 9.569499 & -0.838673 & -0.131178 & $\mathrm{C}$ & -1.540989 & 0.32323 & 0.023298 \\
\hline $\mathrm{C}$ & 10.106752 & -2.256729 & -0.12575 & $\mathrm{H}$ & -1.053188 & 1.292267 & -0.052105 \\
\hline $\mathrm{C}$ & 8.205182 & -0.830167 & 0.007076 & $\mathrm{C}$ & -2.905376 & 0.293726 & 0.064238 \\
\hline $\mathrm{C}$ & 8.928858 & -3.093036 & 0.420223 & $\mathrm{H}$ & -3.393722 & -0.675096 & 0.138659 \\
\hline $\mathrm{H}$ & 11.013466 & -2.363043 & 0.482493 & C & -3.756738 & 1.449018 & 0.01352 \\
\hline $\mathrm{H}$ & 10.382654 & -2.561605 & -1.147285 & C & -3.220846 & 2.867175 & -0.082277 \\
\hline $\mathrm{C}$ & 7.67108 & -2.248252 & 0.121041 & $\mathrm{C}$ & -5.134524 & 1.450135 & -0.007184 \\
\hline $\mathrm{H}$ & 8.88116 & -4.097193 & -0.012731 & $\mathrm{C}$ & -4.448181 & 3.747353 & 0.236857 \\
\hline $\mathrm{H}$ & 6.904946 & -2.350619 & 0.899085 & $\mathrm{H}$ & -2.838925 & 3.05954 & -1.09679 \\
\hline & & & & $\mathrm{H}$ & -2.38506 & 3.052183 & 0.60331 \\
\hline
\end{tabular}




$\begin{array}{rrrr}\mathrm{C} & -5.666428 & 2.868216 & -0.118112 \\ \mathrm{H} & -4.463849 & 3.97419 & 1.30919 \\ \mathrm{H} & -4.439922 & 4.7022 & -0.298237 \\ \mathrm{H} & -6.521599 & 3.053514 & 0.543144 \\ \mathrm{H} & -6.01916 & 3.061464 & -1.143069 \\ \mathrm{C} & -5.988889 & 0.294656 & 0.017228 \\ \mathrm{H} & -5.503258 & -0.674315 & 0.10649 \\ \mathrm{C} & -7.34926 & 0.324828 & -0.064871 \\ \mathrm{H} & -7.83625 & 1.29289 & -0.154917 \\ \mathrm{C} & -8.203352 & -0.83459 & -0.040696 \\ \mathrm{C} & -9.574377 & -0.838151 & -0.067687 \\ \mathrm{C} & -7.668037 & -2.252101 & 0.075733 \\ \mathrm{C} & -10.115477 & -2.251539 & 0.027741 \\ \mathrm{C} & -8.88569 & -3.135761 & -0.272934 \\ \mathrm{H} & -7.31237 & -2.438062 & 1.100674 \\ \mathrm{H} & -10.512775 & -2.438005 & 1.037689 \\ \mathrm{H} & -10.94394 & -2.434997 & -0.667528 \\ \mathrm{H} & -8.8961 & -4.083138 & 0.275203 \\ \mathrm{H} & -8.864814 & -3.377792 & -1.341796 \\ \mathrm{C} & -10.439135 & 0.323579 & -0.124137 \\ \mathrm{H} & -9.953184 & 1.295204 & -0.196572 \\ \mathrm{C} & -11.783596 & 0.294218 & -0.089406 \\ \mathrm{H} & -12.367464 & 1.208625 & -0.133673 \\ \mathrm{H} & -12.342526 & -0.634152 & -0.01327 \\ \mathrm{H} & -6.814284 & -2.438259 & -0.586979 \\ \mathrm{H} & 12.332988 & 1.184813 & -0.644497 \\ & 9.943844 & 1.29293 & -0.298337\end{array}$

B $(\mathrm{DP}=10)$

$\begin{array}{lrrr}\text { C } & 1.567215 & -1.072482 & -1.270489 \\ \mathrm{C} & 1.070132 & -2.506154 & -1.338926 \\ \mathrm{C} & 2.946065 & -1.039628 & -1.225545 \\ \mathrm{C} & 2.325604 & -3.313381 & -1.732193 \\ \mathrm{H} & 0.249213 & -2.636281 & -2.054446 \\ \mathrm{H} & 0.679849 & -2.819442 & -0.35817 \\ \mathrm{C} & 3.513944 & -2.447977 & -1.26128 \\ \mathrm{H} & 2.33609 & -4.321637 & -1.306227 \\ \mathrm{H} & 4.383472 & -2.538195 & -1.923547 \\ \mathrm{H} & 2.36276 & -3.419256 & -2.822592 \\ \mathrm{H} & 3.856251 & -2.744163 & -0.257578 \\ \mathrm{C} & 3.766729 & 0.130629 & -1.105321\end{array}$

\begin{tabular}{|c|c|c|c|}
\hline F & 3.25659 & 1.090963 & -1.089749 \\
\hline C & 5.12845 & 0.125845 & -0.993287 \\
\hline $\mathrm{H}$ & 5.63731 & -0.83525 & -0.995084 \\
\hline C & 5.947887 & 1.295093 & -0.855429 \\
\hline C & 5.381218 & 2.704186 & -0.835424 \\
\hline C & 7.3154 & 1.320527 & -0.672455 \\
\hline $\mathrm{C}$ & 6.622577 & 3.604384 & -1.013231 \\
\hline $\mathrm{H}$ & 4.877154 & 2.8964 & 0.124402 \\
\hline $\mathrm{H}$ & 4.630453 & 2.870334 & -1.617477 \\
\hline $\mathrm{C}$ & 7.805066 & 2.749165 & -0.509982 \\
\hline $\mathrm{H}$ & 6.761403 & 3.826186 & -2.077707 \\
\hline $\mathrm{H}$ & 6.534604 & 4.561578 & -0.489632 \\
\hline $\mathrm{H}$ & 8.729007 & 2.946744 & -1.066832 \\
\hline $\mathrm{H}$ & 8.030338 & 2.954728 & 0.547986 \\
\hline $\mathrm{C}$ & 8.184872 & 0.182719 & -0.588852 \\
\hline $\mathrm{H}$ & 7.731546 & -0.795182 & -0.732394 \\
\hline $\mathrm{C}$ & 9.527304 & 0.236963 & -0.341183 \\
\hline C & 10.395927 & -0.901761 & -0.256341 \\
\hline $\mathrm{C}$ & 9.908768 & -2.328188 & -0.444511 \\
\hline $\mathrm{C}$ & 11.759313 & -0.879134 & -0.047004 \\
\hline $\mathrm{C}$ & 11.08277 & -3.190852 & 0.065903 \\
\hline $\mathrm{H}$ & 9.698979 & -2.519617 & -1.508236 \\
\hline $\mathrm{H}$ & 8.976609 & -2.532881 & 0.095813 \\
\hline $\mathrm{C}$ & 12.326851 & -2.287681 & -0.07409 \\
\hline $\mathrm{H}$ & 10.924826 & -3.430437 & 1.123826 \\
\hline $\mathrm{H}$ & 11.180849 & -4.139235 & -0.471717 \\
\hline $\mathrm{H}$ & 13.06066 & -2.46447 & 0.721577 \\
\hline $\mathrm{H}$ & 12.851768 & -2.466721 & -1.025231 \\
\hline $\mathrm{H}$ & 9.980236 & 1.214704 & -0.195032 \\
\hline C & 12.575375 & 0.288203 & 0.129085 \\
\hline $\mathrm{H}$ & 12.066286 & 1.249185 & 0.134353 \\
\hline $\mathrm{C}$ & 13.93139 & 0.279998 & 0.290332 \\
\hline $\mathrm{H}$ & 14.440218 & -0.681042 & 0.285891 \\
\hline C & 14.747735 & 1.447322 & 0.470333 \\
\hline C & 14.18109 & 2.856435 & 0.494306 \\
\hline C & 16.107985 & 1.468712 & 0.690836 \\
\hline C & 15.427442 & 3.758623 & 0.367358 \\
\hline $\mathrm{H}$ & 13.647665 & 3.034147 & 1.44088 \\
\hline $\mathrm{H}$ & 13.454773 & 3.034812 & -0.307835 \\
\hline C & 16.596207 & 2.893379 & 0.88576 \\
\hline $\mathrm{H}$ & 15.594884 & 4.000714 & -0.688517 \\
\hline $\mathrm{H}$ & 15.325657 & 4.705734 & 0.906493 \\
\hline
\end{tabular}




\begin{tabular}{|c|c|c|c|c|c|c|c|}
\hline $\mathrm{H}$ & 17.533202 & 3.097091 & 0.353314 & $\mathrm{C}$ & -10.413201 & 0.893925 & -0.327905 \\
\hline $\mathrm{H}$ & 16.798011 & 3.082791 & 1.951466 & $\mathrm{C}$ & -11.188926 & 3.154284 & -0.644409 \\
\hline $\mathrm{C}$ & 16.975032 & 0.326404 & 0.783305 & $\mathrm{H}$ & -12.604512 & 2.536265 & 0.922593 \\
\hline $\mathrm{H}$ & 16.522499 & -0.64908 & 0.621948 & $\mathrm{H}$ & -13.260545 & 2.395194 & -0.704017 \\
\hline $\mathrm{C}$ & 18.30948 & 0.375814 & 1.05772 & $\mathrm{C}$ & -9.91248 & 2.327036 & -0.381736 \\
\hline $\mathrm{H}$ & 18.763883 & 1.350177 & 1.219962 & $\mathrm{H}$ & -11.31035 & 3.302547 & -1.72369 \\
\hline $\mathrm{C}$ & 19.175189 & -0.771482 & 1.149707 & $\mathrm{H}$ & -11.158853 & 4.145342 & -0.18067 \\
\hline $\mathrm{C}$ & 20.527924 & -0.757181 & 1.374467 & $\mathrm{H}$ & -9.149526 & 2.480941 & -1.154348 \\
\hline $\mathrm{C}$ & 18.682118 & -2.194796 & 0.948306 & $\mathrm{H}$ & -9.444179 & 2.600944 & 0.576306 \\
\hline $\mathrm{C}$ & 21.097702 & -2.16215 & 1.350217 & $\mathrm{C}$ & -9.538006 & -0.241506 & -0.385508 \\
\hline $\mathrm{C}$ & 19.850625 & -3.066746 & 1.457933 & $\mathrm{H}$ & -10.002664 & -1.224165 & -0.353822 \\
\hline $\mathrm{H}$ & 18.475417 & -2.37567 & -0.117598 & $\mathrm{C}$ & -8.17636 & -0.177658 & -0.476393 \\
\hline $\mathrm{H}$ & 21.645363 & -2.33358 & 0.410376 & $\mathrm{C}$ & -7.299006 & -1.310801 & -0.539408 \\
\hline $\mathrm{H}$ & 21.815132 & -2.340828 & 2.160654 & $\mathrm{C}$ & -7.797762 & -2.745369 & -0.524626 \\
\hline $\mathrm{H}$ & 19.954686 & -4.005354 & 0.904612 & $\mathrm{C}$ & -5.925475 & -1.276506 & -0.668414 \\
\hline $\mathrm{H}$ & 19.679333 & -3.325713 & 2.509115 & $\mathrm{C}$ & -6.523308 & -3.57706 & -0.265538 \\
\hline $\mathrm{C}$ & 21.359054 & 0.415186 & 1.562788 & $\mathrm{H}$ & -8.254324 & -2.997459 & -1.49423 \\
\hline $\mathrm{H}$ & 20.854633 & 1.380057 & 1.568563 & $\mathrm{H}$ & -8.569695 & -2.91854 & 0.234948 \\
\hline $\mathrm{C}$ & 22.694454 & 0.403067 & 1.724996 & $\mathrm{C}$ & -5.363342 & -2.684815 & -0.756807 \\
\hline $\mathrm{H}$ & 23.253166 & 1.324423 & 1.85885 & $\mathrm{H}$ & -6.416117 & -3.752435 & 0.81119 \\
\hline $\mathrm{H}$ & 23.27086 & -0.517693 & 1.726657 & $\mathrm{H}$ & -6.545521 & -4.556161 & -0.754446 \\
\hline $\mathrm{H}$ & 17.746841 & -2.398466 & 1.483595 & $\mathrm{H}$ & -4.453913 & -2.817263 & -0.158433 \\
\hline $\mathrm{C}$ & -18.309285 & -0.400044 & 1.05008 & $\mathrm{H}$ & -5.087496 & -2.917708 & -1.796934 \\
\hline $\mathrm{H}$ & -18.764232 & -1.377309 & 1.192251 & $\mathrm{H}$ & -7.712993 & 0.805675 & -0.505236 \\
\hline $\mathrm{C}$ & -16.982384 & -0.347114 & 0.741827 & $\mathrm{C}$ & -5.10761 & -0.10136 & -0.755472 \\
\hline $\mathrm{C}$ & -16.124059 & -1.489197 & 0.586146 & $\mathrm{H}$ & -5.61356 & 0.857769 & -0.673918 \\
\hline $\mathrm{C}$ & -16.614464 & -2.917839 & 0.74257 & $\mathrm{C}$ & -3.752045 & -0.097861 & -0.927042 \\
\hline $\mathrm{C}$ & -14.770407 & -1.465884 & 0.328421 & $\mathrm{H}$ & -3.24474 & -1.056431 & -1.007033 \\
\hline $\mathrm{C}$ & -15.465727 & -3.768809 & 0.159701 & $\mathrm{C}$ & -2.93509 & 1.078679 & -1.003066 \\
\hline $\mathrm{H}$ & -17.567319 & -3.09751 & 0.230004 & $\mathrm{C}$ & -3.500373 & 2.485397 & -0.908177 \\
\hline $\mathrm{H}$ & -16.787037 & -3.145276 & 1.805972 & $\mathrm{C}$ & -1.56 & 1.116299 & -1.112929 \\
\hline $\mathrm{C}$ & -14.211244 & -2.877388 & 0.283066 & $\mathrm{C}$ & -2.334303 & 3.384027 & -1.372139 \\
\hline $\mathrm{H}$ & -15.353581 & -4.735215 & 0.661248 & $\mathrm{H}$ & -3.792057 & 2.70687 & 0.130138 \\
\hline $\mathrm{H}$ & -13.509822 & -3.029035 & -0.546272 & $\mathrm{H}$ & -4.401053 & 2.623041 & -1.518583 \\
\hline $\mathrm{H}$ & -15.664803 & -3.97203 & -0.898868 & $\mathrm{C}$ & -1.063445 & 2.551798 & -1.098676 \\
\hline $\mathrm{H}$ & -13.651159 & -3.092367 & 1.206214 & $\mathrm{H}$ & -2.423415 & 3.568392 & -2.449029 \\
\hline $\mathrm{C}$ & -13.952475 & -0.296472 & 0.171025 & $\mathrm{H}$ & -2.322088 & 4.358872 & -0.874436 \\
\hline $\mathrm{H}$ & -14.453534 & 0.666741 & 0.230646 & $\mathrm{H}$ & -0.276351 & 2.735831 & -1.839857 \\
\hline $\mathrm{C}$ & -12.60221 & -0.304774 & -0.033143 & $\mathrm{H}$ & -0.628018 & 2.792224 & -0.116402 \\
\hline $\mathrm{H}$ & -12.098689 & -1.267466 & -0.081795 & $\mathrm{H}$ & -16.52973 & 0.631423 & 0.600469 \\
\hline $\mathrm{C}$ & -11.783972 & 0.865623 & -0.175205 & $\mathrm{C}$ & 0.685427 & 0.057172 & -1.211691 \\
\hline $\mathrm{C}$ & -12.343079 & 2.276618 & -0.114942 & $\mathrm{H}$ & 1.146671 & 1.041393 & -1.178209 \\
\hline
\end{tabular}




\begin{tabular}{|c|c|c|c|}
\hline $\mathrm{C}$ & -0.678793 & -0.013364 & -1.181105 \\
\hline $\mathrm{H}$ & -1.139495 & -0.998296 & -1.198796 \\
\hline $\mathrm{C}$ & -19.166474 & 0.747526 & 1.200778 \\
\hline $\mathrm{C}$ & -20.513213 & 0.732314 & 1.458872 \\
\hline $\mathrm{C}$ & -18.671277 & 2.174186 & 1.030537 \\
\hline $\mathrm{H}$ & -17.722158 & 2.357359 & 1.548609 \\
\hline $\mathrm{H}$ & -18.489679 & 2.386321 & -0.034181 \\
\hline $\mathrm{C}$ & -21.07634 & 2.139799 & 1.490729 \\
\hline $\mathrm{H}$ & -21.772945 & 2.297144 & 2.323438 \\
\hline $\mathrm{H}$ & -21.645813 & 2.341548 & 0.570062 \\
\hline $\mathrm{C}$ & -19.822537 & 3.035471 & 1.594614 \\
\hline $\mathrm{H}$ & -19.935463 & 3.990577 & 1.072106 \\
\hline $\mathrm{H}$ & -19.624236 & 3.262399 & 2.648448 \\
\hline $\mathrm{C}$ & -21.345687 & -0.441354 & 1.6325 \\
\hline $\mathrm{H}$ & -20.846279 & -1.408223 & 1.598724 \\
\hline $\mathrm{C}$ & -22.676919 & -0.427709 & 1.82595 \\
\hline $\mathrm{H}$ & -23.248088 & 0.495396 & 1.867229 \\
\hline $\mathrm{H}$ & -23.237387 & -1.349882 & 1.946123 \\
\hline \multicolumn{4}{|c|}{$\mathbf{B}(\mathrm{DP}=15)$} \\
\hline $\mathrm{C}$ & 9.588421 & 1.310985 & -1.157265 \\
\hline $\mathrm{C}$ & 10.112313 & 2.725835 & -0.981025 \\
\hline $\mathrm{C}$ & 8.210507 & 1.31123 & -1.234932 \\
\hline $\mathrm{C}$ & 8.909134 & 3.614692 & -1.361558 \\
\hline $\mathrm{H}$ & 10.994688 & 2.931724 & -1.598896 \\
\hline $\mathrm{H}$ & 10.419759 & 2.888448 & 0.063632 \\
\hline $\mathrm{C}$ & 7.670848 & 2.726277 & -1.117075 \\
\hline $\mathrm{H}$ & 8.878105 & 4.554666 & -0.801482 \\
\hline $\mathrm{H}$ & 6.862218 & 2.933077 & -1.828456 \\
\hline $\mathrm{H}$ & 8.968455 & 3.87036 & -2.425864 \\
\hline $\mathrm{H}$ & 7.250201 & 2.888502 & -0.112576 \\
\hline $\mathrm{C}$ & 7.36362 & 0.16026 & -1.358043 \\
\hline $\mathrm{H}$ & 7.854511 & -0.806967 & -1.436989 \\
\hline $\mathrm{C}$ & 5.997542 & 0.189357 & -1.37247 \\
\hline $\mathrm{H}$ & 5.506759 & 1.155518 & -1.281768 \\
\hline $\mathrm{C}$ & 5.149802 & -0.961973 & -1.485099 \\
\hline $\mathrm{C}$ & 5.688843 & -2.376445 & -1.610424 \\
\hline $\mathrm{C}$ & 3.770171 & -0.970617 & -1.44874 \\
\hline $\mathrm{C}$ & 4.458796 & -3.204059 & -2.040421 \\
\hline $\mathrm{H}$ & 6.087237 & -2.717583 & -0.642369 \\
\hline $\mathrm{H}$ & 6.514155 & -2.453339 & -2.328 \\
\hline
\end{tabular}

\begin{tabular}{|c|c|c|c|}
\hline & 3.24343 & -2.392125 & -1.543822 \\
\hline & 4.429326 & -3.269723 & -3.134213 \\
\hline & 4.475889 & -4.227583 & -1.652821 \\
\hline & 2.380984 & -2.480652 & -2.215468 \\
\hline & 2.90352 & -2.737588 & -0.55519 \\
\hline & 2.915058 & 0.170738 & -1.29692 \\
\hline & 3.397021 & 1.144368 & -1.248427 \\
\hline & 1.552172 & 0.124974 & -1.209495 \\
\hline & 0.697481 & 1.267316 & -1.062187 \\
\hline & 1.224284 & 2.690256 & -0.991042 \\
\hline & -0.681792 & 1.258545 & -1.01436 \\
\hline & 0.011585 & 3.509577 & -0.500174 \\
\hline & 1.559059 & 3.020692 & -1.98651 \\
\hline & 2.090249 & 2.789154 & -0.325362 \\
\hline & -1.220571 & 2.674841 & -0.909639 \\
\hline & 0.047355 & 3.592622 & 0.592248 \\
\hline & -0.00809 & 4.526817 & -0.903864 \\
\hline & -2.041998 & 2.763492 & -0.188424 \\
\hline & -1.624107 & 2.999891 & -1.881095 \\
\hline & 1.070077 & -0.848709 & -1.256705 \\
\hline & -1.529769 & 0.104594 & -1.093721 \\
\hline & -1.039261 & -0.863986 & -1.155688 \\
\hline & -2.895909 & 0.133291 & -1.095277 \\
\hline & -3.385908 & 1.102199 & -1.034268 \\
\hline & -3.744802 & -1.020557 & -1.165811 \\
\hline & -3.20762 & -2.43927 & -1.241178 \\
\hline & -5.124328 & -1.026358 & -1.124808 \\
\hline & -4.440257 & -3.280968 & -1.634826 \\
\hline & -2.805362 & -2.74464 & -0.262866 \\
\hline & -2.385354 & -2.54363 & -1.959425 \\
\hline & -5.652542 & -2.449998 & -1.162838 \\
\hline & -4.474958 & -3.386825 & -2.725312 \\
\hline & -4.422222 & -4.289553 & -1.209898 \\
\hline & -6.518891 & -2.563409 & -1.825641 \\
\hline & -5.986747 & -2.757323 & -0.159771 \\
\hline & -5.97775 & 0.121199 & -1.016612 \\
\hline & -5.494668 & 1.095499 & -1.013863 \\
\hline & -7.34015 & 0.080681 & -0.919787 \\
\hline & -7.823269 & -0.893648 & -0.920967 \\
\hline & -8.19345 & 1.229232 & -0.820552 \\
\hline & -9.572435 & 1.223477 & -0.764968 \\
\hline & -7.666035 & 2.65373 & \\
\hline
\end{tabular}




\begin{tabular}{|c|c|c|c|c|c|c|c|}
\hline $\mathrm{C}$ & -10.110168 & 2.643544 & -0.727532 & $\mathrm{H}$ & 21.090817 & 1.101564 & 0.326624 \\
\hline $\mathrm{C}$ & -8.87601 & 3.496075 & -0.363382 & $\mathrm{C}$ & 19.254264 & 0.103148 & 0.055653 \\
\hline $\mathrm{H}$ & -7.336974 & 2.935516 & -1.832972 & $\mathrm{C}$ & 18.398201 & 1.253972 & 0.072024 \\
\hline $\mathrm{H}$ & -10.515998 & 2.92125 & -1.712634 & $\mathrm{C}$ & 18.918284 & 2.668989 & 0.257681 \\
\hline $\mathrm{H}$ & -10.929506 & 2.767921 & -0.009221 & $\mathrm{C}$ & 17.031501 & 1.261735 & -0.11907 \\
\hline $\mathrm{H}$ & -8.897153 & 4.493166 & -0.814501 & $\mathrm{C}$ & 17.647264 & 3.492793 & 0.554785 \\
\hline $\mathrm{H}$ & -8.835228 & 3.630549 & 0.723741 & $\mathrm{H}$ & 19.4162 & 3.013143 & -0.661995 \\
\hline $\mathrm{C}$ & -10.420836 & 0.067211 & -0.782504 & $\mathrm{H}$ & 19.662336 & 2.745773 & 1.059731 \\
\hline $\mathrm{H}$ & -9.930442 & -0.903347 & -0.795595 & $\mathrm{C}$ & 16.496521 & 2.682924 & -0.07966 \\
\hline $\mathrm{C}$ & -11.78694 & 0.096021 & -0.781229 & $\mathrm{H}$ & 17.496565 & 3.550129 & 1.639049 \\
\hline $\mathrm{H}$ & -12.276818 & 1.066857 & -0.769267 & $\mathrm{H}$ & 17.705357 & 4.519137 & 0.178748 \\
\hline $\mathrm{H}$ & -6.79624 & 2.784336 & -0.165646 & $\mathrm{H}$ & 15.562747 & 2.769422 & 0.488972 \\
\hline $\mathrm{C}$ & 29.348005 & 0.058321 & 1.837666 & $\mathrm{H}$ & 16.272625 & 3.031753 & -1.099663 \\
\hline $\mathrm{H}$ & 29.815531 & 1.020041 & 2.034167 & $\mathrm{H}$ & 18.776439 & -0.864438 & -0.078135 \\
\hline $\mathrm{C}$ & 28.027173 & 0.04273 & 1.500246 & $\mathrm{C}$ & 16.200108 & 0.120943 & -0.373793 \\
\hline $\mathrm{C}$ & 27.191535 & 1.205986 & 1.382722 & $\mathrm{H}$ & 16.685534 & -0.852245 & -0.375999 \\
\hline $\mathrm{C}$ & 27.700234 & 2.615639 & 1.628211 & $\mathrm{C}$ & 14.855762 & 0.1676 & -0.61375 \\
\hline $\mathrm{C}$ & 25.844934 & 1.219977 & 1.089578 & $\mathrm{H}$ & 14.370693 & 1.140956 & -0.611515 \\
\hline $\mathrm{C}$ & 26.580703 & 3.515849 & 1.063068 & $\mathrm{C}$ & 14.023929 & -0.972346 & -0.870242 \\
\hline $\mathrm{H}$ & 28.668841 & 2.806357 & 1.150318 & $\mathrm{C}$ & 14.557742 & -2.393847 & -0.91219 \\
\hline $\mathrm{H}$ & 27.848412 & 2.783091 & 2.706334 & $\mathrm{C}$ & 12.657356 & -0.96291 & -1.063174 \\
\hline $\mathrm{C}$ & 25.309408 & 2.641341 & 1.109646 & $\mathrm{C}$ & 13.407281 & -3.201077 & -1.55056 \\
\hline $\mathrm{H}$ & 26.472021 & 4.45702 & 1.611196 & $\mathrm{H}$ & 14.779711 & -2.745425 & 0.107296 \\
\hline $\mathrm{H}$ & 24.629745 & 2.850238 & 0.274715 & $\mathrm{H}$ & 15.492344 & -2.47982 & -1.479551 \\
\hline $\mathrm{H}$ & 26.809354 & 3.769436 & 0.021454 & $\mathrm{C}$ & 12.136438 & -2.377037 & -1.253222 \\
\hline $\mathrm{H}$ & 24.7323 & 2.815662 & 2.030876 & $\mathrm{H}$ & 13.559635 & -3.255618 & -2.63474 \\
\hline $\mathrm{C}$ & 25.013788 & 0.076002 & 0.840804 & $\mathrm{H}$ & 13.347728 & -4.228384 & -1.177383 \\
\hline $\mathrm{H}$ & 25.499028 & -0.897146 & 0.849525 & $\mathrm{H}$ & 11.3935 & -2.451223 & -2.056542 \\
\hline $\mathrm{C}$ & 23.669341 & 0.120137 & 0.605357 & $\mathrm{H}$ & 11.636951 & -2.723071 & -0.335118 \\
\hline $\mathrm{H}$ & 23.182275 & 1.092467 & 0.608433 & $\mathrm{H}$ & 27.561432 & -0.919992 & 1.303606 \\
\hline $\mathrm{C}$ & 22.836959 & -1.02318 & 0.361821 & $\mathrm{C}$ & 10.443326 & 0.15975 & -1.183728 \\
\hline $\mathrm{C}$ & 23.373044 & -2.443902 & 0.326325 & $\mathrm{H}$ & 9.963942 & -0.807355 & -1.315638 \\
\hline $\mathrm{C}$ & 21.469744 & -1.016858 & 0.177308 & $\mathrm{C}$ & 11.802448 & 0.188449 & -1.04499 \\
\hline $\mathrm{C}$ & 22.219964 & -3.257432 & -0.299283 & $\mathrm{H}$ & 12.280211 & 1.154494 & -0.900192 \\
\hline $\mathrm{H}$ & 23.602047 & -2.788096 & 1.346798 & $\mathrm{C}$ & 30.183262 & -1.109142 & 1.953831 \\
\hline $\mathrm{H}$ & 24.304205 & -2.532136 & -0.246349 & $\mathrm{C}$ & 31.524965 & -1.127677 & 2.237072 \\
\hline $\mathrm{C}$ & 20.949643 & -2.432825 & -0.001128 & $\mathrm{C}$ & 29.668468 & -2.518841 & 1.714616 \\
\hline $\mathrm{H}$ & 22.366288 & -3.320192 & -1.383837 & $\mathrm{H}$ & 28.705559 & -2.706761 & 2.204778 \\
\hline $\mathrm{H}$ & 22.163916 & -4.281899 & 0.082136 & $\mathrm{H}$ & 29.5067 & -2.684491 & 0.63849 \\
\hline $\mathrm{H}$ & 20.203088 & -2.513036 & -0.800502 & $\mathrm{C}$ & 32.063986 & -2.544874 & 2.22093 \\
\hline $\mathrm{H}$ & 20.454661 & -2.773303 & 0.92145 & $\mathrm{H}$ & 32.74305 & -2.748031 & 3.058202 \\
\hline $\mathrm{C}$ & 20.613162 & 0.133828 & 0.193481 & $\mathrm{H}$ & 32.645942 & -2.71922 & 1.302492 \\
\hline
\end{tabular}




\begin{tabular}{|c|c|c|c|c|c|c|c|}
\hline $\mathrm{C}$ & 30.79348 & -3.421572 & 2.266631 & $\mathrm{H}$ & -24.356247 & 2.822965 & 1.391324 \\
\hline $\mathrm{H}$ & 30.900238 & -4.358023 & 1.710162 & $\mathrm{C}$ & -27.71556 & 2.660994 & 1.361813 \\
\hline $\mathrm{H}$ & 30.570718 & -3.684515 & 3.307144 & $\mathrm{H}$ & -26.236168 & 3.753394 & 2.512939 \\
\hline $\mathrm{C}$ & 32.372895 & 0.023506 & 2.474495 & $\mathrm{H}$ & -26.543167 & 4.490456 & 0.939464 \\
\hline $\mathrm{H}$ & 31.890144 & 0.999384 & 2.470184 & $\mathrm{H}$ & -28.409309 & 2.842001 & 2.191763 \\
\hline $\mathrm{C}$ & 33.699577 & -0.020358 & 2.693001 & $\mathrm{H}$ & -28.277531 & 2.867148 & 0.437685 \\
\hline $\mathrm{H}$ & 34.25509 & -0.953752 & 2.708078 & $\mathrm{H}$ & -25.462799 & -0.894615 & 1.083872 \\
\hline $\mathrm{H}$ & 34.27216 & 0.886706 & 2.861409 & $\mathrm{C}$ & -28.018806 & 0.087849 & 1.534539 \\
\hline $\mathrm{C}$ & -17.081857 & 1.206334 & -0.526702 & $\mathrm{H}$ & -27.532296 & -0.884391 & 1.508322 \\
\hline $\mathrm{C}$ & -16.554178 & 2.62863 & -0.603114 & $\mathrm{C}$ & -29.365379 & 0.125081 & 1.74391 \\
\hline $\mathrm{C}$ & -18.451503 & 1.206594 & -0.358203 & $\mathrm{H}$ & -29.853366 & 1.096441 & 1.769734 \\
\hline $\mathrm{C}$ & -17.817247 & 3.477363 & -0.861809 & $\mathrm{C}$ & -30.202783 & -1.030711 & 1.936824 \\
\hline $\mathrm{H}$ & -15.796974 & 2.758481 & -1.38575 & $\mathrm{C}$ & -29.663663 & -2.451404 & 1.924604 \\
\hline $\mathrm{H}$ & -16.070063 & 2.904726 & 0.346433 & $\mathrm{C}$ & -31.548907 & -1.028115 & 2.198735 \\
\hline $\mathrm{C}$ & -18.981996 & 2.629072 & -0.308171 & $\mathrm{C}$ & -30.932376 & -3.327936 & 1.837113 \\
\hline $\mathrm{H}$ & -17.762632 & 4.473383 & -0.411114 & $\mathrm{H}$ & -29.096911 & -2.64814 & 2.847458 \\
\hline $\mathrm{H}$ & -19.903977 & 2.758899 & -0.887701 & $\mathrm{H}$ & -28.973234 & -2.634918 & 1.092547 \\
\hline $\mathrm{H}$ & -17.948265 & 3.614326 & -1.941462 & $\mathrm{C}$ & -32.067967 & -2.439791 & 2.390971 \\
\hline $\mathrm{H}$ & -19.225586 & 2.906102 & 0.729204 & $\mathrm{H}$ & -31.137145 & -3.567173 & 0.787243 \\
\hline $\mathrm{C}$ & -19.290386 & 0.054399 & -0.19539 & $\mathrm{H}$ & -30.832546 & -4.276924 & 2.373255 \\
\hline $\mathrm{H}$ & -18.806045 & -0.918279 & -0.239472 & $\mathrm{H}$ & -33.022108 & -2.612287 & 1.877793 \\
\hline $\mathrm{C}$ & -20.638771 & 0.090337 & 0.021667 & $\mathrm{H}$ & -32.252913 & -2.634217 & 3.458967 \\
\hline $\mathrm{H}$ & -21.12082 & 1.06356 & 0.076355 & $\mathrm{C}$ & -32.399045 & 0.13747 & 2.33744 \\
\hline $\mathrm{C}$ & -21.476516 & -1.061002 & 0.197716 & $\mathrm{H}$ & -31.932455 & 1.108377 & 2.179589 \\
\hline $\mathrm{C}$ & -20.944565 & -2.483246 & 0.162565 & $\mathrm{C}$ & -12.63618 & -1.059754 & -0.789684 \\
\hline $\mathrm{C}$ & -22.829773 & -1.056361 & 0.465885 & $\mathrm{C}$ & -12.099786 & -2.480784 & -0.794062 \\
\hline $\mathrm{C}$ & -22.216331 & -3.351748 & 0.055304 & $\mathrm{C}$ & -14.015496 & -1.062964 & -0.743733 \\
\hline $\mathrm{H}$ & -20.383004 & -2.700365 & 1.084349 & $\mathrm{C}$ & -13.333746 & -3.340411 & -1.14205 \\
\hline $\mathrm{H}$ & -20.251335 & -2.656376 & -0.669428 & $\mathrm{H}$ & -11.696494 & -2.736269 & 0.198027 \\
\hline $\mathrm{C}$ & -23.344231 & -2.474756 & 0.639955 & $\mathrm{H}$ & -11.278648 & -2.622162 & -1.507238 \\
\hline $\mathrm{H}$ & -22.427086 & -3.559088 & -1.000225 & $\mathrm{C}$ & -14.544117 & -2.486501 & -0.707666 \\
\hline $\mathrm{H}$ & -22.118723 & -4.316303 & 0.563452 & $\mathrm{H}$ & -13.37189 & -3.499969 & -2.225879 \\
\hline $\mathrm{H}$ & -24.304602 & -2.642138 & 0.137425 & $\mathrm{H}$ & -13.31471 & -4.326817 & -0.66792 \\
\hline $\mathrm{H}$ & -23.511091 & -2.689307 & 1.706917 & $\mathrm{H}$ & -15.413405 & -2.633046 & -1.360036 \\
\hline $\mathrm{C}$ & -23.665134 & 0.100897 & 0.615211 & $\mathrm{H}$ & -14.873707 & -2.742545 & 0.31121 \\
\hline $\mathrm{H}$ & -23.196317 & 1.070072 & 0.462623 & $\mathrm{C}$ & -16.230011 & 0.053618 & -0.5816 \\
\hline $\mathrm{C}$ & -24.993528 & 0.074264 & 0.930894 & $\mathrm{H}$ & -16.712788 & -0.919248 & -0.526029 \\
\hline $\mathrm{C}$ & -25.829232 & 1.232854 & 1.074949 & $\mathrm{C}$ & -14.868367 & 0.088881 & -0.689614 \\
\hline $\mathrm{C}$ & -25.315011 & 2.649902 & 0.887672 & $\mathrm{H}$ & -14.384833 & 1.062032 & -0.732206 \\
\hline $\mathrm{C}$ & -27.181075 & 1.239782 & 1.342868 & $\mathrm{C}$ & -33.707666 & 0.112155 & 2.648376 \\
\hline $\mathrm{C}$ & -26.445037 & 3.5325 & 1.459803 & $\mathrm{H}$ & -34.282301 & 1.029086 & 2.737926 \\
\hline $\mathrm{H}$ & -25.144956 & 2.852895 & -0.180965 & $\mathrm{H}$ & -34.244701 & -0.815361 & 2.825284 \\
\hline
\end{tabular}




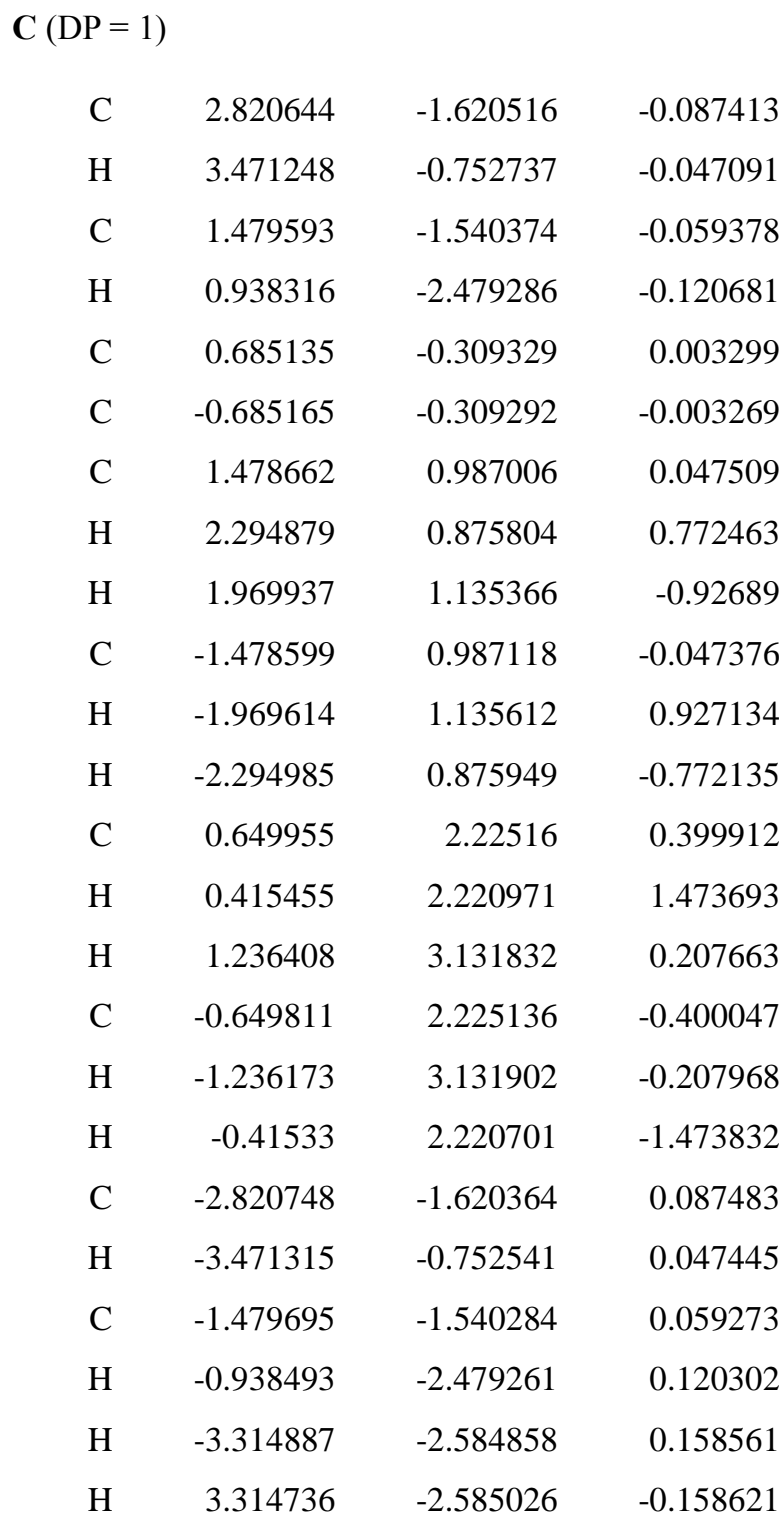

C $(\mathrm{DP}=3)$

$\begin{array}{lrrr}\mathrm{C} & -5.786483 & 0.56602 & 0.037171 \\ \mathrm{H} & -5.256554 & 1.509629 & -0.04878 \\ \mathrm{C} & -4.985123 & -0.658778 & 0.002894 \\ \mathrm{C} & -3.612348 & -0.647963 & -0.089516 \\ \mathrm{C} & -2.830971 & 0.578959 & -0.005986 \\ \mathrm{H} & -3.383906 & 1.498888 & 0.134311 \\ \mathrm{C} & -5.770436 & -1.95957 & 0.046509 \\ \mathrm{H} & -6.531377 & -1.885282 & 0.833943 \\ \mathrm{H} & -6.332797 & -2.067188 & -0.894465 \\ \mathrm{C} & -2.827907 & -1.942872 & -0.255195 \\ \mathrm{H} & -2.270782 & -2.143412 & 0.673327 \\ \mathrm{H} & -2.065199 & -1.797038 & -1.030423 \\ \mathrm{C} & -4.917654 & -3.209022 & 0.280111\end{array}$

\begin{tabular}{|c|c|c|c|}
\hline & -4.604217 & -3.25379 & 1.33262 \\
\hline & -5.51516 & -4.108579 & 0.088613 \\
\hline & -3.681343 & -3.16262 & -0.612455 \\
\hline & -3.082118 & -4.075939 & -0.513717 \\
\hline & -3.994868 & -3.100762 & -1.664051 \\
\hline & -1.47311 & 0.660946 & -0.048159 \\
\hline & -0.689994 & 1.885163 & 0.024347 \\
\hline & 0.689994 & 1.885153 & -0.024433 \\
\hline & -0.921433 & -0.260002 & -0.188505 \\
\hline & 1.473077 & 0.660913 & 0.04796 \\
\hline & 0.921361 & -0.260066 & 0.187945 \\
\hline & 2.830953 & 0.578946 & 0.006053 \\
\hline & 3.38387 & 1.498944 & -0.13387 \\
\hline & 3.612335 & -0.647969 & 0.089468 \\
\hline & 4.985113 & -0.658777 & -0.00294 \\
\hline & 2.827906 & -1.942902 & 0.255051 \\
\hline & 2.06501 & -1.797051 & 1.030083 \\
\hline & 2.271019 & -2.143568 & -0.673591 \\
\hline & 5.770421 & -1.959555 & -0.04654 \\
\hline & 6.332913 & -2.067086 & 0.894369 \\
\hline & 6.531283 & -1.885305 & -0.834066 \\
\hline & 3.681339 & -3.162568 & 0.612585 \\
\hline & 3.994816 & -3.1005 & 1.664184 \\
\hline & 3.082138 & -4.075917 & 0.513988 \\
\hline 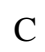 & 4.917676 & -3.209074 & -0.27994 \\
\hline & 5.51524 & -4.108561 & -0.088295 \\
\hline & 4.60425 & -3.254041 & -1.332444 \\
\hline & 7.126699 & 0.640987 & -0.119653 \\
\hline & 7.77009 & -0.229874 & -0.194044 \\
\hline$y$ & 5.786466 & 0.566034 & -0.037285 \\
\hline & 5.256485 & 1.509644 & 0.048313 \\
\hline & 1.474164 & 3.186295 & -0.12166 \\
\hline & 2.019495 & 3.346377 & 0.821656 \\
\hline & 2.247773 & 3.075172 & -0.892258 \\
\hline & -1.474139 & 3.18631 & 0.121661 \\
\hline & -2.247788 & 3.075169 & 0.892214 \\
\hline & -2.019426 & 3.346473 & -0.821669 \\
\hline & 0.625324 & 4.420609 & -0.436367 \\
\hline & 0.329472 & 4.409382 & -1.49483 \\
\hline & 1.220624 & 5.328885 & -0.283146 \\
\hline & -0.625277 & 4.420568 & 0.436516 \\
\hline & -1.220556 & 5.328878 & 0.283414 \\
\hline
\end{tabular}




\begin{tabular}{|c|c|c|c|c|c|c|c|}
\hline $\mathrm{H}$ & -0.329425 & 4.40919 & 1.494977 & $\mathrm{C}$ & -0.624002 & -3.417659 & 0.438707 \\
\hline $\mathrm{C}$ & -7.126701 & 0.641008 & 0.119765 & $\mathrm{H}$ & -0.324947 & -3.405549 & 1.496278 \\
\hline $\mathrm{H}$ & -7.770073 & -0.229847 & 0.194439 & $\mathrm{H}$ & -1.219512 & -4.326263 & 0.287974 \\
\hline $\mathrm{H}$ & -7.627043 & 1.604862 & 0.110591 & $\mathrm{C}$ & 0.62392 & -3.417779 & -0.437903 \\
\hline \multirow[t]{2}{*}{$\mathrm{H}$} & 7.627007 & 1.60487 & -0.110584 & $\mathrm{H}$ & 1.219422 & -4.32635 & -0.286936 \\
\hline & & & & $\mathrm{H}$ & 0.32486 & -3.405945 & -1.495476 \\
\hline \multicolumn{2}{|c|}{$\mathrm{C}(\mathrm{DP}=5)$} & & & $\mathrm{C}$ & 2.832955 & 0.42229 & 0.004499 \\
\hline $\mathrm{C}$ & -10.093127 & 0.336866 & 0.068528 & $\mathrm{C}$ & 3.614673 & 1.64531 & 0.077717 \\
\hline $\mathrm{H}$ & -9.563083 & 1.27894 & -0.032337 & $\mathrm{C}$ & 4.995195 & 1.64808 & 0.015388 \\
\hline $\mathrm{C}$ & -9.293748 & -0.889142 & 0.036127 & $\mathrm{H}$ & 3.384151 & -0.49872 & -0.13699 \\
\hline $\mathrm{C}$ & -7.922001 & -0.880972 & -0.073033 & $\mathrm{C}$ & 5.780578 & 0.425162 & 0.068354 \\
\hline $\mathrm{C}$ & -7.138321 & 0.345392 & -0.010367 & $\mathrm{H}$ & 5.231887 & -0.498087 & 0.205295 \\
\hline $\mathrm{H}$ & -7.688509 & 1.267308 & 0.127618 & $\mathrm{C}$ & 7.138336 & 0.345389 & 0.010185 \\
\hline $\mathrm{C}$ & -10.079974 & -2.188442 & 0.101606 & $\mathrm{H}$ & 7.688508 & 1.267314 & -0.127797 \\
\hline $\mathrm{H}$ & -10.831778 & -2.105539 & 0.896933 & $\mathrm{C}$ & 7.922031 & -0.880968 & 0.072815 \\
\hline $\mathrm{H}$ & -10.653187 & -2.304809 & -0.831765 & $\mathrm{C}$ & 9.293785 & -0.889119 & -0.036294 \\
\hline $\mathrm{C}$ & -7.141273 & -2.178559 & -0.23594 & $\mathrm{C}$ & 7.141293 & -2.178555 & 0.235656 \\
\hline $\mathrm{H}$ & -6.572738 & -2.370738 & 0.687395 & $\mathrm{H}$ & 6.388315 & -2.041369 & 1.021883 \\
\hline $\mathrm{H}$ & -6.388277 & -2.041332 & -1.02214 & $\mathrm{H}$ & 6.572732 & -2.370641 & -0.687681 \\
\hline $\mathrm{C}$ & -9.225861 & -3.436442 & 0.337767 & $\mathrm{C}$ & 10.080013 & -2.188423 & -0.101743 \\
\hline $\mathrm{H}$ & -8.899613 & -3.470652 & 1.386772 & $\mathrm{H}$ & 10.653081 & -2.304852 & 0.83171 \\
\hline $\mathrm{H}$ & -9.826602 & -4.337228 & 0.162899 & $\mathrm{H}$ & 10.831929 & -2.105491 & -0.896957 \\
\hline $\mathrm{C}$ & -8.000595 & -3.400766 & -0.570279 & $\mathrm{C}$ & 8.000592 & -3.40081 & 0.569881 \\
\hline$H$ & -7.401289 & -4.31383 & -0.46963 & $\mathrm{H}$ & 8.326901 & -3.349373 & 1.618152 \\
\hline $\mathrm{H}$ & -8.326957 & -3.349188 & -1.618526 & $\mathrm{H}$ & 7.40128 & -4.313855 & 0.469089 \\
\hline $\mathrm{C}$ & -5.78056 & 0.425188 & -0.06848 & $\mathrm{C}$ & 9.225908 & -3.436388 & -0.338099 \\
\hline $\mathrm{C}$ & -4.995201 & 1.648114 & -0.015463 & $\mathrm{H}$ & 9.826632 & -4.3372 & -0.163301 \\
\hline $\mathrm{C}$ & -3.614675 & 1.645354 & -0.077707 & $\mathrm{H}$ & 8.899727 & -3.470467 & -1.387129 \\
\hline $\mathrm{H}$ & -5.231846 & -0.49805 & -0.2054 & $\mathrm{C}$ & 1.473087 & 0.340343 & 0.046692 \\
\hline $\mathrm{C}$ & -2.832964 & 0.422336 & -0.004413 & $\mathrm{H}$ & 0.921838 & 1.261523 & 0.187124 \\
\hline $\mathrm{H}$ & -3.384172 & -0.498665 & 0.1371 & $\mathrm{C}$ & 10.093152 & 0.336894 & -0.068606 \\
\hline $\mathrm{C}$ & -1.473095 & 0.340372 & -0.046546 & $\mathrm{H}$ & 9.563092 & 1.278957 & 0.032271 \\
\hline $\mathrm{H}$ & -0.921826 & 1.261533 & -0.187017 & $\mathrm{C}$ & 11.432263 & 0.414528 & -0.166222 \\
\hline $\mathrm{C}$ & -0.690862 & -0.881965 & 0.025566 & $\mathrm{H}$ & 11.931418 & 1.378967 & -0.154048 \\
\hline $\mathrm{C}$ & 0.690824 & -0.881986 & -0.025291 & $\mathrm{H}$ & 12.075866 & -0.454694 & -0.256433 \\
\hline $\mathrm{C}$ & -1.473963 & -2.183816 & 0.125326 & $\mathrm{C}$ & 5.776563 & 2.951216 & -0.077935 \\
\hline $\mathrm{H}$ & -2.246133 & -2.07281 & 0.89736 & $\mathrm{H}$ & 6.541803 & 2.848913 & -0.85805 \\
\hline $\mathrm{H}$ & -2.02099 & -2.345151 & -0.816854 & $\mathrm{H}$ & 6.332137 & 3.102665 & 0.860853 \\
\hline $\mathrm{C}$ & 1.473904 & -2.183864 & -0.124851 & $\mathrm{C}$ & 2.830774 & 2.94556 & 0.192849 \\
\hline $\mathrm{H}$ & 2.021016 & -2.344995 & 0.817314 & $\mathrm{H}$ & 2.279775 & 3.115354 & -0.745516 \\
\hline $\mathrm{H}$ & 2.246006 & -2.073023 & -0.896978 & $\mathrm{H}$ & 2.062109 & 2.826586 & 0.967103 \\
\hline & & & & $\mathrm{C}$ & 4.923418 & 4.187974 & -0.370792 \\
\hline
\end{tabular}




$\begin{array}{rrrr}\mathrm{H} & 5.519317 & 5.095166 & -0.213461 \\ \mathrm{H} & 4.617164 & 4.188051 & -1.426365 \\ \mathrm{C} & 3.681397 & 4.176852 & 0.514048 \\ \mathrm{H} & 3.987298 & 4.153419 & 1.569459 \\ \mathrm{H} & 3.083946 & 5.08637 & 0.377157 \\ \mathrm{C} & -2.830774 & 2.945606 & -0.192784 \\ \mathrm{H} & -2.279782 & 3.115359 & 0.745591 \\ \mathrm{H} & -2.062099 & 2.826656 & -0.967034 \\ \mathrm{C} & -5.776578 & 2.951244 & 0.077852 \\ \mathrm{H} & -6.541882 & 2.848903 & 0.857901 \\ \mathrm{H} & -6.332078 & 3.10274 & -0.860973 \\ \mathrm{C} & -3.681384 & 4.176916 & -0.513951 \\ \mathrm{H} & -3.987237 & 4.153546 & -1.569378 \\ \mathrm{H} & -3.083941 & 5.086425 & -0.37698 \\ \mathrm{C} & -4.923451 & 4.187986 & 0.370828 \\ \mathrm{H} & -5.519343 & 5.095186 & 0.213519 \\ \mathrm{H} & -4.617251 & 4.188002 & 1.426417 \\ \mathrm{C} & -11.432232 & 0.414483 & 0.166218 \\ \mathrm{H} & -12.075839 & -0.454733 & 0.256439 \\ \mathrm{H} & -11.931404 & 1.378914 & 0.15409\end{array}$

\begin{tabular}{|c|c|c|c|}
\hline $\mathrm{H}$ & 12.931245 & -3.08739 & -0.747946 \\
\hline $\mathrm{H}$ & 12.491917 & -2.752552 & 0.912703 \\
\hline $\mathrm{C}$ & 15.419477 & -4.322705 & 0.024209 \\
\hline $\mathrm{H}$ & 15.275289 & -4.326047 & -1.065433 \\
\hline $\mathrm{H}$ & 15.924008 & -5.262371 & 0.279964 \\
\hline $\mathrm{C}$ & 14.062561 & -4.213384 & 0.712355 \\
\hline $\mathrm{H}$ & 13.432116 & -5.084209 & 0.495131 \\
\hline $\mathrm{H}$ & 14.208426 & -4.190955 & 1.801536 \\
\hline $\mathrm{C}$ & 12.204314 & -0.250589 & -0.087516 \\
\hline $\mathrm{C}$ & 11.518162 & 1.014914 & -0.282097 \\
\hline $\mathrm{C}$ & 10.142282 & 1.098688 & -0.391207 \\
\hline $\mathrm{H}$ & 11.577625 & -1.128054 & 0.010156 \\
\hline $\mathrm{C}$ & 9.303279 & -0.079268 & -0.528465 \\
\hline $\mathrm{H}$ & 9.818135 & -1.02457 & -0.644374 \\
\hline $\mathrm{C}$ & 7.940758 & -0.099345 & -0.568085 \\
\hline $\mathrm{H}$ & 7.421328 & 0.842635 & -0.443264 \\
\hline $\mathrm{C}$ & 7.117389 & -1.288935 & -0.69954 \\
\hline $\mathrm{C}$ & 5.735637 & -1.245049 & -0.66395 \\
\hline $\mathrm{C}$ & 7.857777 & -2.612107 & -0.841778 \\
\hline $\mathrm{H}$ & 8.651399 & -2.493116 & -1.59042 \\
\hline $\mathrm{H}$ & 8.376088 & -2.836508 & 0.103907 \\
\hline $\mathrm{C}$ & 4.909698 & -2.524067 & -0.631229 \\
\hline $\mathrm{H}$ & 4.365652 & -2.621644 & -1.583773 \\
\hline $\mathrm{H}$ & 4.135217 & -2.424343 & 0.139838 \\
\hline $\mathrm{C}$ & 6.973466 & -3.799463 & -1.229121 \\
\hline $\mathrm{H}$ & 6.691487 & -3.724182 & -2.28876 \\
\hline $\mathrm{H}$ & 7.536414 & -4.733939 & -1.116042 \\
\hline $\mathrm{C}$ & 5.713455 & -3.800467 & -0.370302 \\
\hline $\mathrm{H}$ & 5.089608 & -4.679171 & -0.574445 \\
\hline $\mathrm{H}$ & 5.997331 & -3.852049 & 0.690288 \\
\hline $\mathrm{C}$ & 3.634977 & 0.122137 & -0.630811 \\
\hline $\mathrm{C}$ & 2.884698 & 1.365284 & -0.651018 \\
\hline $\mathrm{C}$ & 1.504856 & 1.399829 & -0.55882 \\
\hline $\mathrm{H}$ & 3.060063 & -0.788613 & -0.523351 \\
\hline $\mathrm{C}$ & 0.690358 & 0.19875 & -0.61776 \\
\hline $\mathrm{H}$ & 1.213821 & -0.73354 & -0.78654 \\
\hline $\mathrm{C}$ & -0.66874 & 0.144308 & -0.522966 \\
\hline $\mathrm{H}$ & -1.196169 & 1.07408 & -0.350483 \\
\hline $\mathrm{C}$ & -1.474272 & -1.0634 & -0.575279 \\
\hline $\mathrm{C}$ & -2.851116 & -1.044584 & -0.44502 \\
\hline $\mathrm{C}$ & -3.617803 & 0.189157 & -0.441134 \\
\hline $\mathrm{H}$ & -3.062457 & 1.10677 & -0.589338 \\
\hline
\end{tabular}




\begin{tabular}{|c|c|c|c|c|c|c|c|}
\hline $\mathrm{C}$ & -0.719324 & -2.374883 & -0.745908 & $\mathrm{C}$ & -17.866665 & 0.293665 & 0.51831 \\
\hline $\mathrm{H}$ & 0.012368 & -2.258259 & -1.555497 & $\mathrm{H}$ & -18.403972 & 1.218833 & 0.68301 \\
\hline $\mathrm{H}$ & -0.126198 & -2.567038 & 0.161995 & $\mathrm{C}$ & -18.657925 & -0.929458 & 0.515675 \\
\hline $\mathrm{C}$ & -3.647348 & -2.337585 & -0.331387 & $\mathrm{C}$ & -20.022367 & -0.928003 & 0.694735 \\
\hline $\mathrm{H}$ & -4.249036 & -2.467796 & -1.244556 & $\mathrm{C}$ & -17.892481 & -2.2339 & 0.336674 \\
\hline $\mathrm{H}$ & -4.37253 & -2.235093 & 0.485878 & $\mathrm{H}$ & -17.183982 & -2.117086 & -0.492935 \\
\hline $\mathrm{C}$ & -1.604596 & -3.588165 & -1.040706 & $\mathrm{H}$ & -17.27433 & -2.409338 & 1.230861 \\
\hline $\mathrm{H}$ & -1.961429 & -3.545508 & -2.079406 & $\mathrm{C}$ & -20.809723 & -2.221454 & 0.826756 \\
\hline $\mathrm{H}$ & -1.01597 & -4.508661 & -0.945073 & $\mathrm{H}$ & -21.429489 & -2.354431 & -0.074048 \\
\hline $\mathrm{C}$ & -2.80148 & -3.59131 & -0.095934 & $\mathrm{H}$ & -21.520262 & -2.118322 & 1.656918 \\
\hline $\mathrm{H}$ & -3.419349 & -4.486828 & -0.234604 & $\mathrm{C}$ & -18.773073 & -3.458929 & 0.075414 \\
\hline $\mathrm{H}$ & -2.443836 & -3.609788 & 0.943202 & $\mathrm{H}$ & -19.153461 & -3.429222 & -0.955327 \\
\hline $\mathrm{C}$ & -4.97225 & 0.285817 & -0.317225 & $\mathrm{H}$ & -18.172811 & -4.372492 & 0.16527 \\
\hline $\mathrm{C}$ & -5.743393 & 1.516448 & -0.315517 & $\mathrm{C}$ & -19.94971 & -3.468306 & 1.04635 \\
\hline $\mathrm{C}$ & -7.118951 & 1.529609 & -0.17006 & $\mathrm{H}$ & -20.562258 & -4.36966 & 0.922939 \\
\hline $\mathrm{H}$ & -5.525084 & -0.63266 & -0.167077 & $\mathrm{H}$ & -19.569857 & -3.480911 & 2.077651 \\
\hline $\mathrm{C}$ & -7.916635 & 0.316015 & -0.19522 & $\mathrm{C}$ & -12.217759 & 0.264198 & 0.070892 \\
\hline $\mathrm{H}$ & -7.384988 & -0.610199 & -0.372403 & $\mathrm{H}$ & -11.671995 & 1.184658 & -0.093575 \\
\hline $\mathrm{C}$ & -9.271837 & 0.245564 & -0.063137 & $\mathrm{C}$ & -20.813262 & 0.30296 & 0.741126 \\
\hline $\mathrm{H}$ & -9.805153 & 1.170778 & 0.115107 & $\mathrm{H}$ & -20.284722 & 1.238988 & 0.588863 \\
\hline $\mathrm{C}$ & -10.065958 & -0.970692 & -0.085697 & $\mathrm{C}$ & -22.144942 & 0.390794 & 0.907605 \\
\hline $\mathrm{C}$ & -11.440314 & -0.96312 & 0.068155 & $\mathrm{H}$ & -22.63951 & 1.357634 & 0.899014 \\
\hline $\mathrm{C}$ & -9.301423 & -2.276837 & -0.253443 & $\mathrm{H}$ & -22.786978 & -0.472227 & 1.052388 \\
\hline $\mathrm{H}$ & -8.585481 & -2.163752 & -1.077434 & $\mathrm{C}$ & -16.489226 & 2.892196 & 0.53058 \\
\hline $\mathrm{H}$ & -8.690399 & -2.451252 & 0.646059 & $\mathrm{H}$ & -17.201526 & 2.78711 & 1.358795 \\
\hline $\mathrm{C}$ & -12.222728 & -2.261629 & 0.20955 & $\mathrm{H}$ & -17.10453 & 3.054495 & -0.368306 \\
\hline $\mathrm{H}$ & -12.837091 & -2.408438 & -0.69261 & $\mathrm{C}$ & -13.569737 & 2.877139 & 0.05601 \\
\hline $\mathrm{H}$ & -12.936234 & -2.155905 & 1.036656 & $\mathrm{H}$ & -12.953764 & 3.033694 & 0.955399 \\
\hline $\mathrm{C}$ & -10.179863 & -3.502202 & -0.517392 & $\mathrm{H}$ & -12.85798 & 2.764262 & -0.771559 \\
\hline $\mathrm{H}$ & -10.553983 & -3.476737 & -1.550562 & $\mathrm{C}$ & -15.612474 & 4.122465 & 0.776678 \\
\hline $\mathrm{H}$ & -9.580945 & -4.415793 & -0.419455 & $\mathrm{H}$ & -16.213611 & 5.033569 & 0.669922 \\
\hline $\mathrm{C}$ & -11.361327 & -3.50441 & 0.446785 & $\mathrm{H}$ & -15.23489 & 4.110017 & 1.808818 \\
\hline $\mathrm{H}$ & -11.972771 & -4.407343 & 0.329359 & $\mathrm{C}$ & -14.434099 & 4.115955 & -0.191347 \\
\hline $\mathrm{H}$ & -10.986901 & -3.50638 & 1.480153 & $\mathrm{H}$ & -14.811766 & 4.10626 & -1.223488 \\
\hline $\mathrm{C}$ & 4.992241 & 0.003097 & -0.683247 & $\mathrm{H}$ & -13.823728 & 5.021061 & -0.08561 \\
\hline $\mathrm{H}$ & 5.571091 & 0.912947 & -0.783002 & $\mathrm{C}$ & -7.879755 & 2.838427 & -0.007564 \\
\hline $\mathrm{C}$ & -13.570724 & 0.351975 & 0.211926 & $\mathrm{H}$ & -8.484368 & 3.014422 & -0.911114 \\
\hline $\mathrm{C}$ & -14.349754 & 1.578759 & 0.212099 & $\mathrm{H}$ & -8.601535 & 2.728427 & 0.811757 \\
\hline $\mathrm{C}$ & -15.72229 & 1.586409 & 0.374791 & $\mathrm{C}$ & -4.954516 & 2.811836 & -0.448485 \\
\hline $\mathrm{H}$ & -14.115386 & -0.568902 & 0.376848 & $\mathrm{H}$ & -4.232878 & 2.703406 & -1.268133 \\
\hline $\mathrm{C}$ & -16.514763 & 0.367009 & 0.375393 & $\mathrm{H}$ & -4.349003 & 2.956176 & 0.460071 \\
\hline $\mathrm{H}$ & -15.97971 & -0.559564 & 0.210384 & $\mathrm{C}$ & -6.998457 & 4.060746 & 0.261447 \\
\hline
\end{tabular}




\begin{tabular}{|c|c|c|c|c|c|c|c|}
\hline $\mathrm{H}$ & -6.632321 & 4.035046 & 1.297466 & $\mathrm{C}(\mathrm{DP}=$ & & & \\
\hline $\mathrm{H}$ & -7.593023 & 4.976559 & 0.157931 & $\mathrm{C}$ & 31.549682 & 0.106223 & -0.714764 \\
\hline $\mathrm{C}$ & -5.809401 & 4.057649 & -0.693481 & $\mathrm{C}$ & 30.737704 & -1.101837 & -0.871037 \\
\hline $\mathrm{H}$ & -5.195657 & 4.958466 & -0.571846 & $\mathrm{C}$ & 29.363765 & -1.089393 & -0.796319 \\
\hline $\mathrm{H}$ & -6.175551 & 4.060153 & -1.729814 & $\mathrm{H}$ & 31.022009 & 1.044025 & -0.570292 \\
\hline $\mathrm{C}$ & 0.758749 & 2.719757 & -0.420015 & $\mathrm{C}$ & 28.612524 & 0.093324 & -0.397667 \\
\hline $\mathrm{H}$ & 0.189605 & 2.906221 & -1.344283 & $\mathrm{H}$ & 29.191458 & 0.969974 & -0.136577 \\
\hline $\mathrm{H}$ & 0.006136 & 2.619054 & 0.372357 & $\mathrm{C}$ & 27.258325 & 0.176835 & -0.286127 \\
\hline $\mathrm{C}$ & 3.698801 & 2.648248 & -0.744452 & $\mathrm{H}$ & 26.68014 & -0.699703 & -0.549951 \\
\hline $\mathrm{H}$ & 4.454901 & 2.529878 & -1.531062 & $\mathrm{C}$ & 26.506674 & 1.358595 & 0.105421 \\
\hline $\mathrm{H}$ & 4.265052 & 2.781856 & 0.190788 & $\mathrm{C}$ & 25.126911 & 1.366649 & 0.182812 \\
\hline $\mathrm{C}$ & 1.647916 & 3.928373 & -0.116255 & C & 24.331903 & 0.155383 & 0.067643 \\
\hline $\mathrm{H}$ & 1.972653 & 3.897277 & 0.933327 & $\mathrm{H}$ & 24.877122 & -0.774898 & -0.031501 \\
\hline $\mathrm{H}$ & 1.072138 & 4.853268 & -0.242663 & C & 32.891001 & 0.180349 & -0.77945 \\
\hline $\mathrm{C}$ & 2.873937 & 3.907093 & -1.023115 & $\mathrm{H}$ & 33.396588 & 1.136009 & -0.676779 \\
\hline $\mathrm{H}$ & 3.495898 & 4.798456 & -0.876171 & $\mathrm{H}$ & 33.529603 & -0.682922 & -0.937435 \\
\hline $\mathrm{H}$ & 2.549821 & 3.915239 & -2.073323 & $\mathrm{C}$ & 27.318914 & 2.612904 & 0.395298 \\
\hline $\mathrm{C}$ & 9.433977 & 2.445732 & -0.407813 & $\mathrm{H}$ & 28.158167 & 2.346513 & 1.050499 \\
\hline $\mathrm{H}$ & 8.99729 & 2.605602 & -1.406026 & $\mathrm{H}$ & 27.77782 & 2.966154 & -0.541528 \\
\hline $\mathrm{H}$ & 8.580674 & 2.405391 & 0.281411 & C & 24.361768 & 2.659276 & 0.430882 \\
\hline $\mathrm{C}$ & 12.387088 & 2.26351 & -0.317299 & $\mathrm{H}$ & 23.908004 & 2.618168 & 1.433477 \\
\hline $\mathrm{H}$ & 13.238348 & 2.083275 & -0.986236 & $\mathrm{H}$ & 23.519428 & 2.711939 & -0.270483 \\
\hline $\mathrm{H}$ & 12.825411 & 2.423555 & 0.680346 & $\mathrm{C}$ & 26.52507 & 3.754072 & 1.035835 \\
\hline $\mathrm{C}$ & 10.31947 & 3.639201 & -0.038789 & $\mathrm{H}$ & 26.326802 & 3.522529 & 2.091858 \\
\hline $\mathrm{H}$ & 10.494692 & 3.651526 & 1.046248 & $\mathrm{H}$ & 27.120422 & 4.675041 & 1.019429 \\
\hline $\mathrm{H}$ & 9.802775 & 4.574431 & -0.28651 & $\mathrm{C}$ & 25.200022 & 3.933438 & 0.301873 \\
\hline $\mathrm{C}$ & 11.658108 & 3.533169 & -0.763307 & $\mathrm{H}$ & 24.637023 & 4.788849 & 0.694619 \\
\hline $\mathrm{H}$ & 12.287743 & 4.410084 & -0.569589 & $\mathrm{H}$ & 25.397928 & 4.143179 & -0.75873 \\
\hline $\mathrm{H}$ & 11.483343 & 3.50094 & -1.847972 & $\mathrm{C}$ & 22.971701 & 0.084463 & 0.121525 \\
\hline $\mathrm{C}$ & 17.962776 & 1.902022 & 0.803132 & $\mathrm{C}$ & 22.177248 & -1.126434 & 0.007196 \\
\hline $\mathrm{H}$ & 17.584514 & 2.073894 & -0.216605 & C & 20.794952 & -1.113509 & 0.04856 \\
\hline $\mathrm{H}$ & 17.073234 & 1.866424 & 1.444618 & $\mathrm{H}$ & 22.42657 & 1.014967 & 0.218 \\
\hline $\mathrm{C}$ & 20.905475 & 1.686289 & 1.01898 & $\mathrm{C}$ & 20.036003 & 0.076869 & 0.390914 \\
\hline $\mathrm{H}$ & 21.306103 & 1.821019 & 2.036157 & $\mathrm{H}$ & 20.607647 & 0.955866 & 0.660905 \\
\hline $\mathrm{H}$ & 21.78107 & 1.501689 & 0.383322 & $\mathrm{C}$ & 18.67697 & 0.171368 & 0.444008 \\
\hline $\mathrm{C}$ & 18.841793 & 3.081235 & 1.22884 & $\mathrm{H}$ & 18.102708 & -0.70471 & 0.169978 \\
\hline $\mathrm{H}$ & 18.966095 & 3.076313 & 2.320891 & $\mathrm{C}$ & 17.91821 & 1.365601 & 0.77188 \\
\hline $\mathrm{H}$ & 18.345801 & 4.024907 & 0.970984 & $\mathrm{C}$ & 16.535803 & 1.377751 & 0.798302 \\
\hline $\mathrm{C}$ & 20.211874 & 2.973128 & 0.565633 & C & 18.720801 & 2.629591 & 1.045124 \\
\hline $\mathrm{H}$ & 20.840778 & 3.839213 & 0.80501 & $\mathrm{H}$ & 19.532255 & 2.388836 & 1.743905 \\
\hline \multirow[t]{2}{*}{$\mathrm{H}$} & 20.087279 & 2.963108 & -0.526353 & $\mathrm{H}$ & 19.217741 & 2.946725 & 0.114872 \\
\hline & & & & $\mathrm{C}$ & 15.763524 & 2.680601 & 0.950085 \\
\hline
\end{tabular}




\begin{tabular}{|c|c|c|c|c|c|c|c|}
\hline $\mathrm{H}$ & 15.256855 & 2.682669 & 1.927732 & $\mathrm{C}$ & 1.462333 & 2.695496 & 0.22106 \\
\hline $\mathrm{H}$ & 14.959252 & 2.7044 & 0.203531 & $\mathrm{H}$ & 2.183 & 2.586138 & 1.041446 \\
\hline $\mathrm{C}$ & 17.902684 & 3.793747 & 1.608687 & $\mathrm{H}$ & 2.068581 & 2.856165 & -0.684236 \\
\hline $\mathrm{H}$ & 17.657435 & 3.600834 & 2.66259 & $\mathrm{C}$ & -1.462312 & 2.69548 & -0.221928 \\
\hline $\mathrm{H}$ & 18.500174 & 4.7132 & 1.585101 & $\mathrm{H}$ & -2.068546 & 2.85616 & 0.683375 \\
\hline $\mathrm{C}$ & 16.611588 & 3.947523 & 0.810307 & $\mathrm{H}$ & -2.182991 & 2.586108 & -1.042302 \\
\hline $\mathrm{H}$ & 16.034617 & 4.818291 & 1.144737 & $\mathrm{C}$ & 0.593978 & 3.929589 & 0.477648 \\
\hline $\mathrm{H}$ & 16.857504 & 4.116803 & -0.247505 & $\mathrm{H}$ & 0.226615 & 3.917751 & 1.513488 \\
\hline $\mathrm{C}$ & 14.386425 & 0.087926 & 0.678008 & $\mathrm{H}$ & 1.198335 & 4.838003 & 0.365903 \\
\hline $\mathrm{C}$ & 13.601671 & -1.132886 & 0.62038 & $\mathrm{C}$ & -0.593969 & 3.929575 & -0.478544 \\
\hline $\mathrm{C}$ & 12.223544 & -1.127972 & 0.499927 & $\mathrm{H}$ & -1.198335 & 4.837985 & -0.366818 \\
\hline $\mathrm{H}$ & 13.839326 & 1.021568 & 0.649937 & $\mathrm{H}$ & -0.226608 & 3.917715 & -1.514384 \\
\hline $\mathrm{C}$ & 11.436845 & 0.091282 & 0.567425 & $\mathrm{C}$ & 15.747191 & 0.159848 & 0.721645 \\
\hline $\mathrm{H}$ & 11.980063 & 1.013532 & 0.729024 & $\mathrm{H}$ & 16.297451 & -0.77279 & 0.741994 \\
\hline $\mathrm{C}$ & 10.078694 & 0.172111 & 0.478177 & $\mathrm{C}$ & -2.828353 & 0.090927 & -0.185759 \\
\hline $\mathrm{H}$ & 9.533937 & -0.748249 & 0.310484 & $\mathrm{C}$ & -3.614372 & -1.130331 & -0.169856 \\
\hline $\mathrm{C}$ & 9.293631 & 1.392966 & 0.531693 & $\mathrm{C}$ & -4.990219 & -1.129916 & -0.313359 \\
\hline $\mathrm{C}$ & 7.915562 & 1.394806 & 0.41292 & $\mathrm{H}$ & -3.368981 & 1.014131 & -0.351347 \\
\hline $\mathrm{C}$ & 7.130872 & 0.172518 & 0.420574 & $\mathrm{C}$ & -5.77469 & 0.092346 & -0.304288 \\
\hline $\mathrm{H}$ & 7.674006 & -0.751791 & 0.571513 & $\mathrm{H}$ & -5.231995 & 1.016517 & -0.151504 \\
\hline $\mathrm{C}$ & 10.070544 & 2.692937 & 0.689697 & $\mathrm{C}$ & -7.130854 & 0.172516 & -0.421068 \\
\hline $\mathrm{H}$ & 10.807921 & 2.568146 & 1.49298 & $\mathrm{H}$ & -7.674005 & -0.751786 & -0.572003 \\
\hline $\mathrm{H}$ & 10.65823 & 2.87169 & -0.22444 & $\mathrm{C}$ & -7.915541 & 1.394805 & -0.413307 \\
\hline $\mathrm{C}$ & 7.138206 & 2.699214 & 0.300383 & $\mathrm{C}$ & -9.293618 & 1.392963 & -0.532009 \\
\hline $\mathrm{H}$ & 6.546335 & 2.842379 & 1.218019 & $\mathrm{C}$ & -7.138185 & 2.699212 & -0.300765 \\
\hline $\mathrm{H}$ & 6.404587 & 2.604584 & -0.51029 & $\mathrm{H}$ & -6.404575 & 2.60457 & 0.509918 \\
\hline $\mathrm{C}$ & 9.206552 & 3.920944 & 0.986936 & $\mathrm{H}$ & -6.546301 & 2.84238 & -1.218391 \\
\hline $\mathrm{H}$ & 8.858185 & 3.888118 & 2.028865 & $\mathrm{C}$ & -10.070534 & 2.692933 & -0.690008 \\
\hline $\mathrm{H}$ & 9.80824 & 4.831961 & 0.882206 & $\mathrm{H}$ & -10.658191 & 2.871713 & 0.224142 \\
\hline $\mathrm{C}$ & 8.001528 & 3.93865 & 0.052524 & $\mathrm{H}$ & -10.807936 & 2.568121 & -1.493265 \\
\hline $\mathrm{H}$ & 7.398983 & 4.844301 & 0.192768 & $\mathrm{C}$ & -8.001503 & 3.938652 & -0.052907 \\
\hline $\mathrm{H}$ & 8.350428 & 3.947335 & -0.989706 & $\mathrm{H}$ & -8.350392 & 3.947355 & 0.989326 \\
\hline $\mathrm{C}$ & 5.774721 & 0.092354 & 0.30366 & $\mathrm{H}$ & -7.398961 & 4.8443 & -0.193172 \\
\hline $\mathrm{C}$ & 4.990245 & -1.129907 & 0.312602 & $\mathrm{C}$ & -9.206544 & 3.920928 & -0.987303 \\
\hline $\mathrm{C}$ & 3.614411 & -1.130309 & 0.168983 & $\mathrm{H}$ & -9.808224 & 4.831951 & -0.882589 \\
\hline $\mathrm{H}$ & 5.23204 & 1.016531 & 0.150862 & $\mathrm{H}$ & -8.858193 & 3.888068 & -2.029236 \\
\hline $\mathrm{C}$ & 2.82839 & 0.090949 & 0.184903 & $\mathrm{C}$ & -1.47312 & 0.17127 & -0.058649 \\
\hline $\mathrm{H}$ & 3.369018 & 1.014148 & 0.350522 & $\mathrm{H}$ & -0.932065 & -0.7516 & 0.107612 \\
\hline $\mathrm{C}$ & 1.473159 & 0.171289 & 0.05778 & $\mathrm{C}$ & -10.078671 & 0.172109 & -0.4784 \\
\hline $\mathrm{H}$ & 0.932113 & -0.751583 & -0.108501 & $\mathrm{H}$ & -9.533887 & -0.74824 & -0.31073 \\
\hline $\mathrm{C}$ & 0.687837 & 1.39302 & 0.07204 & $\mathrm{C}$ & -11.436831 & 0.091273 & -0.567517 \\
\hline $\mathrm{C}$ & -0.687806 & 1.393012 & -0.072902 & $\mathrm{H}$ & -11.980061 & 1.013522 & -0.729081 \\
\hline
\end{tabular}




\begin{tabular}{|c|c|c|c|c|c|c|c|}
\hline $\mathrm{C}$ & -5.765889 & -2.432445 & -0.455766 & $\mathrm{C}$ & 20.795913 & -3.528669 & -0.833211 \\
\hline $\mathrm{H}$ & -6.489718 & -2.32493 & -1.273576 & $\mathrm{H}$ & 21.020529 & -3.320201 & -1.888768 \\
\hline $\mathrm{H}$ & -6.36858 & -2.590592 & 0.452387 & $\mathrm{H}$ & 20.198371 & -4.448168 & -0.811863 \\
\hline $\mathrm{C}$ & -2.84032 & -2.432923 & -0.019207 & $\mathrm{C}$ & 22.102486 & -3.694594 & -0.063774 \\
\hline $\mathrm{H}$ & -2.235581 & -2.595628 & -0.925156 & $\mathrm{H}$ & 22.673204 & -4.558833 & -0.424848 \\
\hline $\mathrm{H}$ & -2.118285 & -2.322236 & 0.79977 & $\mathrm{H}$ & 21.87906 & -3.881607 & 0.996027 \\
\hline $\mathrm{C}$ & -4.898859 & -3.667484 & -0.712079 & $\mathrm{C}$ & 28.550805 & -2.340793 & -1.09994 \\
\hline $\mathrm{H}$ & -5.503381 & -4.575386 & -0.597081 & $\mathrm{H}$ & 28.097879 & -2.705963 & -0.165029 \\
\hline $\mathrm{H}$ & -4.534051 & -3.657813 & -1.748843 & $\mathrm{H}$ & 27.708321 & -2.066555 & -1.747258 \\
\hline $\mathrm{C}$ & -3.708682 & -3.666223 & 0.241309 & $\mathrm{C}$ & 31.508067 & -2.380809 & -1.154988 \\
\hline $\mathrm{H}$ & -4.073695 & -3.65248 & 1.277958 & $\mathrm{H}$ & 31.949432 & -2.314718 & -2.162041 \\
\hline $\mathrm{H}$ & -3.10492 & -4.575065 & 0.129797 & $\mathrm{H}$ & 32.3608 & -2.439051 & -0.466415 \\
\hline $\mathrm{C}$ & 2.840371 & -2.432895 & 0.01821 & $\mathrm{C}$ & 29.343332 & -3.4721 & -1.76069 \\
\hline $\mathrm{H}$ & 2.235575 & -2.595659 & 0.92411 & $\mathrm{H}$ & 29.531616 & -3.226119 & -2.815267 \\
\hline $\mathrm{H}$ & 2.118383 & -2.322162 & -0.800804 & $\mathrm{H}$ & 28.749768 & -4.394389 & -1.751817 \\
\hline $\mathrm{C}$ & 5.765897 & -2.432443 & 0.455023 & $\mathrm{C}$ & 30.676236 & -3.660477 & -1.042348 \\
\hline $\mathrm{H}$ & 6.489635 & -2.324969 & 1.272923 & $\mathrm{H}$ & 31.237107 & -4.506972 & -1.456795 \\
\hline $\mathrm{H}$ & 6.368694 & -2.590539 & -0.453067 & $\mathrm{H}$ & 30.48946 & -3.890391 & 0.016079 \\
\hline $\mathrm{C}$ & 3.708755 & -3.666175 & -0.242323 & $\mathrm{C}$ & -12.22352 & -1.127981 & -0.499935 \\
\hline $\mathrm{H}$ & 4.073857 & -3.652355 & -1.278939 & $\mathrm{C}$ & -13.601662 & -1.132898 & -0.620243 \\
\hline $\mathrm{H}$ & 3.104985 & -4.575026 & -0.130925 & $\mathrm{C}$ & -14.386425 & 0.08791 & -0.677776 \\
\hline $\mathrm{C}$ & 4.898847 & -3.667502 & 0.711171 & $\mathrm{H}$ & -13.839335 & 1.021557 & -0.649688 \\
\hline $\mathrm{H}$ & 5.503381 & -4.575393 & 0.596155 & $\mathrm{C}$ & -15.747192 & 0.159827 & -0.721344 \\
\hline $\mathrm{H}$ & 4.533943 & -3.657914 & 1.747902 & $\mathrm{H}$ & -16.297457 & -0.772808 & -0.741727 \\
\hline $\mathrm{C}$ & 11.446399 & -2.424335 & 0.311926 & $\mathrm{C}$ & -16.535798 & 1.377742 & -0.797892 \\
\hline $\mathrm{H}$ & 10.86454 & -2.626063 & 1.225123 & $\mathrm{C}$ & -17.918207 & 1.365608 & -0.771415 \\
\hline $\mathrm{H}$ & 10.703919 & -2.279511 & -0.482949 & $\mathrm{C}$ & -18.676975 & 0.17137 & -0.443577 \\
\hline $\mathrm{C}$ & 14.381251 & -2.440088 & 0.672243 & $\mathrm{H}$ & -18.102719 & -0.704717 & -0.169567 \\
\hline $\mathrm{H}$ & 15.133351 & -2.371121 & 1.468087 & $\mathrm{C}$ & -15.763511 & 2.680589 & -0.949631 \\
\hline $\mathrm{H}$ & 14.951044 & -2.554724 & -0.263489 & $\mathrm{H}$ & -14.95921 & 2.704324 & -0.203104 \\
\hline $\mathrm{C}$ & 12.306944 & -3.645312 & -0.022418 & $\mathrm{H}$ & -15.256876 & 2.682717 & -1.927296 \\
\hline $\mathrm{H}$ & 12.643985 & -3.588137 & -1.067035 & $\mathrm{C}$ & -18.72079 & 2.629621 & -1.044579 \\
\hline $\mathrm{H}$ & 11.705449 & -4.558147 & 0.066849 & $\mathrm{H}$ & -19.217718 & 2.946713 & -0.114306 \\
\hline $\mathrm{C}$ & 13.52239 & -3.686582 & 0.897391 & $\mathrm{H}$ & -19.532255 & 2.3889 & -1.743361 \\
\hline $\mathrm{H}$ & 14.123872 & -4.587584 & 0.725842 & $\mathrm{C}$ & -16.611556 & 3.947512 & -0.809741 \\
\hline $\mathrm{H}$ & 13.186334 & -3.72303 & 1.943242 & $\mathrm{H}$ & -16.857436 & 4.116722 & 0.248091 \\
\hline $\mathrm{C}$ & 19.988491 & -2.372902 & -0.237416 & $\mathrm{H}$ & -16.034582 & 4.818295 & -1.144129 \\
\hline $\mathrm{H}$ & 19.50428 & -2.705486 & 0.694184 & $\mathrm{C}$ & -17.902676 & 3.793801 & -1.608094 \\
\hline $\mathrm{H}$ & 19.167706 & -2.119841 & -0.920374 & $\mathrm{H}$ & -18.500151 & 4.713262 & -1.584437 \\
\hline $\mathrm{C}$ & 22.945406 & -2.424363 & -0.199914 & $\mathrm{H}$ & -17.657457 & 3.600949 & -2.662015 \\
\hline $\mathrm{H}$ & 23.77348 & -2.463758 & 0.519162 & $\mathrm{C}$ & -20.036008 & 0.076871 & -0.390512 \\
\hline $\mathrm{H}$ & 23.419221 & -2.404234 & -1.19397 & $\mathrm{C}$ & -20.794961 & -1.113516 & -0.048201 \\
\hline
\end{tabular}




\begin{tabular}{|c|c|c|c|c|c|c|c|}
\hline $\mathrm{C}$ & -22.177257 & -1.126456 & -0.006859 & $\mathrm{H}$ & -27.708393 & -2.066531 & 1.747722 \\
\hline $\mathrm{H}$ & -20.607643 & 0.955878 & -0.660488 & $\mathrm{C}$ & -30.676316 & -3.660431 & 1.042798 \\
\hline $\mathrm{C}$ & -22.971728 & 0.084424 & -0.12125 & $\mathrm{H}$ & -31.237206 & -4.506908 & 1.457257 \\
\hline $\mathrm{H}$ & -22.426615 & 1.014925 & -0.217853 & $\mathrm{H}$ & -30.489516 & -3.890381 & -0.015617 \\
\hline $\mathrm{C}$ & -24.331926 & 0.155349 & -0.067295 & $\mathrm{C}$ & -29.343424 & -3.472051 & 1.761163 \\
\hline $\mathrm{H}$ & -24.877146 & -0.774919 & 0.031963 & $\mathrm{H}$ & -29.531723 & -3.226038 & 2.815731 \\
\hline $\mathrm{C}$ & -25.126936 & 1.366608 & -0.182523 & $\mathrm{H}$ & -28.749868 & -4.394344 & 1.752325 \\
\hline $\mathrm{C}$ & -26.506699 & 1.358563 & -0.105112 & $\mathrm{C}$ & -22.945398 & -2.424386 & 0.200323 \\
\hline $\mathrm{C}$ & -24.361798 & 2.659222 & -0.430677 & $\mathrm{H}$ & -23.41916 & -2.404223 & 1.194405 \\
\hline $\mathrm{H}$ & -23.519429 & 2.711905 & 0.270653 & $\mathrm{H}$ & -23.773508 & -2.463821 & -0.518706 \\
\hline $\mathrm{H}$ & -23.908072 & 2.61808 & -1.433288 & $\mathrm{C}$ & -19.988488 & -2.372902 & 0.23777 \\
\hline $\mathrm{C}$ & -27.318944 & 2.612856 & -0.395042 & $\mathrm{H}$ & -19.167686 & -2.119847 & 0.920709 \\
\hline $\mathrm{H}$ & -27.777863 & 2.966133 & 0.541766 & $\mathrm{H}$ & -19.5043 & -2.705479 & -0.693844 \\
\hline $\mathrm{H}$ & -28.158188 & 2.346426 & -1.05024 & $\mathrm{C}$ & -22.102479 & -3.694616 & 0.064185 \\
\hline $\mathrm{C}$ & -25.200046 & 3.933389 & -0.301681 & $\mathrm{H}$ & -21.879079 & -3.881648 & -0.995619 \\
\hline $\mathrm{H}$ & -25.397928 & 4.143151 & 0.758922 & $\mathrm{H}$ & -22.673184 & -4.55885 & 0.42529 \\
\hline $\mathrm{H}$ & -24.637053 & 4.788791 & -0.694456 & $\mathrm{C}$ & -20.795886 & -3.528675 & 0.833585 \\
\hline $\mathrm{C}$ & -26.525107 & 3.754012 & -1.035613 & $\mathrm{H}$ & -20.198336 & -4.448169 & 0.812222 \\
\hline $\mathrm{H}$ & -27.120458 & 4.674981 & -1.019213 & $\mathrm{H}$ & -21.020478 & -3.320207 & 1.889147 \\
\hline $\mathrm{H}$ & -26.326861 & 3.522449 & -2.091637 & $\mathrm{C}$ & -14.38125 & -2.440098 & -0.672015 \\
\hline $\mathrm{C}$ & -28.612555 & 0.09333 & 0.39804 & $\mathrm{H}$ & -14.950949 & -2.554716 & 0.263777 \\
\hline $\mathrm{C}$ & -29.363809 & -1.089367 & 0.796729 & $\mathrm{H}$ & -15.133427 & -2.371142 & -1.467784 \\
\hline $\mathrm{C}$ & -30.737749 & -1.101795 & 0.871426 & $\mathrm{C}$ & -11.446355 & -2.424346 & -0.312015 \\
\hline $\mathrm{H}$ & -29.191482 & 0.969973 & 0.136912 & $\mathrm{H}$ & -10.703782 & -2.279524 & 0.48277 \\
\hline $\mathrm{C}$ & -31.549706 & 0.106274 & 0.715114 & $\mathrm{H}$ & -10.864607 & -2.626082 & -1.225283 \\
\hline $\mathrm{H}$ & -31.022012 & 1.044063 & 0.570627 & $\mathrm{C}$ & -13.522415 & -3.686599 & -0.897233 \\
\hline $\mathrm{C}$ & -32.891023 & 0.180432 & 0.779791 & $\mathrm{H}$ & -13.186473 & -3.723064 & -1.943121 \\
\hline $\mathrm{H}$ & -33.529647 & -0.682819 & 0.937792 & $\mathrm{H}$ & -14.12388 & -4.587597 & -0.725607 \\
\hline $\mathrm{C}$ & -27.258356 & 0.176826 & 0.286498 & $\mathrm{C}$ & -12.306866 & -3.645316 & 0.022441 \\
\hline $\mathrm{H}$ & -26.680177 & -0.699705 & 0.550357 & $\mathrm{H}$ & -11.705384 & -4.558155 & -0.066873 \\
\hline $\mathrm{C}$ & -31.508135 & -2.380752 & 1.155381 & $\mathrm{H}$ & -12.643792 & -3.588119 & 1.067094 \\
\hline $\mathrm{H}$ & -32.360845 & -2.438998 & 0.46678 & $\mathrm{H}$ & -33.396587 & 1.136102 & 0.677095 \\
\hline $\mathrm{H}$ & -31.949535 & -2.314629 & 2.162416 & & & & \\
\hline $\mathrm{C}$ & -28.55087 & -2.340768 & 1.100396 & & & & \\
\hline $\mathrm{H}$ & -28.097936 & -2.705969 & 0.1655 & & & & \\
\hline
\end{tabular}


$\mathbf{A}(\mathrm{DP}=1)$

$\begin{array}{rrr}100.7524 & 132.7443 & 160.5307 \\ 175.559 & 225.1347 & 285.442 \\ 377.4055 & 438.3976 & 590.0505 \\ 623.386 & 671.2238 & 724.0516 \\ 743.0399 & 830.0584 & 922.2296 \\ 922.4425 & 925.7066 & 979.2832 \\ 1022.7341 & 1028.159 & 1030.9572 \\ 1053.785 & 1063.16 & 1102.63 \\ 1184.0692 & 1228.273 & 1233.2283 \\ 1287.3021 & 1327.484 & 1335.247 \\ 1379.6207 & 1450.355 & 1481.8049 \\ 1498.8418 & 1516.14 & 1641.1861 \\ 1695.7849 & 1710.7 & 3046.9622 \\ 3052.1342 & 3083.522 & 3098.2682 \\ 3149.4827 & 3155.441 & 3168.8312 \\ 3169.0457 & 3250.699 & 3250.8658\end{array}$

$\mathbf{A}(\mathrm{DP}=3)$

$\begin{array}{rrr}18.1705 & 23.6707 & 26.2059 \\ 63.0111 & 65.8129 & 77.242 \\ 98.8802 & 116.2131 & 137.2212 \\ 160.6695 & 165.9367 & 171.7718 \\ 202.2611 & 203.4697 & 203.5918 \\ 207.1931 & 233.143 & 240.8237 \\ 320.6197 & 325.4926 & 326.3928 \\ 381.4932 & 384.3177 & 477.8127 \\ 525.3224 & 538.4173 & 567.785 \\ 582.0572 & 593.499 & 642.4892 \\ 655.08 & 702.6922 & 707.6147 \\ 709.84 & 764.4549 & 808.7188 \\ 811.2224 & 822.2438 & 823.5377 \\ 835.4374 & 882.9099 & 887.5963 \\ 916.7888 & 916.8139 & 930.4692 \\ 931.0708 & 936.302 & 982.6529 \\ 986.2968 & 987.5612 & 990.1916 \\ 995.7841 & 1023.4119 & 1023.4736 \\ 1041.6708 & 1042.1925 & 1057.5275 \\ 1060.3757 & 1061.0335 & 1098.997 \\ 1101.2526 & 1102.8615 & 1185.4919 \\ 1185.7312 & 1187.4861 & 1221.4827\end{array}$

$\begin{array}{rrr}1227.2615 & 1228.0486 & 1235.0973 \\ 1235.6237 & 1237.9526 & 1259.3958 \\ 1288.06 & 1290.8567 & 1309.9858 \\ 1317.9059 & 1321.9186 & 1323.6539 \\ 1330.9842 & 1332.2936 & 1387.393 \\ 1389.7596 & 1404.2223 & 1464.805 \\ 1472.1198 & 1497.5071 & 1498.05 \\ 1498.0997 & 1511.2696 & 1514.6284 \\ 1514.8959 & 1584.6596 & 1620.3696 \\ 1630.5814 & 1665.1619 & 1668.2253 \\ 1691.314 & 1698.0589 & 3045.0972 \\ 3045.2655 & 3045.8542 & 3050.5833 \\ 3051.4802 & 3051.9694 & 3081.5358 \\ 3081.5465 & 3081.8447 & 3096.7235 \\ 3097.5117 & 3097.6455 & 3149.0852 \\ 3149.9198 & 3151.7125 & 3154.3964 \\ 3158.4845 & 3160.4319 & 3169.2628 \\ 3169.2787 & 3251.5683 & 3251.5743\end{array}$

$\mathbf{A}(\mathrm{DP}=5)$

\begin{tabular}{rrr}
6.8313 & 9.944 & 12.6801 \\
20.3777 & 26.3365 & 26.4394 \\
41.6445 & 64.292 & 72.2609 \\
74.8439 & 77.5984 & 78.7601 \\
86.9955 & 120.9548 & 130.2815 \\
143.2283 & 157.0752 & 157.7808 \\
163.7819 & 166.9111 & 183.141 \\
202.32 & 202.6482 & 202.7628 \\
203.136 & 206.16 & 208.7332 \\
224.1996 & 240.8 & 241.2341 \\
257.4216 & 300.2232 & 326.5764 \\
326.6148 & 356.3196 & 359.1646 \\
377.7035 & 388.4127 & 410.2801 \\
485.2195 & 504.5428 & 533.3072 \\
541.8821 & 549.3403 & 550.0527 \\
576.1826 & 579.0317 & 586.1224 \\
611.9355 & 646.9948 & 649.4371 \\
692.6206 & 695.7176 & 708.2957 \\
708.3607 & 724.8091 & 771.7681 \\
796.6929 & 806.408 & 811.5061 \\
\hline
\end{tabular}




\begin{tabular}{|c|c|c|}
\hline 815.913 & 821.9732 & 822.6067 \\
\hline 822.6697 & 834.927 & 839.6356 \\
\hline 881.3609 & 883.5835 & 886.5155 \\
\hline 889.037 & 916.317 & 916.3251 \\
\hline 930.8422 & 930.8432 & 936.2341 \\
\hline 936.5509 & 937.3236 & 981.8207 \\
\hline 981.9556 & 984.6519 & 985.2917 \\
\hline 987.731 & 988.6632 & 990.2191 \\
\hline 996.439 & 1000.4748 & 1023.2785 \\
\hline 1023.2839 & 1041.8998 & 1041.9749 \\
\hline 1056.0129 & 1057.3504 & 1058.6278 \\
\hline 1060.5552 & 1060.6263 & 1098.4083 \\
\hline 1099.5023 & 1100.9645 & 1102.2534 \\
\hline 1102.9777 & 1185.7244 & 1185.7367 \\
\hline 1187.6616 & 1188.0778 & 1188.7086 \\
\hline 1215.0413 & 1223.0808 & 1226.2551 \\
\hline 1227.6807 & 1227.7287 & 1235.465 \\
\hline 1235.48 & 1238.3901 & 1238.6569 \\
\hline 1239.0053 & 1249.3231 & 1267.8379 \\
\hline 1285.2007 & 1287.5051 & 1291.4299 \\
\hline 1309.8894 & 1311.8736 & 1315.0227 \\
\hline 1316.0225 & 1316.6983 & 1319.5392 \\
\hline 1320.1981 & 1330.1503 & 1331.21 \\
\hline 1335.4 & 1388.5948 & 1388.8364 \\
\hline 1400.7124 & 1402.7882 & 1420.4866 \\
\hline 1466.9134 & 1470.0427 & 1497.121 \\
\hline 1497.2646 & 1497.3024 & 1497.9883 \\
\hline 1498.0141 & 1504.3184 & 1512.0445 \\
\hline 1513.702 & 1514.6359 & 1514.6615 \\
\hline 1555.5491 & 1583.9949 & 1595.6121 \\
\hline 1625.4228 & 1625.9656 & 1649.8417 \\
\hline 1657.9965 & 1670.3464 & 1670.3496 \\
\hline 1693.3104 & 1694.528 & 3044.9586 \\
\hline 3045.0371 & 3045.7036 & 3045.8443 \\
\hline 3045.8589 & 3050.3938 & 3050.4081 \\
\hline 3050.8198 & 3051.6943 & 3051.9086 \\
\hline 3081.3825 & 3081.3838 & 3081.7386 \\
\hline 3081.7398 & 3081.7584 & 3096.6197 \\
\hline 3096.6986 & 3096.8802 & 3097.595 \\
\hline 3097.6208 & 3148.8956 & 3149.2954 \\
\hline 3150.0197 & 3151.0843 & 3152.6379 \\
\hline 3154.0823 & 3157.0746 & 3158.729 \\
\hline
\end{tabular}

$\begin{array}{lll}3159.9727 & 3160.7579 & 3169.2833 \\ 3169.2886 & 3251.6165 & 3251.6187\end{array}$

$\mathbf{A}(\mathrm{DP}=10)$

$\begin{array}{lll}-5.4134 & 2.0322 & 2.2362\end{array}$

$\begin{array}{lll}5.0137 & 7.5194 & 10.0105\end{array}$

$12.6034 \quad 14.9353 \quad 16.1646$

$19.2105 \quad 23.6904 \quad 24.2736$

$\begin{array}{lll}25.2137 & 31.8053 & 33.36\end{array}$

$\begin{array}{lll}43.4632 & 47.2093 & 64.423\end{array}$

$\begin{array}{lll}65.8409 & 68.9795 & 72.4951\end{array}$

$\begin{array}{lll}75.9012 & 76.3217 & 78.175\end{array}$

$\begin{array}{lll}79.9922 & 87.144 & 88.7828\end{array}$

$\begin{array}{lll}92.498 & 111.0417 & 124.0798\end{array}$

$125.5961 \quad 135.8156 \quad 143.879$

$154.6846 \quad 155.1461 \quad 158.1855$

$161.6648 \quad 162.0517 \quad 162.9983$

$\begin{array}{lll}164.9589 & 167.2389 & 167.2879\end{array}$

$\begin{array}{lll}184.5236 & 197.4927 & 199.2695\end{array}$

$202.5284 \quad 202.53 \quad 202.6745$

$202.7182 \quad 205.0186 \quad 205.6721$

$205.9659 \quad 207.2678 \quad 213.5943$

$217.7165 \quad 221.4326 \quad 228.0282$

$233.7759 \quad 236.9666 \quad 246.3596$

$254.8117 \quad 261.4404 \quad 264.4731$

$266.1525 \quad 298.0518 \quad 326.6137$

$326.6144 \quad 330.5899 \quad 357.6196$

$359.1769 \quad 359.2588 \quad 363.6509$

$\begin{array}{lll}371.189 & 380.5874 & 382.091\end{array}$

$392.0281 \quad 396.5086 \quad 413.0638$

$429.0169 \quad 490.0567 \quad 493.4815$

$515.3692 \quad 529.6113 \quad 531.151$

$535.005 \quad 536.7594 \quad 540.0474$

$546.8916 \quad 551.6708 \quad 554.3066$

$559.0942 \quad 561.6109 \quad 572.3027$

$577.8728 \quad 578.324 \quad 588.313$

$601.7198 \quad 611.8107 \quad 617.7083$

$648.0459 \quad 648.0511 \quad 688.4965$

$\begin{array}{lll}688.7393 & 692.7335 & 693.6721\end{array}$

$696.2283 \quad 708.1816 \quad 708.1828$

$\begin{array}{lll}709.4688 & 728.1982 & 748.8668\end{array}$ 


\begin{tabular}{|c|c|c|c|c|c|}
\hline 777.5277 & 786.5 & 803.8012 & 1310.5099 & 1311.5218 & 1313.0238 \\
\hline 804.2217 & 805.8073 & 807.9427 & 1313.126 & 1313.4134 & 1313.4773 \\
\hline 810.2627 & 812.5577 & 814.441 & 1314.8789 & 1315.43 & 1315.9412 \\
\hline 815.8378 & 816.1998 & 816.4957 & 1316.204 & 1316.4712 & 1319.0457 \\
\hline 822.5717 & 822.5745 & 825.481 & 1319.6576 & 1326.0907 & 1330.4109 \\
\hline 832.0989 & 836.6449 & 838.9026 & 1331.1769 & 1335.0057 & 1339.2207 \\
\hline 839.2988 & 839.9479 & 880.7035 & 1388.7692 & 1388.7808 & 1401.3276 \\
\hline 881.3221 & 882.3126 & 883.5928 & 1401.5347 & 1402.341 & 1402.8557 \\
\hline 885.2179 & 886.5601 & 887.9428 & 1404.6454 & 1410.9963 & 1421.7915 \\
\hline 889.0652 & 889.8206 & 915.8953 & 1435.6522 & 1467.3774 & 1468.4375 \\
\hline 915.8986 & 930.8168 & 930.8186 & 1485.8736 & 1496.8844 & 1496.9407 \\
\hline 936.2755 & 936.3376 & 936.675 & 1496.9931 & 1497.0218 & 1497.0697 \\
\hline 936.8316 & 937.1869 & 937.4719 & 1497.1092 & 1497.2664 & 1497.2711 \\
\hline 937.5186 & 937.5616 & 979.031 & 1497.9826 & 1497.9839 & 1502.7425 \\
\hline 980.0362 & 981.1756 & 981.2874 & 1509.2473 & 1511.7278 & 1512.8332 \\
\hline 982.2181 & 982.8941 & 983.8001 & 1513.3912 & 1513.6835 & 1513.7917 \\
\hline 984.9887 & 985.2857 & 985.6964 & 1514.6063 & 1514.6087 & 1529.8353 \\
\hline 988.1616 & 988.1767 & 988.3007 & 1549.1501 & 1564.1211 & 1574.4143 \\
\hline 991.8563 & 995.3816 & 998.1551 & 1578.3345 & 1595.7855 & 1597.2744 \\
\hline 1000.0217 & 1000.9574 & 1001.2199 & 1613.4293 & 1618.7145 & 1628.4459 \\
\hline 1022.8773 & 1022.8795 & 1041.6542 & 1638.4078 & 1641.83 & 1651.657 \\
\hline 1041.6737 & 1055.174 & 1055.6182 & 1655.2546 & 1660.1611 & 1665.5291 \\
\hline 1056.1755 & 1056.797 & 1057.4576 & 1668.9141 & 1670.9955 & 1674.3387 \\
\hline 1058.0653 & 1058.5712 & 1058.8463 & 1693.5297 & 1693.5303 & 3044.9086 \\
\hline 1060.5422 & 1060.5427 & 1098.2384 & 3044.9311 & 3045.6113 & 3045.6149 \\
\hline 1098.5262 & 1099.0297 & 1099.6873 & 3045.6958 & 3045.7241 & 3045.8192 \\
\hline 1100.4551 & 1101.1772 & 1101.8875 & 3045.8272 & 3045.9134 & 3045.9179 \\
\hline 1102.4729 & 1102.8907 & 1103.1274 & 3050.2291 & 3050.2321 & 3050.3233 \\
\hline 1185.7538 & 1185.7552 & 1187.9701 & 3050.338 & 3050.4652 & 3050.5043 \\
\hline 1187.9886 & 1188.3675 & 1188.612 & 3050.654 & 3051.0293 & 3051.6943 \\
\hline 1188.8988 & 1189.1632 & 1189.3922 & 3051.7652 & 3081.2853 & 3081.2909 \\
\hline 1189.5637 & 1210.3925 & 1214.6077 & 3081.6125 & 3081.6174 & 3081.6413 \\
\hline 1219.2416 & 1222.7489 & 1224.9316 & 3081.6424 & 3081.6593 & 3081.6602 \\
\hline 1225.9344 & 1226.115 & 1226.602 & 3081.6787 & 3081.6811 & 3096.5454 \\
\hline 1227.7009 & 1227.7093 & 1235.5216 & 3096.5474 & 3096.5689 & 3096.577 \\
\hline 1235.5321 & 1238.6708 & 1238.6794 & 3096.6323 & 3096.7123 & 3096.8178 \\
\hline 1239.2444 & 1239.2494 & 1239.384 & 3096.9388 & 3097.5224 & 3097.5294 \\
\hline 1239.5957 & 1239.6572 & 1239.7688 & 3148.7019 & 3148.852 & 3149.0636 \\
\hline 1245.7178 & 1249.7351 & 1257.4586 & 3149.3604 & 3149.7576 & 3150.381 \\
\hline 1267.4079 & 1268.868 & 1281.3115 & 3150.9263 & 3151.6335 & 3152.4225 \\
\hline 1283.0785 & 1289.4017 & 1290.9734 & 3153.294 & 3153.7981 & 3155.9263 \\
\hline 1292.1707 & 1309.926 & 1309.9631 & 3156.809 & 3157.6511 & 3158.4587 \\
\hline
\end{tabular}




\begin{tabular}{|c|c|c|c|c|c|}
\hline \multirow{2}{*}{$\begin{array}{l}3159.0325 \\
3160.6347 \\
3169.1721\end{array}$} & 3159.7432 & 3160.2563 & 369.7799 & 376.0007 & 376.6387 \\
\hline & 3160.8585 & 3169.1664 & 383.9918 & 387.9865 & 393.2363 \\
\hline \multirow{2}{*}{3169.1721} & 3251.5509 & 3251.5568 & 393.8917 & 403.6093 & 414.6478 \\
\hline & & & 425.4194 & 434.1846 & 491.5837 \\
\hline $\mathbf{A}(\mathrm{DP}=15)$ & & & 492.3742 & 506.6286 & 518.3525 \\
\hline 2.3451 & 2.7833 & 4.6973 & 527.3233 & 530.9949 & 531.2885 \\
\hline 4.9785 & 6.4011 & 80394 & 533.2274 & 534.3707 & 537.974 \\
\hline 8.3294 & 11.6125 & 12.7372 & 542.2281 & 543.7709 & 543.9337 \\
\hline 13.7306 & 15.5966 & 16.0999 & 550.9109 & 551.0437 & 556.2238 \\
\hline 18.1393 & 19.6661 & 19.9238 & 558.19 & 559.0123 & 564.2417 \\
\hline 22.5249 & 22.8125 & 22.8183 & 564.2492 & 568.0964 & 578.7859 \\
\hline 24.0837 & 27.9464 & 27.9617 & 578.8056 & 579.3837 & 589.3972 \\
\hline 30.6566 & 36.8674 & 40.7705 & 598.4627 & 606.1024 & 612.234 \\
\hline 43.1387 & 48.554 & 49.1012 & 616.6603 & 619.0437 & 648.1966 \\
\hline 67.0117 & 68.3388 & 68.9272 & 648.2047 & 686.9311 & 687.307 \\
\hline 71.3349 & 73.4648 & 75.8517 & 689.8893 & 690.01 & 692.6812 \\
\hline 77.9267 & 78.0658 & 79.7262 & 693.4689 & 693.8186 & 696.2037 \\
\hline 80.9458 & 86.0226 & 86.8086 & 704.6927 & 708.5121 & 708.5135 \\
\hline 90.6691 & 92.7802 & 94.6916 & 716.2532 & 729.4239 & 743.3158 \\
\hline 97.0502 & 105.2027 & 116.9166 & 756.6831 & 779.6669 & 783.5391 \\
\hline 125.6095 & 125.9232 & 133.9751 & 796.3238 & 804.4209 & 805.7601 \\
\hline 139.7526 & 147.3212 & 153.0561 & 805.9521 & 806.8239 & 808.2221 \\
\hline 154.9344 & 157.2526 & 159.6156 & 809.5442 & 811.3361 & 812.4756 \\
\hline 161.3673 & 162.0398 & 164.3124 & 814.166 & 814.2599 & 815.3116 \\
\hline 166.7406 & 166.7514 & 169.0024 & 816.3857 & 816.8715 & 818.0514 \\
\hline 169.7366 & 170.4875 & 170.9383 & 818.0729 & 821.1058 & 824.761 \\
\hline 171.1942 & 186.3984 & 195.5861 & 824.7615 & 826.7631 & 831.2428 \\
\hline 198.6566 & 200.3654 & 201.3613 & 834.6506 & 837.0895 & 838.0277 \\
\hline 202.9186 & 203.3149 & 204.141 & 838.8376 & 839.2943 & 839.8073 \\
\hline 204.1673 & 205.3144 & 205.8357 & 839.9051 & 880.0256 & 880.6354 \\
\hline 206.0878 & 206.4793 & 206.6422 & 881.0787 & 881.7041 & 882.4261 \\
\hline 207.6683 & 210.0691 & 217.4636 & 883.3462 & 884.3861 & 885.4588 \\
\hline 219.4648 & 220.821 & 222.7868 & 886.3492 & 887.2158 & 888.0899 \\
\hline 225.7974 & 230.2419 & 231.4271 & 888.7576 & 889.3849 & 889.5895 \\
\hline 235.2545 & 241.4134 & 247.6286 & 916.4962 & 916.4973 & 931.1599 \\
\hline 252.6052 & 253.0375 & 257.4937 & 931.1608 & 935.8074 & 936.3142 \\
\hline 261.2319 & 264.2407 & 266.0955 & 936.4871 & 936.618 & 936.6815 \\
\hline 274.0342 & 297.9744 & 320.4997 & 937.159 & 937.2408 & 937.3064 \\
\hline 327.1147 & 327.118 & 341.8126 & 937.4423 & 937.6987 & 937.8871 \\
\hline 357.1201 & 357.7496 & 359.0435 & 938.0464 & 938.23 & 977.2411 \\
\hline 360.6972 & 361.6862 & 364.8518 & 978.0312 & 978.5776 & 979.3986 \\
\hline & & & 980.0328 & 980.7665 & 981.5552 \\
\hline
\end{tabular}




\begin{tabular}{|c|c|c|c|c|c|}
\hline 981.624 & 982.1801 & 982.8823 & 1312.8659 & 1313.584 & 1313.9131 \\
\hline 983.1117 & 983.5692 & 984.2781 & 1314.6788 & 1315.0138 & 1315.0292 \\
\hline 984.5742 & 984.6184 & 986.131 & 1315.2363 & 1315.2854 & 1315.836 \\
\hline 987.6799 & 987.6928 & 988.2849 & 1316.03 & 1316.7917 & 1319.2751 \\
\hline 990.7216 & 993.2108 & 995.7401 & 1319.6838 & 1321.9477 & 1327.8483 \\
\hline 997.4342 & 999.2965 & 1000.3359 & 1331.2247 & 1331.7877 & 1333.6843 \\
\hline 1001.0861 & 1001.3639 & 1001.5858 & 1336.8007 & 1338.843 & 1388.9548 \\
\hline 1001.6703 & 1023.1149 & 1023.1155 & 1388.9582 & 1401.283 & 1401.3034 \\
\hline 1042.299 & 1042.3056 & 1055.8008 & 1402.0839 & 1402.2458 & 1402.6401 \\
\hline 1056.1729 & 1056.4647 & 1056.7948 & 1402.9789 & 1403.4129 & 1405.2461 \\
\hline 1057.1682 & 1057.5656 & 1058.0124 & 1409.1431 & 1414.8403 & 1422.3081 \\
\hline 1058.3708 & 1058.7447 & 1059.1253 & 1431.0154 & 1439.5154 & 1468.0163 \\
\hline 1059.3284 & 1059.734 & 1059.7516 & 1468.6495 & 1477.2531 & 1492.0397 \\
\hline 1061.5645 & 1061.5646 & 1100.7266 & 1497.6152 & 1497.8132 & 1497.8201 \\
\hline 1101.1499 & 1101.3445 & 1101.6084 & 1497.9038 & 1497.9467 & 1498.0394 \\
\hline 1101.9736 & 1102.42 & 1102.8081 & 1498.0513 & 1498.1453 & 1498.156 \\
\hline 1103.4263 & 1103.8551 & 1104.3171 & 1498.2796 & 1498.2849 & 1498.4922 \\
\hline 1104.7133 & 1105.1475 & 1105.3085 & 1498.4941 & 1499.2944 & 1499.2948 \\
\hline 1105.7605 & 1105.7794 & 1188.4699 & 1502.7132 & 1508.2355 & 1510.944 \\
\hline 1188.4703 & 1190.1454 & 1190.6921 & 1512.4481 & 1513.1755 & 1513.8233 \\
\hline 1190.7389 & 1190.804 & 1190.8779 & 1514.0923 & 1514.344 & 1514.3948 \\
\hline 1191.2848 & 1191.3095 & 1191.6217 & 1514.6953 & 1514.6962 & 1515.6724 \\
\hline 1191.6543 & 1191.9115 & 1191.9135 & 1515.6731 & 1525.682 & 1533.4099 \\
\hline 1192.1817 & 1192.1827 & 1209.984 & 1547.1765 & 1559.6422 & 1564.173 \\
\hline 1212.4543 & 1215.5983 & 1219.102 & 1566.9088 & 1575.6146 & 1581.7609 \\
\hline 1222.2252 & 1224.6714 & 1226.4197 & 1587.4204 & 1596.1611 & 1600.9465 \\
\hline 1227.1963 & 1227.63 & 1228.3895 & 1607.814 & 1615.6268 & 1619.8199 \\
\hline 1228.6097 & 1228.9039 & 1229.0143 & 1628.885 & 1630.0926 & 1638.0157 \\
\hline 1230.146 & 1230.1475 & 1238.165 & 1642.3375 & 1645.9949 & 1652.7586 \\
\hline 1238.1687 & 1241.4671 & 1241.4676 & 1652.7851 & 1658.3131 & 1660.6711 \\
\hline 1241.765 & 1241.8336 & 1241.9783 & 1662.2781 & 1666.538 & 1669.0738 \\
\hline 1242.0871 & 1242.1353 & 1242.1464 & 1670.8624 & 1673.4218 & 1675.1608 \\
\hline 1242.297 & 1242.3576 & 1242.3836 & 1693.8482 & 1693.8491 & 3045.1142 \\
\hline 1242.566 & 1242.5725 & 1246.2977 & 3045.1236 & 3045.4783 & 3045.6127 \\
\hline 1247.9801 & 1250.7349 & 1255.1614 & 3045.7237 & 3045.732 & 3045.7487 \\
\hline 1259.5218 & 1261.3957 & 1269.271 & 3045.7773 & 3045.843 & 3045.8878 \\
\hline 1271.6106 & 1278.4024 & 1281.999 & 3045.9043 & 3045.9706 & 3046.002 \\
\hline 1285.4115 & 1288.7533 & 1290.7905 & 3046.0542 & 3046.0653 & 3050.1451 \\
\hline 1292.406 & 1292.6652 & 1308.9846 & 3050.283 & 3050.3807 & 3050.4411 \\
\hline 1309.4458 & 1309.6432 & 1309.8661 & 3050.4565 & 3050.4741 & 3050.5405 \\
\hline 1309.899 & 1310.3285 & 1311.4506 & 3050.5959 & 3050.6358 & 3050.6552 \\
\hline 1312.4957 & 1312.7475 & 1312.787 & 3050.7905 & 3051.0148 & 3051.2803 \\
\hline
\end{tabular}




\begin{tabular}{|c|c|c|c|c|c|}
\hline 3051.8597 & 3051.8893 & 3081.358 & 3157.7661 & 3169.0524 & 3173.857 \\
\hline 3081.3829 & 3081.423 & 3081.4233 & 3176.9368 & 3251.131 & 3251 \\
\hline 3081.5715 & 3081.5726 & 3081.6758 & & & \\
\hline 3081.676 & 3081.7028 & 3081.7028 & $\mathbf{B}(\mathrm{DP}=3)$ & & \\
\hline 3081.7479 & 3081.7851 & 3081.7875 & 17.4817 & 20.2983 & 30.4483 \\
\hline 3081.8075 & 3081.81 & 3096.4379 & 61.8116 & 62.5145 & 76.9978 \\
\hline 3096.4854 & 3096.6159 & 3096.6177 & 118.0947 & 121.9632 & 126.3778 \\
\hline 3096.6679 & 3096.6682 & 3096.7762 & 134.0831 & 140.2491 & 149.7433 \\
\hline 3096.7873 & 3096.8193 & 3096.8889 & 150.4381 & 171.6044 & 194.1995 \\
\hline 3096.9411 & 3097.0294 & 3097.0692 & 198.1569 & 202.3214 & 209.5806 \\
\hline 3097.643 & 3097.6441 & 3148.8417 & 220.5225 & 227.3961 & 263.1227 \\
\hline 3148.8621 & 3149.0094 & 3149.1101 & 329.8619 & 333.1533 & 340.3864 \\
\hline 3149.2825 & 3149.4645 & 3149.6066 & 398.832 & 420.4752 & 491.082 \\
\hline 3150.7119 & 3150.8343 & 3151.2121 & 504.6283 & 533.5861 & 533.8505 \\
\hline 3151.6085 & 3152.0786 & 3152.5855 & 547.9002 & 590.1584 & 591.6823 \\
\hline 3153.1187 & 3153.5422 & 3153.8276 & 594.8179 & 623.1835 & 638.4337 \\
\hline 3155.9989 & 3156.4188 & 3157.0073 & 641.0223 & 682.3478 & 714.7931 \\
\hline 3157.5257 & 3158.0632 & 3158.5725 & 721.935 & 745.7204 & 788.6098 \\
\hline 3158.8292 & 3159.2161 & 3160.0902 & 803.7131 & 867.0304 & 867.4382 \\
\hline 3160.2926 & 3160.6278 & 3160.8256 & 870.9721 & 875.2381 & 884.0718 \\
\hline 3161.015 & 3161.3102 & 3169.0717 & 908.6351 & 909.0635 & 912.426 \\
\hline 3169.0728 & 3250.9882 & 3250.9889 & 915.6887 & 915.9391 & 939.2283 \\
\hline & & & 939.5378 & 942.4635 & 972.7297 \\
\hline $\mathbf{B}(\mathrm{DP}=1)$ & & & 974.3071 & 984.4341 & 985.9456 \\
\hline 106.4217 & 140.678 & 146.3001 & 996.8335 & 1020.435 & 1020.534 \\
\hline 162.9512 & 184.5578 & 250.201 & 1026.5933 & 1027.968 & 1043.388 \\
\hline 300.4331 & 402.7884 & 462.1849 & 1055.3196 & 1055.542 & 1068.066 \\
\hline 546.8683 & 578.8831 & 602.585 & 1070.9317 & 1072.738 & 1163.683 \\
\hline 677.1477 & 728.3269 & 746.843 & 1164.678 & 1165.043 & 1221.448 \\
\hline 866.4944 & 909.6433 & 918.6054 & 1226.1704 & 1233.625 & 1235.789 \\
\hline 920.6598 & 936.8824 & 980.5863 & 1244.0174 & 1246.522 & 1253.735 \\
\hline 1016.8262 & 1021.1218 & 1028.284 & 1259.4579 & 1265.198 & 1299.687 \\
\hline 1036.7372 & 1064.5251 & 1072.409 & 1299.9672 & 1303.123 & 1312.962 \\
\hline 1165.7355 & 1232.834 & 1240.268 & 1327.2926 & 1335.962 & 1337.203 \\
\hline 1256.7177 & 1296.4836 & 1331.453 & 1342.5789 & 1344.472 & 1355.207 \\
\hline 1343.8551 & 1353.9175 & 1366.37 & 1356.0872 & 1356.688 & 1361.644 \\
\hline 1403.2018 & 1462.8663 & 1484.638 & 1367.3521 & 1369.596 & 1409.245 \\
\hline 1514.615 & 1517.1902 & 1538.116 & 1409.7334 & 1418.131 & 1473.607 \\
\hline 1632.3751 & 1696.0578 & 1696.324 & 1476.6524 & 1513.168 & 1514.228 \\
\hline 3017.618 & 3017.7344 & 3057.826 & 1514.644 & 1515.797 & 1516.26 \\
\hline 3070.2865 & 3077.057 & 3111.618 & 1516.5379 & 1537.061 & 1537.543 \\
\hline
\end{tabular}




$\begin{array}{rrr}1537.6334 & 1577.257 & 1616.656 \\ 1628.2528 & 1638.387 & 1660.935 \\ 1688.9573 & 1689.51 & 3013.22 \\ 3013.3593 & 3015.046 & 3015.17 \\ 3018.1203 & 3018.454 & 3057.277 \\ 3057.3346 & 3057.488 & 3069.649 \\ 3069.8233 & 3070.483 & 3076.699 \\ 3076.8371 & 3077.008 & 3111.105 \\ 3111.1329 & 3111.223 & 3160.542 \\ 3160.5804 & 3171.019 & 3173.644 \\ 3173.8784 & 3180.545 & 3184.214 \\ 3188.9858 & 3251.506 & 3251.515\end{array}$

B $(\mathrm{DP}=5)$

\begin{tabular}{rrr}
6.9463 & 10.0508 & 14.5669 \\
18.1715 & 20.466 & 33.3287 \\
45.9527 & 58.5578 & 59.4202 \\
66.8401 & 71.871 & 87.8876 \\
94.6594 & 122.8846 & 124.5337 \\
130.021 & 135.4477 & 139.0572 \\
139.3383 & 147.1334 & 149.2517 \\
167.654 & 170.2199 & 173.8744 \\
174.33 & 191.283 & 198.2467 \\
201.061 & 203.715 & 215.9856 \\
217.8629 & 227.9176 & 228.2037 \\
228.8516 & 234.9562 & 261.7251 \\
310.4777 & 336.4232 & 336.7229 \\
366.8574 & 370.5009 & 393.0925 \\
426.2181 & 429.755 & 478.8153 \\
505.9474 & 516.1446 & 518.5197 \\
522.1233 & 541.61 & 545.5934 \\
560.0531 & 586.7393 & 592.3016 \\
595.2465 & 604.4003 & 617.9171 \\
621.1078 & 630.4369 & 636.7816 \\
645.4594 & 653.3956 & 677.5561 \\
706.0891 & 716.9242 & 724.2856 \\
748.8726 & 778.586 & 795.4549 \\
803.3228 & 805.251 & 864.235 \\
867.0872 & 867.8322 & 868.914 \\
872.5211 & 875.1809 & 877.288 \\
882.4662 & 887.0462 & 907.0674 \\
& & \\
\hline
\end{tabular}

\begin{tabular}{|c|c|c|}
\hline 908.4593 & 910.9569 & 911.9273 \\
\hline 912.311 & 915.4807 & 916.9465 \\
\hline 939.4114 & 939.6362 & 942.1585 \\
\hline 942.3429 & 943.3197 & 968.0301 \\
\hline 969.8988 & 975.0507 & 977.576 \\
\hline 982.442 & 985.5918 & 986.9282 \\
\hline 994.968 & 1000.9753 & 1020.267 \\
\hline 1020.6876 & 1027.2482 & 1027.3355 \\
\hline 1041.3086 & 1042.4702 & 1045.7566 \\
\hline 1055.4052 & 1055.5362 & 1067.1745 \\
\hline 1068.5415 & 1070.2266 & 1071.8207 \\
\hline 1072.668 & 1163.0968 & 1163.7962 \\
\hline 1164.4105 & 1164.9116 & 1165.3822 \\
\hline 1220.8489 & 1222.5159 & 1225.3311 \\
\hline 1226.7067 & 1230.1281 & 1233.9597 \\
\hline 1240.4691 & 1244.8685 & 1247.58 \\
\hline 1248.1869 & 1253.7385 & 1255.1641 \\
\hline 1260.4096 & 1264.3806 & 1266.5991 \\
\hline 1299.8543 & 1300.0417 & 1302.7644 \\
\hline 1303.4718 & 1304.8619 & 1308.5788 \\
\hline 1314.1724 & 1326.939 & 1328.9584 \\
\hline 1333.5239 & 1339.0774 & 1340.6974 \\
\hline 1341.5513 & 1343.8238 & 1344.5921 \\
\hline 1355 & 13 & 555 \\
\hline 1357.054 & 5429 & 1359.1481 \\
\hline 1364.3553 & 1365.2209 & 1366.7604 \\
\hline 1374.407 & 1409.5949 & 1409.7448 \\
\hline 1417.4211 & 1418.5894 & 1425.6753 \\
\hline 1474.7572 & 1475.4482 & 1511.2553 \\
\hline 1513.2392 & 1513.8411 & 1514.2599 \\
\hline 1514.5726 & 1515.2141 & 1515.4682 \\
\hline 1515.5376 & 1515.6702 & 1516.2031 \\
\hline 1535.5033 & 1536.7278 & 1536.8835 \\
\hline 1537.1013 & 1537.4788 & 1553.9918 \\
\hline 1583.3872 & 1605.4174 & 1609.4302 \\
\hline 1627.3255 & 1633.1391 & 1636.0778 \\
\hline 1653.2319 & 1663.4366 & 1688.7295 \\
\hline 1688.8191 & 3012.9048 & 3013.0502 \\
\hline 3014.1882 & 3014.3225 & 3014.7669 \\
\hline 3014.8135 & 3015.198 & 3015.4924 \\
\hline 3018.4151 & 3018.4216 & 3057.2222 \\
\hline 3057.3003 & 3057.3427 & 3057.4021 \\
\hline
\end{tabular}




\begin{tabular}{|c|c|c|}
\hline 3057.4455 & 3069.6115 & 3069.7169 \\
\hline 3070.5153 & 3070.6948 & 3070.7253 \\
\hline 3076.6169 & 3076.6908 & 3076.709 \\
\hline 3076.9716 & 3076.9862 & 3110.6786 \\
\hline 3110.7982 & 3110.9048 & 3111.052 \\
\hline 3111.1327 & 3160.4385 & 3160.9233 \\
\hline 3169.3879 & 3171.4149 & 3173.4824 \\
\hline 3173.8808 & 3174.3555 & 3181.2 \\
\hline 3182.7454 & 3185.758 & 3188.681 \\
\hline 3190.3696 & 3251.4771 & 3251.7933 \\
\hline $\mathbf{B}(\mathrm{DP}=10)$ & & \\
\hline-5.4134 & 2.0322 & 2.2362 \\
\hline 5.0137 & 7.5194 & 10.0105 \\
\hline 12.6034 & 14.9353 & 16.1646 \\
\hline 19.2105 & 23.6904 & 24.2736 \\
\hline 25.2137 & 31.8053 & 33.36 \\
\hline 43.4632 & 47.2093 & 64.423 \\
\hline 65.8409 & 68.9795 & 72.4951 \\
\hline 75.9012 & 76.3217 & 78.175 \\
\hline 79.9922 & 87.144 & 88.7828 \\
\hline 92.498 & 111.0417 & 124.0798 \\
\hline 125.5961 & 135.8156 & 143.879 \\
\hline 154.6846 & 155.1461 & 158.1855 \\
\hline 161.6648 & 162.0517 & 162.9983 \\
\hline 164.9589 & 167.2389 & 167.2879 \\
\hline 184.5236 & 197.4927 & 199.2695 \\
\hline 202.5284 & 202.53 & 202.6745 \\
\hline 202.7182 & 205.0186 & 205.6721 \\
\hline 205.9659 & 207.2678 & 213.5943 \\
\hline 217.7165 & 221.4326 & 228.0282 \\
\hline 233.7759 & 236.9666 & 246.3596 \\
\hline 254.8117 & 261.4404 & 264.4731 \\
\hline 266.1525 & 298.0518 & 326.6137 \\
\hline 326.6144 & 330.5899 & 357.6196 \\
\hline 359.1769 & 359.2588 & 363.6509 \\
\hline 371.189 & 380.5874 & 382.091 \\
\hline 392.0281 & 396.5086 & 413.0638 \\
\hline 429.0169 & 490.0567 & 493.4815 \\
\hline 515.3692 & 529.6113 & 531.151 \\
\hline 535.005 & 536.7594 & 540.0474 \\
\hline
\end{tabular}

$\begin{array}{rrr}546.8916 & 551.6708 & 554.3066 \\ 559.0942 & 561.6109 & 572.3027 \\ 577.8728 & 578.324 & 588.313 \\ 601.7198 & 611.8107 & 617.7083 \\ 648.0459 & 648.0511 & 688.4965 \\ 688.7393 & 692.7335 & 693.6721 \\ 696.2283 & 708.1816 & 708.1828 \\ 709.4688 & 728.1982 & 748.8668 \\ 777.5277 & 786.5 & 803.8012 \\ 804.2217 & 805.8073 & 807.9427 \\ 810.2627 & 812.5577 & 814.441 \\ 815.8378 & 816.1998 & 816.4957 \\ 822.5717 & 822.5745 & 825.481 \\ 832.0989 & 836.6449 & 838.9026 \\ 839.2988 & 839.9479 & 880.7035 \\ 881.3221 & 882.3126 & 883.5928 \\ 885.2179 & 886.5601 & 887.9428 \\ 889.0652 & 889.8206 & 915.8953 \\ 915.8986 & 930.8168 & 930.8186 \\ 936.2755 & 936.3376 & 936.675 \\ 936.8316 & 937.1869 & 937.4719 \\ 937.5186 & 937.5616 & 979.031 \\ 980.0362 & 981.1756 & 981.2874 \\ 982.2181 & 982.8941 & 983.8001 \\ 984.9887 & 985.2857 & 985.6964 \\ 988.1616 & 988.1767 & 988.3007 \\ 991.8563 & 995.3816 & 998.1551 \\ 1000.0217 & 1000.9574 & 1001.2199 \\ 1022.8773 & 1022.8795 & 1041.6542 \\ 1041.6737 & 1055.174 & 1055.6182 \\ 1056.1755 & 1056.797 & 1057.4576 \\ 1058.0653 & 1058.5712 & 1058.8463 \\ 1060.5422 & 1060.5427 & 1098.2384 \\ 1098.5262 & 1099.0297 & 1099.6873 \\ 1100.4551 & 1101.1772 & 1101.8875 \\ 1102.4729 & 1102.8907 & 1103.1274 \\ 1185.7538 & 1185.7552 & 1187.9701 \\ 1187.9886 & 1188.3675 & 1188.612 \\ 1188.8988 & 1189.1632 & 1189.3922 \\ 1225.9344 & 1226.115 & 1226.602\end{array}$




\begin{tabular}{|c|c|c|}
\hline 1227.7009 & 1227.7093 & 1235.5216 \\
\hline 1235.5321 & 1238.6708 & 1238.6794 \\
\hline 1239.2444 & 1239.2494 & 1239.384 \\
\hline 1239.5957 & 1239.6572 & 1239.7688 \\
\hline 1245.7178 & 1249.7351 & 1257.4586 \\
\hline 1267.4079 & 1268.868 & 1281.3115 \\
\hline 1283.0785 & 1289.4017 & 1290.9734 \\
\hline 1292.1707 & 1309.926 & 1309.9631 \\
\hline 1310.5099 & 1311.5218 & 1313.0238 \\
\hline 1313.126 & 1313.4134 & 1313.4773 \\
\hline 1314.8789 & 1315.43 & 1315.9412 \\
\hline 1316.204 & 1316.4712 & 1319.0457 \\
\hline 1319.6576 & 1326.0907 & 1330.4109 \\
\hline 1331.1769 & 1335.0057 & 1339.2207 \\
\hline 1388.7692 & 1388.7808 & 1401.3276 \\
\hline 1401.5347 & 1402.341 & 1402.8557 \\
\hline 1404.6454 & 1410.9963 & 1421.7915 \\
\hline 1435.6522 & 1467.3774 & 1468.4375 \\
\hline 1485.8736 & 1496.8844 & 1496.9407 \\
\hline 1496.9931 & 1497.0218 & 1497.0697 \\
\hline 1497.1092 & 1497.2664 & 1497.2711 \\
\hline 1497.9826 & 1497.9839 & 1502.7425 \\
\hline 1509.2473 & 1511.7278 & 1512.8332 \\
\hline 1513.3912 & 1513.6835 & 1513.7917 \\
\hline 1514.6063 & 1514.6087 & 1529.8353 \\
\hline 1549.1501 & 1564.1211 & 1574.4143 \\
\hline 1578.3345 & 1595.7855 & 1597.2744 \\
\hline 1613.4293 & 1618.7145 & 1628.4459 \\
\hline 1638.4078 & 1641.83 & 1651.657 \\
\hline 1655.2546 & 1660.1611 & 1665.5291 \\
\hline 1668.9141 & 1670.9955 & 1674.3387 \\
\hline 1693.5297 & 1693.5303 & 3044.9086 \\
\hline 3044.9311 & 3045.6113 & 3045.6149 \\
\hline 3045.6958 & 3045.7241 & 3045.8192 \\
\hline 3045.8272 & 3045.9134 & 3045.9179 \\
\hline 3050.2291 & 3050.2321 & 3050.3233 \\
\hline 3050.338 & 3050.4652 & 3050.5043 \\
\hline 3050.654 & 3051.0293 & 3051.6943 \\
\hline 3051.7652 & 3081.2853 & 3081.2909 \\
\hline 3081.6125 & 3081.6174 & 3081.6413 \\
\hline 3081.6424 & 3081.6593 & 3081.6602 \\
\hline 3081.6787 & 3081.6811 & 3096.5454 \\
\hline
\end{tabular}

$\begin{array}{rrr}3096.5474 & 3096.5689 & 3096.577 \\ 3096.6323 & 3096.7123 & 3096.8178 \\ 3096.9388 & 3097.5224 & 3097.5294 \\ 3148.7019 & 3148.852 & 3149.0636 \\ 3149.3604 & 3149.7576 & 3150.381 \\ 3150.9263 & 3151.6335 & 3152.4225 \\ 3153.294 & 3153.7981 & 3155.9263 \\ 3156.809 & 3157.6511 & 3158.4587 \\ 3159.0325 & 3159.7432 & 3160.2563 \\ 3160.6347 & 3160.8585 & 3169.1664 \\ 3169.1721 & 3251.5509 & 3251.5568\end{array}$

B $(\mathrm{DP}=15)$

\begin{tabular}{|c|c|c|}
\hline-0.4414 & 1.5155 & 2.9247 \\
\hline 4.6754 & 5.3443 & 6.2738 \\
\hline 7.3495 & 7.9965 & 10.2498 \\
\hline 10.4683 & 11.4534 & 13.0489 \\
\hline 13.2578 & 14.85 & 15.1787 \\
\hline 16.5779 & 21.358 & 21.489 \\
\hline 22.4124 & 23.7724 & 29.9676 \\
\hline 36.8413 & 40.2442 & 44.2033 \\
\hline 48.4669 & 52.1586 & 52.931 \\
\hline 57.2523 & 58.1416 & 59.2772 \\
\hline 60.6526 & 61.3813 & 63.9271 \\
\hline 64.2652 & 66.3388 & 68.0764 \\
\hline 68.37 & 69.8409 & 72.4568 \\
\hline 76.6739 & 78.9841 & 90.4647 \\
\hline 90.6939 & 113.6362 & 123.7204 \\
\hline 24.3204 & 126.8226 & 127.2352 \\
\hline 28.2471 & 129.5693 & 130.2061 \\
\hline 132.462 & 133.9713 & 135.3735 \\
\hline 6.1451 & 137.011 & 137.368 \\
\hline 37.7644 & 137.8721 & 138.7991 \\
\hline 9.9379 & 145.723 & 148.617 \\
\hline 19.9909 & 166.1677 & 167.5932 \\
\hline 58.3287 & 169.115 & 169.8267 \\
\hline 70.2163 & 171.0163 & 171.893 \\
\hline 72.6559 & 173.5643 & 173.8325 \\
\hline 74.7544 & 175.5381 & 176.056 \\
\hline 88.8439 & 190.2306 & 192.5123 \\
\hline 95.2799 & 195.5317 & 198.6579 \\
\hline
\end{tabular}




\begin{tabular}{|c|c|c|}
\hline 201.2439 & 203.0705 & 204.6576 \\
\hline 208.2619 & 212.1706 & 216.0656 \\
\hline 217.2014 & 224.9723 & 225.1135 \\
\hline 25.9518 & 227.716 & 228.2987 \\
\hline 229.2201 & 229.8302 & 230.5208 \\
\hline 230.9504 & 231.6013 & 233.2112 \\
\hline 234.326 & 235.0792 & 236.1526 \\
\hline 237.4948 & 238.4939 & 238.7957 \\
\hline 239.4452 & 243.1219 & 261.888 \\
\hline 283.3218 & 306.1836 & 329.8106 \\
\hline 36.4136 & 336.5996 & 353.5163 \\
\hline 357.2491 & 360.4672 & 363.304 \\
\hline 367.2975 & 372.8408 & 376.9911 \\
\hline 379.9225 & 388.5211 & 397.8234 \\
\hline 399.3322 & 408.1337 & 418.6773 \\
\hline 420.7427 & 429.0447 & 438.3989 \\
\hline 441.3564 & 444.9504 & 458.9146 \\
\hline 476.2459 & 491.6268 & 504.3367 \\
\hline 510.2704 & 510.815 & 514.1725 \\
\hline 515.0408 & 518.1028 & 520.9986 \\
\hline 523.3733 & 528.0246 & 529.5624 \\
\hline 530.735 & 534.31 & 534.8165 \\
\hline 537.2661 & 543.8683 & 544.8747 \\
\hline 548.4892 & 557.5179 & 567.123 \\
\hline 575.885 & 585.1685 & 591.8546 \\
\hline 592.5395 & 593.2965 & 597.1878 \\
\hline 603.9494 & 606.8199 & 612.9787 \\
\hline 613.3239 & 614.5781 & 615.2931 \\
\hline 616.3007 & 620.0548 & 620.4633 \\
\hline 620.7317 & 624.5757 & 625.7418 \\
\hline 628.5642 & 631.4986 & 634.4885 \\
\hline 637.6525 & 638.5714 & 640.7564 \\
\hline 641.9139 & 644.0614 & 645.1645 \\
\hline 650.8947 & 653.0475 & 655.0862 \\
\hline 661.201 & 670.6884 & 680.6622 \\
\hline 691.5239 & 703.6358 & 713.3857 \\
\hline 718.6446 & 723.1545 & 732.6198 \\
\hline 742.87 & 751.702 & 765.6 \\
\hline 774.5276 & 781.5972 & 787.900 \\
\hline 792.7566 & 796.8713 & 799.9091 \\
\hline 801.9617 & 803.4504 & 804.023 \\
\hline 804.4401 & 805.7529 & 805.9 \\
\hline
\end{tabular}

\begin{tabular}{|c|c|c|}
\hline 806 & 86 & 6 \\
\hline 866.1882 & 867.0555 & 867.2729 \\
\hline 867.5189 & 867.6128 & 67.9064 \\
\hline 868.1352 & 868.6455 & 868.7084 \\
\hline 869.8626 & 869.9447 & 7705545 \\
\hline 872.3612 & 873.1192 & 873.9888 \\
\hline 876.5183 & 876.7675 & 878. \\
\hline 879.8481 & 880.6461 & 881. \\
\hline 882.675 & 883.7073 & 884.8055 \\
\hline 885.6155 & 886.9749 & \\
\hline 905.6865 & 906.0924 & 908.1405 \\
\hline 908.5636 & 908.8787 & 910.3595 \\
\hline 910.9116 & 911.4581 & 911 \\
\hline 912.256 & 912.6511 & 912.5 \\
\hline 915.1222 & 915.7401 & 916. \\
\hline 916.2756 & 916.5563 & 940. \\
\hline 940.3074 & 942.3102 & 942.5013 \\
\hline 942.5708 & 942.6299 & 942.8276 \\
\hline 942.9886 & 943.0551 & 943.201 \\
\hline 943.4586 & 943.736 & 944.1 \\
\hline 944.1851 & 944.3996 & 963.7164 \\
\hline 965.936 & 966.8277 & 968.5683 \\
\hline 969.3297 & 970.5408 & 970. \\
\hline 972.5347 & 974.0613 & 974.7642 \\
\hline 976.7249 & 977.5977 & 979.0473 \\
\hline 980.4002 & 981.7112 & 982.6296 \\
\hline 983.7921 & 984.7975 & 985.6514 \\
\hline 986.9356 & 987.1652 & 989.1551 \\
\hline 992.3859 & 995.4051 & \\
\hline 1000.0405 & 1001.525 & 18 \\
\hline 1003.0949 & 1020.191 & 1021.059 \\
\hline 1027.4722 & 1027.873 & 1040.914 \\
\hline 1041.1366 & 1041.285 & 1041.501 \\
\hline 1041.696 & 1041.93 & 1042.421 \\
\hline 1043.0876 & 1044.035 & 1045.184 \\
\hline 1046.3768 & 1047.198 & 1047.928 \\
\hline 1056.0494 & 1056.501 & 1066.089 \\
\hline 1066 & 106 & 10 \\
\hline 1067.7335 & 1068.217 & 1068.79 \\
\hline 1069.4136 & 1070.045 & 1070.6 \\
\hline 1071.2042 & 1071.619 & 1072.01 \\
\hline 1072.2684 & 1072.788 & 1163 \\
\hline
\end{tabular}




\begin{tabular}{|c|c|c|}
\hline & & \\
\hline & 1164.368 & 1164.634 \\
\hline & 7 & \\
\hline & & \\
\hline & 2 & 12 \\
\hline 16 & 1 & 880 \\
\hline 2 & 77 & 64 \\
\hline 1223.521 & 502 & 225.369 \\
\hline 1 & & 05 \\
\hline & & \\
\hline & 4 & 08 \\
\hline & & 15 \\
\hline 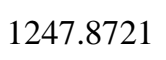 & 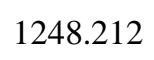 & ? \\
\hline 019 & 387 & 42 \\
\hline 2 & 6 & 29 \\
\hline & & \\
\hline .0487 & 1 & \\
\hline 078 & 88 & 1264.087 \\
\hline 19 & 18 & \\
\hline o & 4 & 26 \\
\hline 00 & & \\
\hline & & \\
\hline & & \\
\hline & 76 & 305.503 \\
\hline 801 & 200 & 53 \\
\hline 454 & 6 & 21 \\
\hline 34 & & \\
\hline & & \\
\hline & & \\
\hline & & \\
\hline 41 & .04 & 382 \\
\hline 10 & 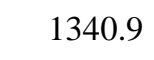 & 59 \\
\hline 1342 & 342 & 104 \\
\hline 1545.0552 & 104 & 1 \\
\hline 1345.5382 & 1345.818 & 1355.115 \\
\hline & & \\
\hline $1356 t r$ & 6 & 26 \\
\hline 10 & 1357.306 & 79 \\
\hline 13 & $10 J$ & 8 \\
\hline 1358.3040 & 1538.004 & 1358.747 \\
\hline 175 & 1359.087 & 1359.769 \\
\hline & 1359.958 & 150 \\
\hline
\end{tabular}

\begin{tabular}{|c|c|c|}
\hline 1365.1101 & 1365.865 & 1500.5 \\
\hline 1368.6639 & 1372.077 & 1374.661 \\
\hline 376.3063 & 1376.823 & 1410.079 \\
\hline 410.3707 & 1418.018 & 1418108 \\
\hline 418.4115 & 1418.56 & 1418.755 \\
\hline 1419.0195 & 1419.483 & 1420.456 \\
\hline 1422.0079 & 1424.383 & 1427.685 \\
\hline 1431.3107 & 1434.366 & 1474.14 \\
\hline 1475.4397 & 1505.739 & 1508. \\
\hline 1510.1362 & 1511.34 & 1512.038 \\
\hline 1512.4947 & 1512.745 & 1512.97 \\
\hline 1513.23 & 1513.403 & S \\
\hline 1513.6711 & 1513.786 & 1015.03 \\
\hline 1514.0451 & 1514.18 & 1514.36 \\
\hline 1514.3876 & 1514.422 & 1514.48 \\
\hline 1514.715 & 1514.825 & 1514.833 \\
\hline 1515.0881 & 1515.117 & 1515.20 \\
\hline 1515.4012 & 1515.545 & 15 \\
\hline 1516.5283 & 1527.886 & 1531.5 \\
\hline 1534.465 & 1535.52 & 1535.81 \\
\hline 1535.9554 & 1536.129 & 1536.35 \\
\hline 1536.5823 & 1536.588 & 1536.735 \\
\hline 1536.9935 & 1537.289 & 1537.588 \\
\hline 1537.9266 & 1539.924 & 1542.627 \\
\hline 1548.8428 & 1558.352 & 1568.9 \\
\hline 1579.4773 & 1588.155 & 158 \\
\hline 1593.7326 & 1598.692 & 1600. \\
\hline 1607.1824 & 1609.296 & 1614.713 \\
\hline 1618.0438 & 1621.24 & 1626.048 \\
\hline 1627.3774 & 1630.656 & 1633.845 \\
\hline 1633.9689 & 1635.745 & 1642 \\
\hline 1649.0559 & 1654.242 & 1658.30 \\
\hline 1661.161 & 1663.288 & 1663.697 \\
\hline 1688.1515 & 1688.875 & 3012 \\
\hline 3013.5761 & 3013.785 & 3013 \\
\hline 3013.9325 & 3013.984 & 3014.0 \\
\hline 3014.1784 & 3014.196 & 3014.265 \\
\hline 3014.3026 & 3014.419 & 3014.48 \\
\hline 3014.5405 & 3014.551 & 3014.57 \\
\hline 3014.6487 & 3014.697 & 3014. \\
\hline 3014.8754 & 3015.042 & 3015. \\
\hline 45 & 3015.23 & 30 \\
\hline
\end{tabular}




\begin{tabular}{|c|c|c|c|c|c|}
\hline 3015.3612 & 3015.402 & 3015.863 & 873.528 & 894.2746 & 917.2297 \\
\hline 3017.7968 & 3018.917 & 3057.216 & 919.4837 & 934.0569 & 964.0026 \\
\hline 3057.2659 & 3057.282 & 3057.286 & 1004.6451 & 1019.5078 & 1028.6729 \\
\hline 3057.29 & 3057.302 & 3057.32 & 1064.6791 & 1092.7011 & 1105.2527 \\
\hline 3057.3243 & 3057.339 & 3057.346 & 1114.9161 & 1171.6729 & 1197.7439 \\
\hline 3057.3733 & 3057.402 & 3057.413 & 1219.352 & 1284.9644 & 1291.8808 \\
\hline 3057.4338 & 3057.494 & 3069.37 & 1319.7725 & 1335.8465 & 1391.2038 \\
\hline 3069.8184 & 3070.229 & 3070.331 & 1391.2742 & 1400.9128 & 1410.3747 \\
\hline 3070.6153 & 3070.648 & 3070.709 & 1418.6927 & 1469.8407 & 1478.5982 \\
\hline 3070.736 & 3070.807 & 3070.851 & 1504.346 & 1513.4408 & 1522.1314 \\
\hline 3070.8737 & 3070.939 & 3070.997 & 1534.6223 & 1630.3784 & 1689.7812 \\
\hline 3071.0584 & 3071.079 & 3076.508 & 1692.0879 & 3010.5551 & 3010.9537 \\
\hline 3076.5641 & 3076.575 & 3076.648 & 3030.8577 & 3036.0891 & 3062.9513 \\
\hline 3076.6655 & 3076.741 & 3076.751 & 3063.5483 & 3079.1139 & 3083.218 \\
\hline 3076.7901 & 3076.826 & 3076.848 & 3173.7627 & 3176.1191 & 3189.5554 \\
\hline 3076.9321 & 3077.004 & 3077.036 & 3212.5371 & 3253.0875 & 3254.2995 \\
\hline 3077.0849 & 3077.159 & 3110.422 & & & \\
\hline 3110.4357 & 3110.548 & 3110.587 & $\mathrm{C}(\mathrm{DP}=3)$ & & \\
\hline 3110.5938 & 3110.676 & 3110.685 & 16.8935 & 17.6566 & 29.8666 \\
\hline 3110.699 & 3110.827 & 3110.88 & 46.8887 & 57.8455 & 76.5579 \\
\hline 3110.908 & 3110.954 & 3110.987 & 81.5238 & 98.7188 & 106.9362 \\
\hline 3111.3037 & 3111.311 & 3162.451 & 111.8028 & 118.3987 & 119.746 \\
\hline 3162.7062 & 3169.792 & 3170.353 & 132.8332 & 155.0556 & 168.207 \\
\hline 3171.1027 & 3171.595 & 3172.595 & 170.8541 & 188.6885 & 190.9155 \\
\hline 3173.2144 & 3173.546 & 3174.374 & 232.2136 & 246.2003 & 284.2112 \\
\hline 3174.8916 & 3175.129 & 3175.334 & 308.9146 & 311.7863 & 313.8862 \\
\hline 3175.8757 & 3176.377 & 3177.54 & 328.2298 & 350.6605 & 361.6938 \\
\hline 3177.585 & 3182.841 & 3183.78 & 409.2103 & 419.531 & 421.6556 \\
\hline 3184.2572 & 3184.8 & 3185.625 & 464.2412 & 477.9547 & 483.6667 \\
\hline 3186.6968 & 3187.438 & 3188.255 & 489.9676 & 496.2046 & 527.5555 \\
\hline 3189.4105 & 3190.459 & 3190.906 & 528.6572 & 535.0831 & 546.5041 \\
\hline 3191.3315 & 3191.674 & 3192.917 & 549.3558 & 606.7506 & 617.7047 \\
\hline 3193.114 & 3252.552 & 3252.718 & 623.5499 & 658.4265 & 695.8272 \\
\hline & & & 710.7034 & 719.5298 & 762.7081 \\
\hline$(\mathrm{DP}=1)$ & & & 785.033 & 838.0927 & 840.1441 \\
\hline 90.0612 & 98.337 & 108.787 & 857.9817 & 867.2794 & 875.434 \\
\hline 118.4895 & 233.3177 & 267.7862 & 880.1437 & 888.1976 & 896.2545 \\
\hline 306.5525 & 325.7956 & 412.4362 & 902.7498 & 909.3411 & 909.5155 \\
\hline 423.3795 & 458.3008 & 509.1598 & 913.4605 & 913.6679 & 932.9438 \\
\hline 550.9565 & 586.5005 & 676.882 & 935.7113 & 937.8274 & 969.9977 \\
\hline 684.3565 & 743.16 & 858.9693 & 972.9118 & 978.4271 & 986.697 \\
\hline
\end{tabular}




\begin{tabular}{rrr}
997.3204 & 1018.1656 & 1019.3156 \\
1045.8614 & 1046.5103 & 1081.7847 \\
1082.863 & 1091.4365 & 1099.1539 \\
1105.1968 & 1107.4432 & 1112.2921 \\
1113.5639 & 1113.7433 & 1171.8181 \\
1172.1562 & 1173.0698 & 1179.9078 \\
1199.8668 & 1205.3926 & 1216.699 \\
1220.7792 & 1225.0973 & 1280.3167 \\
1285.0134 & 1293.1409 & 1293.4348 \\
1294.861 & 1298.101 & 1322.4698 \\
1322.6418 & 1325.7343 & 1333.7829 \\
1337.4195 & 1349.4707 & 1370.698 \\
1390.0184 & 1391.4171 & 1391.7759 \\
1391.8023 & 1392.1974 & 1396.3175 \\
1401.7465 & 1408.1569 & 1411.9468 \\
1413.1418 & 1415.7757 & 1419.6038 \\
1426.844 & 1428.6191 & 1474.7398 \\
1475.1808 & 1503.1743 & 1503.7848 \\
1504.3065 & 1512.0416 & 1512.9057 \\
1512.9409 & 1521.691 & 1521.8239 \\
1521.8336 & 1533.619 & 1533.6506 \\
1533.7498 & 1584.9466 & 1613.1941 \\
1625.2441 & 1635.1784 & 1655.6603 \\
1685.5611 & 1685.7806 & 3007.997 \\
3008.1059 & 3008.6443 & 3009.0662 \\
3009.6563 & 3009.9973 & 3030.4358 \\
3030.6891 & 3030.8246 & 3035.402 \\
3035.6091 & 3035.6286 & 3058.2798 \\
3058.4702 & 3060.2106 & 3060.3088 \\
3062.2062 & 3062.3077 & 3078.6036 \\
3078.85 & 3079.3779 & 3082.9596 \\
3083.0145 & 3083.2384 & 3175.7702 \\
3175.8157 & 3194.9757 & 3195.0019 \\
3248.1638 & 3254.8381 & 3254.9714 \\
& & \\
\hline
\end{tabular}

\begin{tabular}{|c|c|c|}
\hline 84.5342 & 92.1274 & 97.8896 \\
\hline 102.0534 & 109.1859 & 112.2707 \\
\hline 115.492 & 119.4214 & 119.7622 \\
\hline 154.1922 & 156.5467 & 156.8707 \\
\hline 166.3646 & 166.6126 & 176.625 \\
\hline 181.9612 & 189.1569 & 189.2855 \\
\hline 193.0234 & 203.6234 & 228.741 \\
\hline 245.6095 & 252.7344 & 281.4067 \\
\hline 307.3694 & 310.6211 & 311.7039 \\
\hline 312.8072 & 315.4553 & 319.9626 \\
\hline 351.2591 & 355.759 & $361.255 \mathrm{c}$ \\
\hline 384.726 & 405.8809 & 417.6806 \\
\hline 420.4493 & 426.1018 & 463.4345 \\
\hline 466.177 & 475.2775 & 482.4803 \\
\hline 486.2228 & 489.3347 & 493.9902 \\
\hline 494.8549 & 498.7303 & 525.0228 \\
\hline 527.8189 & 530.6279 & 537.5572 \\
\hline 538.603 & 541.7091 & 545.5494 \\
\hline 575.836 & 606.2279 & 618.1414 \\
\hline 619.8126 & 631.1345 & 631.462 \\
\hline 651.7692 & 679.5212 & 699.9993 \\
\hline 712.6662 & 717.7053 & 750.9388 \\
\hline 769.8465 & 782.5726 & 789.3841 \\
\hline 837.6852 & 838.093 & 839.6512 \\
\hline 842.9633 & 857.5815 & 862.4905 \\
\hline 870.4933 & 872.9899 & $878.960 \mathrm{c}$ \\
\hline 881.3981 & 887.5282 & 889.9921 \\
\hline 895.3865 & 901.0586 & 905.2657 \\
\hline 908.5781 & 909.1259 & 911.5811 \\
\hline 912.6066 & 913.115 & 913.1281 \\
\hline 932.0151 & 933.7681 & 935.5024 \\
\hline 937.1962 & 938.159 & 966.2858 \\
\hline 971.5842 & 974.6277 & $976.615 \mathrm{c}$ \\
\hline 984.4192 & 985.713 & 995.8174 \\
\hline 997.1481 & 1002.601 & 1018.826 \\
\hline 1018.8852 & 1046.418 & 1046.434 \\
\hline 1078.7643 & 1080.614 & 1082.642 \\
\hline 1085.3966 & 1094.713 & 1097.604 \\
\hline 1099.3612 & 1102.128 & 1106.879 \\
\hline 1106.9002 & 1111.779 & 1112. \\
\hline 1112.3238 & 1113.556 & 1113. \\
\hline 1167.9793 & 1171.634 & 1171.918 \\
\hline
\end{tabular}




\begin{tabular}{|c|c|c|}
\hline & 8 & $17 J$ \\
\hline 873201 & 1199.951 & 1204726 \\
\hline 1 & 3 & $78 \times 1>$ \\
\hline - & 225.063 & Sc \\
\hline 201.0301 & 4 & t \\
\hline 1292.3598 & 293.328 & re \\
\hline 1294.4275 & 1294.7 & 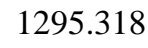 \\
\hline & & \\
\hline 65 & & \\
\hline & 8 & \\
\hline 34 & 1 & $0 .>->$ \\
\hline 77.4486 & 2 & 1391.204 \\
\hline 94 & & 1391.526 \\
\hline & & \\
\hline & 396.688 & \\
\hline 6 & 9 & \\
\hline & & \\
\hline & & 424.16 \\
\hline 32 & 9 & \\
\hline & & \\
\hline & & \\
\hline 1504.1795 & 511.577 & \\
\hline 586 & 8 & 15100 \\
\hline 15216507 & 1521.661 & 1501 \\
\hline 85 & 5 & 1533.388 \\
\hline & & \\
\hline & & \\
\hline 1604 & 468 & \\
\hline 16230 & 1 & \\
\hline 04 & 4 & 1685.475 \\
\hline 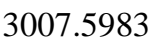 & 3007.723 & - 3001. \\
\hline 3007.9621 & 3008.036 & 3008.35 \\
\hline 88 & & \\
\hline & & \\
\hline 303 & 7 & 13 \\
\hline 30 & 2 & 01 \\
\hline 30 & 8 & 00 \\
\hline 5Uग0.01J & 5050.4 & 05 \\
\hline 3058.9487 & 3059.042 & 5000. \\
\hline 3060.1599 & 3062.038 & 3062 \\
\hline 3078.5212 & 3078.591 & 3078 \& \\
\hline 078.8388 & 3079.139 & 3082 \\
\hline
\end{tabular}

$\begin{array}{lll}3082.8866 & 3082.893 & 3082.962 \\ 3082.9766 & 3175.915 & 3175.924 \\ 3195.3619 & 3195.367 & 3216.958 \\ 3219.0354 & 3221.275 & 3238.258 \\ 3239.5319 & 3243.469 & 3247.417 \\ 3249.7885 & 3255.01 & 3255.025\end{array}$$$
\mathrm{C}(\mathrm{DP}=10)
$$$$
\begin{array}{lll}
2.8821 & 5.2998 & 6.9336 \\
7.0335 & 7.7617 & 9.7201
\end{array}
$$$$
9.8204 \quad 10.5075 \quad 13.6524
$$$$
\begin{array}{lll}
17.0313 \quad 17.2572 \quad 22.1984
\end{array}
$$$$
\begin{array}{lll}
29.5828 & 33.6553 \quad 39.3803
\end{array}
$$$$
46.907 \quad 48.3298 \quad 51.4143
$$$$
52.1984 \quad 52.5976 \quad 54.1963
$$$$
\begin{array}{lll}
54.6196 & 57.4128 & 62.1075
\end{array}
$$$$
\begin{array}{lll}
65.6277 & 69.0444 & 80.6621
\end{array}
$$$$
82.0455 \quad 86.6983 \quad 91.9474
$$$$
95.1039 \quad 97.128 \quad 101.623
$$$$
106.2624 \quad 109.7198 \quad 110.5193
$$$$
\begin{array}{lll}
111.207 \quad 112.2519 \quad 113.3796
\end{array}
$$$$
118.6956 \quad 120.4048 \quad 124.5995
$$$$
148.0615 \quad 154.0126 \quad 155.1921
$$$$
156.5122 \quad 157.0991 \quad 157.7129
$$$$
158.204 \quad 161.9836 \quad 162.7821
$$$$
166.8441 \quad 168.6625 \quad 174.5938
$$$$
\begin{array}{lll}
175.8472 & 176.7109 & 178.1569
\end{array}
$$$$
181.1075 \quad 182.8636 \quad 186.514
$$$$
189.6625 \quad 190.3356 \quad 197.9056
$$$$
202.2106 \quad 202.6579 \quad 208.2132
$$$$
210.1257 \quad 230.7292 \quad 236.4788
$$$$
245.3755 \quad 248.6472 \quad 251.6288
$$$$
269.9596 \quad 287.9572 \quad 305.6698
$$$$
308.7654 \quad 310.2588 \quad 310.7673
$$$$
312.0802 \quad 312.743 \quad 313.0122
$$$$
313.9587 \quad 315.0829 \quad 316.3726
$$$$
317.7369 \quad 337.4485 \quad 351.279
$$$$
\begin{array}{lll}
353.482 & 360.8819 & 365.8405
\end{array}
$$$$
380.9656 \quad 386.0504 \quad 387.8954
$$$$
397.3596 \quad 407.9222 \quad 416.0355
$$$$
418.8278 \quad 420.4539 \quad 423.9404
$$ 


\begin{tabular}{|c|c|c|}
\hline 424.8354 & 462.6373 & 463.9208 \\
\hline 465.96 & 470.1304 & 470.9805 \\
\hline 474.9057 & 478.8555 & 482.1091 \\
\hline 484.3845 & 487.952 & 489.2114 \\
\hline 492.666 & 493.4651 & 493.623 \\
\hline 494.2452 & 494.6288 & 495.9975 \\
\hline 498.4164 & 502.3336 & 522.8376 \\
\hline 523.2913 & 527.1191 & 529.1913 \\
\hline 532.4146 & 533.5802 & 535.6884 \\
\hline 537.3521 & 538.8115 & 541.3043 \\
\hline 544.6382 & 545.2122 & 555.9209 \\
\hline 568.8227 & 583.7374 & 598.5768 \\
\hline 611.6187 & 617.5593 & 619.2601 \\
\hline 624.4496 & 625.0154 & 627.9063 \\
\hline 631.8943 & 632.8162 & 636.8324 \\
\hline 641.3929 & 653.3657 & 667.8497 \\
\hline 682.7898 & 695.7009 & 704.7013 \\
\hline 714.2485 & 716.2963 & 740.9603 \\
\hline 751.7892 & 762.063 & 770.9423 \\
\hline 777.7622 & 782.9125 & 786.7436 \\
\hline 789.3342 & 790.8465 & 836.3203 \\
\hline 836.748 & 837.3127 & 837.9472 \\
\hline 838.261 & 839.39 & 840.9674 \\
\hline 842.7812 & 844.9355 & 856.7408 \\
\hline 858.3457 & 861.6741 & 865.8616 \\
\hline 870.6473 & 873.1353 & 875.2298 \\
\hline 875.8716 & 879.0503 & 880.8479 \\
\hline 882.997 & 885.4778 & 887.6209 \\
\hline 889.0534 & 890.9139 & 892.3637 \\
\hline 896.5401 & 900.2685 & 903.2618 \\
\hline 905.9205 & 908.1967 & 908.7624 \\
\hline 909.1426 & 909.5451 & 910.5926 \\
\hline 910.9814 & 912.1644 & 912.8363 \\
\hline 913.1456 & 913.7905 & 914.0606 \\
\hline 914.3497 & 931.2744 & 931.8319 \\
\hline 932.794 & 933.7485 & 934.5884 \\
\hline 935.4388 & 936.5156 & 937.3083 \\
\hline 937.662 & 938.2299 & 961.351 \\
\hline 965.0754 & 969.0911 & 971.6755 \\
\hline 975.1325 & 976.5618 & 978.9819 \\
\hline 982.6038 & 983.2327 & 986.0121 \\
\hline 986.6736 & 994.8658 & 995.9536 \\
\hline
\end{tabular}

\begin{tabular}{|c|c|c|}
\hline 996.4828 & 998.0293 & 999. \\
\hline 1001.9452 & 1003.974 & 1005.227 \\
\hline 019.5923 & 1019.95 & 1047.609 \\
\hline 047.6438 & 1078.014 & 1078238 \\
\hline 078.9019 & 1080.069 & 108 \\
\hline 1082.642 & 1084.523 & 1087.597 \\
\hline 1091.6815 & 1096.553 & 1097.25 \\
\hline 1098.2241 & 1099.22 & 1101.013 \\
\hline 1102.6709 & 1103.191 & 1104 \\
\hline 1104.4772 & 1107.503 & 1107 \\
\hline 1111.6259 & 1111.964 & 1112.272 \\
\hline 1112.3262 & 1112.382 & 1112.496 \\
\hline 1112.753 & 1113.218 & 1113.504 \\
\hline 1114.3395 & 1157.667 & 11 \\
\hline 1171.2913 & 1171.405 & 1171.59 \\
\hline 1171.8076 & 1171.976 & 1172.173 \\
\hline 1172.3026 & 1172.713 & 1172.905 \\
\hline 1173.4041 & 1179.741 & 1189.573 \\
\hline 1197.1404 & 1201.652 & 1203.956 \\
\hline 1204.7618 & 1205.057 & 1205.442 \\
\hline 1215.2537 & 1215.844 & 1216.621 \\
\hline 1218.1352 & 1220.431 & 122 \\
\hline 1225.1026 & 1225.429 & 1225.965 \\
\hline 1226.3802 & 1281.231 & 1282.267 \\
\hline 1282.7987 & 1283.91 & 1285.68 \\
\hline 1288.5332 & 1291.5 & 129 \\
\hline 1292.5137 & 1292.895 & 1292.996 \\
\hline 1293 & 1293.605 & 1293.973 \\
\hline 1294.0913 & 1294.208 & 1294.518 \\
\hline 1296.8049 & 1299.669 & 1301.448 \\
\hline 1321.5556 & 1322.001 & 1324.009 \\
\hline 1324.6979 & 1324.89 & 1325.1 \\
\hline 1325.5669 & 1326.084 & 1326.601 \\
\hline 1327.1028 & 1331.854 & 1332.711 \\
\hline 1334.0019 & 1336.233 & 1338.105 \\
\hline 1338.9268 & 1343.774 & 1350.696 \\
\hline 1356.9095 & 1358.187 & 136 \\
\hline 1364.7623 & 1372.07 & 1378.7 \\
\hline 1384.5926 & 1388.659 & 1390.2 \\
\hline 1390.4083 & 1390.763 & 1390.9 \\
\hline 1391.0017 & 1391.112 & 1391.24 \\
\hline 391.3489 & 1391.366 & 1391 \\
\hline
\end{tabular}




$\begin{array}{rrr}1391.943 & 1392.182 & 1393.619 \\ 1394.9746 & 1395.526 & 1395.737 \\ 1396.0437 & 1396.724 & 1398.411 \\ 1401.1144 & 1404.677 & 1407.249 \\ 1408.1109 & 1408.817 & 1411.736 \\ 1411.9464 & 1412.149 & 1412.599 \\ 1413.1137 & 1413.423 & 1414.211 \\ 1414.8151 & 1415.899 & 1416.168 \\ 1420.6718 & 1421.019 & 1423.023 \\ 1423.9943 & 1426.869 & 1427.18 \\ 1429.6767 & 1429.888 & 1431.659 \\ 1432.4743 & 1475.394 & 1476.491 \\ 1501.2084 & 1501.434 & 1501.701 \\ 1502.0943 & 1502.475 & 1502.809 \\ 1502.9828 & 1503.181 & 1503.567 \\ 1503.8422 & 1509.704 & 1510.412 \\ 1510.6868 & 1510.761 & 1510.952 \\ 1511.0907 & 1511.554 & 1511.875 \\ 1511.9188 & 1512.631 & 1521.47 \\ 1521.5745 & 1521.61 & 1521.638 \\ 1521.7354 & 1521.752 & 1522.41 \\ 1522.4302 & 1523.095 & 1523.106 \\ 1532.3726 & 1532.429 & 1532.467 \\ 1532.5025 & 1532.787 & 1533.186 \\ 1533.7455 & 1534.28 & 1534.617 \\ 1534.691 & 1560.882 & 1567.435 \\ 1575.7914 & 1585.013 & 1594.097 \\ 1602.5201 & 1609.881 & 1611.83 \\ 1615.5531 & 1617.754 & 1621.429 \\ 1621.9978 & 1625.486 & 1633.503 \\ 1641.4593 & 1647.622 & 1652.259 \\ 1655.3519 & 1656.584 & 1685.634 \\ 1686.3915 & 3005.3 & 3006.737 \\ 3006.9911 & 3007.201 & 3007.338 \\ 3007.4433 & 3007.514 & 3007.582 \\ 3007.6683 & 3007.707 & 3007.936 \\ 3007.9918 & 3008.166 & 3008.206 \\ 3008.3461 & 3008.43 & 3008.946 \\ 3009.0123 & 3010.629 & 3010.833 \\ 3030.0126 & 3030.106 & 3030.127 \\ 3030.3546 & 3030.434 & 3030.511\end{array}$

$\begin{array}{rrr}3030.7972 & 3034.836 & 3034.845 \\ 3034.8702 & 3034.88 & 3034.997 \\ 3035.0061 & 3035.18 & 3035.197 \\ 3035.2446 & 3035.54 & 3057.375 \\ 3058.481 & 3058.625 & 3058.72 \\ 3058.8967 & 3058.924 & 3059.038 \\ 3059.1031 & 3059.259 & 3059.358 \\ 3059.4353 & 3059.463 & 3059.502 \\ 3059.5224 & 3059.705 & 3060.054 \\ 3060.1407 & 3061.376 & 3062.261 \\ 3062.3726 & 3078.127 & 3078.158 \\ 3078.1795 & 3078.255 & 3078.307 \\ 3078.3818 & 3078.451 & 3078.498 \\ 3078.587 & 3078.748 & 3082.345 \\ 3082.418 & 3082.505 & 3082.519 \\ 3082.5506 & 3082.59 & 3082.643 \\ 3082.6652 & 3082.786 & 3082.791 \\ 3175.7551 & 3175.993 & 3192.744 \\ 3195.8223 & 3213.05 & 3214.439 \\ 3217.6481 & 3218.349 & 3219.564 \\ 3221.6368 & 3222.592 & 3224.268 \\ 3234.6892 & 3238.439 & 3238.936 \\ 3241.4352 & 3242.617 & 3244.427 \\ 3245.8231 & 3248.779 & 3250.431 \\ 3251.8523 & 3254.987 & 3255.103\end{array}$

C (DP = 15)

$\begin{array}{rrr}2.5164 & 3.1877 & 4.0342 \\ 4.5955 & 6.097 & 7.6474 \\ 8.1007 & 8.132 & 9.1204 \\ 9.1597 & 10.058 & 10.3744 \\ 11.388 & 11.43 & 12.6257 \\ 17.3504 & 18.7407 & 19.1798 \\ 22.0226 & 24.5954 & 30.7592 \\ 36.7104 & 38.228 & 43.2921 \\ 45.9683 & 49.8306 & 49.8426 \\ 51.4081 & 51.4883 & 51.8456 \\ 52.1987 & 53.7571 & 54.3727 \\ 55.6658 & 55.8646 & 59.1115 \\ 61.3172 & 63.8113 & 66.9815 \\ 68.9085 & 70.2458 & 80.4162\end{array}$




\begin{tabular}{|c|c|c|}
\hline 81.1849 & 82.9676 & 85.6204 \\
\hline 91.6678 & 93.9578 & 95.3974 \\
\hline 99.2618 & 101.1401 & 104.2036 \\
\hline 108.1878 & 109.4309 & 110.4704 \\
\hline 110.5619 & 111.9374 & 112.2382 \\
\hline 112.8871 & 113.7924 & 114.8218 \\
\hline 119.9893 & 120.2954 & 127.4966 \\
\hline 145.4042 & 152.7731 & 153.1313 \\
\hline 155.8133 & 156.5752 & 156.8265 \\
\hline 156.8352 & 157.0547 & 158.1263 \\
\hline 158.3339 & 158.5799 & 161.3259 \\
\hline 162.0656 & 162.9471 & 164.111 \\
\hline 166.8725 & 168.1849 & 171.682 \\
\hline 173.9896 & 175.9502 & 176.4073 \\
\hline 177.709 & 178.9714 & 180.0207 \\
\hline 180.2304 & 180.8158 & 183.3184 \\
\hline 183.5754 & 189.9152 & 190.5742 \\
\hline 191.1725 & 198.0144 & 199.693 \\
\hline 203.1505 & 204.8358 & 206.2794 \\
\hline 206.6915 & 211.8376 & 213.4438 \\
\hline 234.6845 & 236.0272 & 240.6808 \\
\hline 244.4552 & 246.8099 & 250.6439 \\
\hline 253.1559 & 260.1668 & 277.2633 \\
\hline 290.6043 & 304.4743 & 308.8572 \\
\hline 309.0426 & 310.5074 & 311.0344 \\
\hline 311.2031 & 311.8353 & 312.1761 \\
\hline 312.872 & 312.8944 & 313.336 \\
\hline 314.3945 & 314.9321 & 315.1927 \\
\hline 315.9256 & 316.1674 & 329.9177 \\
\hline 345.3551 & 352.2481 & 354.6206 \\
\hline 360.5692 & 364.6408 & 365.1882 \\
\hline 380.7071 & 382.185 & 384.9865 \\
\hline 387.9679 & 390.6867 & 394.5186 \\
\hline 400.6699 & 408.4733 & 415.4647 \\
\hline 418.1349 & 419.7784 & 421.8259 \\
\hline 422.2013 & 424.3046 & 424.3573 \\
\hline 462.4249 & 462.8925 & 464.5138 \\
\hline 465.9298 & 468.2341 & 468.8289 \\
\hline 471.4666 & 473.0829 & 474.487 \\
\hline 477.4494 & 479.8965 & 481.7774 \\
\hline 483.3625 & 484.6258 & 488.2814 \\
\hline 488.406 & 491.8004 & 45 \\
\hline
\end{tabular}

\begin{tabular}{|c|c|c|}
\hline 493.1745 & 493.3254 & 493.6184 \\
\hline 493.8148 & 494.4262 & 494.78 \\
\hline 495.2228 & 496.5659 & 498.5324 \\
\hline 501.2973 & 504.3549 & 521.9286 \\
\hline 522.8208 & 524.7968 & 525.9814 \\
\hline 527.6694 & 530.7071 & 530.7777 \\
\hline 533.6009 & 534.2826 & 536.0571 \\
\hline 536.1686 & 539.0257 & 539.7755 \\
\hline 541.9724 & 542.4118 & 545.0755 \\
\hline 545.1799 & 552.0983 & 558.4391 \\
\hline 566.3898 & 576.2311 & 586.8512 \\
\hline 596.1746 & 604.7586 & 613.0201 \\
\hline 617.2964 & 618.4776 & 621.8852 \\
\hline 624.1114 & 625.9173 & 626.6262 \\
\hline 628.3401 & 629.9693 & 632.067 \\
\hline 633.8083 & 635.6495 & 637.8463 \\
\hline 638.8161 & 645.4009 & 653.9831 \\
\hline 663.6463 & 673.8193 & 683.9683 \\
\hline 693.3623 & 701.0672 & 707.0183 \\
\hline 715.1021 & 715.894 & 738.0407 \\
\hline 744.9812 & 752.3292 & 759.4377 \\
\hline 765.8461 & 771.1367 & 775.6197 \\
\hline 779.9019 & 783.4487 & 786.0218 \\
\hline 788.1061 & 789.709 & 790.7519 \\
\hline 791.2493 & 836.0652 & 836.1841 \\
\hline 836.6245 & 837.5212 & 837.5562 \\
\hline 837.6543 & 838.6788 & 838.9181 \\
\hline 839.5713 & 840.7948 & 841.9494 \\
\hline 843.3193 & 844.7274 & 845.8878 \\
\hline 856.7685 & 857.6527 & 859.367 \\
\hline 861.7392 & 864.6458 & 867.8439 \\
\hline 870.5162 & 872.8975 & 873.972 \\
\hline 874.4952 & 876.5444 & 877.2277 \\
\hline 879.2146 & 879.7916 & 882.2521 \\
\hline 882.3935 & 885.7054 & 886.3915 \\
\hline 887.8242 & 889.3269 & 890.0944 \\
\hline 890.2577 & 890.9021 & 893.8627 \\
\hline 896.79 & 899.3039 & 901.7887 \\
\hline 904.022 & 905.8861 & 907.8832 \\
\hline 908.556 & 908.6421 & 908.9763 \\
\hline 909.3642 & 909.5869 & 910.1633 \\
\hline 910.322 & 910.9872 & 911.5629 \\
\hline
\end{tabular}




$\begin{array}{rrr}912.3944 & 912.577 & 912.6087 \\ 912.9961 & 913.7183 & 914.1082 \\ 914.3503 & 914.4898 & 931.4408 \\ 931.9233 & 932.2678 & 932.8916 \\ 933.5114 & 934.0544 & 934.7735 \\ 935.4875 & 935.9994 & 936.5624 \\ 937.2543 & 937.7304 & 937.9583 \\ 938.3583 & 938.5327 & 959.5822 \\ 961.4751 & 965.7541 & 967.2319 \\ 968.366 & 973.6709 & 974.9208 \\ 975.4658 & 979.7115 & 980.9364 \\ 982.5671 & 982.9435 & 983.7295 \\ 984.2737 & 987.3207 & 987.321 \\ 995.4799 & 995.6613 & 996.5414 \\ 996.6355 & 997.7085 & 998.0827 \\ 998.7145 & 999.9389 & 1001.776 \\ 1003.34 & 1004.729 & 1005.602 \\ 1005.916 & 1020.005 & 1020.006 \\ 1047.343 & 1047.345 & 1077.91 \\ 1077.977 & 1078.481 & 1078.578 \\ 1078.877 & 1079.672 & 1080.907 \\ 1081.838 & 1082.735 & 1084.014 \\ 1086.066 & 1088.639 & 1091.225 \\ 1094.013 & 1097.197 & 1097.755 \\ 1173.798 .174 & 1098.266 & 1099.539 \\ 1100.441 & 1201.923 & 1203.41\end{array}$

\begin{tabular}{rrr}
1204.3 & 1204.554 & 1204.789 \\
1205.154 & 1205.191 & 1215.192 \\
1215.448 & 1215.68 & 1216.416 \\
1217.289 & 1218.358 & 1220.09 \\
1222.064 & 1224.009 & 1225.161 \\
1225.188 & 1225.68 & 1225.688 \\
1226.241 & 1226.304 & 1280.869 \\
1280.869 & 1282.688 & 1283.021 \\
1283.42 & 1284.234 & 1285.652 \\
1287.497 & 1289.625 & 1291.212 \\
1291.663 & 1291.819 & 1291.859 \\
1292.528 & 1292.738 & 1292.933 \\
1293.157 & 1293.186 & 1293.337 \\
1293.339 & 1293.675 & 1293.835 \\
1294.127 & 1294.459 & 1294.653 \\
1295.33 & 1297.58 & 1299.49 \\
1301.078 & 1301.833 & 1321.361 \\
1321.361 & 1323.288 & 1323.299 \\
1324.21 & 1324.228 & 1325.077 \\
1325.127 & 1325.501 & 1325.549 \\
1325.748 & 1326.146 & 1326.575 \\
1326.987 & 1327.187 & 1332.131 \\
1332.333 & 1334.055 & 1334.59 \\
1335.531 & 1336.319 & 1337.714 \\
1338.248 & 1339.432 & 1344.169 \\
1349.11 & 1353.54 & 1357.565 \\
1357.584 & 1361.263 & 1361.52 \\
1363.936 & 1366.336 & 1370.982 \\
1375.577 & 1379.669 & 1383.72 \\
1387.205 & 1388.978 & 1389.797 \\
1389.973 & 1390.287 & 1390.389 \\
1390.489 & 1390.578 & 1390.685 \\
1390.797 & 1390.945 & 1391.158 \\
1391.305 & 1391.348 & 1391.375 \\
1391.451 & 1391.704 & 1391.754 \\
1391.771 & 1392.347 & 1393.661 \\
1394.903 & 1395.258 & 1395.492 \\
\hline 1409.097 & 1395.845 & 1396.066 \\
13974 & 1396.673 & 1397.825 \\
\hline 1401.842 & 1403.615 \\
\hline 1407.907
\end{tabular}




\begin{tabular}{|c|c|c|}
\hline 116 & 1412.146 & 1412.198 \\
\hline 12.428 & 1412.76 & 1413.103 \\
\hline 1413.429 & 1413.764 & 1414.323 \\
\hline 1414.816 & 1415.279 & 1415.981 \\
\hline 1416.248 & 1416.881 & 1420.22 \\
\hline 1420.963 & 1421.056 & 1422.781 \\
\hline 1423.407 & 1425.545 & 1426.137 \\
\hline 1428.253 & 1428.402 & 1430.309 \\
\hline 1430.438 & 1431.592 & 1432.074 \\
\hline 1432.699 & 1433.127 & 1475.054 \\
\hline 1475.054 & 1501.056 & 1501.077 \\
\hline 1501.238 & 1501.661 & 1501.934 \\
\hline 1501.959 & 1502.099 & 1502.527 \\
\hline 1502.696 & 1502.73 & 1503.052 \\
\hline 1503.51 & 1503.518 & 1503.783 \\
\hline 1503.852 & 1509.432 & 1509.442 \\
\hline 1509.948 & 1510.279 & 1510.286 \\
\hline 1510.706 & 1510.896 & 1510.905 \\
\hline 1510.962 & 1511.292 & 1511.367 \\
\hline 1511.517 & 1511.518 & 1512.535 \\
\hline 1512.536 & 1521.551 & 1521.559 \\
\hline 1521.577 & 1521.598 & 1521.614 \\
\hline 1521.911 & 1521.912 & 1522.355 \\
\hline 1522.355 & 1522.463 & 1522.463 \\
\hline 1522.538 & 1522.539 & 1522.589 \\
\hline 1522.59 & 1532.448 & 1532.484 \\
\hline 1532.502 & 1532.749 & 1532.756 \\
\hline 1533.076 & 1533.077 & 1533.138 \\
\hline 1533.139 & 1533.238 & 1533.238 \\
\hline 1533.426 & 1533.427 & 1533.446 \\
\hline 1533.447 & 1559.159 & 1562.232 \\
\hline 1566.627 & 1571.866 & 1577.683 \\
\hline 1584.058 & 1590.46 & 1596.205 \\
\hline 1601.69 & 1606.773 & 1610.922 \\
\hline 1611.19 & 1614.254 & 1614.785 \\
\hline 1617.207 & 1618.152 & 1621.547 \\
\hline 1621.677 & 1622.992 & 1628.482 \\
\hline 1633.796 & 1638.259 & 1642.669 \\
\hline 1647.088 & 1650.746 & 1653.338 \\
\hline 1655.115 & 1656.837 & 1657.048 \\
\hline 1685.38 & 1685.382 & 3005.355 \\
\hline 3005.387 & 3006.504 & 6.52 \\
\hline
\end{tabular}

$\begin{array}{rrr}3006.675 & 3006.695 & 3007.229 \\ 3007.269 & 3007.284 & 3007.304 \\ 3007.33 & 3007.371 & 3007.392 \\ 3007.467 & 3007.472 & 3007.579 \\ 3007.643 & 3007.824 & 3007.824 \\ 3007.889 & 3007.895 & 3008.039 \\ 3008.331 & 3008.348 & 3008.593 \\ 3008.593 & 3009.578 & 3009.582 \\ 3010.528 & 3010.532 & 3029.963 \\ 3029.964 & 3030.057 & 3030.065 \\ 3030.068 & 3030.07 & 3030.123 \\ 3030.153 & 3030.169 & 3030.171 \\ 3030.218 & 3030.325 & 3030.37 \\ 3030.42 & 3030.438 & 3034.773 \\ 3034.774 & 3034.798 & 3034.813 \\ 3034.823 & 3034.855 & 3034.857 \\ 3034.862 & 3034.863 & 3034.977 \\ 3034.979 & 3035.06 & 3035.062 \\ 3035.245 & 3035.246 & 3057.942 \\ 3057.969 & 3058.058 & 3058.067 \\ 3058.097 & 3058.107 & 3058.533 \\ 3058.543 & 3058.844 & 3059.041 \\ 3059.091 & 3059.231 & 3059.301 \\ 3059.382 & 3059.537 & 3059.555 \\ 3059.812 & 3059.831 & 3059.994 \\ 3060.012 & 3060.302 & 3060.304 \\ 3060.414 & 3060.427 & 3060.826 \\ 3060.827 & 3061.609 & 3061.621 \\ 3062.079 & 3062.093 & 3077.786 \\ 3077.79 & 3077.942 & 3077.955 \\ 3078.101 & 3078.105 & 3078.146 \\ 3078.179 & 3078.284 & 3078.338 \\ 3078.36 & 3078.4 & 3078.488 \\ 3078.568 & 3078.634 & 3082.208 \\ 3082.212 & 3082.325 & 3082.328 \\ 3082.354 & 3082.432 & 3082.482 \\ 3082.498 & 3082.499 & 3082.559 \\ 3082.564 & 3082.648 & 3082.648 \\ 3082.767 & 3082.769 & 3174.772 \\ 3174.773 & 3194.175 & 3194.178 \\ 3215.801 & 3217.673 \\ 32.036 & 3219.483\end{array}$


$\begin{array}{lll}3220.349 & 3221.086 & 3221.259\end{array}$

$3222.016 \quad 3222.658 \quad 3223.694$

$3224.877 \quad 3237.945 \quad 3237.948$

$3239.823 \quad 3239.867 \quad 3241.775$
$3243.217 \quad 3244.976 \quad 3245.789$

$3246.202 \quad 3248.202 \quad 3248.941$

$3249.192 \quad 3250.318 \quad 3251.625$

$\begin{array}{lll}3252.417 & 3253.694 & 3253.695\end{array}$ 